Prepared for the U.S. Department of Energy

Under Contract DE-AC05-76RL01830

\title{
Evaluation of Promoters for Rhodium-Based Catalysts for Mixed Alcohol Synthesis
}
MA Gerber
M Gray
JF White
DJ Stevens

December 2008

Pacific Northwest

NATIONAL LABORATORY 


\title{
DISCLAIMER
}

This report was prepared as an account of work sponsored by an agency of the United States Government. Neither the United States Government nor any agency thereof, nor Battelle Memorial Institute, nor any of their employees, makes any warranty, express or implied, or assumes any legal liability or responsibility for the accuracy, completeness, or usefulness of any information, apparatus, product, or process disclosed, or represents that its use would not infringe privately owned rights. Reference herein to any specific commercial product, process, or service by trade name, trademark, manufacturer, or otherwise does not necessarily constitute or imply its endorsement, recommendation, or favoring by the United States Government or any agency thereof, or Battelle Memorial Institute. The views and opinions of authors expressed herein do not necessarily state or reflect those of the United States Government or any agency thereof.

\author{
PACIFIC NORTHWEST NATIONAL LABORATORY \\ operated by \\ BATTELLE \\ for the \\ UNITED STATES DEPARTMENT OF ENERGY \\ under Contract DE-AC05-76RL01830 \\ Printed in the United States of America \\ Available to DOE and DOE contractors from the \\ Office of Scientific and Technical Information, \\ P.O. Box 62, Oak Ridge, TN 37831-0062; \\ ph: (865) 576-8401 \\ fax: (865) 576-5728 \\ email: reports@adonis.osti.gov

\footnotetext{
Available to the public from the National Technical Information Service, U.S. Department of Commerce, 5285 Port Royal Rd., Springfield, VA 22161 ph: (800) 553-6847 fax: $(703) 605-6900$ email: orders@ntis.fedworld.gov

online ordering: http://www.ntis.gov/ordering.htm
} 


\title{
Evaluation of Promoters for Rhodium-Based Catalysts for Mixed Alcohol Synthesis
}

\author{
MA Gerber \\ M Gray \\ JF White \\ DJ Stevens
}

December 2008

Prepared for

the U.S. Department of Energy

under Contract DE-AC05-76RL01830

Pacific Northwest National Laboratory

Richland, Washington 99352 



\section{Executive Summary}

The U.S. Department of Energy's (DOE) Pacific Northwest National Laboratory (PNNL) and National Renewable Energy Laboratory (NREL) are conducting research to investigate the feasibility of producing mixed alcohols from biomass-derived synthesis gas (syngas). PNNL is tasked with obtaining commercially-available catalysts or preparing promising mixed alcohol catalysts and screening them in a laboratory-scale reactor system. Commercially-available catalysts and the most promising experimental catalysts are provided to NREL for testing using a slipstream from a pilot-scale biomass gasifier.

After a review of the literature in 2006 and conversations at that time with companies that produce catalysts, we concluded that commercial, mixed alcohol synthesis catalysts were not available. One catalyst manufacturer did supply a modified methanol catalyst (MeOH-X) that was tested in the PNNL laboratory-scale system and then provided to NREL for further testing. PNNL also prepared and tested the behavior of 10 other catalysts that represented the distinct catalyst classes for mixed alcohol syntheses. The catalyst with the best combination of $\mathrm{C}_{2}+$ oxygenates space time yield (STY), and selectivity was a silica-supported catalyst containing rhodium (Rh) and manganese (Mn). Based on these results, subsequent testing in 2007 and 2008 focused on the performance of the Rh-based catalyst to determine the effects of adding promoters to the Rh catalysts in addition to the Mn promoter already being used.

A total of 28 tests were conducted to evaluate 22 different promoters as well as the unpromoted catalyst. The test conditions and the range of $\mathrm{C}_{2}+$-oxygenate $\mathrm{STYs}$ for these catalysts and the previously tested Rh-based catalysts are shown in Table S.1. Some of the earlier tests were conducted using a clamshell furnace to heat the reactor. However, we found that temperature control of the catalyst was difficult if not impossible to maintain for the more active catalysts, particularly when the carbon conversion was above approximately $30 \%$. Catalysts tested with the clamshell furnace are identified in Table S.1. The clamshell furnace was replaced with a hot oil circulating system, which gave much better control for the more active catalysts, although control was still difficult for some of the more active catalysts promoted by iridium (Ir), platinum (Pt), tin (Sn), gold ( $\mathrm{Au}$ ), and molybdenum (Mo). All of these catalysts also produced significant quantities of hydrocarbon liquids, which may be related to the difficulty with temperature control (other catalysts that produced significant quantities of hydrocarbon liquids were heated with the furnace). In tests conducted with the Ir-promoted catalyst using both the furnace and the oil heating system, $\mathrm{C}_{2}+$-oxygenate STYs were higher when the oil-heating system was used. This finding suggests that similar improvements in the STYs may be possible for the catalysts that were only tested with the furnace.

The source of the silica $\left(\mathrm{SiO}_{2}\right)$ was another point of interest during testing. Although all of the tests were performed with Davisil $645 \mathrm{SiO}_{2}, \mathrm{SiO}_{2}$ from different sources was used to prepare the catalysts. Most of the catalysts tested initially used an earlier lot of the Davisil $\mathrm{SiO}_{2}$ obtained from Altech Inc., (i.e., the catalysts promoted by iron [Fe], lithium [Li], Ir, nickel [Ni], rhenium [Re], copper [Cu], cobalt [Co], and Mo). Subsequent catalysts were prepared from more recent $\mathrm{SiO}_{2}$ lots obtained from Fisher Scientific. The catalysts prepared using the $\mathrm{SiO}_{2}$ obtained from Fisher Scientific had a tendency to produce significant quantities of methanol while those prepared using the $\mathrm{SiO}_{2}$ from Altech did not. There were exceptions for both, but in tests conducted using the unpromoted $\mathrm{RhMn} / \mathrm{SiO}$ catalyst prepared from both sources of $\mathrm{SiO}_{2}$, the sample prepared with the Fisher Scientific $\mathrm{SiO}_{2}$ had lower $\mathrm{C}_{2}+-$ oxygenate STYs than those made using the Altech $\mathrm{SiO}_{2}$. The sample made with the Fisher Scientific $\mathrm{SiO}_{2}$ also produced significant amounts of methanol. Further research is needed to explain this behavior. 
Table S.1. Test Conditions

\begin{tabular}{|c|c|c|c|c|c|c|c|}
\hline Catalyst & Promoters & $\begin{array}{c}\text { Pressure } \\
\text { (atm) }\end{array}$ & $\begin{array}{c}\text { Temperature } \\
\left({ }^{\circ} \mathrm{C}\right) \\
\end{array}$ & $\begin{array}{c}\text { GHSV } \\
\left(\mathrm{L} / \mathrm{L}_{\mathrm{cat}} / \mathrm{hr}\right)\end{array}$ & $\begin{array}{c}\text { STY of } \mathrm{C}_{2}^{+} \\
\text {Oxygenates }^{(\mathrm{a})} \\
\left(\mathrm{g} / \mathrm{L}_{\mathrm{cat}} / \mathrm{hr}\right) \\
\end{array}$ & $\begin{array}{c}\text { Selectivity } \\
\text { to } \mathrm{C}_{2}+ \\
\text { Oxygenates } \\
(\%) \\
\end{array}$ & $\begin{array}{c}\text { Ratio of } \\
\mathrm{C}_{2}+ \\
\text { Alcohols to } \\
\mathrm{C}_{2}+ \\
\text { Oxygenates } \\
\end{array}$ \\
\hline $\mathrm{Rh} / \mathrm{Mn} / \mathrm{SiO}_{2}{ }^{(\mathrm{b})}$ & $\mathrm{Mn}$ & 80 & $255-324$ & $3,300-15,000$ & $20-440$ & $23-60$ & $0.26-0.51$ \\
\hline $\mathrm{Rh} / \mathrm{Mn} / \mathrm{Ir} / \mathrm{SiO}_{2}{ }^{(\mathrm{b})}$ & $\mathrm{Mn}, \mathrm{Ir}$ & 80 & $256-328$ & $7,500-15,000$ & $290-810$ & $23-58$ & $0.10-0.37$ \\
\hline $\mathrm{RhMnPt} / \mathrm{SiO}_{2}$ & $\mathrm{Mn}, \mathrm{Pt}$ & 80 & $255-315$ & $7,500-15,000$ & $200-660$ & $38-51$ & $0.11-0.17$ \\
\hline $\mathrm{Rh} / \mathrm{Mn} / \mathrm{Au} / \mathrm{SiO}_{2}$ & $\mathrm{Mn}, \mathrm{Au}$ & 80 & $256-325$ & $7,500-19,000$ & $110-500$ & $28-55$ & $0.15-0.44$ \\
\hline $\mathrm{Rh} / \mathrm{Mn} / \mathrm{Li} / \mathrm{SiO}_{2}{ }^{(\mathrm{c})}$ & $\mathrm{Mn}, \mathrm{Li}$ & 80 & $256-350$ & $7,500-11,000$ & $90-480$ & $34-62$ & $0.19-0.56$ \\
\hline $\mathrm{Rh} / \mathrm{Mn} / \mathrm{Ni} / \mathrm{SiO}_{2}{ }^{(\mathrm{c})}$ & $\mathrm{Mn}, \mathrm{Ni}$ & 80 & $256-325$ & $7,500-11,000$ & $130-480$ & $21-46$ & $0.17-0.36$ \\
\hline $\mathrm{Rh} / \mathrm{Mn} / \mathrm{Mo} / \mathrm{SiO}_{2}$ & Mn, Mo & 80 & $256-325$ & $7,500-15,000$ & $160-470$ & $12-43$ & $0.19-0.43$ \\
\hline $\mathrm{Rh} / \mathrm{MnRu} / \mathrm{SiO}_{2}$ & $\mathrm{Mn}, \mathrm{Ru}$ & 80 & $256-325$ & $7,500-15,000$ & $200-440$ & $20-36$ & $0.10-0.32$ \\
\hline $\mathrm{Rh} / \mathrm{Mn} / \mathrm{Fe} / \mathrm{SiO}_{2}{ }^{(\mathrm{c})}$ & $\mathrm{Mn}, \mathrm{Fe}$ & 80 & $257-402$ & $7,500-15,000$ & $170-400$ & $11-42$ & $0.32-0.75$ \\
\hline $\mathrm{Rh} / \mathrm{Mn} / \mathrm{Sn} / \mathrm{SiO}_{2}$ & $\mathrm{Mn}, \mathrm{Sn}$ & 80 & $256-342$ & $7,500-15,000$ & $50-350$ & $35-59$ & $0.39-0.57$ \\
\hline $\mathrm{Rh} / \mathrm{Mn} / \mathrm{Co} / \mathrm{SiO}_{2}$ & $\mathrm{Mn}, \mathrm{Co}$ & 80 & $256-323$ & $7,500-11,000$ & $140-350$ & $21-50$ & $0.19-0.46$ \\
\hline $\mathrm{Rh} / \mathrm{Mn} / \mathrm{Pd} / \mathrm{SiO}_{2}$ & $\mathrm{Mn}, \mathrm{Pd}$ & 80 & $256-323$ & $7,500-11,000$ & $100-300$ & $19-50$ & $0.14-0.38$ \\
\hline $\mathrm{Rh} / \mathrm{Mn} / \mathrm{V} / \mathrm{SiO}_{2}$ & $\mathrm{Mn}, \mathrm{V}$ & 80 & $255-315$ & $7,500-15,000$ & $100-370$ & $23-44$ & $0.18-0.47$ \\
\hline $\mathrm{Rh} / \mathrm{Mn} / \mathrm{Re} / \mathrm{SiO}_{2}{ }^{(\mathrm{c})}$ & $\mathrm{Mn}, \mathrm{Re}$ & 80 & $256-324$ & $7,500-11,000$ & $210-260$ & $16-36$ & $0.25-0.61$ \\
\hline $\mathrm{Rh} / \mathrm{Mn} / \mathrm{Ga} / \mathrm{SiO}_{2}$ & $\mathrm{Mn}, \mathrm{Ga}$ & 80 & $256-345$ & $7,500-11,000$ & $10-190$ & $26-46$ & $0.64-0.69$ \\
\hline $\mathrm{Rh} / \mathrm{Mn} / \mathrm{Cu} / \mathrm{SiO}_{2}$ & $\mathrm{Mn}, \mathrm{Cu}$ & 80 & $256-337$ & $7,500-11,000$ & $60-150$ & $21-46$ & $0.34-0.62$ \\
\hline $\mathrm{Rh} / \mathrm{Mn} / \mathrm{In} / \mathrm{SiO}_{2}$ & Mn, In & 80 & $256-345$ & $7,500-11,000$ & $20-140$ & $33-66$ & $0.56-0.93$ \\
\hline $\mathrm{Rh} / \mathrm{MnCs} / \mathrm{SiO}_{2}$ & $\mathrm{Mn}, \mathrm{Cs}$ & 80 & $256-325$ & $7,500-15,000$ & $20-120$ & $24-40$ & $0.10-0.24$ \\
\hline $\mathrm{Rh} / \mathrm{Mn} / \mathrm{Ag} / \mathrm{SiO}_{2}$ & $\mathrm{Mn}, \mathrm{Ag}$ & 80 & $256-325$ & $7,500-15,000$ & $30-110$ & $21-51$ & $0.58-0.68$ \\
\hline $\mathrm{Rh} / \mathrm{Mn} / \mathrm{Pb} / \mathrm{SiO}_{2}$ & $\mathrm{Mn}, \mathrm{Pb}$ & 80 & $257-325$ & $7,500-11,000$ & $0-10$ & NA-18 & NA-0.45 \\
\hline $\mathrm{Rh} / \mathrm{Mn} / \mathrm{Ge} / \mathrm{SiO}_{2}$ & $\mathrm{Mn}, \mathrm{Ge}$ & 80 & $256-326$ & $7,500-11,000$ & $0-20$ & $5-21$ & $0.092-0.96$ \\
\hline $\mathrm{Rh} / \mathrm{Mn} / \mathrm{Bi} / \mathrm{SiO}_{2}$ & $\mathrm{Mn}, \mathrm{Bi}$ & 80 & $257-345$ & $7,500-11,000$ & $0-10$ & NA-20 & NA-0.7 \\
\hline $\mathrm{Rh} / \mathrm{MnTe} / \mathrm{SiO}_{2}$ & $\mathrm{Mn}, \mathrm{Te}$ & 80 & $256-326$ & $7,500-11,000$ & 0 & NA & NA \\
\hline
\end{tabular}

(a) $\mathrm{C}_{2}+$ oxygenates were predominantly $\mathrm{C}_{2}$ to $\mathrm{C}_{5}$ alcohols, acetic acid, acetaldehyde, and ethyl acetate.

(b) Catalyst tested using both furnace and oil heating system to heat reactor.

(c) Catalyst only tested using furnace to heat reactor.

Note: Rh:Mn:M ( $\mathrm{M}=$ promoter $)$ atomic ratios were 1.00:0.57:0.10, except for Li promotion, which was 1.00:0.57:0.30.

Taking into consideration these caveats in testing, the following general trends were observed for the test results:

- The highest carbon selectivity to $\mathrm{C}_{2}+$ oxygenates occurred at the lowest reaction temperatures and accompanying lowest STYs.

- The lowest carbon selectivity to $\mathrm{C}_{2}+$ oxygenates occurred at the highest reaction temperatures because of high carbon conversion to hydrocarbons. 
- The highest $\mathrm{C}_{2}+$-oxygenate STYs occurred between $300^{\circ} \mathrm{C}$ and $325^{\circ} \mathrm{C}$, with the gas hourly space velocity adjusted when necessary to maintain carbon conversion ranges between approximately 30 and $40 \%$. Higher carbon selectivity to hydrocarbons at higher temperatures resulted in lower $\mathrm{C}_{2}+-$ oxygenate STYs.

- When catalysts were heated to between 300 and $325^{\circ} \mathrm{C}$ the catalysts showed evidence of some deactivation with respect to $\mathrm{C}_{2}+$ oxygenate productivity, accompanied by reduced chain growth for the hydrocarbon products. The degree of deactivation and the temperature at which it occurred varied between the different catalysts tested.

Table S.1 ranks the catalysts in decreasing order with respect to the maximum $\mathrm{C}_{2}+$-oxygenate STYs achieved during testing (the $\mathrm{RhMn} / \mathrm{SiO}_{2}$ catalyst is at the top of the list for comparison). The catalyst promoted with Ir stood out in terms of significantly improving the STY of oxygenates with an observed maximum STY of approximately $810 \mathrm{~g} / \mathrm{L}_{\text {cat }} / \mathrm{hr}$ followed by Pt with observed maximum STY of 660 $\mathrm{g} / \mathrm{L}_{\text {cat }} / \mathrm{hr}$. The catalysts promoted by $\mathrm{Au}, \mathrm{Li}, \mathrm{Ni}, \mathrm{Mo}$, and $\mathrm{Ru}$ also had observed maximum STYs that were higher than that achieved for the unpromoted $\mathrm{RhMn} / \mathrm{SiO}_{2}$ catalyst, although the $\mathrm{Ru}$ - and Mo-promoted catalysts rapidly deactivated during testing and are considered unsuitable.

Of all of the catalysts evaluated, the Li-promoted catalysts had the highest carbon selectivity to $\mathrm{C}_{2}+$ oxygenates (47\%) under the conditions at which the maximum $\mathrm{C}_{2}+$-oxygenate STYs were obtained. The catalysts promoted with $\mathrm{Ir}, \mathrm{Pt}$, and $\mathrm{Au}$ had carbon selectivities to $\mathrm{C}_{2}+$ oxygenates at maximum $\mathrm{C}_{2}+$-oxygenate STYs, of 39, 39, and 37\%, respectively, which were better than the unpromoted catalyst (carbon selectivity of 32\%). None of the catalysts with higher STYs than the baseline catalyst had high carbon selectivities to $\mathrm{C}_{2}+$ alcohols relative to the total oxygenates. Only the gallium (Ga)- and indium (In)-promoted catalysts had very high ratios of $\mathrm{C}_{2}+$ alcohols to total $\mathrm{C}_{2}+$ oxygenates ratios, with ratios of 0.67 and 0.71 , respectively. These same catalysts had total alcohol:oxygenates ratios of 0.87 and 0.89 , respectively. 



\section{Acronyms and Abbreviations}

\begin{tabular}{|c|c|}
\hline $\mathrm{Au}$ & gold \\
\hline $\mathrm{CO}$ & carbon monoxide \\
\hline Co & cobalt \\
\hline $\mathrm{CO}_{2}$ & carbon dioxide \\
\hline Cs & cesium \\
\hline $\mathrm{Cu}$ & copper \\
\hline DOE & U.S. Department of Energy \\
\hline $\mathrm{Fe}$ & iron \\
\hline FT & Fischer-Tropsch \\
\hline $\mathrm{Ga}$ & gallium \\
\hline GC & gas chromatograph \\
\hline GHSV & gas hourly space velocity \\
\hline $\mathrm{H}_{2}$ & hydrogen \\
\hline HPLC & high-pressure liquid chromatograph \\
\hline In & indium \\
\hline $\operatorname{Ir}$ & iridium \\
\hline $\mathrm{Li}$ & lithium \\
\hline $\mathrm{MeOH}-\mathrm{X}$ & modified methanol catalyst \\
\hline $\mathrm{Mn}$ & manganese \\
\hline Mo & molybdenum \\
\hline $\mathrm{N}_{2}$ & nitrogen \\
\hline $\mathrm{Ni}$ & nickel \\
\hline NREL & National Renewable Energy Laboratory \\
\hline $\mathrm{Pt}$ & platinum \\
\hline PNNL & Pacific Northwest National Laboratory \\
\hline
\end{tabular}




$\begin{array}{ll}\mathrm{Re} & \begin{array}{l}\text { rhenium } \\ \text { rhodium }\end{array} \\ \mathrm{Sh} & \text { sulfur } \\ \mathrm{SiO}_{2} & \text { silica } \\ \mathrm{Sn} & \text { tin } \\ \mathrm{STY} & \text { space-time yield } \\ \text { syngas } & \text { synthesis gas } \\ \mathrm{V} & \\ \mathrm{Zn} & \text { vanadium } \\ \end{array}$




\section{Contents}

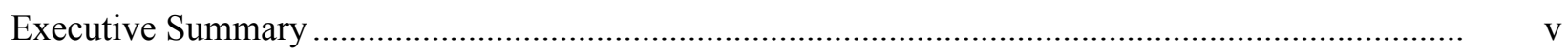

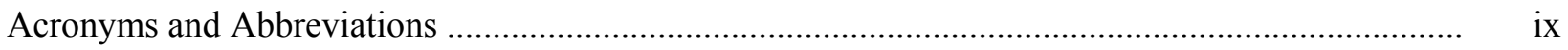

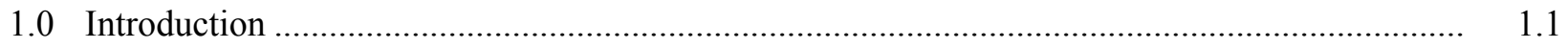

1.1 Catalyst Performance Requirements .................................................................... 1.1

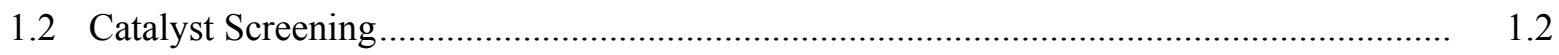

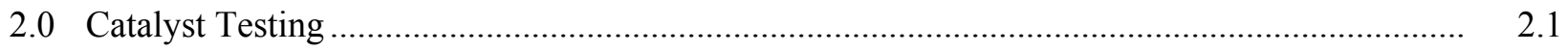

2.1 Synthesis Reactor System Description.................................................................... 2.1

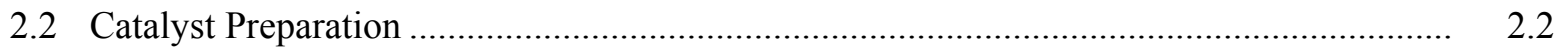

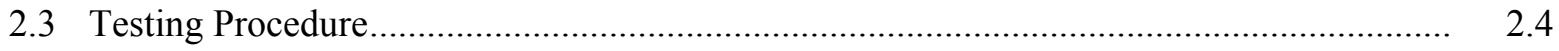

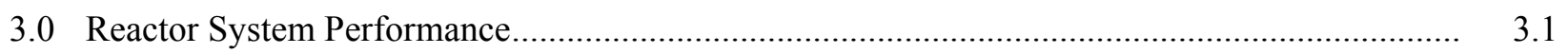

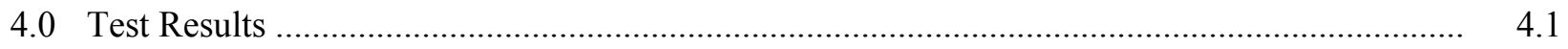

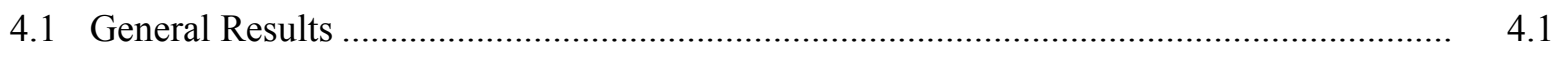

4.2 Comparison of Catalyst Performance ....................................................................... 4.2

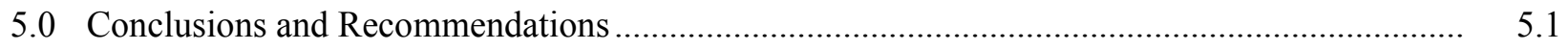

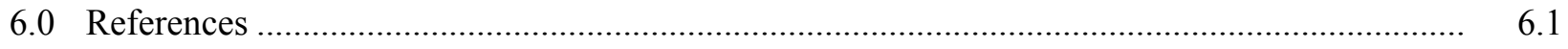

Appendix A - Catalyst Testing Data _............................................................................... A. A

Appendix B - Summary of Individual Test Results ..................................................................... B.1

\section{Figures}

2.1 Simplified Diagram of Reactor System ................................................................... 2.2

4.1 STYs of Promoted Catalysts at Conditions for Maximum STYs ............................................ 4.5

4.2 Carbon Selectivities of Promoted Catalysts at Conditions for Maximum STYs ........................ 4.7

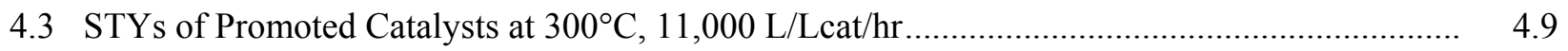

4.4 Carbon Selectivities of Promoted Catalysts at $300^{\circ} \mathrm{C}, 11,000 \mathrm{~L} / \mathrm{L}_{\mathrm{cat}} / \mathrm{hr}$................................. 4.11

\section{Tables}

2.1 List of Catalysts Tested and Corresponding Labels.

4.1 Comparison of Carbon Conversions and STYs of Promoted $\mathrm{RhMn} / \mathrm{SiO}_{2}$ Catalysts at Conditions Maximizing $\mathrm{C}_{2}+-$ Oxygenate STYs.

4.2 Comparison of Carbon Selectivities to Products of Promoted $\mathrm{RhMn} / \mathrm{SiO}_{2}$ Catalysts at Conditions Maximizing $\mathrm{C}_{2}+-$ Oxygenate STYs.

4.3 Comparison of Carbon Conversions and STYs of Promoted $\mathrm{RhMn} / \mathrm{SiO}_{2}$ Catalysts at $300^{\circ} \mathrm{C}$, $11,000 \mathrm{~L} / \mathrm{L}_{\text {cat }} / \mathrm{hr}$.....

4.4 Comparison of Carbon Selectivities to Products of Promoted $\mathrm{RhMn} / \mathrm{SiO}_{2}$ Catalysts at $300^{\circ} \mathrm{C}$, $11000 \mathrm{~L} / \mathrm{L}_{\text {cat }} / \mathrm{hr}$ 



\subsection{Introduction}

The U.S. Department of Energy's (DOE) National Renewable Energy Laboratory (NREL) conducted technical and economic assessments of the feasibility of producing mixed alcohols as a primary product from biomass-derived synthesis gas (syngas) to complement alcohol fuel biosynthesis in an integrated biorefinery (Phillips et al. 2007). The gasification process was based on a low-pressure, indirectly heated, entrained flow gasifier developed by Battelle. Downstream gas conditioning steps such as tar and light hydrocarbon reforming, sulfur (S) removal, and gas compression were included to produce a syngas suitable for mixed alcohol fuel synthesis. After a review of the literature, the mixed alcohol synthesis process was based on the expected performance of a cobalt (Co)/molybdenum (Mo) sulfide catalyst using methanol recycle in the synthesis reactor. The process increased the yield of higher alcohols and potentially recovered and recycled carbon dioxide $\left(\mathrm{CO}_{2}\right)$ from the product gas stream to a steam reformer to facilitate obtaining the desired syngas composition. Ethanol and higher alcohols were identified as the major products. Methane, light hydrocarbons, unreacted carbon monoxide $(\mathrm{CO})$, and hydrogen $\left(\mathrm{H}_{2}\right)$ were recycled to the synthesis reactor to maximize $\mathrm{CO}$ conversion. A purge stream taken from the recycled gas loop prevented excessive buildup of certain compounds.

\subsection{Catalyst Performance Requirements}

The assumptions regarding the performance of the mixed alcohol fuel synthesis catalyst were integral parts of the techno-economic assessment of this process. The performance of the catalyst affects the capital costs of the synthesis process in terms of reactor vessel size for a given throughput and gas recycle requirements, as well as vessel wall materials and thickness to meet design requirements for the expected operating pressure and temperature. Catalyst performance also affects the operating costs of the process in terms of energy required to pressurize and heat the incoming gas feed and the yield and distribution of key products (higher alcohols) and potential byproducts with significant economic value.

The mixed alcohol synthesis catalyst forming the basis of the evaluation was based on a class of catalysts consisting of alkali-doped Mo sulfide with other metals such as Co added to promote the selective production of mixed alcohols. One purported advantage of this class of catalyst is its tolerance for $\mathrm{S}$ in the syngas. Operating conditions and catalyst performance were based in part on 1) the catalysts developed and tested by Quarderer (1986) and Quarderer and Cochran (1986) and patented by Dow Chemical Company and 2) on typical values reported in the literature for similar catalysts in this class.

After review of the literature and conversations with selected catalyst manufacturers, we determined that no Mo-based catalysts were commercially available at that time. In fact, the only mixed alcohol synthesis catalyst offered by a catalyst manufacturer at the time of our initial investigation was a modified methanol catalyst (MeOH-X). The only other company found to have previously offered a commercial catalyst was the Institut Francais du Petrole in France, which developed catalysts based on copper $(\mathrm{Cu}) / \mathrm{Co}$ and $\mathrm{Cu} /$ nickel $(\mathrm{Ni})$ systems. Their work has been discontinued, and there is no longer any catalyst available for testing.

While testing was conducted using the modified methanol catalyst, a review of prior research was underaken to identify other potential catalysts, including Mo-based catalysts that showed promise for mixed alcohol synthesis. The primary screening and selection criterion for catalyst performance was the space-time yield (STY) of $\mathrm{C}_{2}+$-oxygenated hydrocarbons, with consideration given to the coproduction of 
methanol and liquid hydrocarbons. While the NREL techno-economic study cited an STY of 250 to $350 \mathrm{~g}$ mixed alcohol $/ \mathrm{L}_{\mathrm{cat}} / \mathrm{hr}$ as a productivity rate typical of the Mo catalysts, this value was considered marginal based on the assessment made by Stiles et al. (1991), who found that the STYs of catalysts used in commercial methanol synthesis plants ranged from approximately 670 to $1,340 \mathrm{~g}_{\mathrm{MeOH}} / \mathrm{L}_{\mathrm{cat}} / \mathrm{hr}$. According to Stiles and his co-workers, higher methanol production rates create heat dissipation requirements that are difficult to manage. Production rates involving higher alcohol production create higher exothermic heat loads than a comparable production rate of methanol. Furthermore, when significant methane or methane and higher hydrocarbons are produced along with the alcohols, heat dissipation can become unmanageable at the higher production rates. Consequently, it may not be practical to obtain higher alcohol production rates at the upper end of the range for methanol production in conventional fixed-bed reactor systems. On the other hand, rates significantly lower than the lower production range for methanol will likely result in unacceptable process economics.

\subsection{Catalyst Screening}

In the absence of commercial catalysts having higher alcohol production rates at levels needed to achieve economic viability, this project was undertaken to identify the most promising catalysts and the ones that had the best performance by testing them in a bench-scale system. Potential catalysts were divided into six general classes:

- Modified methanol catalysts (Cu/zinc [Zn] and $\mathrm{Cu} /$ manganese $[\mathrm{Mn}]$ based)

- Modified Mo sulfide catalysts

- Modified Mo oxide catalysts

- Rhodium (Rh)-based catalysts

- Modified FT catalysts.

The initial approach taken in this study was to obtain or prepare catalysts that were either representative of each class of catalysts or that had the potential to achieve high STYs for $\mathrm{C}_{2}+$ oxygenates, and to test them under conditions that would optimize $\mathrm{C}_{2}+$ STYs at a common operating pressure $(80 \mathrm{~atm})$. Ten catalysts representative of the different catalyst classes were prepared and tested along with a modified methanol catalyst provided by a catalyst manufacturer in 2006 and early 2007 (Gerber et al. 2007). Of these catalysts, only the modified Fischer-Tropsch (FT) and rhodium-based catalysts showed promise for achieving the necessary STYs. The two FT catalysts, which were modified to improve oxygenate yields, achieved $\mathrm{C}_{2}+$-oxygenate $\mathrm{STYs}$ that were within the recommended range. However, because of their much higher selectivity to FT liquids, the STYs for total organic liquids exceeded the recommended range under optimum operating conditions. Under test conditions that produced a total organic liquid within the recommended range (i.e., $1200 \mathrm{~g} / \mathrm{L}_{\text {cat }} / \mathrm{hr}$ total organic liquids STY $)$, one of these catalysts achieved a much lower $\mathrm{C}_{2}+$-oxygenate STY $\left(230 \mathrm{~g} / \mathrm{L}_{\text {cat }} / \mathrm{hr}\right)$ that, while higher than that achieved by the modified methanol and modified molybdenum catalysts, was still well below the recommended STY range. Carbon selectivity to $\mathrm{C}_{2}+$ oxygenates was only about $10 \%$ under these conditions.

The two Rh-based catalysts, $\mathrm{Rh} / \mathrm{Mn} /$ silica $\left(\mathrm{SiO}_{2}\right)$ and $\mathrm{Rh} / \mathrm{Mn} / \mathrm{Fe} / \mathrm{SiO}_{2}$, were very selective to $\mathrm{C}_{2}+$ oxygenates. The $\mathrm{Rh} / \mathrm{Mn} / \mathrm{Fe} / \mathrm{SiO}_{2}$ achieved higher $\mathrm{C}_{2}+$-oxygenate $\mathrm{STYs}$ under optimum conditions than any of the modified methanol and Mo-based catalysts tested at their optimum conditions and the FT catalysts at conditions that limited the total organic STYs to within the recommended range. The 
maximum achieved $\mathrm{C}_{2}+$-oxygenate $\mathrm{STY}$ (approximately $400 \mathrm{~g} / \mathrm{L}_{\text {cat }} / \mathrm{hr}$ ), however, was still below the recommended minimum. The carbon selectivity to $\mathrm{C}_{2}+$ oxygenates under this condition was approximately $24 \%$, which was significantly better than the FT catalysts. This Rh catalyst also was unique because it produced very few $\mathrm{C}_{1}+$ oxygenates or FT liquids. Based on these results, catalyst testing in 2007 focused on the Rh-based catalyst to examine the effects of other promoters besides iron (Fe) on catalyst performance. 



\subsection{Catalyst Testing}

The second set of catalyst testing, which was performed at Pacific Northwest National Laboratory (PNNL) in 2007, evaluated 22 promoters for the $\mathrm{RhMn} / \mathrm{SiO}_{2}$ catalysts including the Fe-promoted catalyst that was tested in previous catalyst screening. Repeat tests of unpromoted catalysts also were performed. The synthesis reactor system and the catalysts tested are described in this section.

\subsection{Synthesis Reactor System Description}

A bench-scale tubular reactor system was designed to operate at pressures up to $1200 \mathrm{psig}$ and temperatures up to $450^{\circ} \mathrm{C}$. The catalyst chamber was $1.67 \mathrm{~cm}$ long and $0.635 \mathrm{~cm}(0.25 \mathrm{in}$.) in diameter. A $0.159-\mathrm{cm}(1 / 16-i n$.$) outer diameter thermocouple sheath extended through the center of the reactor,$ creating an annulus-shaped catalyst chamber. Two thermocouples inside the sheath were spaced so one thermocouple was at the center of the catalyst bed and the other just upstream.

Figure 2.1 is a simplified diagram of the reactor system. During a portion of the testing, the reactor was oriented vertically in a clamshell furnace. During later tests, the clamshell furnace was replaced as the heat source by a hot oil circulating system. Heating the reactor with hot oil provided better temperature control because it could more efficiently remove the heat of reaction, thus preventing a thermal excursion when the carbon conversion was too high.

The syngas fed to the reactor was metered through a mass flow controller. The system also metered reducing gas $\left(10 \% \mathrm{H}_{2}\right.$ in nitrogen $\left.\left[\mathrm{N}_{2}\right]\right)$ and nitrogen $\mathrm{N}_{2}$ to the reactor during catalyst reduction. The raw product gas leaving the reactor was passed through one of two cold traps to condense liquids at $0^{\circ} \mathrm{C}$ and through a back-pressure regulator that controlled the system pressure. Gas flow was redirected from one trap to the other to isolate the former trap for liquid sample recovery.

The nominal feed rate to the reactor was determined by calibrating the mass flow controllers at system pressure before the tests, using a Bios DryCal flow meter downstream of the back-pressure flow regulator. Flow-meter readings were corrected for standard pressure and temperature. The flow meter also was used to monitor product gas flow rate downstream of the liquid sample traps during each test.

Product gas grab samples were obtained downstream of the back-pressure regulator in a line separate from that containing the bubble flow meter, as shown in Figure 2.1. The reactor inlet, catalyst bed, cold sample trap, ambient temperature, and the upstream gas and ambient pressures were monitored during tests.

Gas cylinders containing a specified syngas mixture were used in the tests. The gas mixture consisted of $\mathrm{H}_{2}, \mathrm{CO}, \mathrm{CO}_{2}$, and $\mathrm{N}_{2}$. Most of the tests conducted with the Rh-based catalysts used a gas that had a nominal $\mathrm{H}_{2}: \mathrm{CO}$ ratio of 1.8 with the ratio ranging from 1.7 to 1.9 . The nominal concentrations of $\mathrm{CO}_{2}$ and $\mathrm{N}_{2}$ were each $4 \%$ in the gas mixture. Variations in the $\mathrm{H}_{2}$ : $\mathrm{CO}$ ratios in the feed gas are attributed to variations in the composition of the individual gas cylinder mixtures that were supplied for the tests. 


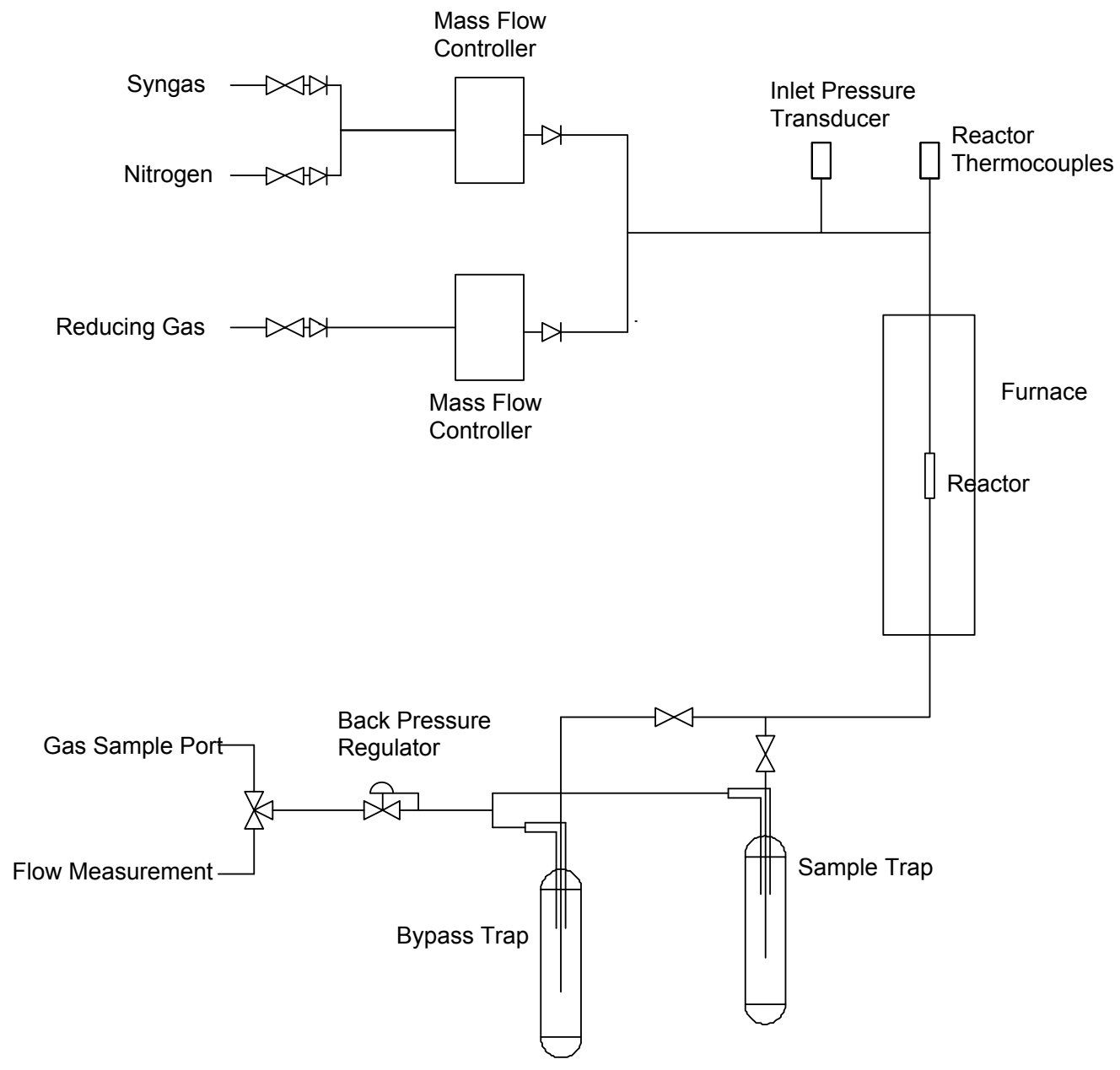

Figure 2.1. Simplified Diagram of Reactor System

\subsection{Catalyst Preparation}

The catalysts tested at PNNL during this testing phase are shown in Table 2.1, along with the labels used in this report. All of the catalysts used a baseline catalyst composition of $5.56 \% \mathrm{Rh}$ and $1.69 \% \mathrm{Mn}$ on $\mathrm{SiO}_{2}$. All promoters except $\mathrm{Li}$ were added at a concentration so that the $\mathrm{Rh}: \mathrm{Mn}: \mathrm{M}(\mathrm{M}=$ promoter $)$ atomic ratios were 1.00:0.57:0.10. The atomic ratios for Li were 1.00:0.57:0.30.

All catalysts except for $\mathrm{RhMnFe} / \mathrm{SiO}_{2} \mathrm{~B}$ used Davisil 645 high-surface-area $\mathrm{SiO}_{2}$ as the support and a two-step impregnation procedure using the incipient wetness technique. $\mathrm{The}^{\mathrm{SiO}_{2}}$ was pretreated by calcining at $500^{\circ} \mathrm{C}$ for 2 hours (ramping up at $5^{\circ} \mathrm{C} /$ min during heating and ramping down at $10^{\circ} \mathrm{C} / \mathrm{min}$ during cooling). The appropriate quantities of a rhodium nitrate solution $(10 \mathrm{wt} \% \mathrm{Rh}$ concentration in solution) and magnesium nitrate tetrahydrate were combined with enough deionized water to bring the total volume of the impregnation solution to the water adsorption pore volume of the support. The solution was impregnated onto the $\mathrm{SiO}_{2}$ in drop-wise fashion and then dried overnight at $110^{\circ} \mathrm{C}$.

A second impregnation was performed in a similar manner using an aqueous solution of the nitrate salt of the desired metal promoter followed by drying overnight at $110^{\circ} \mathrm{C}$. The dried catalyst was calcined at $400^{\circ} \mathrm{C}$ in air using a muffle furnace. 
Table 2.1. List of Catalysts Tested and Corresponding Labels

\begin{tabular}{|c|c|c|c|c|}
\hline Promoter & Label & Support & Rh Concentration, \% & Rh:Mn:M Ratio \\
\hline \multirow{3}{*}{$\begin{array}{l}\text { Baseline Catalyst } \\
\left(\mathrm{RhMn} / \mathrm{SiO}_{2}\right)\end{array}$} & $\mathrm{RhMn} / \mathrm{SiO}_{2} \mathrm{~A}$ & Davisil $645^{(\mathrm{c})}$ & 5.57 & $1.00: 0.57: 0.10$ \\
\hline & $\mathrm{RhMn} / \mathrm{SiO}_{2} *$ & Davisil 645 & 5.57 & $1.00: 0.57: 0.10$ \\
\hline & $\mathrm{RhMnFe} / \mathrm{SiO}_{2}{ }^{(\mathrm{a}, \mathrm{b})}$ & Davisil $645^{(\mathrm{c})}$ & 5.57 & $1.00: 0.57: 0.10$ \\
\hline \multirow[t]{2}{*}{$\mathrm{Fe}$} & $\mathrm{RhMnFe} / \mathrm{SiO}_{2} \mathrm{~A}^{(\mathrm{b})}$ & Davisil $645^{(\mathrm{c})}$ & 5.57 & $1.00: 0.57: 0.10$ \\
\hline & $\mathrm{RhMnFe} / \mathrm{SiO}_{2} \mathrm{~B}^{(\mathrm{b})}$ & Davisil LC150 & 5.57 & $1.00: 0.57: 0.10$ \\
\hline Co & $\mathrm{RhMnCo} / \mathrm{SiO}_{2}$ & Davisil $645^{(\mathrm{c})}$ & 5.57 & $1.00: 0.57: 0.10$ \\
\hline $\mathrm{Ni}$ & $\mathrm{RhMnNi} / \mathrm{SiO}_{2}{ }^{(\mathrm{b})}$ & Davisil $645^{(\mathrm{c})}$ & 5.57 & $1.00: 0.57: 0.10$ \\
\hline $\mathrm{Cu}$ & $\mathrm{RhMnCu} / \mathrm{SiO}_{2}$ & Davisil $645^{(\mathrm{c})}$ & 5.57 & $1.00: 0.57: 0.10$ \\
\hline \multirow{2}{*}{$\mathrm{Li}$} & $\mathrm{RhMnLi} / \mathrm{SiO}_{2}{ }^{(\mathrm{b})}$ & Davisil $645^{(\mathrm{c})}$ & 5.57 & $1.00: 0.57: 0.3$ \\
\hline & $\mathrm{RhMnLiA} / \mathrm{SiO}_{2}{ }^{(\mathrm{b})}$ & Davisil $645^{(\mathrm{c})}$ & 5.57 & $1.00: 0.57: 0.3$ \\
\hline \multirow{2}{*}{ Ir } & $\mathrm{RhMnIr} / \mathrm{SiO}_{2}$ & Davisil $645^{(\mathrm{c})}$ & 5.57 & $1.00: 0.57: 0.10$ \\
\hline & $\mathrm{RhMnIr} / \mathrm{SiO}_{2} \mathrm{~A}^{(\mathrm{b})}$ & Davisil $645^{(\mathrm{c})}$ & 5.57 & $1.00: 0.57: 0.10$ \\
\hline $\operatorname{Re}$ & $\mathrm{RhMnRe} / \mathrm{SiO}_{2}{ }^{(\mathrm{b})}$ & Davisil $645^{(\mathrm{c})}$ & 5.57 & $1.00: 0.57: 0.10$ \\
\hline Mo & $\mathrm{RhMnMo} / \mathrm{SiO}_{2}$ & Davisil $645^{(\mathrm{c})}$ & 5.57 & $1.00: 0.57: 0.10$ \\
\hline $\mathrm{Pd}$ & $\mathrm{RhMnPd} / \mathrm{SiO}_{2}$ & Davisil 645 & 5.57 & $1.00: 0.57: 0.52$ \\
\hline $\mathrm{Pt}$ & $\mathrm{RhMnPt} / \mathrm{SiO}_{2}$ & Davisil 645 & 5.57 & $1.00: 0.57: 0.10$ \\
\hline $\mathrm{Ru}$ & $\mathrm{RhMnRu} / \mathrm{SiO}_{2}$ & Davisil 645 & 5.57 & $1.00: 0.57: 0.10$ \\
\hline $\mathrm{Ga}$ & $\mathrm{RhMnGa} / \mathrm{SiO}_{2}$ & Davisil 645 & 5.57 & $1.00: 0.57: 0.10$ \\
\hline In & $\mathrm{RhMnIn} / \mathrm{SiO}_{2}$ & Davisil 645 & 5.57 & $1.00: 0.57: 0.10$ \\
\hline $\mathrm{Sn}$ & $\mathrm{RhMnSn} / \mathrm{SiO}_{2}$ & Davisil 645 & 5.57 & $1.00: 0.57: 0.10$ \\
\hline $\mathrm{Ge}$ & $\mathrm{RhMnGe} / \mathrm{SiO}_{2}$ & Davisil 645 & 5.57 & $1.00: 0.57: 0.10$ \\
\hline $\mathrm{Te}$ & $\mathrm{RhMnTe} / \mathrm{SiO}_{2}$ & Davisil 645 & 5.57 & $1.00: 0.57: 0.10$ \\
\hline $\mathrm{Bi}$ & $\mathrm{RhMnBi} / \mathrm{SiO}_{2}$ & Davisil 645 & 5.57 & $1.00: 0.57: 0.10$ \\
\hline $\mathrm{Pb}$ & $\mathrm{RhMnPb} / \mathrm{SiO}_{2}$ & Davisil 645 & 5.57 & $1.00: 0.57: 0.10$ \\
\hline $\mathrm{Au}$ & $\mathrm{RhMnAu} / \mathrm{SiO}_{2}$ & Davisil 645 & 5.57 & $1.00: 0.57: 0.10$ \\
\hline V & $\mathrm{RhMnV} / \mathrm{SiO}_{2}$ & Davisil 645 & 5.57 & $1.00: 0.57: 0.10$ \\
\hline Cs & $\mathrm{RhMnCs} / \mathrm{SiO}_{2}$ & Davisil 645 & 5.57 & $1.00: 0.57: 0.10$ \\
\hline $\mathrm{Ag}$ & $\mathrm{RhMnAg} / \mathrm{SiO}_{2}$ & Davisil 645 & 5.57 & $1.00: 0.57: 0.10$ \\
\hline
\end{tabular}

(a) Sample tested in previous screening tests (Gerber et al. 2007).

(b) Test conducted using furnace instead of hot oil circulation.

(c) Silica was obtained from Altech, Inc.

The preparation of the $\mathrm{RhMnFeB}$ catalyst used Davisil $150 \mathrm{LC} \mathrm{SiO}_{2}$ as the support and a single impregnation consisting of an aqueous solution of $\mathrm{Rh}, \mathrm{Mn}$, and Fe nitrate salts. The $\mathrm{RhMn} / \mathrm{SiO}_{2} \mathrm{~A}$ and $\mathrm{RnMn} / \mathrm{SiO}_{2}$ * catalysts also consisted of a single impregnation because no additional metals were added to the catalysts.

Prior to conducting the tests, the calcined catalysts were loaded into the reactor and reduced using a $10 \% \mathrm{H}_{2}$-in- $\mathrm{N}_{2}$ gas mixture. The $\mathrm{RhMn} / \mathrm{SiO}_{2}, \mathrm{RhMnFe} / \mathrm{SiO}_{2}, \mathrm{RhMnFe} / \mathrm{SiO}_{2} \mathrm{~A}, \mathrm{RhMnFe} / \mathrm{SiO}{ }_{2} \mathrm{~B}$, and $\mathrm{RhMnLi} / \mathrm{SiO}_{2}$ catalysts were heated in the reducing atmosphere to $220^{\circ} \mathrm{C}$ at $2.5^{\circ} \mathrm{C} / \mathrm{min}$, held at that temperature for 1 hour, and then heated from $220^{\circ} \mathrm{C}$ to $260^{\circ} \mathrm{C}$ at $1{ }^{\circ} \mathrm{C} / \mathrm{min}$ and held at that temperature 
overnight. All other catalysts were heated in the reducing atmosphere to $220^{\circ} \mathrm{C}$ at $2.5^{\circ} \mathrm{C} / \mathrm{min}$ and held that temperature for 1 hour, heated from $220^{\circ} \mathrm{C}$ to $260^{\circ} \mathrm{C}$ at $1^{\circ} \mathrm{C} / \mathrm{min}$ and held at that temperature for 8 hours, and heated to approximately $350^{\circ} \mathrm{C}$ at $1.5^{\circ} \mathrm{C} / \mathrm{min}$ and held at that temperature for 2 hours.

\subsection{Testing Procedure}

During a typical test series, a measured volume of catalyst was loaded into the reactor, and its net weight was determined. The reactor was placed in the reactor system, and was reduced in situ at atmospheric pressure. The reactor was cooled after catalyst reduction, and the desired syngas feed rate and pressure were established. The reactor was heated slowly to a temperature at which the reaction rate was significant and maintained at that temperature for at least 24 hours to allow the catalyst to age. The product stream was directed through one of the cold traps during this time. After aging the catalyst, the product stream was redirected through the other cold trap for a period sufficient for at least 10 bed volumes of gas feed (based on the operating pressure and gas feed rate) to pass through the cold trap. This period of time provides a representative gas sample and a sufficiently large liquid sample for subsequent analysis. The operating conditions were recorded before sampling with two or more grab samples of product gas obtained and analyzed in a gas chromatograph (GC) along with a feed gas sample and a calibration gas sample. The liquid recovered from the cold trap was weighed and, if two phases were present, separated into an aqueous phase and an organic phase. The weighed organic phase was not analyzed and was assumed to have a composition comparable to hexane for purposes of a carbon balance. The weighed aqueous phase was analyzed using a high-pressure liquid chromatograph (HPLC) to quantify the $\mathrm{C}_{1}-\mathrm{C}_{5}$ oxygenates, which principally were alcohols, acids, aldehydes, esters, and any other products associated with significant peaks identified by the HPLC. After sampling, a new set of conditions (temperature and feed rate) was established and another cold trap sample collected at the new conditions. This procedure was repeated until a representative set of conditions was obtained to evaluate catalyst performance in terms of STY, carbon selectivity, and single-pass carbon conversion. In most cases, tests progressively advanced to higher temperatures with one or more space velocities examined during each test. In some tests, previous conditions were re-examined to determine whether further catalyst ageing during testing affected the performance of the catalyst.

To calculate a representative average outlet flow rate during a sample collection period, an $\mathrm{N}_{2}$ balance was used with the calibrated feed flow rates. The product gas flow rate downstream of the cold trap was monitored and recorded for estimating the product gas flow rate and to provide a rough check on the accuracy of the calculated flow using a nitrogen balance. Carbon balances using this method were approximately $\pm 10 \%$. 


\subsection{Reactor System Performance}

During earlier testing, it was determined that the measured outlet flow rate at a particular point in time was not always representative of the average flow rate during liquid sample collection in the cold trap because of slow fluctuations over irregular periods of several minutes to several hours. These fluctuations are attributed to mass flow controller flow-rate oscillations, relatively small fluctuations in the reactor pressure, and transient changes in catalyst reactivity. Flow rate was measured four or more times over a period of approximately 1 hour prior to sampling, and then averaged to mitigate the effects of the mass flow controller. After it was determined that the temperature sensitivity of the back-pressure regulator was the likely cause of periodic pressure changes, the back-pressure regulator was wrapped with heat tape and maintained at a constant temperature $\left(35^{\circ} \mathrm{C}\right)$ to minimize pressure fluctuations.

Longer periods of temperature fluctuation were found to occur during testing. These fluctuations were attributed to the behavior of the catalyst. Some of the change could be attributed to slow deactivation of the catalyst that appeared to be more pronounced at temperatures above $325^{\circ} \mathrm{C}$. However, it was also found that when the more reactive catalysts were operated at reaction rates approaching the limits of the reactor furnace to remove excess heat, small changes in reactor temperature could cause large fluctuations in the catalyst bed temperature. These excursions lasted anywhere from a few hours to a half a day and in some cases produced a periodic temperature cycle that ranged as much as $40^{\circ} \mathrm{C}$ over time. This phenomenon has been reported in the literature for FT catalysts (Tsotsis, Rao, and Polinski 1982). If temperature fluctuations could be maintained within a approximately $12^{\circ} \mathrm{C}$ temperature range for a sufficiently long period of time, data were collected and a sample was taken, using the average catalyst temperature during the sampling period as the basis for performance comparison to other catalysts. If temperature fluctuations were too great, then a different set of conditions was sought that could produce acceptable temperature fluctuations. Consequently, test conditions at all temperatures were not evaluated when the furnace was used. By switching to reactor heating with a hot oil circulating system, which was more efficient for removing excess heat, catalyst temperatures excursions were reduced to a couple of degrees for most tests, and all temperatures could be evaluated then. 



\subsection{Test Results}

The primary purpose of the testing described in this report was to determine whether adding different promoters to an $\mathrm{Rh}-\mathrm{Mn} / \mathrm{SiO}_{2}$ catalyst would achieve significant improvements in $\mathrm{C}_{2}$--oxygenate $\mathrm{STY}$ and selectivity. The addition of promoters to the $\mathrm{RhMn} / \mathrm{SiO}_{2}$ catalyst was conducted to evaluate their influence on the productivity and/or product selectivity of the catalyst. For the purpose of comparison, all catalysts except for the Li-promoted catalysts were added to the $\mathrm{RhMn} / \mathrm{SiO} 2$ catalyst so that the $\mathrm{Rh}: \mathrm{Mn}:$ Promoter metal ratio was constant at 1.00/0.565/0.0986, with a $\mathrm{Rh}$ metal concentration of 5.65\% on the $\mathrm{SiO}_{2}$ support. The Li catalyst used a 1.00:0.565:0.30 ratio with the same $\mathrm{Rh}$ concentration. Table A.1 in Appendix A summarizes the test results. The results are discussed in more detail in Appendix $\mathrm{B}$ according to the type of promoter added to the $\mathrm{RhMn} / \mathrm{SiO}_{2}$ catalyst.

\subsection{General Results}

It is clear that even though the promoters were added at a nearly tenfold lower concentration relative to $\mathrm{Rh}$ (with the exception of $\mathrm{Li}$ promoter), their effects on catalyst activity ranged from beneficial to very detrimental. For example, the Ir-promoted catalyst had a maximum $\mathrm{C}_{2}+$-oxygenate STY of approximately $800 \mathrm{~g} / \mathrm{L}_{\text {cat }} / \mathrm{hr}$ that was $78 \%$ better that the highest STY achieved with the any of the unpromoted catalysts tested $\left(450 \mathrm{~g} / \mathrm{L}_{\text {cat }} / \mathrm{hr}\right.$ for the $\mathrm{RhMn} / \mathrm{SiO}_{2} \mathrm{~A}$ catalyst), while the catalysts promoted by germanium $(\mathrm{Ge})$, tellurium $(\mathrm{Te})$, lead $(\mathrm{Pb})$, and bismuth $(\mathrm{Bi})$ were essentially inactive under similar testing conditions.

As previously discussed, temperature control of the more active catalysts using a clamshell furnace to heat the reactor was difficult, if not impossible, to maintain when the carbon conversion was above approximately $30 \%$. Replacing the furnace with a hot oil circulating system, gave much better control for the more active catalysts, although control was still difficult for some of the more active catalysts promoted by Ir, Pt, Sn, Au, and Mo. It was noted that all of these catalysts also produced significant quantities of hydrocarbon liquids, which may be related to the difficulty with temperature control (there were other catalysts heated with the furnace that produced significant quantities of hydrocarbon liquids). In tests conducted with the Ir-promoted catalyst using both the furnace and the oil heating system, $\mathrm{C}_{2}+$-oxygenate STYs were higher when the oil heating system was used. This is attributed to the improved temperature control of the oil heating system that allowed tests to be conducted at temperatures and gas flow rates at which the maximum STY could be achieved, but was unattainable using the furnace. This suggests that similar improvements in the STYs may be possible for the more active catalysts (catalysts promoted by $\mathrm{Li}, \mathrm{Ni}$, and $\mathrm{Fe}$ ) that were tested only with the furnace and that experienced similar difficulties obtaining stable conditions at the expected optimum test conditions.

Another point of note during testing was the source of the $\mathrm{SiO}_{2}$. Although all of the tests were performed with Davisil $645 \mathrm{SiO}_{2}$, different sources of the $\mathrm{SiO}_{2}$ were used in preparing the catalysts. Most of the earlier tested catalysts used an initial lot of the Davisil $\mathrm{SiO}_{2}$ obtained from Altech Inc., (i.e., the catalysts promoted by $\mathrm{Fe}, \mathrm{Li}, \mathrm{Ir}, \mathrm{Ni}, \mathrm{Re}, \mathrm{Cu}, \mathrm{Co}$, and $\mathrm{Mo}$ ). The other catalysts used $\mathrm{SiO}_{2}$ from more recent lots obtained from Fisher Scientific. The catalysts supported on the $\mathrm{SiO}_{2}$ supplied by Fisher Scientific had a tendency to produce significant quantities of methanol while those supported on $\mathrm{SiO}_{2}$ from Altech did not. For example, five of the nine catalysts tested (the catalysts promoted by $\mathrm{Ir}, \mathrm{Re}, \mathrm{Fe}$, $\mathrm{Ni}$, and $\mathrm{Co}$ ) using $\mathrm{SiO}_{2}$ supplied by Altech had $\mathrm{MeOH}$ selectivities less that $1 \%$ at all conditions tested, and only three of the nine catalysts (those promoted by $\mathrm{Li}, \mathrm{Cu}$, and $\mathrm{Mo}$ ) had selectivities greater than $2 \%$. 
None of these catalysts had selectivities greater than $3 \%$, and the unpromoted catalyst had a maximum selectivity of $1.5 \%$. On the other hand, only 3 of the 15 catalysts tested (the Pt-, Ru-, and Cs-promoted catalysts) using the Fischer Scientific source of $\mathrm{SiO}_{2}$ had carbon selectivities to $\mathrm{MeOH}$ less than $1 \%$, while nine of the catalysts (the catalysts promoted by $\mathrm{Ga}, \mathrm{In}, \mathrm{Ge}, \mathrm{Bi}, \mathrm{Pb}, \mathrm{Au}, \mathrm{V}$, silver [Ag], and the unpromoted catalyst) had carbon selectivities to methanol greater than 5\% (the Te-promoted catalyst was inactive while the palladium [Pd], and Sn catalysts had selectivities between 1 and 3\%, respectively). Of particular note was the difference in the $\mathrm{MeOH}$ selectivity for the unpromoted catalysts prepared using the different catalyst sources with the catalyst prepared from the Altech-supplied $\mathrm{SiO}_{2}$ producing a maximum $\mathrm{MeOH}$ selectivity of $1.5 \%$ tests, and the catalysts prepared from the Fisher Scientific-supplied $\mathrm{SiO}_{2}$ achieving a maximum 7\% selectivity. Under similar test conditions, the catalysts supported on $\mathrm{SiO}_{2}$ obtained from Fisher Scientific also had lower $\mathrm{C}_{2}+$-oxygenate $\mathrm{STYs}$ than those supported on the $\mathrm{SiO}_{2}$ obtained from Altech Inc.

Taking into consideration the caveats described above in testing, several general trends were observed for the catalysts during testing with few exceptions:

- Carbon conversion increased with increasing temperature and decreased with increasing space velocity. Notable exceptions were the Mo-, Ru-, and V-promoted catalysts that showed clear evidence of significant catalyst deactivation at higher temperatures.

- When catalysts were heated to between 300 and $325^{\circ} \mathrm{C}$, the catalyst activity for $\mathrm{C}_{2}+$ oxygenates decrease to varying degrees, accompanied by reduced hydrocarbon chain growth (fewer hydrocarbon liquids and more methane). The degree of deactivation and the temperature at which it occurred varied between the different catalysts tested. (Tests to determine the long-term effect of time at any one temperature on catalyst activity were not performed).

- The maximum $\mathrm{C}_{2}+$-oxygenate STYs achieved with any catalyst occurred between 300 and $325^{\circ} \mathrm{C}$, with the gas hourly space velocity (GHSV) adjusted when necessary to maintain carbon conversion ranges between approximately 30 and $40 \%$. Higher carbon selectivity to hydrocarbons at higher temperatures resulted in lower $\mathrm{C}_{2}+$-oxygenate STYs.

- The highest carbon selectivity to $\mathrm{C}_{2}+$ oxygenates occurred at the lowest reaction temperatures and accompanying lowest STYs.

- The lowest carbon selectivity to $\mathrm{C}_{2}+$ oxygenates occurred at the highest reaction temperatures because of high carbon conversion to hydrocarbons under these conditions.

- The selectivity of all oxygenates to $\mathrm{C}_{2}+$ alcohols generally increased with increasing temperature. Notable exceptions were the Ga- and In-promoted catalysts, which maintained a constant ratio of approximately $70 \% \mathrm{C}_{2}+$ alcohols:all oxygenates at temperatures above $275^{\circ} \mathrm{C}$, and the cesium (Cs)promoted catalyst, which decreased to a value of approximately $10 \%$ at temperatures above $275^{\circ} \mathrm{C}$.

\subsection{Comparison of Catalyst Performance}

Two bases were used to compare the performance of the catalysts: 1) the conditions under which the maximum STYs were achieved, and 2) at a common set of operating conditions $\left(300^{\circ} \mathrm{C}, 11,000 \mathrm{~L} / \mathrm{L}_{\text {cat }} / \mathrm{hr}\right)$. The results for conditions under which the maximum STYs were achieved acknowledges the possibility that the different test conditions may be needed to maximize STYs because of differences in the interplay among temperature, space velocity, carbon conversion, and selectivity to $\mathrm{C}_{2}+$ oxygenates. Comparison of catalyst performance under a common set of conditions provides a common basis for those comparing 
carbon conversion and selectivity to products. The chosen set of conditions was selected because better catalysts also performed very well under these conditions, and in most cases, the catalysts had not undergone significant deactivation because of operating at higher temperatures.

Table 4.1 lists the test conditions at which the maximum $\mathrm{C}_{2}+$-oxygenate STY was achieved for the various promoters along with the corresponding carbon and CO conversions and liquid-product STYs. The STYs under these conditions are compared in Figure 4.1. In most cases, the maximum STY was obtained at a space velocity of $11,000 \mathrm{~L} / \mathrm{L}_{\text {cat }} / \mathrm{hr}$. Exceptions were the RhMnIrA and RhMnAu catalysts. These catalysts achieved their highest STYs at a space velocity of $15,000 \mathrm{~L} / \mathrm{L}_{\text {cat }} / \mathrm{hr}$ because the higher space velocity was required to maintain the carbon conversion at an acceptable level. The carbon selectivities to various products are listed and compared in Tables 4.2 and Figure 4.2, respectively. Tables 4.3 and 4.4 and Figures 4.3 and .4 .4 list and compare similar performance parameters for catalysts tested at the chosen conditions $\left(300^{\circ} \mathrm{C}\right.$ and $\left.11,000 \mathrm{~L} / \mathrm{L}_{\text {cat }} / \mathrm{hr}\right)$.

It can be seen from Figure 4.1 that the $\mathrm{Rh}-\mathrm{Mn} / \mathrm{SiO}_{2}$ catalysts promoted with $\mathrm{Ir}, \mathrm{Pt}, \mathrm{Au}, \mathrm{Li}, \mathrm{Ni}, \mathrm{Mo}$, and $\mathrm{Ru}$, had $\mathrm{C}_{2}+$-oxygenate STYs equal to or greater than that achieved with the baseline $\mathrm{Rh}-\mathrm{Mn} / \mathrm{SiO}$ catalyst (shown by the dotted line in Figure 4.1). The Ir-promoted catalyst had the highest STY achieved of all of the catalysts tested (approximately $800 \mathrm{~g} / \mathrm{L}_{\mathrm{cat}} / \mathrm{hr}$ ), followed by the Pt-promoted catalyst (approximately $660 \mathrm{~g} / \mathrm{L}_{\text {cat }} / \mathrm{hr}$ ). The Au-, Li-, Ni-, and Mo-promoted catalysts had similar STYs, while the $\mathrm{Ru}$-promoted catalyst was about the same as the unpromoted catalyst. While the Ni- and Li-promoted catalyst did not have STYs as high as the Ir catalyst, it should be noted that these catalysts were not tested with the hot oil heating system. Consequently, it is possible that they would have much higher STYs if the oil heated reactor were used, as was experienced by the Ir-promoted catalyst.

All of these catalysts displayed at least some degree of deactivation at the high temperatures tested. In particular, the Mo- and Ru-promoted catalysts experienced significant loss of activity at temperatures above $300^{\circ} \mathrm{C}$ so these catalysts are no longer under consideration. The Li- and Ni-promoted catalysts also experience significant deactivation, but this is attributed to catalyst temperatures reaching levels greater than 375 and $350^{\circ} \mathrm{C}$, respectively, because of poor heat management by the furnace. While the Li-promoted catalyst lost most of its activity, it was recovered by operating at higher temperatures, although the selectivity to oxygenates was reduced at the higher temperature. Both of these catalysts appeared to have much better thermal stability than the $\mathrm{Ru}$ - and Mo- promoted catalysts. The Ir- and Pt-promoted catalysts also were more stable than the $\mathrm{Ru}$ - and Mo-promoted catalysts.

Figure 4.3 shows that when compared on a common basis, the Ir-promoted catalyst is clearly the most active catalysts with respect to production of $\mathrm{C}_{2}+$ oxygenates. The Pt-promoted catalyst is comparable to the Au-, Li-, and Mo-promoted catalysts under these conditions, while the Ni-promoted catalyst is less productive than the baseline catalyst. There also was rearrangement in the ranking of some of the less reactive catalysts.

Tables 4.2 and 4.4 summarize the carbon selectivity to various liquid products for the catalysts under the conditions listed in Tables 4.1 and 4.3, respectively, while Figures 4.2 and 4.4 compare the selectivity to all products under the conditions listed in Tables 5.1 and 5.3, respectively. It can be seen that the Li-promoted catalyst had the highest carbon selectivity to $\mathrm{C}_{2}+$ oxygenates of all of the catalysts at the conditions where they achieved their maximum STYs and at the common testing conditions $\left(300^{\circ} \mathrm{C}\right.$, $11,000 \mathrm{~L} / \mathrm{L}_{\text {cat }} / \mathrm{hr}$ ). However, it is noteworthy that its selectivity for $\mathrm{C}_{2}+$ oxygenates to alcohols also was one of the lowest among the catalysts tested. 
Table 4.1. Comparison of Carbon Conversions and STYs of Promoted $\mathrm{RhMn} / \mathrm{SiO}_{2}$ Catalysts at Conditions Maximizing $\mathrm{C}_{2}+-\mathrm{Oxygenate} \mathrm{STYs}$

\begin{tabular}{|c|c|c|c|c|c|c|c|c|c|c|c|}
\hline \multirow[b]{2}{*}{ Catalyst } & \multirow[b]{2}{*}{$\begin{array}{c}\text { Space } \\
\text { Velocity, } \\
\text { L/L/hr }\end{array}$} & \multirow[b]{2}{*}{$\begin{array}{l}\text { Temp., } \\
{ }^{\circ} \mathrm{C}\end{array}$} & \multirow[b]{2}{*}{$\begin{array}{c}\text { Carbon } \\
\text { Conv., } \\
\%\end{array}$} & \multirow[b]{2}{*}{$\begin{array}{c}\mathrm{CO} \\
\text { Conv., } \\
\%\end{array}$} & \multirow[b]{2}{*}{$\begin{array}{c}\text { CO Conv. } \\
\text { to } \mathrm{CO}_{2} \\
\%\end{array}$} & \multicolumn{5}{|c|}{$\mathrm{STY}, \mathrm{g} / \mathrm{mL}_{\mathrm{cat}} / \mathrm{hr}$} & \multirow[b]{2}{*}{$\begin{array}{c}\text { Carbon } \\
\text { Balance } \\
\left(\mathrm{C}_{\text {out }} / \mathrm{C}_{\text {in }}\right) \\
\% \\
\%\end{array}$} \\
\hline & & & & & & $\mathrm{MEOH}$ & $\begin{array}{c}\mathrm{C}_{2}+ \\
\text { Alcohols }\end{array}$ & $\begin{array}{c}\text { Other } \mathrm{C}_{2}+ \\
\text { Oxygenates }\end{array}$ & $\begin{array}{c}\mathrm{HC} \\
\text { Liquids }\end{array}$ & $\begin{array}{c}\text { Total } \\
\text { Liquids }\end{array}$ & \\
\hline RhMnIrA & 15,000 & 325 & 37.8 & 42.7 & 0.42 & 0.007 & 0.210 & 0.596 & 0.013 & 0.825 & 99.44 \\
\hline $\mathrm{RhMnPt}$ & 11,000 & 313 & 40.3 & 45.3 & -0.05 & 0.003 & 0.108 & 0.556 & 0.052 & 0.720 & 99.02 \\
\hline $\mathrm{RhMnAu}$ & 15,000 & 314 & 24.8 & 27.9 & -0.15 & 0.008 & 0.114 & 0.386 & 0.013 & 0.520 & 97.96 \\
\hline $\mathrm{RhMnLiA}^{(\mathrm{a})}$ & 11,000 & 305 & 24.5 & 28.2 & 0.67 & 0.006 & 0.099 & 0.386 & 0.019 & 0.510 & 97.13 \\
\hline $\mathrm{RhMnNi}^{(\mathrm{a})}$ & 11,000 & 325 & 34.1 & 39.1 & 1.00 & 0.000 & 0.169 & 0.307 & 0.002 & 0.478 & 99.78 \\
\hline RhMnMo & 12,000 & 298 & 30.1 & 38.9 & 4.95 & 0.016 & 0.165 & 0.306 & 0.027 & 0.514 & 98.49 \\
\hline $\mathrm{RhMnRu}$ & 11,000 & 300 & 43.4 & 49.6 & 0.26 & 0.000 & 0.069 & 0.372 & 0.139 & 0.581 & 102.25 \\
\hline RhMnA & 11,000 & 324 & 32.6 & 37.2 & 0.60 & 0.006 & 0.123 & 0.316 & 0.007 & 0.452 & 98.09 \\
\hline RhMn* & 11,000 & 299 & 21.4 & 24.1 & -0.12 & 0.046 & 0.149 & 0.214 & 0.000 & 0.409 & 96.33 \\
\hline $\mathrm{RhMnFeB}^{(\mathrm{a})}$ & 11,000 & 325 & 35.3 & 41.8 & 1.55 & 0.011 & 0.195 & 0.168 & 0.015 & 0.388 & 97.69 \\
\hline RhMnSn & 11,000 & 325 & 21.6 & 23.8 & -0.51 & 0.013 & 0.158 & 0.197 & 0.017 & 0.385 & 98.99 \\
\hline RhMnCo & 11,000 & 307 & 29.4 & 33.1 & 0.02 & 0.005 & 0.085 & 0.270 & 0.001 & 0.361 & 95.37 \\
\hline $\mathrm{RhMnPd}^{*}$ & 12,000 & 300 & 16.7 & 18.2 & -0.56 & 0.009 & 0.072 & 0.228 & 0.000 & 0.310 & 96.99 \\
\hline RhMnV & 15,000 & 315 & 21.6 & 25.0 & 0.62 & 0.095 & 0.132 & 0.143 & 0.003 & 0.372 & 97.86 \\
\hline $\operatorname{RhMnRe}^{(a)}$ & 11,000 & 324 & 38.0 & 44.8 & 1.63 & 0.009 & 0.154 & 0.102 & 0.047 & 0.313 & 94.60 \\
\hline RhMnGa & 11,000 & 345 & 17.8 & 20.9 & 0.83 & 0.064 & 0.154 & 0.034 & 0.000 & 0.252 & 99.26 \\
\hline $\mathrm{RhMnCu}$ & 11,000 & 315 & 9.5 & 10.4 & -0.23 & 0.012 & 0.071 & 0.076 & 0.000 & 0.159 & 97.96 \\
\hline RhMnIn & 11,000 & 345 & 8.7 & 10.2 & 0.30 & 0.043 & 0.119 & 0.019 & 0.000 & 0.181 & 98.52 \\
\hline RhMnCs & 11,000 & 325 & 10.9 & 12.4 & 0.15 & 0.004 & 0.012 & 0.104 & 0.000 & 0.120 & 98.74 \\
\hline RhMnAg & 11,000 & 325 & 12.2 & 13.7 & -0.18 & 0.030 & 0.064 & 0.042 & 0.000 & 0.136 & 101.46 \\
\hline $\mathrm{RhMnPb}$ & 11,000 & 325 & 1.2 & 1.9 & 0.52 & 0.006 & 0.006 & 0.003 & 0.000 & 0.015 & 103.53 \\
\hline RhMnGe & 11,000 & 326 & 1.5 & 2.1 & 0.43 & 0.018 & 0.008 & 0.001 & 0.000 & 0.027 & 99.85 \\
\hline $\mathrm{RhMnBi}$ & 11,000 & 345 & 0.9 & 1.0 & -0.01 & 0.005 & 0.004 & 0.004 & 0.000 & 0.013 & 99.12 \\
\hline RhMnTe & 11,000 & 326 & 0 & 0 & 0 & 0 & 0 & 0 & 0 & 0 & 98.29 \\
\hline
\end{tabular}




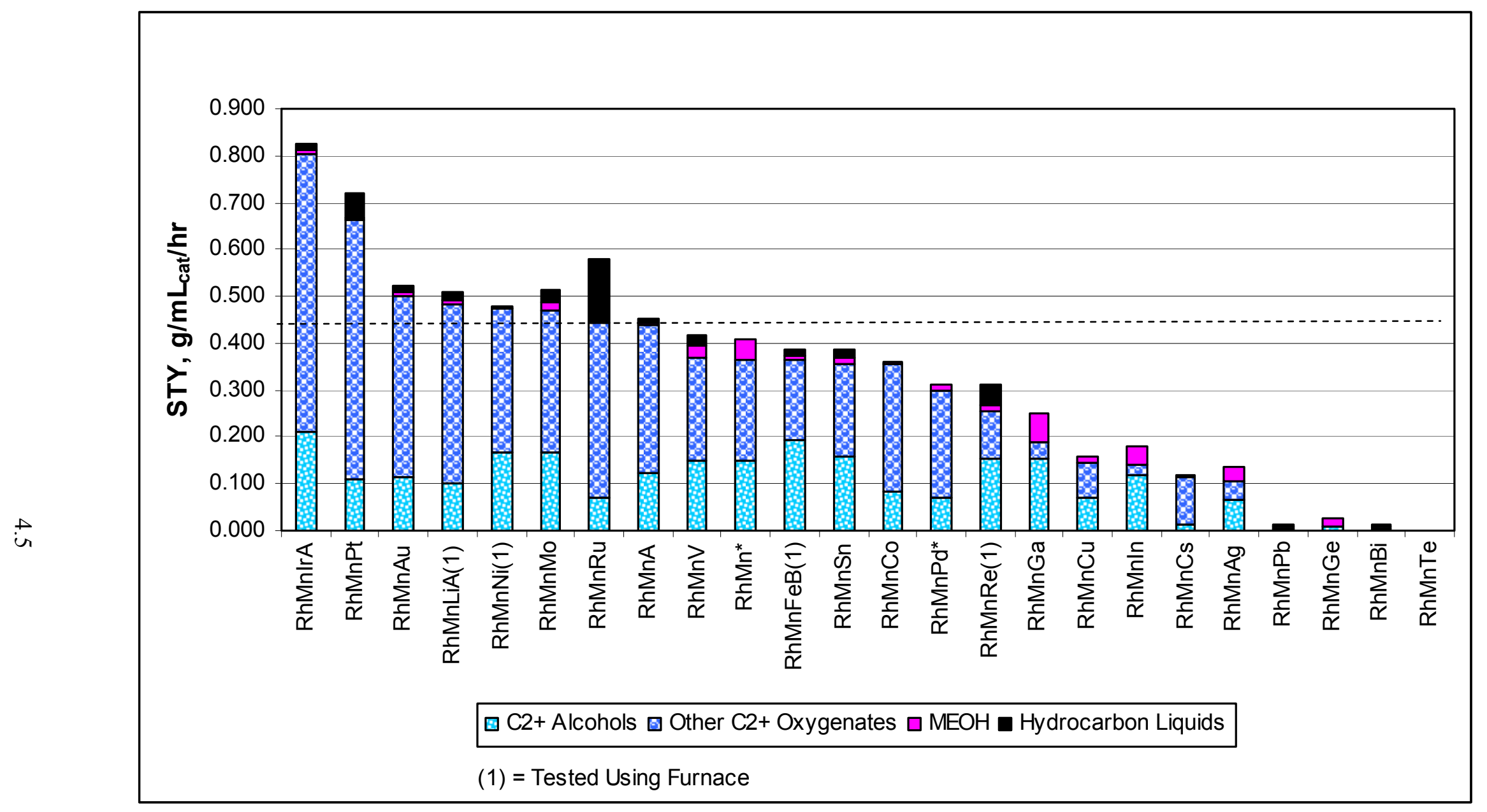

Figure 4.1. STYs of Promoted Catalysts at Conditions for Maximum STYs 
Table 4.2. Comparison of Carbon Selectivities to Products of Promoted $\mathrm{RhMn} / \mathrm{SiO}_{2}$ Catalysts at Conditions Maximizing $\mathrm{C}_{2}+-\mathrm{Oxygenate} \mathrm{STY} s$

\begin{tabular}{|c|c|c|c|c|c|c|c|c|c|c|}
\hline \multirow[b]{2}{*}{ Catalyst } & \multirow{2}{*}{$\begin{array}{c}\text { Space } \\
\text { Velocity, } \\
\text { L/L/hr }\end{array}$} & \multirow[b]{2}{*}{$\begin{array}{c}\text { Temp., } \\
{ }^{\circ} \mathrm{C}\end{array}$} & \multirow{2}{*}{$\begin{array}{c}\text { Carbon } \\
\text { Conv., } \\
\% \\
\end{array}$} & \multicolumn{7}{|c|}{ Carbon Selectivity (C-Mol\%) } \\
\hline & & & & $\mathrm{MEOH}$ & $\begin{array}{c}\text { Other } \mathrm{C}_{1} \\
\text { Oxygenates }\end{array}$ & $\begin{array}{c}\mathrm{C}_{2}+ \\
\text { Alcohols }\end{array}$ & $\begin{array}{c}\text { Other } \mathrm{C}_{2}+ \\
\text { Oxygenates }\end{array}$ & Methane & $\begin{array}{c}\text { Higher HC } \\
\text { Gases }\end{array}$ & $\begin{array}{l}\text { Higher HC } \\
\text { Liquids (est) }\end{array}$ \\
\hline RhMnIrA & 15,000 & 325 & 37.8 & 0.25 & 0.00 & 10.25 & 28.32 & 46.66 & 13.52 & 0.99 \\
\hline $\mathrm{RhMnPt}$ & 11,000 & 313 & 40.3 & 0.14 & 0.00 & 6.74 & 32.41 & 37.93 & 17.76 & 5.02 \\
\hline $\mathrm{RhMnAu}$ & 15,000 & 314 & 24.8 & 0.45 & 0.00 & 8.62 & 27.89 & 46.96 & 14.57 & 1.50 \\
\hline $\operatorname{RhMnLiA}^{(\mathrm{a})}$ & 11,000 & 305 & 24.5 & 0.42 & 0.00 & 10.32 & 36.55 & 34.90 & 14.66 & 3.14 \\
\hline $\mathrm{RhMnNi}^{(\mathrm{a})}$ & 11,000 & 325 & 34.1 & 0.00 & 0.00 & 11.86 & 20.60 & 56.10 & 11.26 & 0.18 \\
\hline RhMnMo & 12,000 & 298 & 30.1 & 0.88 & 0.00 & 13.45 & 22.79 & 44.18 & 15.31 & 3.38 \\
\hline $\mathrm{RhMnRu}$ & 11,000 & 300 & 43.4 & 0.00 & 0.00 & 4.04 & 20.29 & 32.06 & 31.25 & 12.37 \\
\hline RhMnA & 11,000 & 324 & 32.6 & 0.34 & 0.00 & 9.25 & 22.90 & 55.19 & 11.54 & 0.78 \\
\hline $\mathrm{RhMn*}$ & 11,000 & 299 & 21.4 & 3.72 & 0.00 & 17.29 & 23.54 & 43.76 & 11.69 & 0.00 \\
\hline $\mathrm{RhMnFeB}^{(\mathrm{a})}$ & 11,000 & 325 & 35.3 & 0.53 & 0.00 & 13.66 & 10.97 & 57.16 & 16.06 & 1.61 \\
\hline $\mathrm{RhMnSn}$ & 11,000 & 325 & 21.6 & 1.07 & 0.00 & 18.44 & 21.07 & 43.60 & 12.74 & 3.08 \\
\hline RhMnCo & 11,000 & 307 & 29.4 & 0.33 & 0.00 & 7.25 & 21.65 & 50.20 & 20.48 & 0.10 \\
\hline $\mathrm{RhMnPd}^{*}$ & 12,000 & 300 & 16.7 & 0.93 & 0.00 & 10.34 & 29.73 & 45.44 & 13.56 & 0.00 \\
\hline RhMnV & 15,000 & 315 & 21.6 & 5.80 & 0.00 & 11.49 & 12.18 & 56.41 & 13.76 & 0.37 \\
\hline $\mathrm{RhMnRe}^{(\mathrm{a})}$ & 11,000 & 324 & 38.0 & 0.40 & 0.00 & 9.72 & 6.09 & 62.07 & 17.08 & 4.63 \\
\hline $\mathrm{RhMnGa}$ & 11,000 & 345 & 17.8 & 6.27 & 0.00 & 21.38 & 4.24 & 57.03 & 11.08 & 0.00 \\
\hline $\mathrm{RhMnCu}$ & 11,000 & 315 & 9.5 & 2.19 & 0.00 & 18.76 & 18.97 & 54.82 & 5.26 & 0.00 \\
\hline RhMnIn & 11,000 & 345 & 8.7 & 8.45 & 0.00 & 33.84 & 4.94 & 47.95 & 4.81 & 0.00 \\
\hline RhMnCs & 11,000 & 325 & 10.9 & 0.66 & 0.00 & 2.83 & 21.18 & 53.24 & 22.08 & 0.00 \\
\hline $\mathrm{RhMnAg}$ & 11,000 & 325 & 12.2 & 4.31 & 0.00 & 13.20 & 8.13 & 64.13 & 10.23 & 0.00 \\
\hline $\mathrm{RhMnPb}$ & 11,000 & 325 & 1.2 & 7.91 & 0.68 & 12.36 & 6.08 & 57.95 & 15.02 & 0.00 \\
\hline $\mathrm{RhMnGe}$ & 11,000 & 326 & 1.5 & 22.09 & 0.00 & 13.70 & 1.15 & 63.06 & 0.00 & 0.00 \\
\hline RhMnBi & 11,000 & 345 & 0.9 & 9.69 & 0.04 & 11.29 & 8.96 & 62.14 & 8.94 & 0.00 \\
\hline RhMnTe & 11,000 & 326 & NA & NA & NA & NA & NA & $\mathrm{NA}$ & NA & NA \\
\hline
\end{tabular}




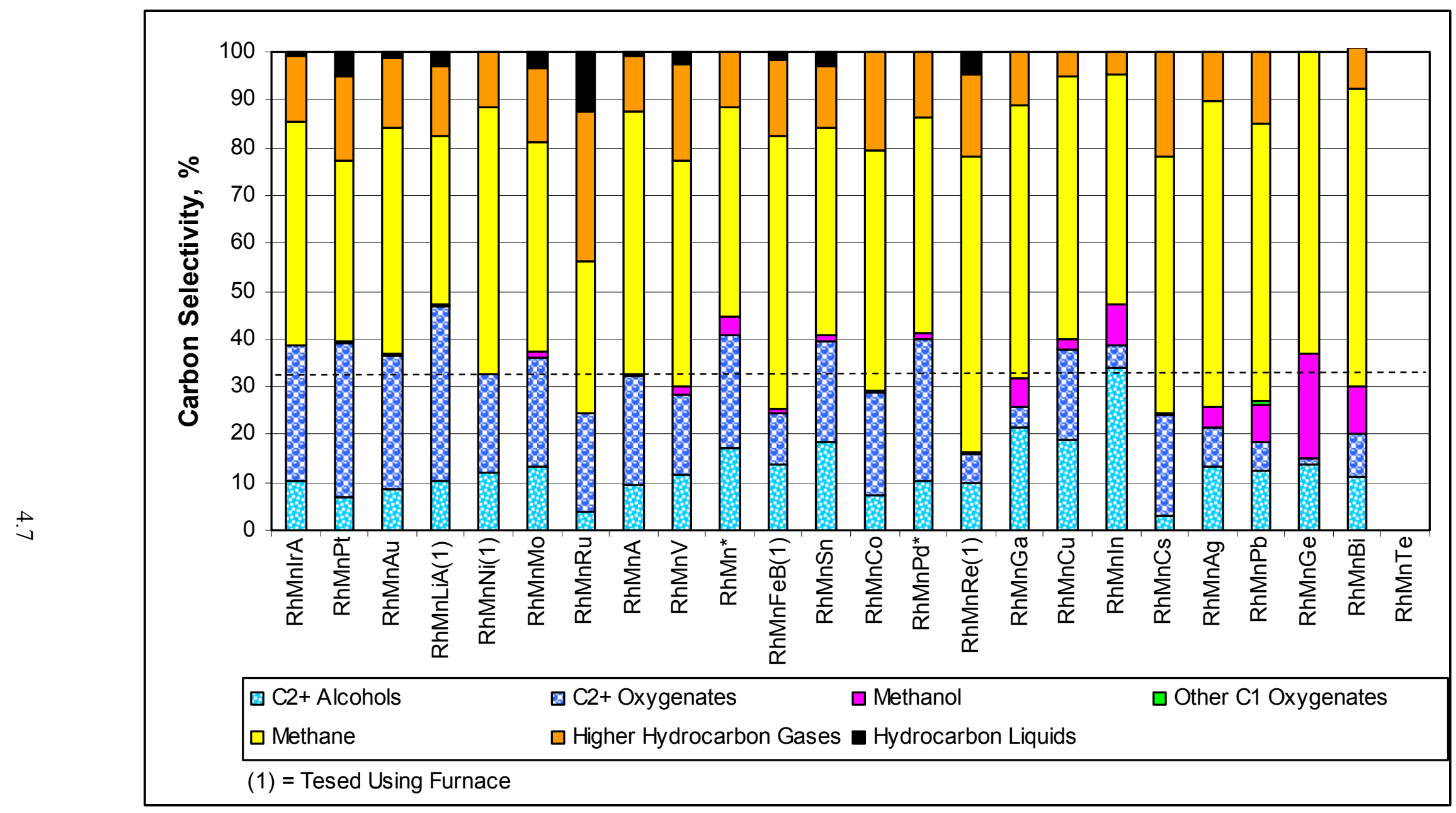

Figure 4.2. Carbon Selectivities of Promoted Catalysts at Conditions for Maximum STYs 
Table 4.3. Comparison of Carbon Conversions and STYs of Promoted $\mathrm{RhMn} / \mathrm{SiO}_{2}$ Catalysts at $300^{\circ} \mathrm{C}, 11,000 \mathrm{~L} / \mathrm{L}_{\text {cat }} / \mathrm{hr}$

\begin{tabular}{|c|c|c|c|c|c|c|c|c|c|c|c|}
\hline \multirow[b]{2}{*}{ Catalyst } & \multirow[b]{2}{*}{$\begin{array}{c}\text { Space } \\
\text { Velocity, } \\
\text { L/L/hr }\end{array}$} & \multirow[b]{2}{*}{$\begin{array}{c}\text { Temp., } \\
{ }^{\circ} \mathrm{C}\end{array}$} & \multirow[b]{2}{*}{$\begin{array}{c}\text { Carbon } \\
\text { Conv., } \\
\%\end{array}$} & \multirow[b]{2}{*}{$\begin{array}{c}\mathrm{CO} \\
\text { Conv., } \\
\%\end{array}$} & \multirow[b]{2}{*}{$\begin{array}{c}\text { CO Conv. } \\
\text { to } \mathrm{CO}_{2} \\
\%\end{array}$} & \multicolumn{5}{|c|}{$\mathrm{STY}, \mathrm{g} / \mathrm{mL}_{\mathrm{cat}} / \mathrm{hr}$} & \multirow{2}{*}{$\begin{array}{c}\text { Carbon } \\
\text { Balance } \\
\left(\mathrm{C}_{\text {out }} / \mathrm{C}_{\text {in }}\right), \\
\% \%\end{array}$} \\
\hline & & & & & & $\mathrm{MEOH}$ & $\begin{array}{c}\mathrm{C}_{2}+ \\
\text { Alcohols } \\
\end{array}$ & $\begin{array}{c}\text { Other } \mathrm{C}_{2}+ \\
\text { Oxygenates }\end{array}$ & $\begin{array}{c}\mathrm{HC} \\
\text { Liquids }\end{array}$ & $\begin{array}{c}\text { Total } \\
\text { Liquids }\end{array}$ & \\
\hline RhMnIrA & 11,000 & 303 & 36.6 & 40.3 & -1.10 & 0.000 & 0.106 & 0.541 & 0.012 & 0.659 & 94.79 \\
\hline $\mathrm{RhMnPt}$ & 11,000 & 300 & 30.8 & 34.7 & 0.07 & 0.003 & 0.061 & 0.432 & 0.042 & 0.538 & 97.57 \\
\hline RhMnAu & 11,000 & 300 & 27.3 & 30.5 & -0.48 & 0.005 & 0.088 & 0.400 & 0.030 & 0.523 & 98.19 \\
\hline $\operatorname{RhMnLiA}^{(\mathrm{a})}$ & 11,000 & 305 & 24.5 & 28.2 & 0.67 & 0.006 & 0.099 & 0.386 & 0.019 & 0.510 & 97.13 \\
\hline RhMnMo & 12,000 & 298 & 30.1 & 38.9 & 4.95 & 0.016 & 0.165 & 0.306 & 0.027 & 0.514 & 98.49 \\
\hline $\operatorname{RhMnLiA}^{(a)}$ & 11,000 & 300 & 19.6 & 21.8 & -0.15 & 0.007 & 0.092 & 0.357 & 0.008 & 0.464 & 97.92 \\
\hline RhMnRu & 11,000 & 300 & 43.4 & 49.6 & 0.26 & 0.000 & 0.069 & 0.372 & 0.139 & 0.581 & 102.25 \\
\hline RhMnA & 11,000 & 300 & 23.4 & 26.4 & 0.11 & 0.006 & 0.086 & 0.330 & 0.035 & 0.457 & 96.27 \\
\hline $\mathrm{RhMnLi}^{(\mathrm{a})}$ & 11,000 & 302 & 18.9 & 21.1 & -0.33 & 0.007 & 0.086 & 0.325 & 0.031 & 0.449 & 98.45 \\
\hline RhMnV & 11,000 & 299 & 31.5 & 37.1 & 1.35 & 0.026 & 0.148 & 0.222 & 0.022 & 0.418 & 102.36 \\
\hline RhMn* & 11,000 & 299 & 21.4 & 24.1 & -0.12 & 0.046 & 0.149 & 0.214 & 0.000 & 0.409 & 96.33 \\
\hline RhMnCo & 11,000 & 299 & 28.5 & 31.7 & -0.18 & 0.000 & 0.075 & 0.276 & 0.055 & 0.406 & 94.65 \\
\hline $\mathrm{RhMnFeB}^{(\mathrm{a})}$ & 11,000 & 300 & 27.5 & 32.1 & 0.10 & 0.008 & 0.128 & 0.204 & 0.015 & 0.356 & 97.89 \\
\hline RhMnPd* & 12,000 & 300 & 16.7 & 18.2 & -0.56 & 0.009 & 0.072 & 0.228 & 0.000 & 0.310 & 96.99 \\
\hline $\mathrm{RhMnNi}^{\text {(a) }}$ & 11,000 & 300 & 18.0 & 19.7 & -0.52 & 0.000 & 0.065 & 0.202 & 0.001 & 0.269 & 91.48 \\
\hline RhMnSn & 11,000 & 299 & 11.7 & 13.8 & 0.59 & 0.007 & 0.082 & 0.152 & 0.012 & 0.252 & 105.41 \\
\hline $\mathrm{RhMnCu}$ & 11,000 & 300 & 6.4 & 6.9 & -0.26 & 0.006 & 0.037 & 0.059 & 0.000 & 0.101 & 97.23 \\
\hline RhMnGa & 11,000 & 300 & 5.3 & 4.9 & -1.19 & 0.042 & 0.079 & 0.016 & 0.000 & 0.137 & 97.52 \\
\hline RhMnCs & 11,000 & 301 & 5.5 & 6.4 & -0.44 & 0.003 & 0.009 & 0.085 & 0.000 & 0.097 & 99.00 \\
\hline RhMnAg & 11,000 & 300 & 5.8 & 7.6 & 1.03 & 0.026 & 0.037 & 0.030 & 0.000 & 0.094 & 104.85 \\
\hline RhMnIn & 11,000 & 300 & 3.0 & 3.7 & 0.28 & 0.018 & 0.035 & 0.006 & 0.000 & 0.059 & 101.15 \\
\hline IrMn & 11,000 & 302 & 1.5 & 0.9 & -0.86 & 0.064 & 0.005 & 0.000 & 0.000 & 0.069 & 96.47 \\
\hline RhMnGe & 11,000 & 301 & 0.9 & 0.6 & -0.47 & 0.008 & 0.004 & 0.000 & 0.000 & 0.012 & 94.39 \\
\hline $\mathrm{RhMnPb}$ & 11,000 & 300 & 1.4 & 2.2 & 0.59 & 0.002 & 0.002 & 0.002 & 0.000 & 0.006 & 103.41 \\
\hline $\mathrm{RhMnBi}$ & 11,000 & 301 & 0 & 0 & -0.45 & 0 & 0 & 0 & 0 & 0 & 98.87 \\
\hline RhMnTe & 11,000 & 300 & 0 & 0 & 0.91 & 0 & 0 & 0 & 0 & 0 & 106.90 \\
\hline
\end{tabular}




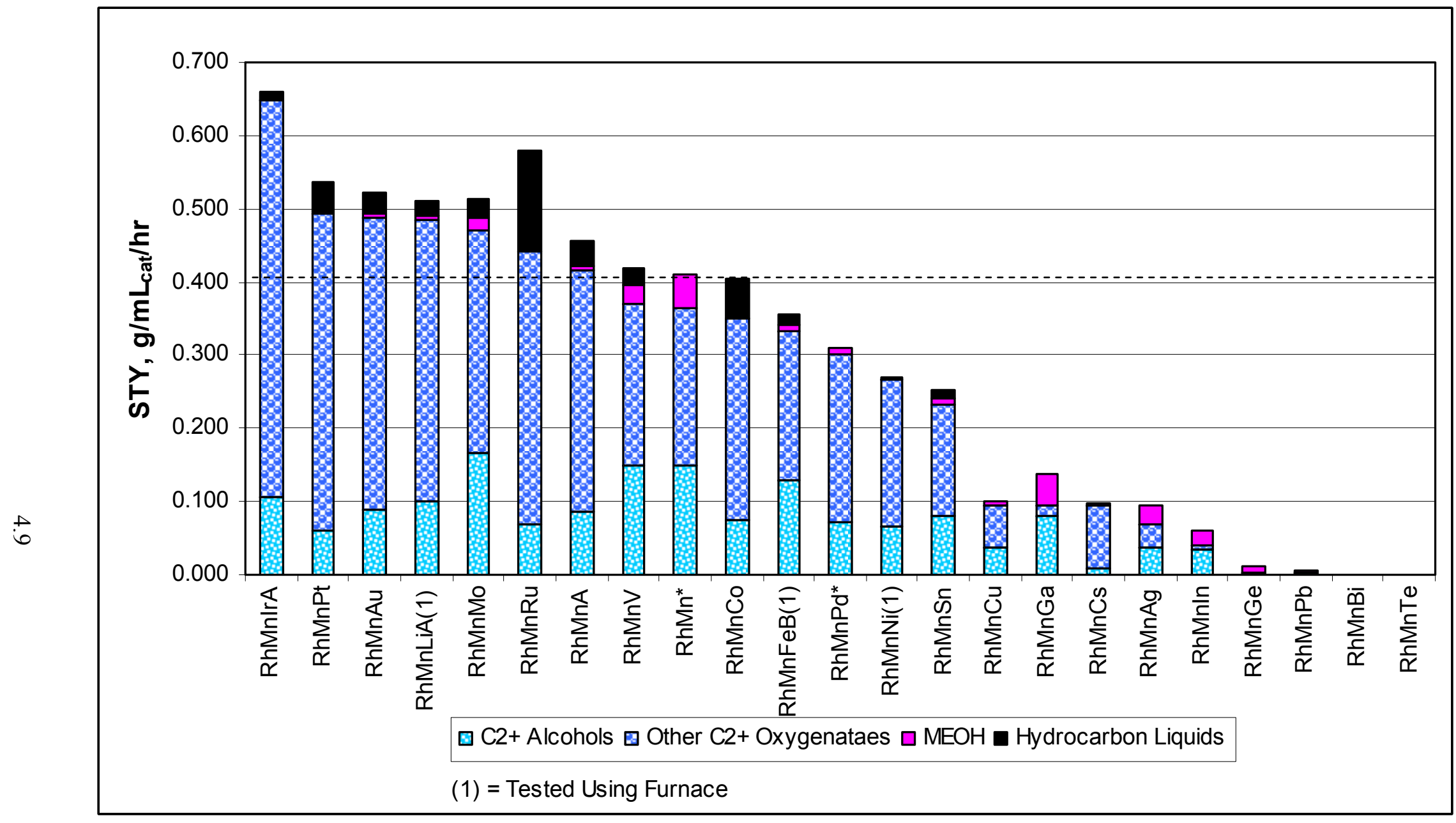

Figure 4.3. STYs of Promoted Catalysts at $300^{\circ} \mathrm{C}, 11,000 \mathrm{~L} / \mathrm{Lcat} / \mathrm{hr}$ 
Table 4.4. Comparison of Carbon Selectivities to Products of Promoted $\mathrm{RhMn} / \mathrm{SiO}_{2}$ Catalysts at $300^{\circ} \mathrm{C}, 11000 \mathrm{~L} / \mathrm{L}_{\mathrm{cat}} / \mathrm{hr}$

\begin{tabular}{|c|c|c|c|c|c|c|c|c|c|c|}
\hline \multirow[b]{2}{*}{ Catalyst } & \multirow{2}{*}{$\begin{array}{c}\text { Space } \\
\text { Velocity, } \\
\text { L/L/hr }\end{array}$} & \multirow[b]{2}{*}{$\begin{array}{l}\text { Tem., } \\
{ }^{\circ} \mathrm{C}\end{array}$} & \multirow{2}{*}{$\begin{array}{c}\text { Carbon } \\
\text { Conv., } \\
\%\end{array}$} & \multicolumn{4}{|c|}{ Carbon Selectivity (C-Mol\%) } & \multirow[b]{2}{*}{ Methane } & \multirow[b]{2}{*}{$\begin{array}{l}\text { Higher HC } \\
\text { Gases }\end{array}$} & \multirow[b]{2}{*}{$\begin{array}{l}\text { Higher HC } \\
\text { Liquids (est) }\end{array}$} \\
\hline & & & & $\mathrm{MEOH}$ & $\begin{array}{c}\text { Other } \mathrm{C}_{1} \\
\text { Oxygenates }\end{array}$ & $\begin{array}{c}\mathrm{C}_{2}+ \\
\text { Alcohols }\end{array}$ & $\begin{array}{c}\text { Other } \mathrm{C}_{2}+ \\
\text { Oxygenates }\end{array}$ & & & \\
\hline RhMnIrA & 11,000 & 303 & 36.6 & 0.00 & 0.00 & 7.26 & 34.58 & 37.80 & 19.13 & 1.22 \\
\hline $\mathrm{RhMnPt}$ & 11,000 & 300 & 30.8 & 0.18 & 0.00 & 5.06 & 32.96 & 36.57 & 19.89 & 5.33 \\
\hline $\mathrm{RhMnAu}$ & 11,000 & 300 & 27.3 & 0.35 & 0.00 & 8.09 & 34.16 & 38.21 & 14.90 & 4.29 \\
\hline $\operatorname{RhMnLiA}^{(a)}$ & 11,000 & 305 & 24.5 & 0.42 & 0.00 & 10.32 & 36.55 & 34.90 & 14.66 & 3.14 \\
\hline RhMnMo & 12,000 & 298 & 30.1 & 0.88 & 0.00 & 13.45 & 22.79 & 44.18 & 15.31 & 3.38 \\
\hline $\operatorname{RhMnLiA}^{(\mathrm{a})}$ & 11,000 & 300 & 19.6 & 0.65 & 0.00 & 12.01 & 41.33 & 29.36 & 14.96 & 1.68 \\
\hline RhMnRu & 11,000 & 300 & 43.4 & 0.00 & 0.00 & 4.04 & 20.29 & 32.06 & 31.25 & 12.37 \\
\hline RhMnA & 11,000 & 300 & 23.4 & 0.41 & 0.00 & 9.10 & 32.31 & 39.21 & 13.25 & 5.71 \\
\hline $\mathrm{RhMnLi}^{(\mathrm{a})}$ & 11,000 & 302 & 18.9 & 0.66 & 0.00 & 11.41 & 38.38 & 26.70 & 16.61 & 6.24 \\
\hline RhMnV & 11,000 & 299 & 31.5 & 1.42 & 0.00 & 11.77 & 16.73 & 47.47 & 19.91 & 2.70 \\
\hline RhMn* & 11,000 & 299 & 21.4 & 3.72 & 0.00 & 17.29 & 23.54 & 43.76 & 11.69 & 0.00 \\
\hline RhMnCo & 11,000 & 299 & 28.5 & 0.00 & 0.00 & 6.76 & 23.70 & 43.84 & 18.00 & 7.70 \\
\hline $\mathrm{RhMnFeB}^{(\mathrm{a})}$ & 11,000 & 300 & 27.5 & 0.60 & 0.00 & 13.19 & 19.88 & 49.94 & 13.92 & 2.46 \\
\hline $\mathrm{RhMnPd}^{*}$ & 12,000 & 300 & 16.7 & 0.93 & 0.00 & 10.34 & 29.73 & 45.44 & 13.56 & 0.00 \\
\hline $\mathrm{RhMnNi}^{(\mathrm{a})}$ & 11,000 & 300 & 18.0 & 0.00 & 0.00 & 8.59 & 24.17 & 51.32 & 15.62 & 0.29 \\
\hline RhMnSn & 11,000 & 299 & 11.7 & 1.04 & 0.00 & 17.55 & 29.51 & 37.29 & 10.75 & 3.86 \\
\hline $\mathrm{RhMnCu}$ & 11,000 & 300 & 6.4 & 1.59 & 0.00 & 14.31 & 21.28 & 59.03 & 3.79 & 0.00 \\
\hline $\mathrm{RhMnGa}$ & 11,000 & 300 & 5.3 & 13.71 & 0.00 & 36.80 & 6.67 & 42.07 & 0.76 & 0.00 \\
\hline RhMnCs & 11,000 & 301 & 5.5 & 0.88 & 0.00 & 4.36 & 33.35 & 43.57 & 18.51 & 0.00 \\
\hline RhMnAg & 11,000 & 300 & 5.8 & 8.08 & 0.00 & 16.30 & 12.80 & 62.82 & 0.00 & 0.00 \\
\hline RhMnIn & 11,000 & 300 & 3.0 & 10.14 & 0.00 & 29.36 & 4.06 & 29.61 & 26.99 & 0.00 \\
\hline IrMn & 11,000 & 302 & 1.5 & 73.46 & 0.00 & 7.76 & 0.47 & 18.32 & 0.00 & 0.00 \\
\hline $\mathrm{RhMnGe}$ & 11,000 & 301 & 0.9 & 16.37 & 0.00 & 10.34 & 0.70 & 56.62 & 15.96 & 0.00 \\
\hline $\mathrm{RhMnPb}$ & 11,000 & 300 & 1.4 & 2.79 & 0.02 & 3.57 & 2.48 & 22.28 & 69.01 & 0.00 \\
\hline RhMnBi & 11,000 & 301 & NA & NA & NA & NA & NA & NA & NA & NA \\
\hline RhMnTe & 11,000 & 300 & NA & NA & NA & NA & NA & NA & NA & NA \\
\hline
\end{tabular}




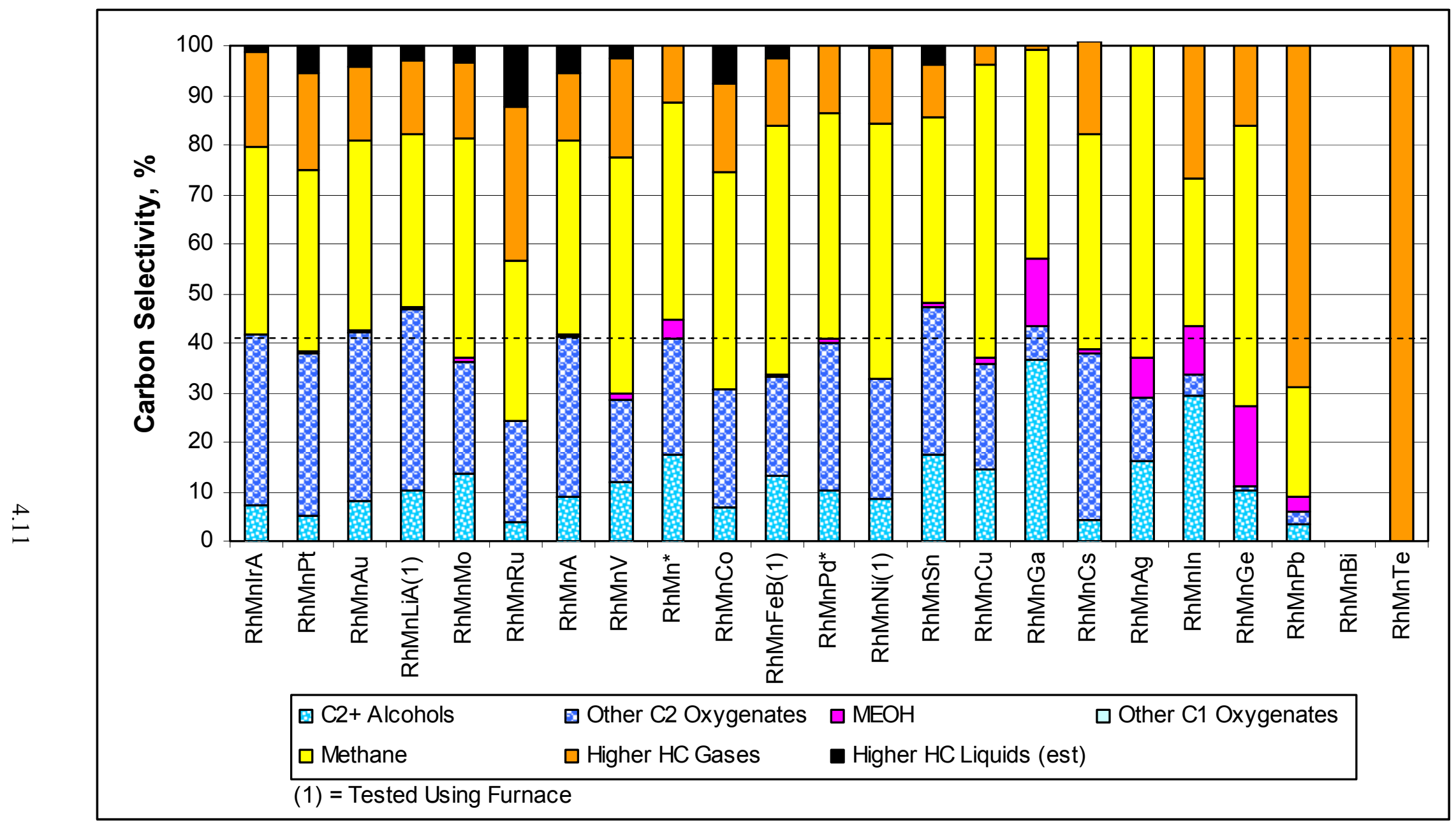

Figure 4.4. Carbon Selectivities of Promoted Catalysts at $300^{\circ} \mathrm{C}, 11,000 \mathrm{~L} / \mathrm{L}_{\mathrm{cat}} / \mathrm{hr}$ 
None of the catalysts having maximum $\mathrm{C}_{2}+$-oxygenate $\mathrm{STYs}$ greater than the baseline $\mathrm{Rh}-\mathrm{Mn} / \mathrm{SiO}_{2}$ catalyst had very good selectivity to $\mathrm{C}_{2}+$ alcohols. However, among the catalysts that were less active than the $\mathrm{RhMn} / \mathrm{SiO}_{2}$ catalyst at both the conditions of maximum STYs and at the common testing conditions $\left(300^{\circ} \mathrm{C}, 11,000 \mathrm{~L} / \mathrm{L}_{\mathrm{cat}} / \mathrm{hr}\right)$, the Ga- and In-promoted catalysts have achieved the high selectivity of the $\mathrm{C}_{2}+$ oxygenates to $\mathrm{C}_{2}+$ alcohols. For example, at the conditions of highest STYs (Table 4.2 and Figure 4.2), ethanol accounted for 83 and $87 \%$ of the total $\mathrm{C}_{2}+$ oxygenates for the Ga- and In-promoted catalysts, respectively. However, STYs under these conditions were 0.188 , and $0.138 \mathrm{~L} / \mathrm{L}_{\text {cat }} / \mathrm{hr}$, respectively, which is well below that of the baseline catalyst. These catalysts also produced significant quantities of methanol, although this may be attributable to variations of the $\mathrm{SiO}_{2}$ support as previously discussed. 


\subsection{Conclusions and Recommendations}

Based on the results of the tests performed, several promoters (i.e., Ir, Pt, $\mathrm{Au}, \mathrm{Li}, \mathrm{Ni}, \mathrm{Mo}$, and $\mathrm{Ru}$ ) achieved $\mathrm{C}_{2}+$-oxygenate STYs that were as good or better than the best unpromoted $\mathrm{RhMn} / \mathrm{SiO}_{2}$ catalyst tested. Of these, the Ir-promoted catalyst is the best, achieving a nearly $80 \%$ improvement over the unpromoted catalyst, while the Mo- and Ru-promoted catalysts showed clear evidence of unacceptable deactivation.

While none of the catalysts tested have very good selectivity to $\mathrm{C}_{2}+$ oxygenates, the Li-promoted catalyst had the best selectivity achieving approximately $47 \%$ carbon selectivity to oxygenates at conditions where it achieved its highest $\mathrm{C}_{2}+$-oxygenates STY, which was approximately $45 \%$ better than the unpromoted catalyst. The catalysts promoted by $\mathrm{Ir}, \mathrm{Pt}$, and $\mathrm{Au}$ also showed better carbon selectivity to $\mathrm{C}_{2}+$ oxygenates than the unpromoted catalysts, but not as much as the Ir-promoted catalyst.

None of the more active catalysts had a very good selectivity of all oxygenates to $\mathrm{C}_{2}+$ alcohols. However, among the less active catalysts, the Ga- and In-promoted catalysts had demonstrated very good selectivities of oxygenates to $\mathrm{C}_{2}+$ alcohols (approximately $70 \%$ ) at their respective conditions for maximum $\mathrm{C}_{2}+$-oxygenate STYs. Further research is needed to address the selectivity of the more promising catalysts either by the addition of a fourth component on the catalyst to improve hydrogenation of the nonalcoholic oxygenates or by use of a separate catalyst that would hydrogenate the mixed oxygenates product to alcohols. It also is recommended that test conditions favoring oxygenate selectivity and catalyst reactivity be investigated to improve the STY and yield of $\mathrm{C}_{2}+$ oxygenates. These conditions would include higher pressures and lower $\mathrm{H}_{2}$ :CO ratios in the feed gas.

Further testing is needed to evaluate the more promising promoters to determine the optimum ratios and concentrations of $\mathrm{Rh}, \mathrm{Mn}$ and promoters on the $\mathrm{SiO}_{2}$ support. Furthermore, we found that the source of $\mathrm{SiO}_{2}$ could have a significant effect on the STYs and carbon selectivities. This also suggests that other supports could have similar effects on catalyst performance. These effects need to be further investigated.

All of the catalysts tested showed deactivation to varying degrees when the catalyst temperature was above $300^{\circ} \mathrm{C}$. Further research with the more promising catalysts will be needed to assess whether deactivation can be stabilized at acceptable levels at the temperatures needed to obtain acceptable $\mathrm{C}_{2}+-$ oxygenate STYs. 



\subsection{References}

Gerber MA, JF White, and DJ Stevens. 2007. Mixed Alcohol Synthesis Catalyst Screening. PNNL-16763, Pacific Northwest National Laboratory, Richland, Washington.

Tsotsis TT, VUS Rao, and LM Polinski. 1982. "Reaction Rate Oscillations during Fischer\} Tropsch Synthesis on Fe-Precipitated Nu-1 Zeolite-Type Catalysts." A.I.Ch.E. Journal 28:847-851.

Phillips S, A Aden, J Jechura, D Dayton, and T Eggeman. 2007. Thermochemical Ethanol via Indirect Gasification and Mixed Alcohol Synthesis of Lignocellulosic Biomass. NREL-TP-510-41168, National Renewable Energy Laboratory, Golden, Colorado.

Quarderer GJ. 1986. "Mixed Alcohols from Synthesis Gas." In Proceedings from the $78^{\text {th }}$ Spring National AIChE Meeting, April 1986, New Orleans, Louisiana.

Quarderer GJ and GA Cochran. 1986. Process for Producing Alcohols from Synthesis Gas. U.S. Patent No. 4,749,724.

Stiles AB, F Chen, JB Harrison, X Hu, DA Storm, and HX Yang. 1991. "Catalytic Conversion of Synthesis Gas to Methanol and Other Oxygenated Products." Industrial and Engineering Chemistry Research 30:811-821. 

Appendix A

\section{Catalyst Testing Data}





\section{Appendix A}

\section{Catalyst Testing Data}

Table A.1. Summary of Test Results

\begin{tabular}{|c|c|c|c|c|c|c|c|c|c|c|c|c|c|c|c|c|c|c|}
\hline \multirow[b]{2}{*}{ Catalyst } & \multirow[b]{2}{*}{$\begin{array}{c}\text { Space } \\
\text { Velocity } \\
\left(\mathrm{L} / \mathrm{L}_{\text {cat }} h r\right)\end{array}$} & \multirow[b]{2}{*}{$\begin{array}{l}\text { Temp. } \\
\left({ }^{\circ} \mathrm{C}\right)\end{array}$} & \multirow[b]{2}{*}{$\begin{array}{l}\text { Carbon } \\
\text { Conv. } \\
(\%)\end{array}$} & \multirow[b]{2}{*}{$\begin{array}{l}\text { CO } \\
\text { Conv. } \\
(\%)\end{array}$} & \multirow{2}{*}{$\begin{array}{c}\mathrm{CO} \\
\text { Conv. } \\
\text { to } \mathrm{CO}_{2} \\
(\%) \\
\end{array}$} & \multicolumn{6}{|c|}{ Carbon Selectivity (C-Mol\%) } & \multicolumn{6}{|c|}{ STY $\left(\mathrm{g} / \mathrm{mL}_{\mathrm{cat}} / \mathrm{hr}\right)$} & \multirow{2}{*}{$\begin{array}{c}\text { Carbon } \\
\text { Balance } \\
\left(\mathrm{C}_{\text {out }} / \mathrm{C}_{\text {in }}\right) \\
\% \\
\%\end{array}$} \\
\hline & & & & & & $\mathrm{MeOH}$ & $\begin{array}{c}\text { Other } \mathrm{C}_{1} \\
\text { Oxygenates }\end{array}$ & $\begin{array}{l}\mathrm{C}_{2}+ \\
\text { Alc. }\end{array}$ & $\begin{array}{c}\text { Other } \mathrm{C}_{2}+ \\
\text { Oxygenates }\end{array}$ & $\mathrm{CH}_{4}$ & $\begin{array}{l}\text { Other } \\
\text { HCs } \\
\text { (est) } \\
\end{array}$ & $\begin{array}{l}\mathrm{C}_{2}+ \\
\text { Alc. }\end{array}$ & $\begin{array}{c}\text { Other } \mathrm{C}_{2}+ \\
\text { Oxygenates }\end{array}$ & $\begin{array}{c}\text { Total } \mathrm{C}_{2}+ \\
\text { Oxygenates } \\
\end{array}$ & $\begin{array}{c}\mathrm{HC} \\
\text { Liquids }\end{array}$ & $\mathrm{MeOH}$ & $\begin{array}{l}\text { Total } \\
\text { Liq. }\end{array}$ & \\
\hline \multirow{11}{*}{$\mathrm{RhMn} / \mathrm{SiO}_{2} \mathrm{~A}^{\mathrm{c}}$} & 7,500 & 256 & 6.16 & 6.67 & -0.26 & 0.48 & 0.01 & 7.85 & 30.46 & 36.98 & 24.22 & 0.01 & 0.06 & 0.07 & 0.00 & 0.00 & 0.08 & 95.9 \\
\hline & 7,500 & 277 & 18.25 & 20.78 & 0.25 & 0.50 & 0.00 & 8.35 & 43.24 & 31.71 & 16.21 & 0.04 & 0.23 & 0.28 & 0.01 & 0.00 & 0.29 & 101.0 \\
\hline & 11,000 & 300 & 23.38 & 26.37 & 0.11 & 0.41 & 0.00 & 9.10 & 32.31 & 39.21 & 18.96 & 0.09 & 0.33 & 0.42 & 0.03 & 0.01 & 0.46 & 96.3 \\
\hline & 11,000 & 317 & 36.77 & 41.50 & 0.17 & 0.37 & 0.00 & 8.66 & 25.84 & 46.71 & 18.41 & 0.13 & 0.31 & 0.44 & 0.02 & 0.01 & 0.46 & 98.9 \\
\hline & 11,000 & 314 & 27.96 & 31.56 & 0.08 & 0.34 & 0.00 & 9.12 & 22.59 & 53.65 & 14.30 & 0.10 & 0.33 & 0.43 & 0.01 & 0.01 & 0.45 & 94.4 \\
\hline & 11,000 & 324 & 32.57 & 37.24 & 0.60 & 0.34 & 0.00 & 9.25 & 22.90 & 55.19 & 12.32 & 0.12 & 0.32 & 0.44 & 0.01 & 0.01 & 0.45 & 98.1 \\
\hline & 15,000 & 324 & 23.17 & 26.36 & 0.23 & 0.51 & 0.00 & 9.41 & 19.41 & 59.76 & 10.91 & 0.12 & 0.26 & 0.37 & 0.00 & 0.01 & 0.38 & 96.3 \\
\hline & 15,000 & 315 & 20.66 & 23.09 & -0.25 & 0.68 & 0.00 & 9.67 & 25.01 & 52.16 & 12.48 & 0.11 & 0.29 & 0.40 & 0.00 & 0.01 & 0.41 & 97.9 \\
\hline & 15,000 & 302 & 12.06 & 13.32 & -0.29 & 0.85 & 0.00 & 9.40 & 25.87 & 52.93 & 10.95 & 0.06 & 0.18 & 0.24 & 0.00 & 0.01 & 0.24 & 97.5 \\
\hline & 11,000 & 302 & 18.23 & 20.54 & 0.00 & 0.87 & 0.00 & 10.16 & 29.49 & 47.06 & 12.41 & 0.07 & 0.23 & 0.31 & 0.00 & 0.01 & 0.31 & 98.5 \\
\hline & 7,500 & 256 & 2.30 & 2.62 & 0.03 & 1.50 & 0.00 & 12.42 & 21.86 & 61.09 & 3.12 & 0.01 & 0.02 & 0.02 & 0.00 & 0.00 & 0.02 & 98.3 \\
\hline \multirow{6}{*}{$\mathrm{RhMn} / \mathrm{SiO}_{2} *$} & 75,00 & 255 & 6.86 & 7.31 & -0.49 & 2.34 & 0.00 & 18.38 & 41.41 & 34.82 & 3.06 & 0.03 & 0.08 & 0.12 & 0.00 & 0.01 & 0.12 & 97.8 \\
\hline & 7,500 & 276 & 15.27 & 17.06 & -0.22 & 1.92 & 0.00 & 16.59 & 27.70 & 39.36 & 14.43 & 0.07 & 0.12 & 0.19 & 0.00 & 0.01 & 0.20 & 94.4 \\
\hline & 11,000 & 299 & 21.42 & 24.14 & -0.12 & 3.72 & 0.00 & 17.29 & 23.54 & 43.76 & 11.69 & 0.15 & 0.21 & 0.36 & 0.00 & 0.05 & 0.41 & 96.3 \\
\hline & 11,000 & 314 & 24.52 & 28.33 & 0.52 & 5.04 & 0.00 & 16.33 & 13.89 & 54.07 & 10.66 & 0.16 & 0.14 & 0.30 & 0.00 & 0.07 & 0.38 & 97.0 \\
\hline & 11,000 & 315 & 23.28 & 27.42 & 1.12 & 6.45 & 0.00 & 14.97 & 11.73 & 56.25 & 10.60 & 0.14 & 0.11 & 0.26 & 0.00 & 0.09 & 0.34 & 98.6 \\
\hline & 7,500 & 275 & 4.21 & 4.96 & 0.20 & 6.96 & 0.00 & 15.72 & 21.66 & 54.70 & 0.96 & 0.02 & 0.03 & 0.05 & 0.00 & 0.01 & 0.06 & 100.2 \\
\hline \multirow{5}{*}{$\begin{array}{l}\mathrm{Rh} / \mathrm{Mn} / \mathrm{Fe} / \mathrm{SiO}_{2}{ }^{(\mathrm{a}, \mathrm{b}, \mathrm{c})} \\
\left(\mathrm{H}_{2}: \mathrm{CO}=2.5-2.6\right)\end{array}$} & 7,400 & 257 & 21.75 & 26.21 & 0.41 & 0.24 & 0.08 & 14.24 & 24.79 & 37.17 & 23.48 & 0.07 & 0.14 & 0.21 & 0.00 & 0.00 & 0.21 & 97.9 \\
\hline & 15,000 & 257 & 9.43 & 11.23 & -0.01 & 0.43 & 0.08 & 12.19 & 24.19 & 36.74 & 26.36 & 0.05 & 0.12 & 0.17 & 0.00 & 0.00 & 0.17 & 98.9 \\
\hline & 7,400 & 285 & 36.34 & 43.12 & -0.35 & 0.17 & 0.08 & 13.55 & 19.92 & 47.56 & 18.72 & 0.11 & 0.18 & 0.30 & 0.00 & 0.00 & 0.30 & 90.9 \\
\hline & 11,000 & 323 & 46.92 & 56.34 & 0.01 & 0.31 & 0.13 & 16.58 & 7.36 & 58.87 & 16.76 & 0.27 & 0.13 & 0.40 & 0.00 & 0.01 & 0.41 & 92.2 \\
\hline & 11,000 & 326 & 45.37 & 55.54 & 0.40 & 0.38 & 0.00 & 15.56 & 5.52 & 63.62 & 14.91 & 0.25 & 0.09 & 0.34 & 0.00 & 0.01 & 0.35 & 95.4 \\
\hline \multirow{4}{*}{$\begin{array}{l}\mathrm{Rh} / \mathrm{Mn} / \mathrm{Fe} / \mathrm{SiO}_{2}{ }^{(\mathrm{a}, \mathrm{b}, \mathrm{c})}(\mathrm{H} \\
\left.{ }_{2}: \mathrm{CO}=2.0\right) \\
\mathrm{Rh} / \mathrm{Mn} / \mathrm{Fe} / \mathrm{SiO}_{2}{ }^{(\mathrm{a}, \mathrm{b}, \mathrm{c})} \\
\left(\mathrm{H}_{2}: \mathrm{CO}=2.4\right)\end{array}$} & 11,000 & 326 & 38.96 & 45.51 & 1.92 & 0.33 & 0.00 & 15.04 & 8.08 & 59.06 & 17.49 & 0.22 & 0.13 & 0.35 & 0.00 & 0.01 & 0.36 & 96.5 \\
\hline & 15,000 & 326 & 32.00 & 37.21 & 1.57 & 0.47 & 0.08 & 14.22 & 7.76 & 61.79 & 15.69 & 0.23 & 0.14 & 0.37 & 0.00 & 0.01 & 0.38 & 97.0 \\
\hline & 15,000 & 354 & 26.82 & 32.87 & 1.73 & 1.00 & 0.00 & 8.76 & 2.91 & 82.90 & 4.43 & 0.11 & 0.04 & 0.15 & 0.00 & 0.02 & 0.17 & 97.8 \\
\hline & 15,000 & 402 & 65.05 & 90.11 & 14.85 & 0.04 & 0.00 & 0.32 & 0.10 & 97.78 & 1.75 & 0.01 & 0.00 & 0.01 & 0.00 & 0.00 & 0.01 & 93.1 \\
\hline \multirow{3}{*}{$\begin{array}{l}\mathrm{RhMnFe} / \mathrm{SiO}_{2} \mathrm{~B}^{(\mathrm{b}, \mathrm{c})} \\
\left(\mathrm{H}_{2}: \mathrm{CO}=2.4\right)\end{array}$} & 7,500 & 255 & 17.09 & 18.02 & -1.71 & 0.54 & 0.00 & 14.29 & 24.09 & 33.28 & 27.80 & 0.06 & 0.11 & 0.17 & 0.02 & 0.00 & 0.19 & 94.0 \\
\hline & 7,500 & 275 & 29.69 & 32.06 & -2.40 & 0.24 & 0.00 & 11.56 & 18.82 & 36.56 & 32.82 & 0.08 & 0.14 & 0.22 & 0.07 & 0.00 & 0.30 & 90.3 \\
\hline & 11,000 & 327 & 42.38 & 50.47 & 1.51 & 0.54 & 0.00 & 14.67 & 5.51 & 63.10 & 16.17 & 0.22 & 0.09 & 0.32 & 0.02 & 0.01 & 0.35 & 95.6 \\
\hline \multirow{4}{*}{$\begin{array}{l}\mathrm{RhMnFe} / \mathrm{SiO}_{2} \mathrm{~B}^{(\mathrm{b}, \mathrm{c})} \\
\left(\mathrm{H}_{2}: \mathrm{CO}=1.9\right) \\
\mathrm{RhMnFe} / \mathrm{SiO}_{2} \mathrm{~B}^{(\mathrm{b}, \mathrm{c})} \\
\left(\mathrm{H}_{2}: \mathrm{CO}=2.4\right)\end{array}$} & 11,000 & 325 & 35.29 & 41.82 & 1.55 & 0.53 & 0.00 & 13.66 & 10.97 & 57.16 & 17.67 & 0.20 & 0.17 & 0.36 & 0.01 & 0.01 & 0.39 & 97.7 \\
\hline & 11,000 & 300 & 23.70 & 26.46 & -0.17 & 0.50 & 0.00 & 11.53 & 23.10 & 45.58 & 19.29 & 0.11 & 0.24 & 0.35 & 0.01 & 0.01 & 0.37 & 96.2 \\
\hline & 11,000 & 300 & 27.49 & 32.05 & 0.10 & 0.60 & 0.00 & 13.19 & 19.88 & 49.94 & 16.38 & 0.13 & 0.20 & 0.33 & 0.02 & 0.01 & 0.36 & 97.9 \\
\hline & 7,500 & 275 & 21.35 & 23.96 & -0.65 & 0.68 & 0.00 & 13.38 & 29.05 & 40.78 & 16.12 & 0.07 & 0.16 & 0.22 & 0.01 & 0.00 & 0.23 & 93.1 \\
\hline
\end{tabular}


Table A.1. (contd)

\begin{tabular}{|c|c|c|c|c|c|c|c|c|c|c|c|c|c|c|c|c|c|c|}
\hline \multirow[b]{2}{*}{ Catalyst } & \multirow[b]{2}{*}{$\begin{array}{c}\text { Space } \\
\text { Velocity } \\
\left(\mathrm{L} / \mathrm{L}_{\text {cat }} \mathrm{hr}\right)\end{array}$} & \multirow[b]{2}{*}{$\begin{array}{l}\text { Temp. } \\
\left({ }^{\circ} \mathrm{C}\right)\end{array}$} & \multirow[b]{2}{*}{$\begin{array}{l}\text { Carbon } \\
\text { Conv. } \\
(\%)\end{array}$} & \multirow[b]{2}{*}{$\begin{array}{c}\text { CO } \\
\text { Conv. } \\
(\%)\end{array}$} & \multirow{2}{*}{$\begin{array}{c}\mathrm{CO} \\
\text { Conv. } \\
\text { to } \mathrm{CO}_{2} \\
(\%)\end{array}$} & \multicolumn{6}{|c|}{ Carbon Selectivity (C-Mol\%) } & \multicolumn{6}{|c|}{$\mathrm{STY}\left(\mathrm{g} / \mathrm{mL}_{\mathrm{cat}} / \mathrm{hr}\right)$} & \multirow{2}{*}{$\begin{array}{c}\text { Carbon } \\
\text { Balance } \\
\left(\mathrm{C}_{\text {out }} / \mathrm{C}_{\text {in }}\right) \\
\%\end{array}$} \\
\hline & & & & & & $\mathrm{MeOH}$ & $\begin{array}{c}\text { Other } \mathrm{C}_{1} \\
\text { Oxygenates }\end{array}$ & $\begin{array}{l}\mathrm{C}_{2}+ \\
\text { Alc. }\end{array}$ & $\begin{array}{c}\text { Other } \mathrm{C}_{2}+ \\
\text { Oxygenates }\end{array}$ & $\mathrm{CH}_{4}$ & $\begin{array}{l}\text { Other } \\
\text { HCs } \\
\text { (est) }\end{array}$ & $\begin{array}{l}\mathrm{C}_{2}+ \\
\text { Alc. }\end{array}$ & $\begin{array}{c}\text { Other } \mathrm{C}_{2}+ \\
\text { Oxygenates }\end{array}$ & $\begin{array}{c}\text { Total } \mathrm{C}_{2}+ \\
\text { Oxygenates } \\
\end{array}$ & $\begin{array}{c}\mathrm{HC} \\
\text { Liquids }\end{array}$ & $\mathrm{MeOH}$ & $\begin{array}{l}\text { Total } \\
\text { Liq. }\end{array}$ & \\
\hline \multirow{5}{*}{$\mathrm{RhMnFe} / \mathrm{SiO}_{2} \mathrm{~A}^{(\mathrm{b}, \mathrm{c})}$} & 7,500 & 255 & 17.53 & 18.45 & -1.88 & 0.31 & 0.00 & 14.12 & 24.45 & 32.15 & 28.97 & 0.06 & 0.12 & 0.18 & 0.02 & 0.00 & 0.20 & 96.1 \\
\hline & 7,500 & 275 & 28.84 & 31.82 & -1.27 & 0.31 & 0.00 & 13.01 & 23.18 & 32.90 & 30.60 & 0.09 & 0.18 & 0.27 & 0.05 & 0.00 & 0.32 & 92.8 \\
\hline & 11,000 & 325 & 38.10 & 44.77 & 1.62 & 0.24 & 0.00 & 12.70 & 8.95 & 58.15 & 19.97 & 0.20 & 0.15 & 0.35 & 0.01 & 0.01 & 0.36 & 93.2 \\
\hline & 11,000 & 325 & 35.61 & 41.85 & 1.35 & 0.30 & 0.00 & 14.57 & 8.69 & 54.73 & 21.71 & 0.21 & 0.14 & 0.35 & 0.03 & 0.01 & 0.39 & 95.2 \\
\hline & 9,300 & 301 & 29.41 & 34.59 & 1.11 & 0.36 & 0.00 & 12.27 & 18.44 & 47.44 & 21.49 & 0.12 & 0.20 & 0.32 & 0.01 & 0.01 & 0.34 & 94.7 \\
\hline \multirow{7}{*}{$\mathrm{RhMnLi} / \mathrm{SiO}_{2}{ }^{(\mathrm{b}, \mathrm{c})}$} & 7,500 & 256 & 4.69 & 4.08 & -1.24 & 0.93 & 0.00 & 13.16 & 44.36 & 25.19 & 16.35 & 0.02 & 0.07 & 0.08 & 0.00 & 0.00 & 0.09 & 95.3 \\
\hline & 7,500 & 277 & 10.88 & 10.93 & -1.36 & 0.80 & 0.00 & 10.78 & 38.18 & 28.21 & 22.03 & 0.03 & 0.13 & 0.16 & 0.00 & 0.00 & 0.16 & 92.0 \\
\hline & 11,000 & 302 & 17.98 & 19.44 & -0.88 & 0.66 & 0.00 & 11.38 & 39.35 & 29.36 & 19.26 & 0.08 & 0.31 & 0.39 & 0.00 & 0.01 & 0.40 & 93.9 \\
\hline & 11,000 & 302 & 18.92 & 21.06 & -0.33 & 0.66 & 0.00 & 11.41 & 38.38 & 26.70 & 22.85 & 0.09 & 0.32 & 0.41 & 0.03 & 0.01 & 0.45 & 98.5 \\
\hline & 11,000 & 293 & 2.40 & 2.30 & -0.42 & 2.61 & 0.00 & 17.30 & 33.68 & 47.00 & 0.00 & 0.02 & 0.04 & 0.05 & 0.00 & 0.00 & 0.06 & 98.0 \\
\hline & 11,000 & 326 & 8.13 & 9.97 & 0.74 & 2.12 & 0.00 & 17.14 & 17.24 & 52.10 & 11.77 & 0.06 & 0.07 & 0.12 & 0.00 & 0.01 & 0.13 & 95.3 \\
\hline & 11,000 & 350 & 23.88 & 30.27 & 2.93 & 1.79 & 0.00 & 20.33 & 15.91 & 47.91 & 14.39 & 0.20 & 0.18 & 0.37 & 0.00 & 0.02 & 0.40 & 98.7 \\
\hline \multirow{4}{*}{$\mathrm{RhMnLi} / \mathrm{SiO}_{2} \mathrm{~A}^{(\mathrm{b}, \mathrm{c})}$} & 7,500 & 257 & 4.74 & 5.34 & 0.03 & 1.82 & 0.00 & 11.87 & 50.36 & 25.26 & 10.69 & 0.02 & 0.08 & 0.09 & 0.00 & 0.00 & 0.10 & 97.0 \\
\hline & 7,500 & 277 & 11.14 & 12.47 & -0.05 & 0.65 & 0.00 & 9.68 & 40.98 & 27.92 & 20.77 & 0.03 & 0.14 & 0.17 & 0.00 & 0.00 & 0.17 & 93.5 \\
\hline & 11,000 & 300 & 19.56 & 21.84 & -0.15 & 0.65 & 0.00 & 12.01 & 41.33 & 29.36 & 16.64 & 0.09 & 0.36 & 0.45 & 0.01 & 0.01 & 0.46 & 97.9 \\
\hline & 11,000 & 305 & 24.49 & 28.21 & 0.67 & 0.42 & 0.00 & 10.32 & 36.55 & 34.90 & 17.81 & 0.10 & 0.39 & 0.48 & 0.02 & 0.01 & 0.51 & 97.1 \\
\hline \multirow{3}{*}{$\mathrm{RhMnIr} / \mathrm{SiO}_{2}{ }^{(\mathrm{b}, \mathrm{c})}$} & 7,500 & 256 & 21.47 & 24.07 & -0.05 & 0.00 & 0.00 & 5.47 & 52.18 & 22.12 & 20.47 & 0.03 & 0.34 & 0.37 & 0.00 & 0.00 & 0.37 & 95.9 \\
\hline & 7,500 & 267 & 29.07 & 31.92 & -0.70 & 0.00 & 0.00 & 6.42 & 36.77 & 29.58 & 27.24 & 0.05 & 0.32 & 0.37 & 0.03 & 0.00 & 0.40 & 89.9 \\
\hline & 11,000 & 328 & 50.78 & 58.25 & 1.32 & 0.14 & 0.00 & 8.53 & 14.40 & 55.01 & 21.92 & 0.18 & 0.32 & 0.50 & 0.02 & 0.00 & 0.52 & 94.3 \\
\hline \multirow{8}{*}{$\mathrm{RhMnIr} / \mathrm{SiO}_{2} \mathrm{~A}^{(\mathrm{c})}$} & 7,500 & 256 & 17.70 & 21.73 & 1.93 & 0.00 & 0.00 & 6.57 & 46.72 & 26.03 & 20.69 & 0.03 & 0.26 & 0.29 & 0.00 & 0.00 & 0.29 & 110.5 \\
\hline & 7,500 & 276 & 30.87 & 34.66 & 0.14 & 0.00 & 0.00 & 6.57 & 41.22 & 28.83 & 23.38 & 0.06 & 0.39 & 0.44 & 0.02 & 0.00 & 0.46 & 94.2 \\
\hline & 11,000 & 303 & 36.63 & 40.26 & -1.10 & 0.00 & 0.00 & 7.26 & 34.58 & 37.80 & 20.36 & 0.11 & 0.54 & 0.65 & 0.01 & 0.00 & 0.66 & 94.8 \\
\hline & 11,000 & 316 & 43.18 & 48.80 & 0.54 & 0.00 & 0.00 & 9.26 & 29.01 & 40.49 & 21.25 & 0.16 & 0.53 & 0.69 & 0.04 & 0.00 & 0.72 & 97.5 \\
\hline & 11,000 & 323 & 43.58 & 49.72 & 0.97 & 0.00 & 0.00 & 9.69 & 23.16 & 47.39 & 19.76 & 0.17 & 0.43 & 0.60 & 0.03 & 0.00 & 0.62 & 95.0 \\
\hline & 15,000 & 325 & 37.79 & 42.72 & 0.42 & 0.25 & 0.00 & 10.25 & 28.32 & 46.66 & 14.52 & 0.21 & 0.60 & 0.81 & 0.01 & 0.01 & 0.83 & 99.4 \\
\hline & 11,000 & 303 & 28.92 & 32.34 & -0.11 & 0.00 & 0.00 & 8.39 & 33.66 & 39.08 & 18.87 & 0.10 & 0.41 & 0.51 & 0.00 & 0.00 & 0.51 & 95.5 \\
\hline & 11,000 & 304 & 27.14 & 30.23 & -0.44 & 0.00 & 0.00 & 8.70 & 34.14 & 40.96 & 16.20 & 0.09 & 0.39 & 0.48 & 0.00 & 0.00 & 0.48 & 95.7 \\
\hline \multirow{7}{*}{$\mathrm{RhMnNi} / \mathrm{SiO}_{2}{ }^{(\mathrm{b}, \mathrm{c})}$} & 7,500 & 256 & 7.74 & 8.17 & -0.58 & 0.00 & 0.00 & 9.71 & 46.05 & 33.41 & 10.83 & 0.02 & 0.11 & 0.13 & 0.00 & 0.00 & 0.13 & 96.5 \\
\hline & 7,500 & 277 & 16.67 & 18.44 & -0.27 & 0.00 & 0.00 & 7.94 & 37.72 & 36.85 & 17.48 & 0.04 & 0.19 & 0.23 & 0.00 & 0.00 & 0.23 & 93.1 \\
\hline & 11,000 & 300 & 18.00 & 19.66 & -0.52 & 0.00 & 0.00 & 8.59 & 24.17 & 51.32 & 15.91 & 0.06 & 0.20 & 0.27 & 0.00 & 0.00 & 0.27 & 91.5 \\
\hline & 11,000 & 305 & 18.25 & 19.96 & -0.48 & 0.00 & 0.00 & 11.11 & 28.57 & 46.16 & 14.16 & 0.09 & 0.24 & 0.32 & 0.00 & 0.00 & 0.32 & 93.9 \\
\hline & 11,000 & 325 & 34.06 & 39.05 & 1.00 & 0.00 & 0.00 & 11.86 & 20.60 & 56.10 & 11.44 & 0.17 & 0.31 & 0.48 & 0.00 & 0.00 & 0.48 & 99.8 \\
\hline & 7,500 & 312 & 36.91 & 42.42 & 1.08 & 0.00 & 0.00 & 11.33 & 26.08 & 49.15 & 13.45 & 0.12 & 0.28 & 0.40 & 0.01 & 0.00 & 0.40 & 95.9 \\
\hline & 7,500 & 277 & 11.99 & 12.90 & -0.61 & 0.00 & 0.01 & 13.40 & 37.34 & 36.45 & 12.80 & 0.04 & 0.13 & 0.18 & 0.00 & 0.00 & 0.18 & 96.9 \\
\hline \multirow{4}{*}{$\mathrm{RhMnRe} / \mathrm{SiO}_{2}{ }^{(\mathrm{b}, \mathrm{c})}$} & 7,500 & 256 & 19.02 & 21.33 & -0.06 & 0.00 & 0.00 & 9.07 & 27.29 & 31.45 & 32.19 & 0.05 & 0.16 & 0.21 & 0.03 & 0.00 & 0.24 & 98.1 \\
\hline & 7,500 & 273 & 28.97 & 32.34 & -0.23 & 0.00 & 0.00 & 8.35 & 19.34 & 35.55 & 36.76 & 0.07 & 0.17 & 0.24 & 0.07 & 0.00 & 0.30 & 95.2 \\
\hline & 7,500 & 273 & 27.57 & 30.98 & 0.13 & 0.00 & 0.00 & 10.39 & 18.09 & 36.61 & 34.90 & 0.08 & 0.15 & 0.22 & 0.06 & 0.00 & 0.28 & 91.4 \\
\hline & 11,000 & 324 & 37.98 & 44.82 & 1.63 & 0.40 & 0.00 & 9.72 & 6.09 & 62.07 & 21.71 & 0.15 & 0.10 & 0.26 & 0.05 & 0.01 & 0.31 & 94.6 \\
\hline \multirow{3}{*}{$\mathrm{RhMnCu} / \mathrm{SiO}_{2}$} & 7,500 & 256 & 1.10 & 1.54 & 0.32 & 0.00 & 0.00 & 0.00 & 0.00 & 95.96 & 4.04 & 0.00 & 0.00 & 0.00 & 0.00 & 0.00 & 0.00 & 96.8 \\
\hline & 7,500 & 276 & 4.95 & 5.90 & 0.40 & 1.60 & 0.00 & 15.63 & 30.66 & 46.99 & 5.11 & 0.02 & 0.04 & 0.07 & 0.00 & 0.003 & 0.07 & 98.8 \\
\hline & 11,000 & 300 & 6.41 & 6.91 & -0.26 & 1.59 & 0.00 & 14.31 & 21.28 & 59.03 & 3.79 & 0.04 & 0.06 & 0.10 & 0.00 & 0.01 & 0.10 & 97.2 \\
\hline
\end{tabular}


Table A.1. (contd)

\begin{tabular}{|c|c|c|c|c|c|c|c|c|c|c|c|c|c|c|c|c|c|c|}
\hline \multirow[b]{2}{*}{ Catalyst } & \multirow[b]{2}{*}{$\begin{array}{c}\text { Space } \\
\text { Velocity } \\
\left(\mathrm{L} / \mathrm{L}_{\text {cat }} \mathrm{hr}\right) \\
\end{array}$} & \multirow[b]{2}{*}{$\begin{array}{l}\text { Temp. } \\
\left({ }^{\circ} \mathrm{C}\right)\end{array}$} & \multirow[b]{2}{*}{$\begin{array}{c}\text { Carbon } \\
\text { Conv. } \\
(\%)\end{array}$} & \multirow[b]{2}{*}{$\begin{array}{c}\text { CO } \\
\text { Conv. } \\
(\%)\end{array}$} & \multirow{2}{*}{$\begin{array}{c}\mathrm{CO} \\
\text { Conv. } \\
\text { to } \mathrm{CO}_{2} \\
(\%) \\
\end{array}$} & \multicolumn{6}{|c|}{ Carbon Selectivity (C-Mol\%) } & \multicolumn{6}{|c|}{ STY $\left(\mathrm{g} / \mathrm{mL}_{\text {cat }} / \mathrm{hr}\right)$} & \multirow{2}{*}{$\begin{array}{c}\text { Carbon } \\
\text { Balance } \\
\left(\mathrm{C}_{\text {out }} / \mathrm{C}_{\text {in }}\right) \\
\% \\
\%\end{array}$} \\
\hline & & & & & & $\mathrm{MeOH}$ & $\begin{array}{c}\text { Other } \mathrm{C}_{1} \\
\text { Oxygenates }\end{array}$ & $\begin{array}{l}\mathrm{C}_{2}+ \\
\text { Alc. }\end{array}$ & $\begin{array}{l}\text { Other } \mathrm{C}_{2}+ \\
\text { Oxygenates }\end{array}$ & $\mathrm{CH}_{4}$ & $\begin{array}{l}\text { Other } \\
\text { HCs } \\
\text { (est) }\end{array}$ & $\begin{array}{l}\mathrm{C}_{2}+ \\
\text { Alc. }\end{array}$ & $\begin{array}{c}\text { Other } \mathrm{C}_{2}+ \\
\text { Oxygenates }\end{array}$ & $\begin{array}{c}\text { Total } \mathrm{C}_{2}+ \\
\text { Oxygenates }\end{array}$ & $\begin{array}{c}\mathrm{HC} \\
\text { Liquids }\end{array}$ & $\mathrm{MeOH}$ & $\begin{array}{l}\text { Total } \\
\text { Liq. }\end{array}$ & \\
\hline & 11,000 & 315 & 9.46 & 10.37 & -0.23 & 2.19 & 0.00 & 18.76 & 18.97 & 54.82 & 5.26 & 0.07 & 0.08 & 0.15 & 0.00 & 0.01 & 0.16 & 98.0 \\
\hline & 11,000 & 324 & 9.47 & 10.43 & -0.19 & 2.06 & 0.00 & 15.11 & 11.35 & 63.71 & 7.78 & 0.06 & 0.05 & 0.10 & 0.00 & 0.01 & 0.11 & 96.9 \\
\hline & 11,000 & 337 & 12.14 & 13.26 & -0.36 & 2.25 & 0.00 & 13.14 & 8.08 & 68.56 & 7.97 & 0.06 & 0.04 & 0.11 & 0.00 & 0.02 & 0.12 & 95.4 \\
\hline \multirow{6}{*}{$\mathrm{RhMnCo} / \mathrm{SiO}_{2}$} & 7,500 & 256 & 9.19 & 10.56 & 0.31 & 0.77 & 0.00 & 7.76 & 33.03 & 33.74 & 24.70 & 0.02 & 0.09 & 0.11 & 0.00 & 0.00 & 0.11 & 95.4 \\
\hline & 7,500 & 256 & 9.93 & 10.05 & -1.18 & 0.95 & 0.00 & 9.60 & 40.85 & 29.20 & 19.40 & 0.03 & 0.12 & 0.14 & 0.00 & 0.00 & 0.15 & 96.8 \\
\hline & 7,500 & 277 & 21.91 & 24.35 & -0.14 & 0.00 & 0.00 & 6.85 & 30.73 & 32.45 & 29.96 & 0.04 & 0.19 & 0.23 & 0.03 & 0.00 & 0.26 & 95.2 \\
\hline & 11,000 & 299 & 28.48 & 31.69 & -0.18 & 0.00 & 0.00 & 6.76 & 23.70 & 43.84 & 25.70 & 0.08 & 0.28 & 0.35 & 0.06 & 0.00 & 0.41 & 94.7 \\
\hline & 11,000 & 323 & 37.57 & 42.94 & 0.94 & 0.48 & 0.00 & 9.68 & 11.30 & 60.62 & 17.92 & 0.14 & 0.18 & 0.32 & 0.03 & 0.01 & 0.36 & 95.3 \\
\hline & 11,000 & 307 & 29.37 & 33.08 & 0.02 & 0.33 & 0.00 & 7.25 & 21.65 & 50.20 & 20.57 & 0.08 & 0.27 & 0.35 & 0.00 & 0.01 & 0.36 & 95.4 \\
\hline \multirow{7}{*}{$\mathrm{RhMnMo} / \mathrm{SiO}_{2}$} & 7,500 & 256 & 21.22 & 24.89 & 1.07 & 1.78 & 0.00 & 20.46 & 22.76 & 32.11 & 22.88 & 0.11 & 0.14 & 0.26 & 0.03 & 0.01 & 0.30 & 97.9 \\
\hline & 7,500 & 275 & 33.52 & 41.92 & 4.25 & 1.15 & 0.00 & 17.76 & 14.58 & 36.99 & 29.52 & 0.16 & 0.15 & 0.31 & 0.07 & 0.01 & 0.39 & 93.0 \\
\hline & 11,000 & 298 & 30.06 & 38.95 & 4.95 & 0.88 & 0.00 & 13.45 & 22.79 & 44.18 & 18.69 & 0.17 & 0.31 & 0.47 & 0.03 & 0.02 & 0.51 & 98.5 \\
\hline & 11,000 & 314 & 30.60 & 41.62 & 7.30 & 1.25 & 0.00 & 15.28 & 14.78 & 54.99 & 13.71 & 0.20 & 0.20 & 0.40 & 0.01 & 0.02 & 0.43 & 96.3 \\
\hline & 11,000 & 325 & 30.53 & 44.75 & 10.38 & 1.56 & 0.00 & 3.56 & 8.18 & 66.97 & 19.73 & 0.04 & 0.11 & 0.16 & 0.00 & 0.03 & 0.19 & 92.9 \\
\hline & 15,000 & 324 & 25.86 & 34.63 & 5.55 & 2.04 & 0.00 & 13.31 & 9.58 & 59.33 & 15.74 & 0.20 & 0.15 & 0.35 & 0.00 & 0.04 & 0.39 & 98.5 \\
\hline & 15,000 & 316 & 21.06 & 27.28 & 3.58 & 2.13 & 0.00 & 13.16 & 10.78 & 57.25 & 16.67 & 0.16 & 0.14 & 0.30 & 0.00 & 0.04 & 0.33 & 97.7 \\
\hline \multirow{6}{*}{$\mathrm{RhMnPd} / \mathrm{SiO}_{2}$} & 7,500 & 256 & 6.08 & 7.12 & 0.29 & 0.83 & 0.02 & 7.14 & 42.61 & 37.77 & 11.64 & 0.01 & 0.08 & 0.10 & 0.00 & 0.00 & 0.10 & 97.9 \\
\hline & 7,500 & 275 & 12.62 & 13.97 & -0.25 & 0.52 & 0.01 & 6.74 & 35.16 & 38.21 & 19.37 & 0.02 & 0.14 & 0.16 & 0.01 & 0.00 & 0.17 & 98.8 \\
\hline & 11,000 & 300 & 16.66 & 18.18 & -0.56 & 0.93 & 0.00 & 10.34 & 29.73 & 45.44 & 13.56 & 0.07 & 0.23 & 0.30 & 0.00 & 0.01 & 0.31 & 97.0 \\
\hline & 11,000 & 323 & 21.14 & 24.03 & 0.24 & 1.05 & 0.00 & 9.99 & 15.44 & 60.20 & 13.32 & 0.09 & 0.15 & 0.24 & 0.00 & 0.01 & 0.25 & 99.2 \\
\hline & 11,000 & 314 & 17.14 & 19.73 & 0.50 & 1.31 & 0.00 & 9.33 & 15.81 & 52.50 & 21.05 & 0.07 & 0.13 & 0.19 & 0.00 & 0.01 & 0.21 & 102.0 \\
\hline & 11,000 & 302 & 11.18 & 12.73 & 0.15 & 0.63 & 0.01 & 4.25 & 14.74 & 59.75 & 20.61 & 0.02 & 0.08 & 0.10 & 0.00 & 0.00 & 0.10 & 99.3 \\
\hline \multirow{7}{*}{$\mathrm{RhMnPt} / \mathrm{SiO}_{2}$} & 7,500 & 255 & 13.00 & 14.51 & -0.14 & 0.23 & 0.01 & 5.42 & 45.92 & 24.71 & 23.71 & 0.02 & 0.18 & 0.20 & 0.00 & 0.00 & 0.20 & 99.8 \\
\hline & 7,500 & 275 & 26.50 & 29.28 & -0.52 & 0.18 & 0.01 & 4.72 & 39.44 & 27.74 & 27.91 & 0.03 & 0.30 & 0.33 & 0.03 & 0.00 & 0.36 & 96.0 \\
\hline & 11,000 & 306 & 32.59 & 36.57 & -0.11 & 0.16 & 0.00 & 5.38 & 30.61 & 37.17 & 26.69 & 0.07 & 0.43 & 0.50 & 0.03 & 0.00 & 0.53 & 96.9 \\
\hline & 11,000 & 300 & 30.83 & 34.65 & 0.07 & 0.18 & 0.00 & 5.06 & 32.96 & 36.57 & 25.22 & 0.06 & 0.43 & 0.49 & 0.04 & 0.00 & 0.54 & 97.6 \\
\hline & 11,000 & 313 & 40.32 & 45.32 & -0.05 & 0.14 & 0.00 & 6.74 & 32.41 & 37.93 & 22.78 & 0.11 & 0.56 & 0.66 & 0.05 & 0.00 & 0.72 & 99.0 \\
\hline & 11,000 & 307 & 28.28 & 31.60 & -0.29 & 0.26 & 0.00 & 6.02 & 29.56 & 43.46 & 20.70 & 0.07 & 0.36 & 0.43 & 0.01 & 0.00 & 0.44 & 96.3 \\
\hline & 15,000 & 315 & 25.21 & 28.29 & -0.13 & 0.29 & 0.00 & 6.66 & 31.30 & 41.31 & 20.43 & 0.09 & 0.45 & 0.54 & 0.03 & 0.01 & 0.57 & 98.9 \\
\hline \multirow{8}{*}{$\mathrm{RhMnRu} / \mathrm{SiO}_{2}$} & 7,500 & 256 & 19.28 & 22.02 & 0.25 & 0.00 & 0.01 & 3.43 & 32.46 & 19.63 & 44.47 & 0.02 & 0.18 & 0.20 & 0.03 & 0.00 & 0.22 & 99.9 \\
\hline & 7,500 & 274 & 35.81 & 40.37 & -0.06 & 0.00 & 0.00 & 2.89 & 21.60 & 21.41 & 54.10 & 0.03 & 0.22 & 0.25 & 0.11 & 0.00 & 0.36 & 98.1 \\
\hline & 11,000 & 300 & 43.38 & 49.61 & 0.26 & 0.00 & 0.00 & 4.04 & 20.29 & 32.06 & 43.62 & 0.07 & 0.37 & 0.44 & 0.14 & 0.00 & 0.58 & 102.3 \\
\hline & 11,000 & 313 & 44.09 & 50.60 & 0.55 & 0.10 & 0.00 & 5.10 & 17.41 & 41.13 & 36.26 & 0.09 & 0.32 & 0.41 & 0.07 & 0.00 & 0.49 & 101.2 \\
\hline & 11,000 & 323 & 38.33 & 44.78 & 1.40 & 0.29 & 0.00 & 6.63 & 16.33 & 49.32 & 27.44 & 0.10 & 0.26 & 0.36 & 0.02 & 0.01 & 0.39 & 101.2 \\
\hline & 15,000 & 325 & 25.83 & 29.48 & 0.20 & 0.39 & 0.00 & 6.10 & 13.52 & 55.35 & 24.65 & 0.08 & 0.19 & 0.28 & 0.00 & 0.01 & 0.29 & 98.7 \\
\hline & 15,000 & 303 & 15.26 & 17.17 & -0.11 & 0.56 & 0.00 & 6.44 & 22.86 & 46.42 & 23.72 & 0.05 & 0.20 & 0.25 & 0.00 & 0.01 & 0.25 & 97.7 \\
\hline & 11,000 & 302 & 23.25 & 26.62 & 0.34 & 0.39 & 0.00 & 6.41 & 19.79 & 42.36 & 31.06 & 0.06 & 0.19 & 0.25 & 0.01 & 0.01 & 0.27 & 97.8 \\
\hline \multirow{4}{*}{$\mathrm{RhMnGa} / \mathrm{SiO}_{2}$} & 7,500 & 255 & 0.97 & 0.69 & -0.41 & 9.23 & 0.00 & 38.07 & 7.57 & 43.48 & 1.66 & 0.01 & 0.00 & 0.01 & 0.00 & 0.00 & 0.02 & 99.6 \\
\hline & 7,500 & 275 & 3.55 & 4.27 & 0.27 & 9.94 & 0.00 & 33.06 & 5.62 & 30.99 & 20.38 & 0.03 & 0.01 & 0.04 & 0.00 & 0.01 & 0.05 & 99.1 \\
\hline & 11,000 & 300 & 5.31 & 4.85 & -1.19 & 13.71 & 0.00 & 36.80 & 6.67 & 42.07 & 0.76 & 0.08 & 0.02 & 0.09 & 0.00 & 0.04 & 0.14 & 97.5 \\
\hline & 11,000 & 324 & 12.59 & 13.92 & -0.34 & 7.91 & 0.00 & 27.58 & 4.93 & 43.68 & 15.89 & 0.14 & 0.03 & 0.17 & 0.00 & 0.06 & 0.23 & 98.5 \\
\hline
\end{tabular}


Table A.1. (contd)

\begin{tabular}{|c|c|c|c|c|c|c|c|c|c|c|c|c|c|c|c|c|c|c|}
\hline \multirow[b]{2}{*}{ Catalyst } & \multirow[b]{2}{*}{$\begin{array}{c}\text { Space } \\
\text { Velocity } \\
\left(\mathrm{L} / \mathrm{L}_{\text {cat }} \mathrm{hr}\right)\end{array}$} & \multirow[b]{2}{*}{$\begin{array}{l}\text { Temp. } \\
\left({ }^{\circ} \mathrm{C}\right)\end{array}$} & \multirow[b]{2}{*}{$\begin{array}{c}\text { Carbon } \\
\text { Conv. } \\
(\%) \\
\end{array}$} & \multirow[b]{2}{*}{$\begin{array}{c}\text { CO } \\
\text { Conv. } \\
(\%)\end{array}$} & \multirow{2}{*}{$\begin{array}{c}\mathrm{CO} \\
\text { Conv. } \\
\text { to } \mathrm{CO}_{2} \\
(\%) \\
\end{array}$} & \multicolumn{6}{|c|}{ Carbon Selectivity (C-Mol\%) } & \multicolumn{6}{|c|}{ STY $\left(\mathrm{g} / \mathrm{mL}_{\text {cat }} / \mathrm{hr}\right)$} & \multirow{2}{*}{$\begin{array}{c}\text { Carbon } \\
\text { Balance } \\
\left(\mathrm{C}_{\text {out }} / \mathrm{C}_{\text {in }}\right) \\
\% \\
\end{array}$} \\
\hline & & & & & & $\mathrm{MeOH}$ & $\begin{array}{c}\text { Other } \mathrm{C}_{1} \\
\text { Oxygenates }\end{array}$ & $\begin{array}{l}\mathrm{C}_{2}+ \\
\text { Alc. }\end{array}$ & $\begin{array}{c}\text { Other } \mathrm{C}_{2}+ \\
\text { Oxyggenates }\end{array}$ & $\mathrm{CH}_{4}$ & $\begin{array}{l}\text { Other } \\
\mathrm{HCs} \\
\text { (est) }\end{array}$ & $\begin{array}{l}\mathrm{C}_{2}+ \\
\text { Alc. }\end{array}$ & $\begin{array}{c}\text { Other } \mathrm{C}_{2}+ \\
\text { Oxygenates }\end{array}$ & $\begin{array}{c}\text { Total } \mathrm{C}_{2}+ \\
\text { Oxygenates } \\
\end{array}$ & $\begin{array}{c}\mathrm{HC} \\
\text { Liquids }\end{array}$ & $\mathrm{MeOH}$ & $\begin{array}{l}\text { Total } \\
\text { Liq. }\end{array}$ & \\
\hline & 11,000 & 345 & 17.77 & 20.91 & 0.83 & 6.27 & 0.00 & 21.38 & 4.24 & 57.03 & 11.08 & 0.15 & 0.03 & 0.19 & 0.00 & 0.06 & 0.25 & 99.3 \\
\hline \multirow{5}{*}{$\mathrm{RhMnIn} / \mathrm{SiO}_{2}$} & 7,500 & 256 & 0.95 & 1.15 & 0.05 & 7.19 & 0.03 & 33.77 & 31.92 & 0.00 & 28.97 & 0.01 & 0.01 & 0.02 & 0.00 & 0.00 & 0.02 & 100.6 \\
\hline & 7,500 & 276 & 1.04 & 0.19 & -0.99 & 13.90 & 0.00 & 35.74 & 3.97 & 46.59 & 0.00 & 0.01 & 0.00 & 0.01 & 0.00 & 0.01 & 0.02 & 97.6 \\
\hline & 11,000 & 300 & 3.00 & 3.68 & 0.28 & 10.14 & 0.00 & 29.36 & 4.06 & 29.61 & 26.99 & 0.04 & 0.01 & 0.04 & 0.00 & 0.02 & 0.06 & 101.2 \\
\hline & 11,000 & 325 & 4.66 & 5.46 & 0.17 & 10.04 & 0.00 & 37.71 & 4.10 & 46.14 & 2.01 & 0.07 & 0.01 & 0.08 & 0.00 & 0.03 & 0.11 & 99.1 \\
\hline & 11,000 & 345 & 8.71 & 10.17 & 0.30 & 8.45 & 0.00 & 33.84 & 4.94 & 47.95 & 4.81 & 0.12 & 0.02 & 0.14 & 0.00 & 0.04 & 0.18 & 98.5 \\
\hline \multirow{7}{*}{$\mathrm{RhMnSn} / \mathrm{SiO}_{2}$} & 7,500 & 256 & 2.67 & 3.09 & 0.08 & 2.39 & 0.02 & 21.83 & 37.01 & 37.73 & 1.02 & 0.02 & 0.03 & 0.05 & 0.00 & 0.00 & 0.05 & 99.5 \\
\hline & 7,500 & 276 & 7.86 & 9.65 & 0.81 & 0.75 & 0.00 & 13.57 & 20.45 & 35.75 & 29.47 & 0.03 & 0.05 & 0.07 & 0.01 & 0.00 & 0.09 & 105.7 \\
\hline & 11,000 & 299 & 11.75 & 13.84 & 0.59 & 1.04 & 0.00 & 17.55 & 29.51 & 37.29 & 14.61 & 0.08 & 0.15 & 0.23 & 0.01 & 0.01 & 0.25 & 105.4 \\
\hline & 11,000 & 314 & 16.93 & 19.11 & 0.14 & 0.94 & 0.00 & 17.21 & 21.59 & 42.59 & 17.66 & 0.12 & 0.16 & 0.27 & 0.02 & 0.01 & 0.30 & 98.3 \\
\hline & 11,000 & 325 & 21.58 & 23.84 & -0.51 & 1.07 & 0.00 & 18.44 & 21.07 & 43.60 & 15.82 & 0.16 & 0.20 & 0.35 & 0.02 & 0.01 & 0.38 & 99.0 \\
\hline & 15,000 & 325 & 14.87 & 16.44 & -0.36 & 1.23 & 0.00 & 17.59 & 19.00 & 45.96 & 16.22 & 0.14 & 0.16 & 0.30 & 0.02 & 0.01 & 0.33 & 98.5 \\
\hline & 11,000 & 342 & 24.06 & 27.46 & 0.33 & 1.27 & 0.00 & 19.16 & 15.39 & 49.97 & 14.21 & 0.18 & 0.16 & 0.35 & 0.02 & 0.02 & 0.38 & 101.2 \\
\hline \multirow{4}{*}{$\mathrm{RhMnGe} / \mathrm{SiO}_{2}$} & 7,500 & 256 & 0.04 & 0.88 & 0.83 & 33.04 & 0.00 & 18.47 & 2.73 & 0.00 & 45.76 & 0.00 & 0.00 & 0.00 & 0.00 & 0.00 & 0.00 & 106.4 \\
\hline & 7,500 & 276 & 0.44 & 0.02 & -0.48 & 6.45 & 0.00 & 4.07 & 0.87 & 64.80 & 23.91 & 0.00 & 0.00 & 0.00 & 0.00 & 0.00 & 0.00 & 98.1 \\
\hline & 11,000 & 301 & 0.91 & 0.55 & -0.47 & 16.37 & 0.00 & 10.34 & 0.70 & 56.62 & 15.96 & 0.00 & 0.00 & 0.00 & 0.00 & 0.01 & 0.01 & 94.4 \\
\hline & 11,000 & 326 & 1.46 & 2.07 & 0.43 & 22.09 & 0.00 & 13.70 & 1.15 & 63.06 & 0.00 & 0.01 & 0.00 & 0.01 & 0.00 & 0.02 & 0.03 & 99.9 \\
\hline \multirow{4}{*}{$\mathrm{RhMnTe} / \mathrm{SiO}_{2}$} & 7,500 & 256 & 0.00 & -0.26 & -0.26 & NA & NA & $\mathrm{NA}$ & NA & $\mathrm{NA}$ & NA & 0.00 & 0.00 & 0.00 & 0.00 & 0.00 & 0.00 & 104.1 \\
\hline & 7,500 & 277 & 0.00 & 0.49 & 0.49 & NA & NA & NA & NA & $\mathrm{NA}$ & NA & 0.00 & 0.00 & 0.00 & 0.00 & 0.00 & 0.00 & 106.3 \\
\hline & 11,000 & 300 & 0.15 & 1.07 & 0.91 & NA & NA & NA & NA & NA & NA & 0.00 & 0.00 & 0.00 & 0.00 & 0.00 & 0.00 & 106.9 \\
\hline & 11,000 & 326 & 0.00 & -0.25 & -0.25 & NA & NA & NA & NA & $\mathrm{NA}$ & NA & 0.00 & 0.00 & 0.00 & 0.00 & 0.00 & 0.00 & 98.3 \\
\hline \multirow{5}{*}{$\mathrm{RhMnBi} / \mathrm{SiO}_{2}$} & 7,500 & 257 & 0.00 & -0.06 & -0.06 & NA & NA & NA & NA & $\mathrm{NA}$ & NA & 0.00 & 0.00 & 0.00 & 0.00 & 0.00 & 0.00 & 100.1 \\
\hline & 7,500 & 276 & 0.00 & -0.74 & -0.74 & NA & NA & NA & NA & NA & NA & 0.00 & 0.00 & 0.00 & 0.00 & 0.00 & 0.00 & 96.4 \\
\hline & 11,000 & 301 & 0.00 & -0.45 & -0.45 & NA & NA & NA & NA & NA & NA & 0.00 & 0.00 & 0.00 & 0.00 & 0.00 & 0.00 & 98.9 \\
\hline & 11,000 & 325 & 0.54 & 0.48 & -0.14 & 4.76 & 0.00 & 5.43 & 4.03 & 76.17 & 10.09 & 0.00 & 0.00 & 0.00 & 0.00 & 0.00 & 0.00 & 99.1 \\
\hline & 11,000 & 345 & 0.88 & 1.01 & -0.01 & 9.69 & 0.04 & 11.29 & 8.96 & 62.14 & 8.94 & 0.00 & 0.00 & 0.01 & 0.00 & 0.00 & 0.01 & 99.1 \\
\hline \multirow{4}{*}{$\mathrm{RhMnPb} / \mathrm{SiO}_{2}$} & 7,500 & 257 & 0 & 0 & 0.92 & NA & NA & $\mathrm{NA}$ & NA & $\mathrm{NA}$ & $\mathrm{NA}$ & 0.00 & 0.00 & 0.00 & 0.00 & 0.00 & 0.00 & 106.1 \\
\hline & 7,500 & 276 & 0 & 0 & -0.36 & NA & NA & NA & NA & $\mathrm{NA}$ & $\mathrm{NA}$ & 0.00 & 0.00 & 0.00 & 0.00 & 0.00 & 0.00 & $98.3 !$ \\
\hline & 11,000 & 300 & 1.39 & 2.16 & 0.59 & 2.79 & 0.02 & 3.57 & 2.48 & 22.28 & 69.01 & 0.00 & 0.00 & 0.00 & 0.00 & 0.00 & 0.01 & 103.4 \\
\hline & 11,000 & 325 & 1.23 & 1.92 & 0.52 & 7.91 & 0.68 & 12.36 & 6.08 & 57.95 & 15.02 & 0.01 & 0.00 & 0.01 & 0.00 & 0.01 & 0.01 & 103.5 \\
\hline
\end{tabular}


Table A.1. (contd)

\begin{tabular}{|c|c|c|c|c|c|c|c|c|c|c|c|c|c|c|c|c|c|c|}
\hline \multirow[b]{2}{*}{ Catalyst } & \multirow[b]{2}{*}{$\begin{array}{c}\text { Space } \\
\text { Velocity } \\
\left(\mathrm{L} / \mathrm{L}_{\text {cat }} \mathrm{hr}\right)\end{array}$} & \multirow[b]{2}{*}{$\begin{array}{l}\text { Temp. } \\
\left({ }^{\circ} \mathrm{C}\right)\end{array}$} & \multirow[b]{2}{*}{$\begin{array}{l}\text { Carbon } \\
\text { Conv. } \\
(\%)\end{array}$} & \multirow[b]{2}{*}{$\begin{array}{c}\text { CO } \\
\text { Conv. } \\
(\%)\end{array}$} & \multirow{2}{*}{$\begin{array}{c}\mathrm{CO} \\
\text { Conv. } \\
\text { to } \mathrm{CO}_{2} \\
(\%)\end{array}$} & \multicolumn{6}{|c|}{ Carbon Selectivity (C-Mol\%) } & \multicolumn{6}{|c|}{$\mathrm{STY}\left(\mathrm{g} / \mathrm{mL}_{\mathrm{cat}} / \mathrm{hr}\right)$} & \multirow{2}{*}{$\begin{array}{c}\text { Carbon } \\
\text { Balance } \\
\left(\mathrm{C}_{\text {out }} / \mathrm{C}_{\text {in }}\right) \\
\%\end{array}$} \\
\hline & & & & & & $\mathrm{MeOH}$ & $\begin{array}{c}\text { Other } \mathrm{C}_{1} \\
\text { Oxygenates } \\
\end{array}$ & $\begin{array}{l}\mathrm{C}_{2}+ \\
\text { Alc. }\end{array}$ & $\begin{array}{c}\text { Other } \mathrm{C}_{2}+ \\
\text { Oxygenates } \\
\end{array}$ & $\mathrm{CH}_{4}$ & $\begin{array}{l}\text { Other } \\
\text { HCs } \\
\text { (est) }\end{array}$ & $\begin{array}{l}\mathrm{C}_{2}+ \\
\text { Alc. }\end{array}$ & $\begin{array}{c}\text { Other } \mathrm{C}_{2}+ \\
\text { Oxygenates } \\
\end{array}$ & $\begin{array}{c}\text { Total } \mathrm{C}_{2}+ \\
\text { Oxygenates } \\
\end{array}$ & $\begin{array}{c}\mathrm{HC} \\
\text { Liquids } \\
\end{array}$ & $\mathrm{MeOH}$ & $\begin{array}{l}\text { Total } \\
\text { Liq. } \\
\end{array}$ & \\
\hline \multirow{8}{*}{$\mathrm{RhMnAu} / \mathrm{SiO}_{2}$} & 7,500 & 257 & 6.86 & 7.01 & -0.77 & 0.59 & 0.00 & 7.52 & 47.48 & 35.21 & 9.20 & 0.01 & 0.10 & 0.11 & 0.00 & 0.00 & 0.11 & 94.3 \\
\hline & 7,500 & 276 & 18.36 & 20.80 & 0.09 & 0.26 & 0.00 & 5.57 & 30.35 & 34.12 & 29.70 & 0.03 & 0.16 & 0.19 & 0.04 & 0.00 & 0.22 & 96.1 \\
\hline & 11,000 & 300 & 27.28 & 30.46 & -0.48 & 0.35 & 0.00 & 8.09 & 34.16 & 38.21 & 19.19 & 0.09 & 0.40 & 0.49 & 0.03 & 0.01 & 0.52 & 98.2 \\
\hline & 11,000 & 316 & 32.22 & 35.89 & -0.60 & 0.24 & 0.00 & 8.02 & 23.13 & 50.83 & 17.78 & 0.10 & 0.31 & 0.41 & 0.02 & 0.00 & 0.44 & 94.1 \\
\hline & 15,000 & 314 & 24.84 & 27.94 & -0.15 & 0.45 & 0.00 & 8.62 & 27.89 & 46.96 & 16.07 & 0.11 & 0.39 & 0.50 & 0.01 & 0.01 & 0.52 & 98.0 \\
\hline & 7,500 & 276 & 10.70 & 11.72 & -0.42 & 6.01 & 0.00 & 13.64 & 24.75 & 45.16 & 10.44 & 0.04 & 0.07 & 0.11 & 0.00 & 0.02 & 0.14 & 96.9 \\
\hline & 15,000 & 327 & 24.09 & 27.09 & -0.09 & 0.62 & 0.00 & 9.64 & 18.44 & 58.47 & 12.84 & 0.12 & 0.25 & 0.37 & 0.00 & 0.01 & 0.38 & 93.5 \\
\hline & 19,000 & 325 & 19.33 & 21.80 & -0.08 & 0.81 & 0.00 & 10.20 & 20.90 & 56.06 & 12.03 & 0.13 & 0.28 & 0.42 & 0.00 & 0.01 & 0.43 & 97.2 \\
\hline \multirow{6}{*}{$\mathrm{RhMnV} / \mathrm{SiO}_{2}$} & 7,500 & 255 & 13.59 & 15.37 & 0.01 & 0.87 & 0.00 & 10.03 & 29.22 & 29.56 & 30.32 & 0.04 & 0.11 & 0.15 & 0.01 & 0.00 & 0.16 & 100.1 \\
\hline & 7,500 & 275 & 24.08 & 26.76 & -0.44 & 1.16 & 0.00 & 12.63 & 21.52 & 36.92 & 27.77 & 0.08 & 0.14 & 0.22 & 0.05 & 0.01 & 0.29 & 92.5 \\
\hline & 11,000 & 299 & 31.46 & 37.06 & 1.35 & 1.42 & 0.00 & 11.77 & 16.73 & 47.47 & 22.61 & 0.15 & 0.22 & 0.37 & 0.02 & 0.03 & 0.42 & 102.4 \\
\hline & 11,000 & 314 & 27.96 & 32.22 & 0.45 & 3.44 & 0.00 & 12.41 & 10.77 & 58.05 & 15.33 & 0.14 & 0.12 & 0.26 & 0.00 & 0.05 & 0.32 & 94.0 \\
\hline & 15,000 & 315 & 21.64 & 25.04 & 0.62 & 5.80 & 0.00 & 11.49 & 12.18 & 56.41 & 14.12 & 0.13 & 0.14 & 0.28 & 0.00 & 0.09 & 0.37 & 97.9 \\
\hline & 7,500 & 275 & 14.19 & 16.63 & 0.52 & 0.56 & 0.00 & 8.21 & 36.28 & 39.62 & 15.33 & 0.03 & 0.15 & 0.18 & 0.00 & 0.00 & 0.19 & 100.0 \\
\hline \multirow{4}{*}{$\mathrm{RhMnCs} / \mathrm{SiO}_{2}$} & 7,500 & 257 & 1.58 & 1.96 & 0.16 & 0.87 & 0.03 & 8.33 & 25.53 & 32.92 & 33.20 & 0.00 & 0.01 & 0.02 & 0.00 & 0.00 & 0.02 & 99.1 \\
\hline & 7,500 & 276 & 3.00 & 3.91 & 0.48 & 0.96 & 0.00 & 3.90 & 36.15 & 42.12 & 18.12 & 0.00 & 0.03 & 0.04 & 0.00 & 0.00 & 0.04 & 99.3 \\
\hline & 11,000 & 301 & 5.50 & 6.44 & 0.17 & 0.88 & 0.00 & 4.36 & 33.35 & 43.57 & 18.51 & 0.01 & 0.09 & 0.09 & 0.00 & 0.00 & 0.10 & 99.0 \\
\hline & 11,000 & 325 & 10.86 & 12.44 & 0.15 & 0.66 & 0.00 & 2.83 & 21.18 & 53.24 & 22.08 & 0.01 & 0.10 & 0.12 & 0.00 & 0.00 & 0.12 & 98.7 \\
\hline \multirow{4}{*}{$\mathrm{RhMnAg} / \mathrm{SiO}_{2}$} & 7,500 & 256 & 1.96 & 2.37 & 0.15 & 11.33 & 0.00 & 24.99 & 26.38 & 37.30 & 0.00 & 0.01 & 0.01 & 0.03 & 0.00 & 0.01 & 0.04 & 100.0 \\
\hline & 7,500 & 276 & 3.56 & 4.50 & 0.50 & 9.89 & 0.00 & 18.18 & 16.88 & 55.05 & 0.00 & 0.02 & 0.02 & 0.03 & 0.00 & 0.01 & 0.05 & 98.2 \\
\hline & 11,000 & 300 & 5.78 & 7.55 & 1.03 & 8.08 & 0.00 & 16.30 & 12.80 & 62.82 & 0.00 & 0.04 & 0.03 & 0.07 & 0.00 & 0.03 & 0.09 & 104.8 \\
\hline & 11,000 & 325 & 12.22 & 13.68 & -0.18 & 4.31 & 0.00 & 13.20 & 8.13 & 64.13 & 10.23 & 0.06 & 0.04 & 0.11 & 0.00 & 0.03 & 0.14 & 101.5 \\
\hline
\end{tabular}

(a) Sample tested in previous screening tests (Gerber et al. 2007)

(b) Test conducted using clamshell furnace rather than hot oil circulation.

(c) Silica was obtained from Altech, Inc. 

Appendix B

\section{Summary of Individual Test Results}





\section{Appendix B}

\section{Summary of Individual Test Results}

A total of 28 tests examining 22 different catalyst promoters as well as the unpromoted catalyst were evaluated during this phase of testing. This appendix discusses conditions under which each test was conducted and the performance of the catalyst under these conditions. A summary of the conditions evaluated in each test along with the reported carbon conversions STYs and carbon selectivities are summarized in Appendix A.

\section{B.1 Unmodified RhMn/SiO 2 Catalyst}

Two tests were conducted using the unmodified $\mathrm{Rh}-\mathrm{Mn} / \mathrm{SiO}_{2}$ catalyst; both of these tests used circulated hot oil to heat the reactor. The first test was conducted to establish a performance baseline for the unmodified catalyst under conditions comparable to those of the promoted catalysts prepared using the Altech Inc. source of Davisil $625 \mathrm{SiO}_{2}$. The catalyst testing sequence was 256 and $277^{\circ} \mathrm{C}$ at 7500 at $\mathrm{L} / \mathrm{L}_{\text {cat }} / \mathrm{hr} ; 300,317,314$, and $324^{\circ} \mathrm{C}$ at $11,000 \mathrm{~L} / \mathrm{L}_{\mathrm{cat}} / \mathrm{hr} ; 324,315$, and $302^{\circ} \mathrm{C}$ at $15,000 \mathrm{~L} / \mathrm{L}_{\mathrm{cat}} / \mathrm{hr} ; 302^{\circ} \mathrm{C}$ at $11,000 \mathrm{~L} / \mathrm{L}_{\text {cat }} / \mathrm{hr}$; and $256^{\circ} \mathrm{C}$ at $7500 \mathrm{~L} / \mathrm{L}_{\text {cat }} / \mathrm{hr}$. The second test was a repeat of the first test to investigate whether the second batch of Davisil $625 \mathrm{SiO}_{2}$ obtained from Fisher Scientific affected the performance of the unmodified catalyst. The catalyst testing sequence for the second catalyst was 255 and $276^{\circ} \mathrm{C}$ at 7500 at $\mathrm{L} / \mathrm{L}_{\text {cat }} / \mathrm{hr} ; 299,314$, and $315^{\circ} \mathrm{C}$ at $11,000 \mathrm{~L} / \mathrm{L}_{\text {cat }} / \mathrm{hr}$; and $275^{\circ} \mathrm{C}$ at $7500 \mathrm{~L} / \mathrm{L}_{\text {cat }} / \mathrm{hr}$.

Figure B.1 shows the carbon conversion for both tests using the unmodified $\mathrm{Rh}-\mathrm{Mn} / \mathrm{SiO}_{2}$ catalysts. It can be seen that carbon conversion for both $\mathrm{Rh}-\mathrm{Mn} / \mathrm{SiO}_{2}$ catalyst achieves a regular increase in carbon conversion with temperature and a lower conversion at higher space velocities, as would be expected. The repeat conditions for both catalysts also suggest that there was some deactivation of the catalyst with respect to carbon conversion over the durations of the tests.

Figure B. 2 shows the $\mathrm{C}_{2}+$-oxygenate $\mathrm{STYs}$ for both catalysts. It appears that while the STYs increased with increasing temperature up to about $300^{\circ} \mathrm{C}$ for both catalysts, higher temperatures produced no improvement for the first catalyst tested and some decrease in performance for the second catalyst tested (i.e., from the second $\mathrm{SiO}_{2}$ batch). Increasing the space velocity to reduce carbon conversion did not improve the STY in the first test, suggesting an overall deactivation of the catalyst at the higher temperatures. Repeat of test conditions at lower temperatures for both catalysts confirm this hypothesis. 


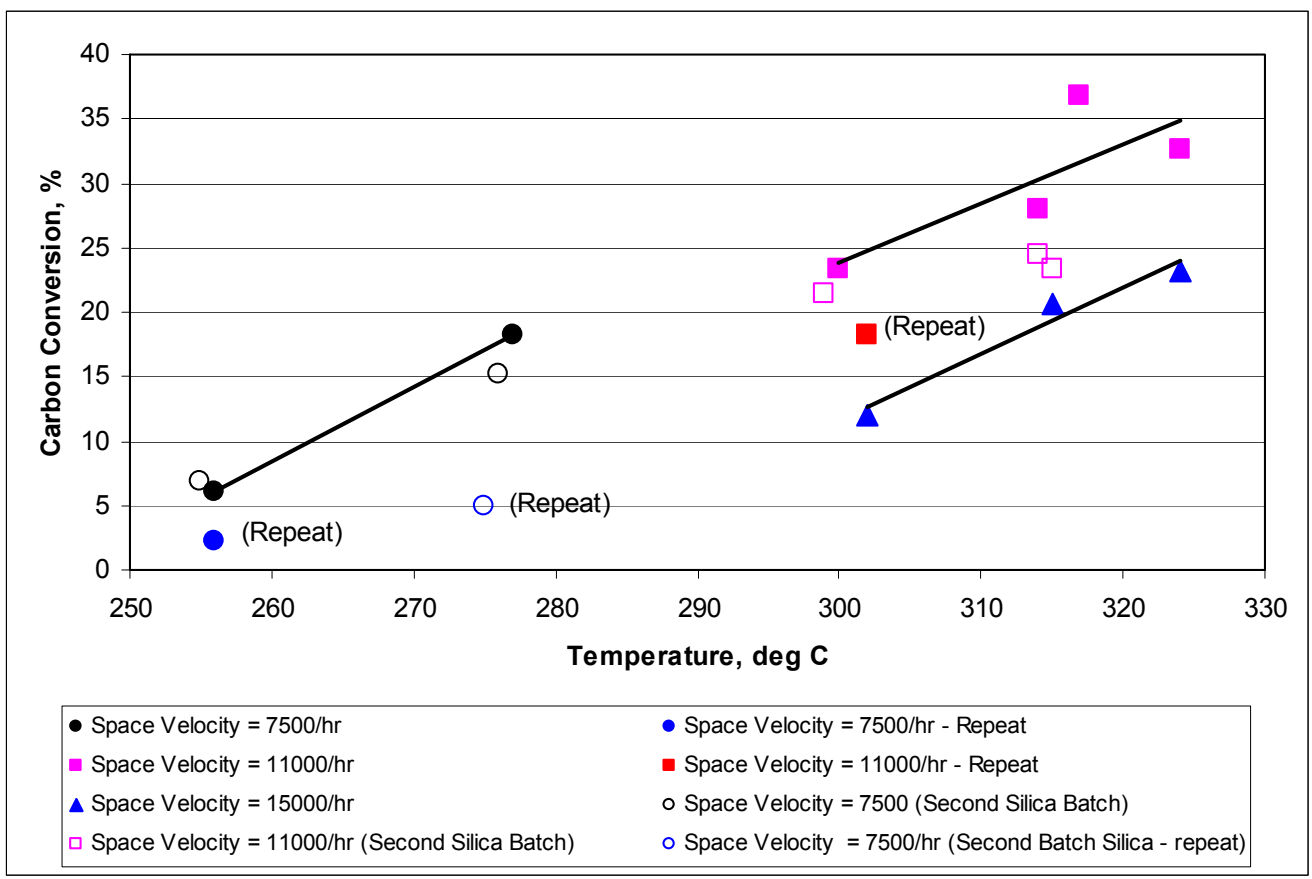

Figure B.1. Carbon Conversions for the $\mathrm{Rh} / \mathrm{Mn} / \mathrm{SiO}_{2}$ Catalysts

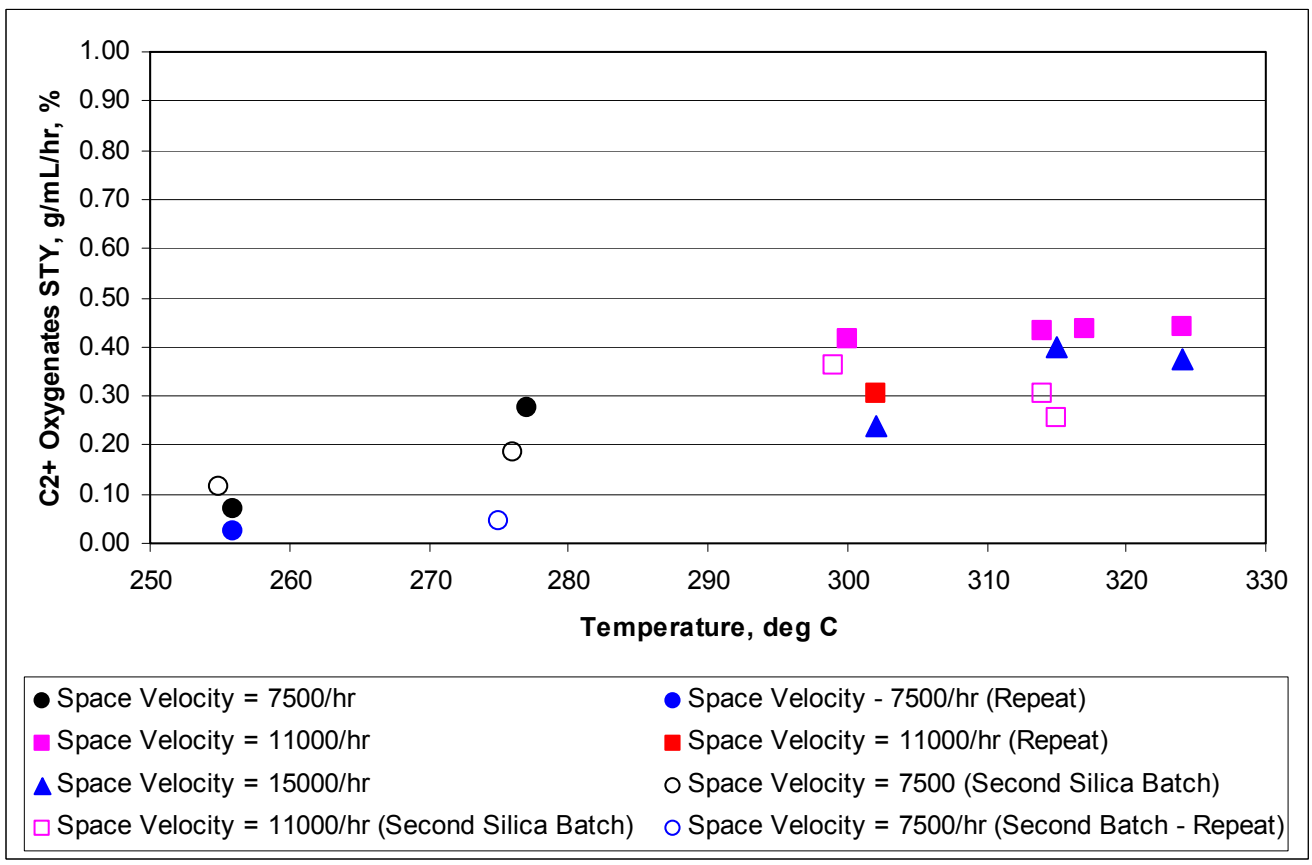

Figure B.2. $\mathrm{C}_{2}+-$ Oxygenate $\mathrm{STYs}$ for $\mathrm{Rh} / \mathrm{Mn} / \mathrm{SiO}_{2}$ Catalysts

Figure B.3 shows the carbon selectivity to $\mathrm{C}_{2}+$ oxygenates. Also shown in the figure is the best fit trend line for all of the data for all test temperatures except approximately $255^{\circ} \mathrm{C}$ for the first catalyst tested. It appears that both catalysts show a similar trend of decreasing selectivity to $\mathrm{C}_{2}+$ oxygenates with increasing temperature with the exception the first catalyst operating at approximately $255^{\circ} \mathrm{C}$, including the repeat condition, and the second catalyst repeat condition at approximately $275^{\circ} \mathrm{C}$. The second catalyst tested also appears to have a slightly lower selectivity to $\mathrm{C}_{2}+$ oxygenates. 


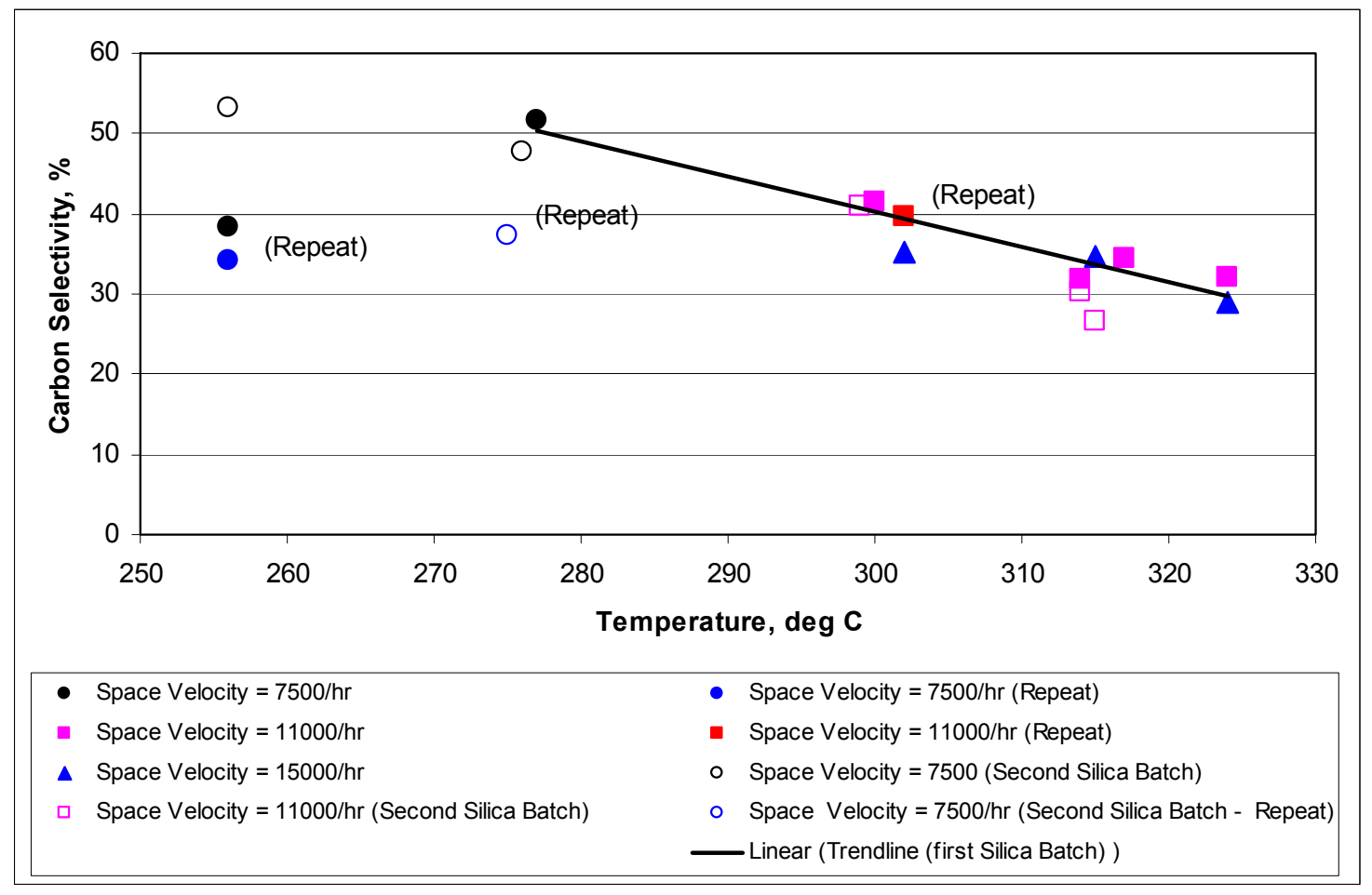

Figure B.3. Carbon Selectivity to $\mathrm{C}_{2}+$ Oxygenates for $\mathrm{Rh} / \mathrm{Mn} / \mathrm{SiO}_{2}$ Catalysts

Figure B.4 shows the carbon selectivity of all oxygenates to $\mathrm{C}_{2}+$ alcohols. Also shown in the figure is the best fit trend line for each catalyst for all test temperatures except approximately $255^{\circ} \mathrm{C}$ for the first catalyst tested. It appears both catalysts show similar trends of increasing selectivity to $\mathrm{C}_{2}+$ alcohols with increasing temperature with the exception the first catalyst operating at approximately $255^{\circ} \mathrm{C}$, including the repeat condition. However, there is a pronounced difference in the selectivity of oxygenates to $\mathrm{C}_{2}+$ alcohols with the second catalyst having a significantly higher selectivity to $\mathrm{C}_{2}+$ alcohols at comparable reaction conditions for both catalysts. The second catalyst also shows a significant increase in the methanol yields when compared to the first catalyst as shown in Figure B.5. Similarly, the carbon selectivity to hydrocarbon liquids is eliminated in the second catalyst at all temperatures, and the carbon selectivity to higher hydrocarbon gases (ethane, propane, etc.) also is significantly reduced. Figure B.5 also shows that the production of liquid hydrocarbons in the first catalyst are much lower at temperatures above $300^{\circ} \mathrm{C}$, suggesting a deactivation of the catalyst with respect to hydrocarbon chain growth. At this time, there is no good explanation of the carbon selectivity behavior or the first catalyst at approximately $255^{\circ} \mathrm{C}$. The quantity of liquid collected at this temperature for both the first sample and the sample for the repeat condition was significantly less than the quantity of liquid collected for the other test conditions of both samples $\left(1.6\right.$ and $0.6 \mathrm{~g}$ of condensate at $255^{\circ} \mathrm{C}$ for the first catalyst vs. $2.9 \mathrm{~g}$ at approximately $255^{\circ} \mathrm{C}$ for the second catalyst). This could have produced a greater fraction of unrecovered condensate because of the wetted walls of the trap, resulting in a lower apparent yield of $\mathrm{C}_{2}+$ oxygenates at these conditions. For now, the behavior is being treated as an anomaly in the overall trend of carbon selectivity to $\mathrm{C}_{2}+$ oxygenates with temperature. 


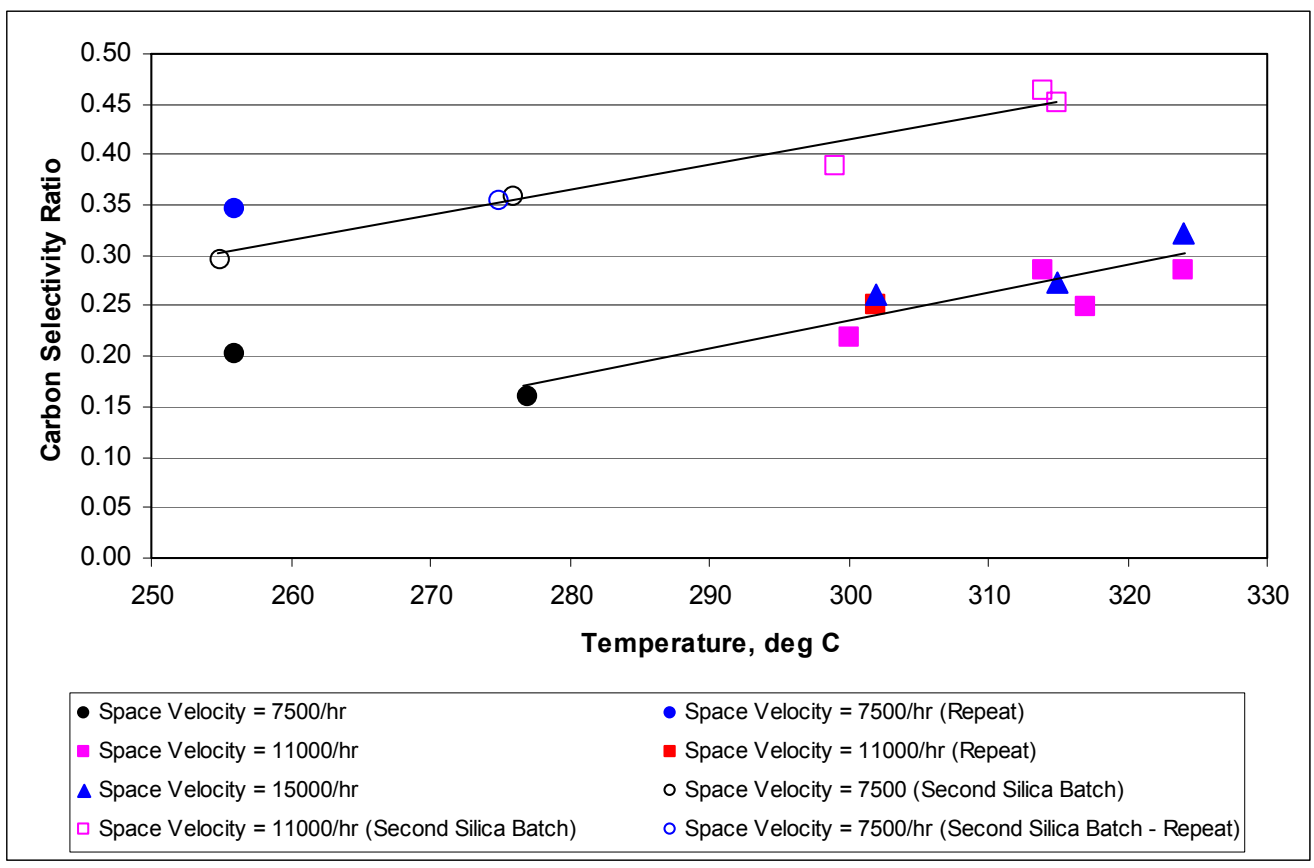

Figure B.4. Selectivity of the All Oxygenates to $\mathrm{C}_{2}+$ Alcohols

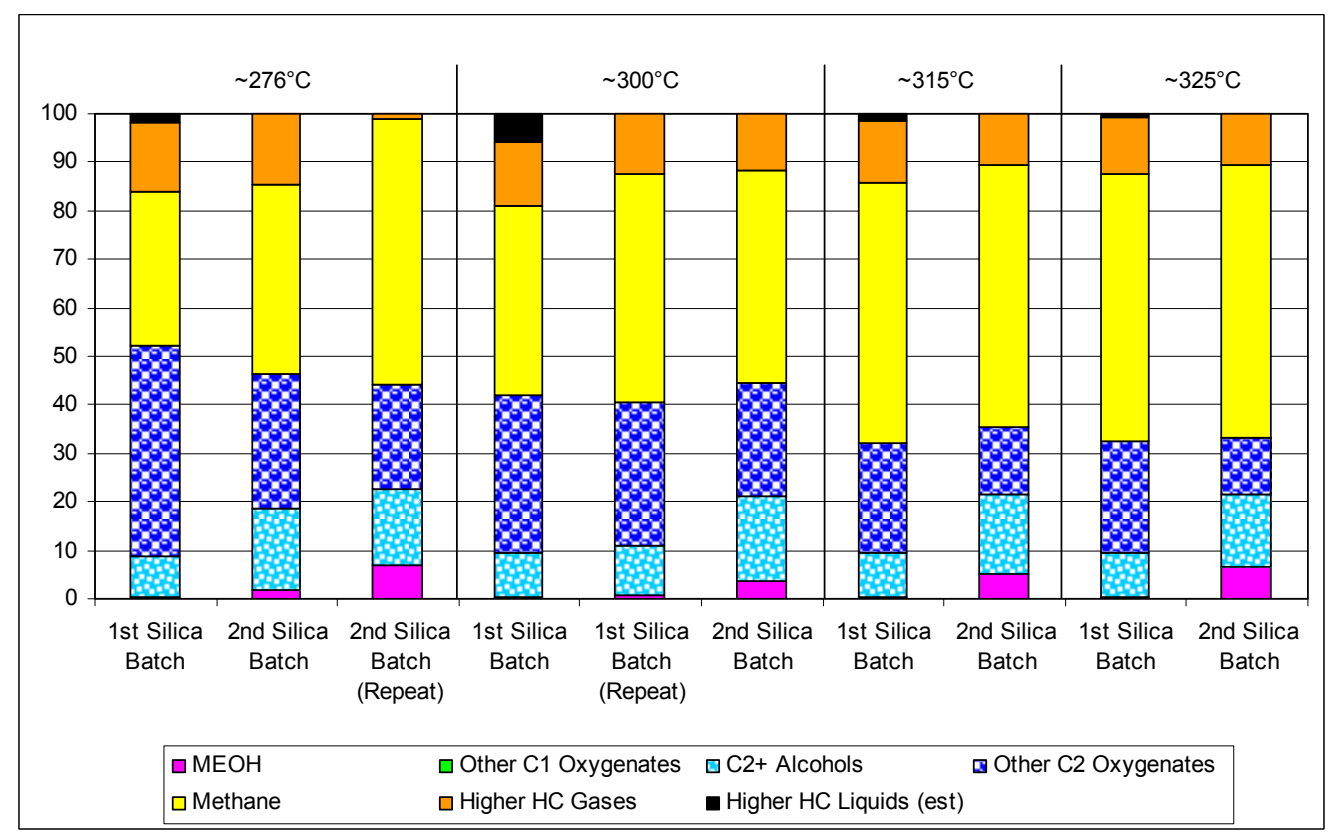

Figure B.5. Carbon Selectivity to Liquid Products for the Rh-Mn/SiO 2 Catalysts

\section{B.2 Fe-Promoted Catalysts}

Two Fe-promoted catalysts were tested this year. The first catalyst $\left(\mathrm{RhMn} / \mathrm{SiO}_{2} \mathrm{~A}\right)$ was prepared in the same manner as the $\left(\mathrm{RhMnFe} / \mathrm{SiO}_{2}\right)$ catalyst that was tested during the initial catalyst screening except that a new batch of the base catalyst was used. The purpose of the testing was to verify that the 
two batches of Fe-promoted catalysts behaved similarly. Details of the performance of the $\mathrm{RhMnFe} / \mathrm{SiO}_{2}$ catalyst are discussed by Gerber et al. (2007). The second catalyst $\left(\mathrm{RhMn} / \mathrm{SiO}_{2} \mathrm{~B}\right)$ had the same composition as the other two catalysts except that the support was Davisil $\mathrm{LC} 150 \mathrm{SiO}_{2}$ instead of Davisil $645 \mathrm{SiO}_{2}$, and the Fe precursor was co-impregnated with the $\mathrm{Rh}$ and $\mathrm{Mn}$ precursors in a single impregnation. The previous two catalysts were co-impregnated with the Rh and Mn precursors and the catalyst dried before a adding the Fe in a second impregnation. All of the tests were conducted using the furnace to heat the catalysts. Also, gas mixtures evaluated ranged from 1.8 to 2.0 for those tested using a low $\mathrm{H}_{2}$ : $\mathrm{CO}$ ratio feed gas, and from 2.3 to 2.6 for those test using a high $\mathrm{H}_{2}: \mathrm{CO}$ ratio feed gas. The two different ranges of gas composition were used to compare data obtained in earlier screening tests. The testing sequence for the $\mathrm{RhMnFe} / \mathrm{SiO}_{2}$ catalyst was $257^{\circ} \mathrm{C}$ at $7400 \mathrm{~L} / \mathrm{L}_{\text {cat }} / \mathrm{hr}, 257$ at $15,000 \mathrm{~L} / \mathrm{L}_{\text {cat }} / \mathrm{hr}$, $285^{\circ} \mathrm{C}$ at $7,400 \mathrm{~L} / \mathrm{L}_{\text {cat }} / \mathrm{hr}$, and 323 and $326^{\circ} \mathrm{C}$ at $11,000 \mathrm{~L} / \mathrm{L}_{\text {cat }} / \mathrm{hr}$, all using a syngas with a $2.5: 2.6 \mathrm{H}_{2}: \mathrm{CO}$ ratio; $326^{\circ} \mathrm{C}$ at 11,000 and $15,000 \mathrm{~L} / \mathrm{L}_{\text {cat }} / \mathrm{hr}$ using a syngas with a $2.0 \mathrm{H}_{2}: \mathrm{CO}$ ratio; and $354^{\circ} \mathrm{C}$ and $402^{\circ} \mathrm{C}$ at $15,000 \mathrm{~L} / \mathrm{L}_{\text {cat }} / \mathrm{hr}$ using a syngas with a $2.4 \mathrm{H}_{2}$ : CO ratio. The testing sequence for the $\mathrm{RhMnFe} / \mathrm{SiO}_{2} \mathrm{~A}$ catalyst was 257 and $275^{\circ} \mathrm{C}$ at $7500 \mathrm{~L} / \mathrm{L}_{\text {cat }} / \mathrm{hr}, 325$ and $325^{\circ} \mathrm{C}$ at $11,000 \mathrm{~L} / \mathrm{L}_{\text {cat }} / \mathrm{hr}$, and $301^{\circ} \mathrm{C}$ at $9300 \mathrm{~L} / \mathrm{L}_{\text {cat }} / \mathrm{hr}$, all using a syngas with a $2.4 \mathrm{H}_{2}: \mathrm{CO}$ ratio. The testing sequence for the $\mathrm{RhMnFe} / \mathrm{SiO}_{2} \mathrm{~B}$ catalyst was 255 and $275^{\circ} \mathrm{C}$ at $7400 \mathrm{~L} / \mathrm{L}_{\text {cat }} / \mathrm{hr}$, and $327^{\circ} \mathrm{C}$ at $11,000 \mathrm{~L} / \mathrm{L}_{\text {cat }} / \mathrm{hr}$, all using a syngas with a $2.4 \mathrm{H}_{2}$ : $\mathrm{CO}$ ratio; 325 and $300^{\circ} \mathrm{C}$ at 11,000 using a syngas with a $1.9 \mathrm{H}_{2}: \mathrm{CO}$ ratio; and $300^{\circ} \mathrm{C}$ at $11,000 \mathrm{~L} / \mathrm{L}_{\text {cat }} / \mathrm{hr}$ and $275^{\circ} \mathrm{C}$ at $7500 \mathrm{~L} / \mathrm{L}_{\text {cat }} / \mathrm{hr}$ using a syngas with a $2.4 \mathrm{H}_{2}: \mathrm{CO}$ ratio.

Figure B.6 shows that carbon conversion increased with temperature and decreased with increasing space velocity, as expected. There was also a modest decrease in the carbon conversion with decreasing $\mathrm{H}_{2}$ :CO ratio.

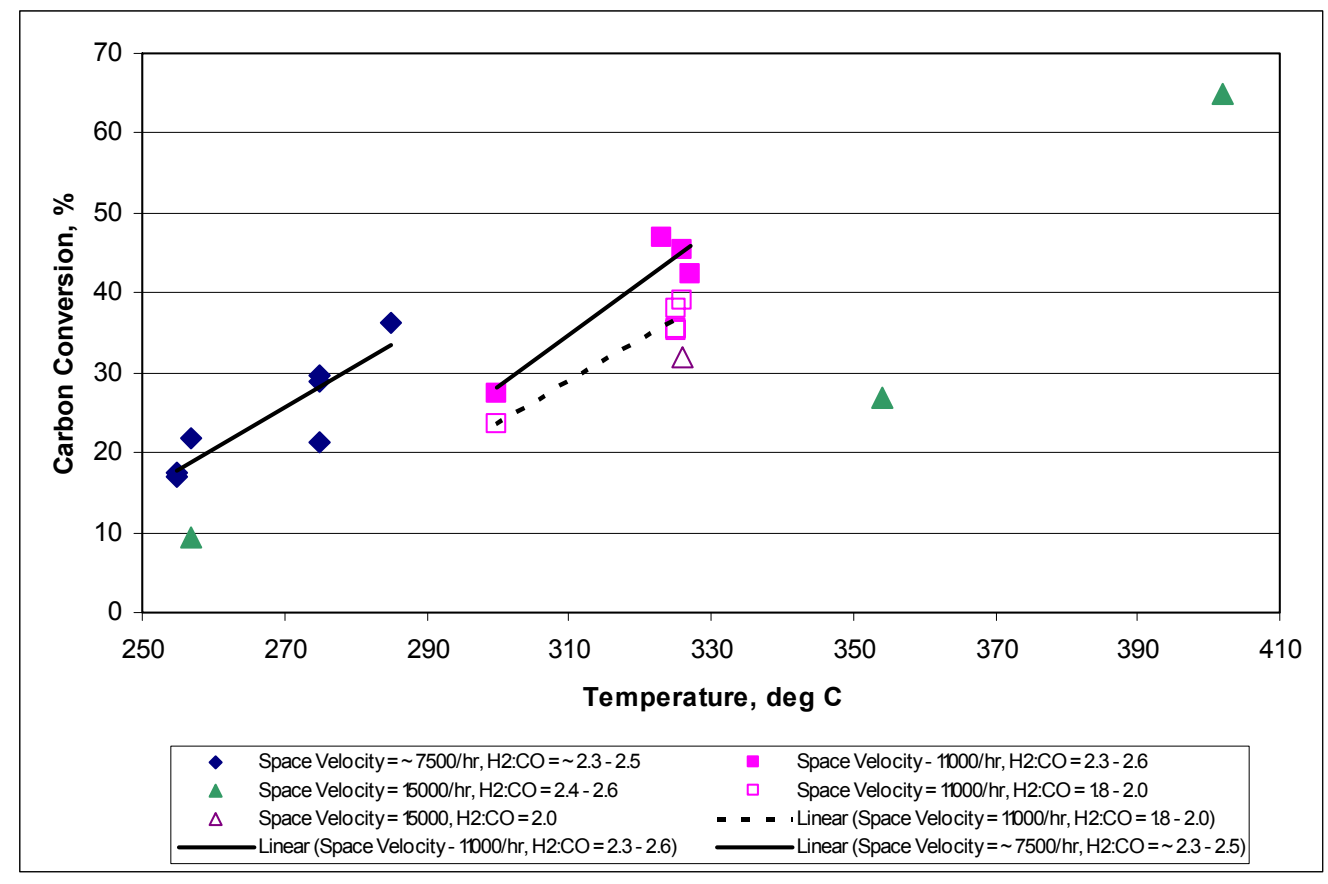

Figure B.6. Carbon Conversion for the Fe-Promoted Catalysts 
Figure B.7 and B.8 compare the $\mathrm{C}_{2}+$-oxygenate STYs and carbon selectivities, respectively, for the three $\mathrm{Fe}$-promoted catalysts. It appears that there are no significant differences in the $\mathrm{C}_{2}+$-oxygenate STYs or selectivities of the three catalysts with respect to the $\mathrm{SiO}_{2}$ support, and the method of preparation. There also does not appear to be a significant effect of the $\mathrm{H}_{2}$ :CO ratio on either the STYs or carbon selectivities.

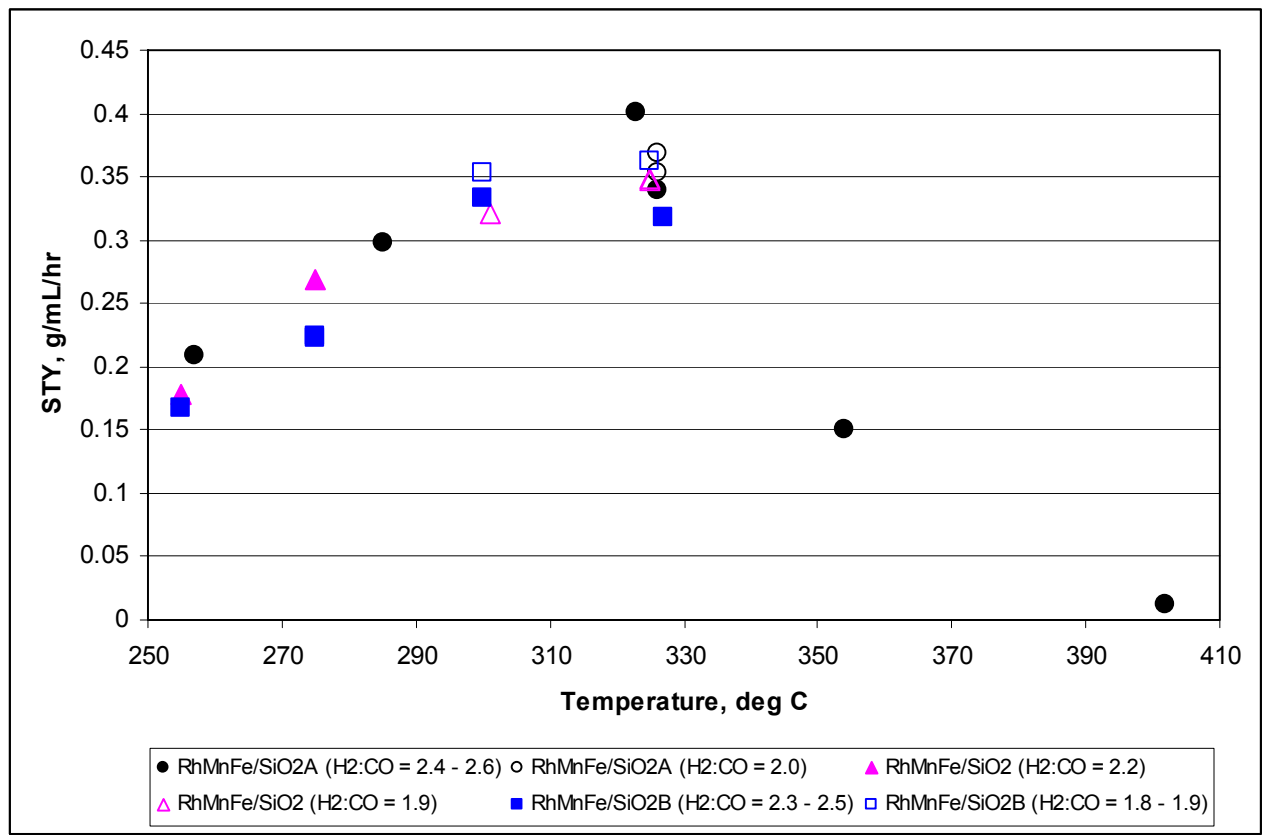

Figure B.7. $\mathrm{C}_{2}+-$-Oxygenate STYs for Fe-Promoted Catalysts

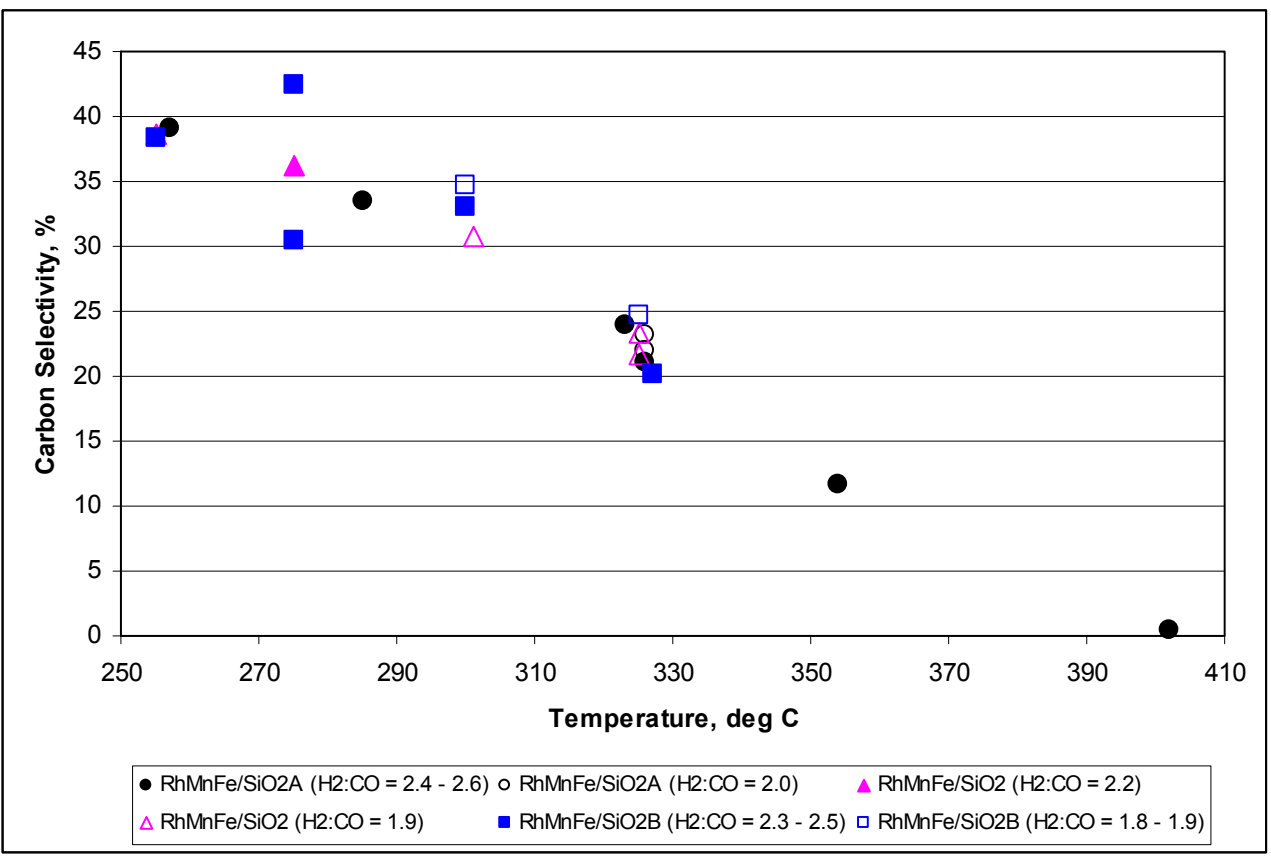

Figure B.8. Carbon Selectivities to $\mathrm{C}_{2}+$ Oxygenates for Fe-Promoted Catalysts 
Figure B.9 shows that increasing the catalyst temperature increases the carbon selectivity to $\mathrm{C}_{2}+$ alcohols relative to the other oxygenates, although the selectivity may remain constant above approximately $330^{\circ} \mathrm{C}$ catalyst temperature. The figure also suggests that the $\mathrm{H}_{2}: \mathrm{CO}$ ratio has a modest effect on this selectivity with higher $\mathrm{H}_{2}$ :CO ratios producing higher selectivities to $\mathrm{C}_{2}+$ alcohols (best illustrated by the trend lines in the figure for testing conditions at $11,000 \mathrm{~L} / \mathrm{L}_{\text {cat }} / \mathrm{hr}$ space velocity. There is no clear trend on the effect of the space velocity on the carbon selectivity to $\mathrm{C}_{2}+$ alcohols relative to the other oxygenates.

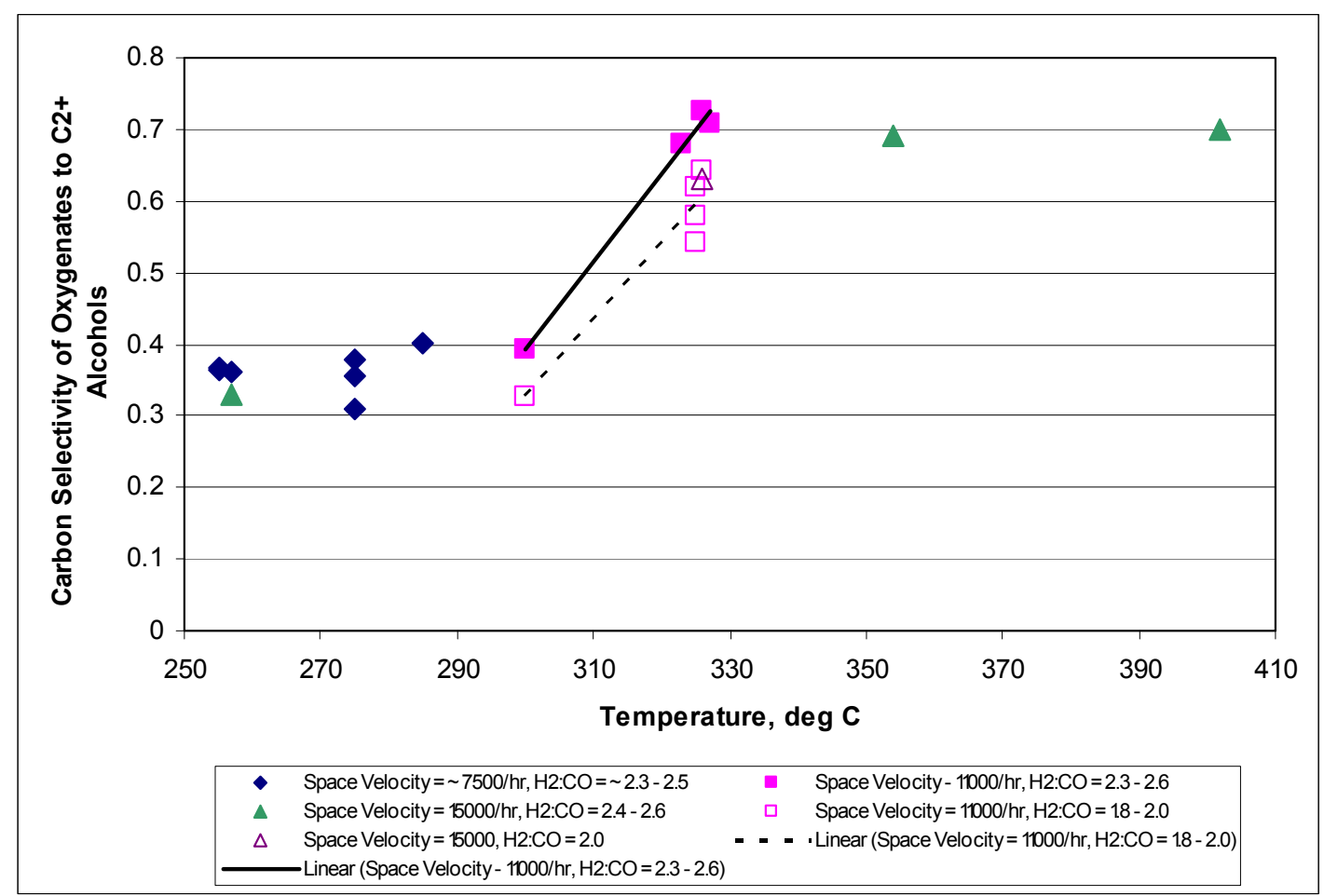

Figure B.9. Carbon Selectivity of All Oxygenates to $\mathrm{C}_{2}+$ Alcohols

Another trend noted for both the $\mathrm{RhMnFe} / \mathrm{SiO}_{2}$ and $\mathrm{RhMnFe} / \mathrm{SiO}_{2} \mathrm{~B}$ catalysts was a significant decrease in the carbon selectivity to liquid hydrocarbons when the catalyst temperature was elevated above approximately 275 to $300^{\circ} \mathrm{C}$ or higher (Figure B.9A). This decrease was not observed for the $\mathrm{RhMnFe} / \mathrm{SiO}_{2}$ catalyst tested during the previous screening tests, although it is possible that the organic liquids were not clearly observed and, thus, not separated from the liquid samples in that test. 


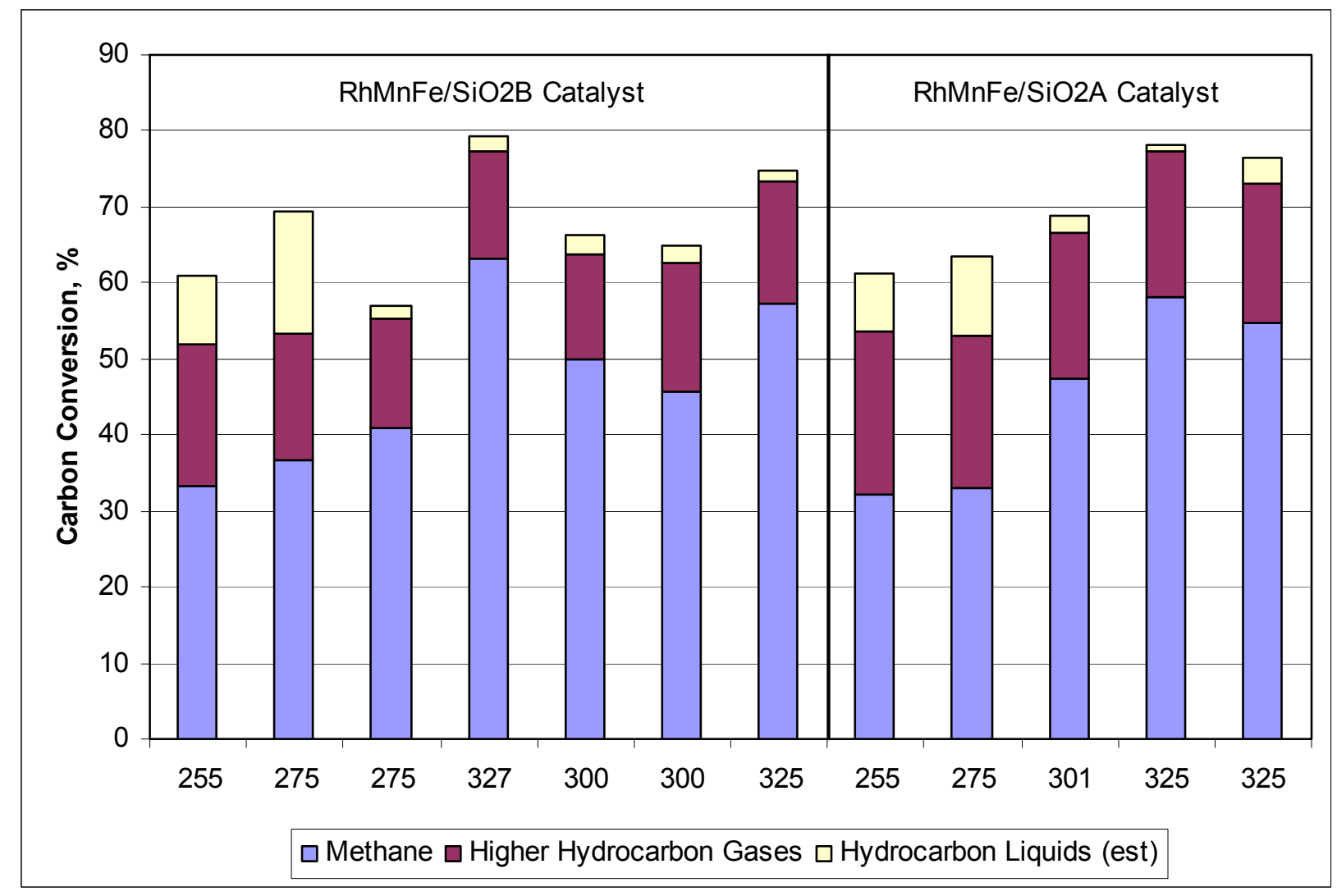

Figure B.9A. Comparison of Hydrocarbon Selectivities for Fe-Promoted Catalysts

\section{B.3 Li-Promoted Catalysts}

Two Li-promoted catalysts were tested. Both catalysts were prepared in the same manner except that one catalyst $\left(\mathrm{RhMnLi} / \mathrm{SiO}_{2}\right)$ was reduced at a maximum temperature of $260^{\circ} \mathrm{C}$ while the second catalyst $\left(\mathrm{RhMnL} 1 / \mathrm{SiO}_{2} \mathrm{~A}\right.$ ) was reduced at a maximum temperature of $350^{\circ} \mathrm{C}$ (see Section 2.2 for description of catalyst reduction). Both catalysts were tested using the furnace to heat the reactor.

During the first catalyst test, samples were collected for catalyst temperature conditions of 256 and $277^{\circ} \mathrm{C}$, at $7500 \mathrm{~L} / \mathrm{L}_{\text {cat }} / \mathrm{hr}$, and $302^{\circ} \mathrm{C}$ (two sets of samples) at $11,000 \mathrm{~L} / \mathrm{L}_{\text {cat }} / \mathrm{hr}$. When the furnace temperature was increased following collection of the fourth sample, a temperature excursion of 80 to $90^{\circ} \mathrm{C}$ occurred that remained above $375^{\circ} \mathrm{C}$ for three $\mathrm{hr}$ and then slowly cooled to $344^{\circ} \mathrm{C}$ over the next 18 hours. An attempt to elevate the temperature to $350^{\circ} \mathrm{C}$ (at $11,000 \mathrm{~L} / \mathrm{L}_{\text {cat }} / \mathrm{hr}$ ) was then attempted, and the temperature remained there for 7 hours before abruptly dropping to $297^{\circ} \mathrm{C}$. The catalyst temperature was stabilized at approximately $293^{\circ} \mathrm{C}$, long enough to collect a representative sample and associated data set. It was clear from the quantity of liquid in this sample that the catalyst had deactivated, so two additional test conditions were obtained at 326 and $350^{\circ} \mathrm{C}$ at $11,000 \mathrm{~L} / \mathrm{L}_{\text {cat }} / \mathrm{hr}$ to examine the performance of the deactivated catalyst. The second catalyst was tested similarly to the first catalyst prior to its temperature excursion to examine whether reducing the catalyst at a higher temperature reduced its activity. A $350^{\circ} \mathrm{C}$ maximum reduction temperature was selected because the $\mathrm{Fe}$-promoted catalysts experienced decreases in the liquid hydrocarbon yield at catalyst temperatures at or above $300^{\circ} \mathrm{C}$. 
The testing sequence for the second catalyst was 257 and $277^{\circ} \mathrm{C}$, at $7500 \mathrm{~L} / \mathrm{L}_{\text {cat }} / \mathrm{hr}$, and 300 and $305^{\circ} \mathrm{C}$ at $11,000 \mathrm{~L} / \mathrm{L}_{\text {cat }} / \mathrm{hr}$.

Figures B.10 and B.11 show the effects of temperature on the carbon conversions and STYs of both catalysts. It can be seen that both carbon conversion and $\mathrm{C}_{2}+$ oxygenates STYs decreased following the temperature excursion, which is indicative of catalyst deactivation. However, it was possible to bring carbon conversion to pre-temperature excursion levels at a higher temperature $\left(350^{\circ} \mathrm{C}\right.$ vs. approximately $300^{\circ} \mathrm{C}$ prior to the temperature excursion), although the $\mathrm{C}_{2}+$-oxygenate $\mathrm{STYs}$ were about $20 \%$ lower than before for the same carbon conversion. Reducing the catalyst at a higher temperature appeared to have a slightly negative effect on the carbon conversion and $\mathrm{C}_{2}+$-oxygenate STYs at temperatures up to approximately $300^{\circ} \mathrm{C}$.

Interestingly, the $\mathrm{C}_{2}+$-oxygenate carbon selectivity trend with temperature for the catalyst before and after the temperature excursion remained the same according to Figure B.11A. On the other hand, the $\mathrm{C}_{2}+$ alcohols accounted for a significantly greater portion of the total oxygenates after the temperature excursion as shown in Figure B.12. Reducing the catalyst at a higher temperature did not have a significant effect on the carbon selectivity to $\mathrm{C}_{2}+$ oxygenates and a slightly positive effect on the fraction of the oxygenates that were $\mathrm{C}_{2}+$ alcohols.

Another observation was the effect of temperature on the carbon selectivity to various hydrocarbons. Figure B.13 shows that the following the temperature excursion, the carbon selectivity to hydrocarbon liquids was eliminated and the selectivity to $\mathrm{C}_{2}-\mathrm{C}_{5}$ hydrocarbon gases was diminished even at the higher catalyst temperatures when the total carbon selectivity to hydrocarbons was as high as $60 \%$. When taken together with the carbon selectivity to $\mathrm{C}_{2}+$-oxygenate trend, which was not affected by the temperature excursion (Figure B.11A), it appears that the excursion altered the carbon chain-growth mechanism for hydrocarbons while not affecting the chain-growth mechanism for oxygenates. This suggests that different catalyst sites are responsible for $\mathrm{C}_{2}+$-hydrocarbon and $\mathrm{C}_{2}+$-oxygenate synthesis. Reducing the catalyst at a higher temperature appeared to result in a slight reduction in the carbon selectivity to higher hydrocarbons as shown in Figure B.13.

Overall, it appeared that increasing the reducing temperature of the catalyst resulted in relatively minor negative effects on the catalyst performance in terms of carbon conversion and $\mathrm{C}_{2}+$-oxygenate STYs, no effect on carbon selectivity to $\mathrm{C}_{2}+$ oxygenates, and significant positive effects in terms of higher ratio of $\mathrm{C}_{2}+$ alcohols to total oxygenates and reduced production of higher hydrocarbons. Based on these results, subsequent testing used the higher reduction temperature procedure. 


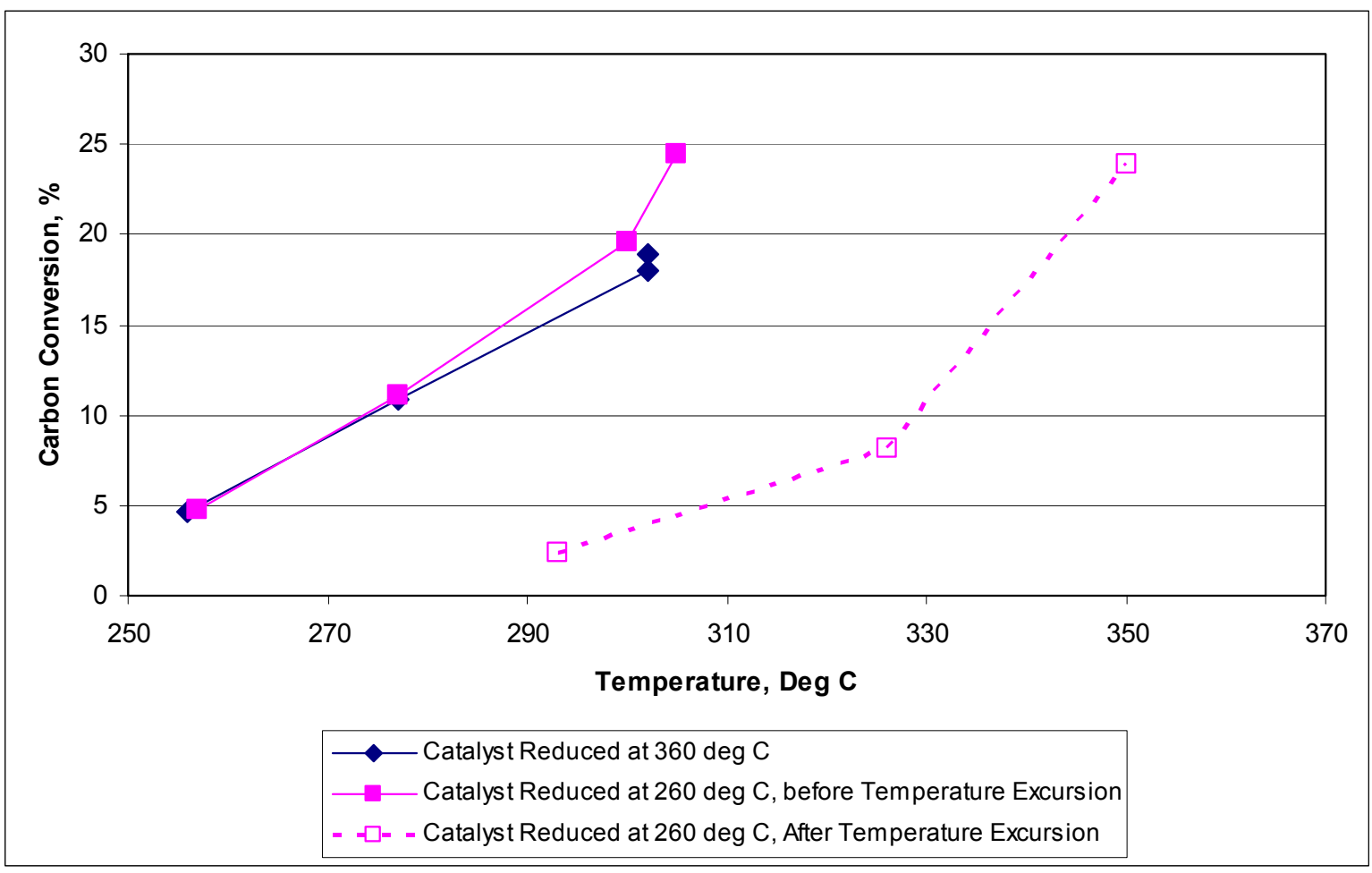

Figure B.10. Carbon Conversion of Li-Promoted Catalysts

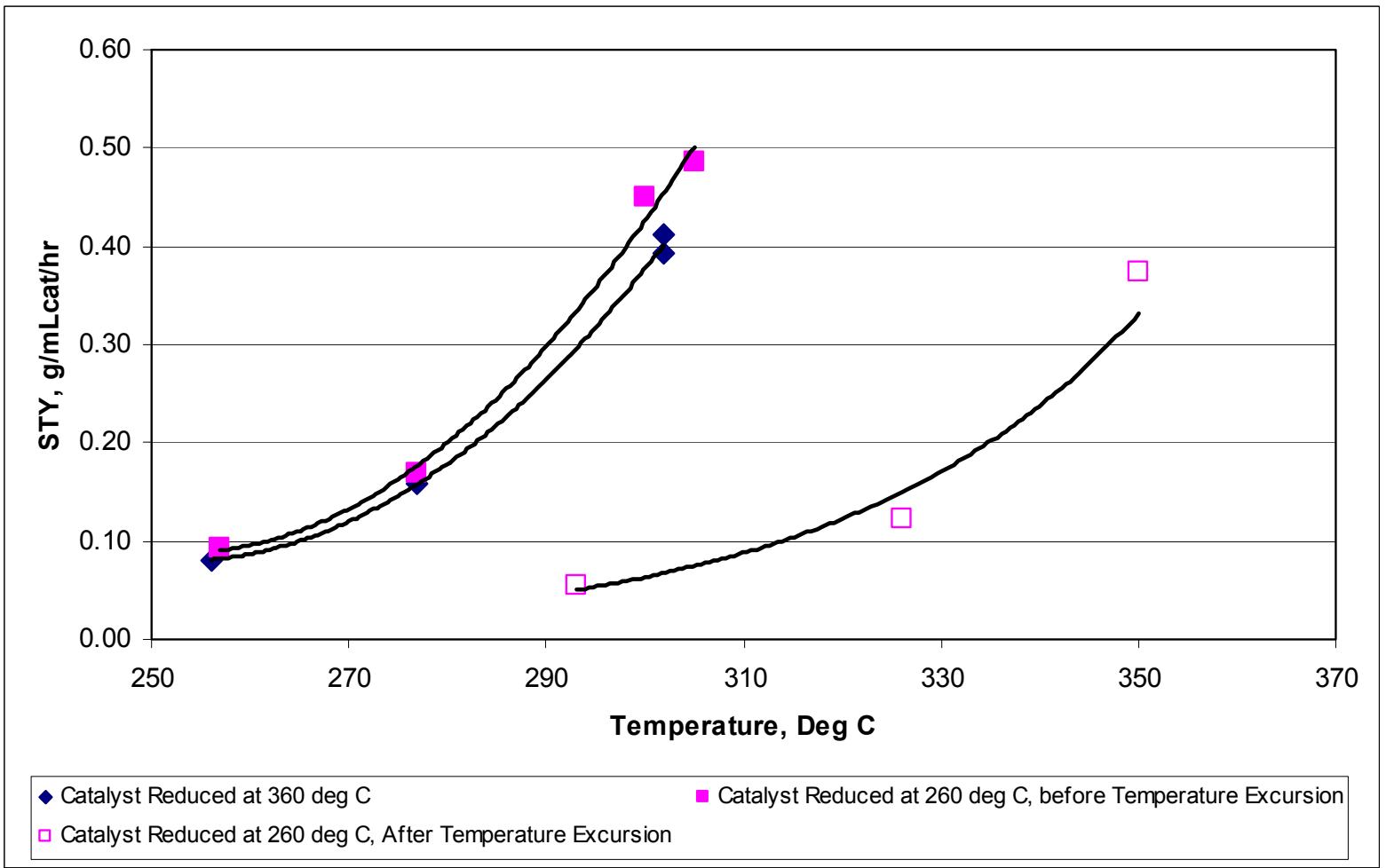

Figure B.11. $\mathrm{C}_{2}+-$ Oxygenate STYs for Li-Promoted Catalysts 


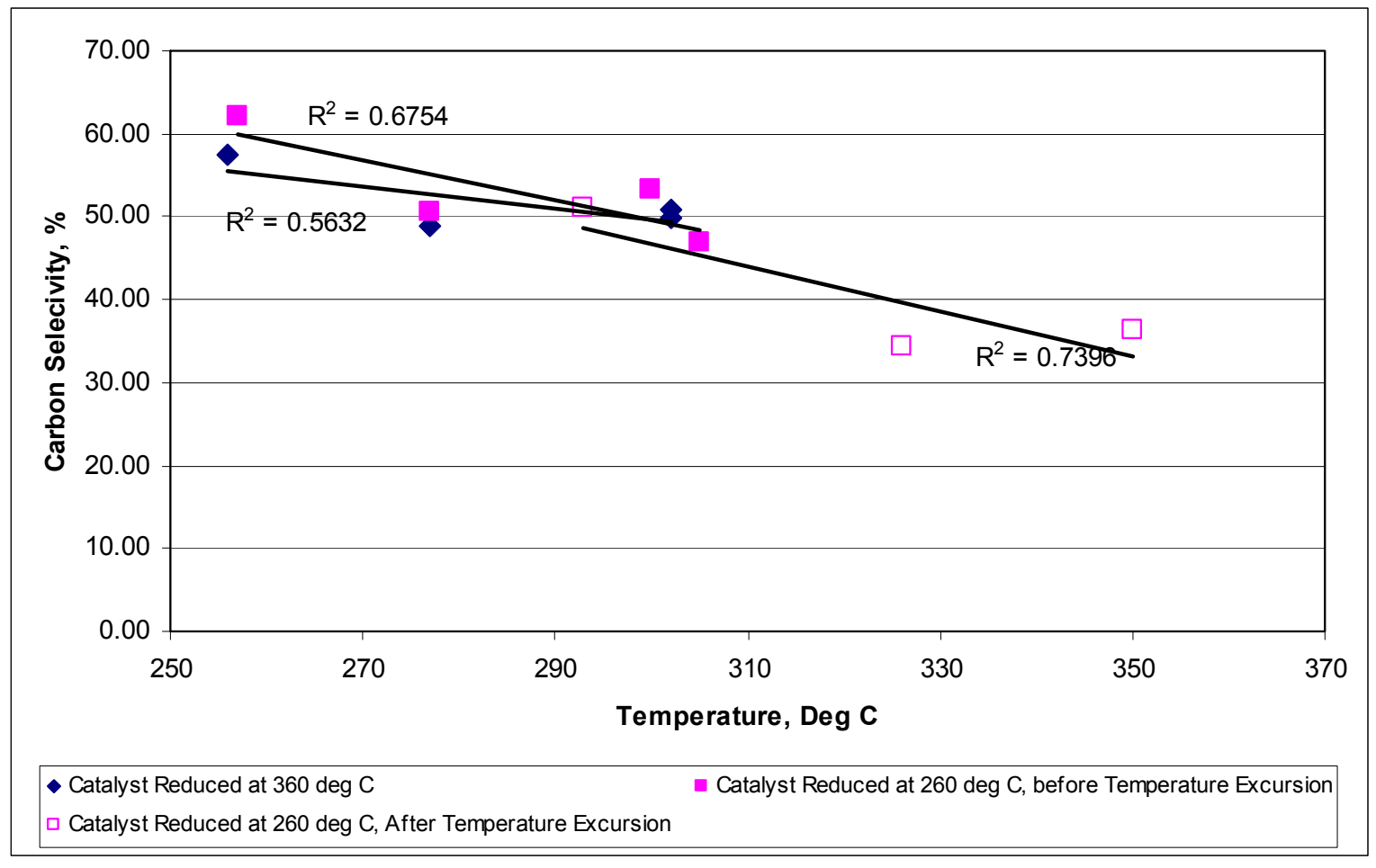

Figure B.11A. Carbon Selectivity to $\mathrm{C}_{2}+$ Oxygenates for Li-Promoted Catalysts

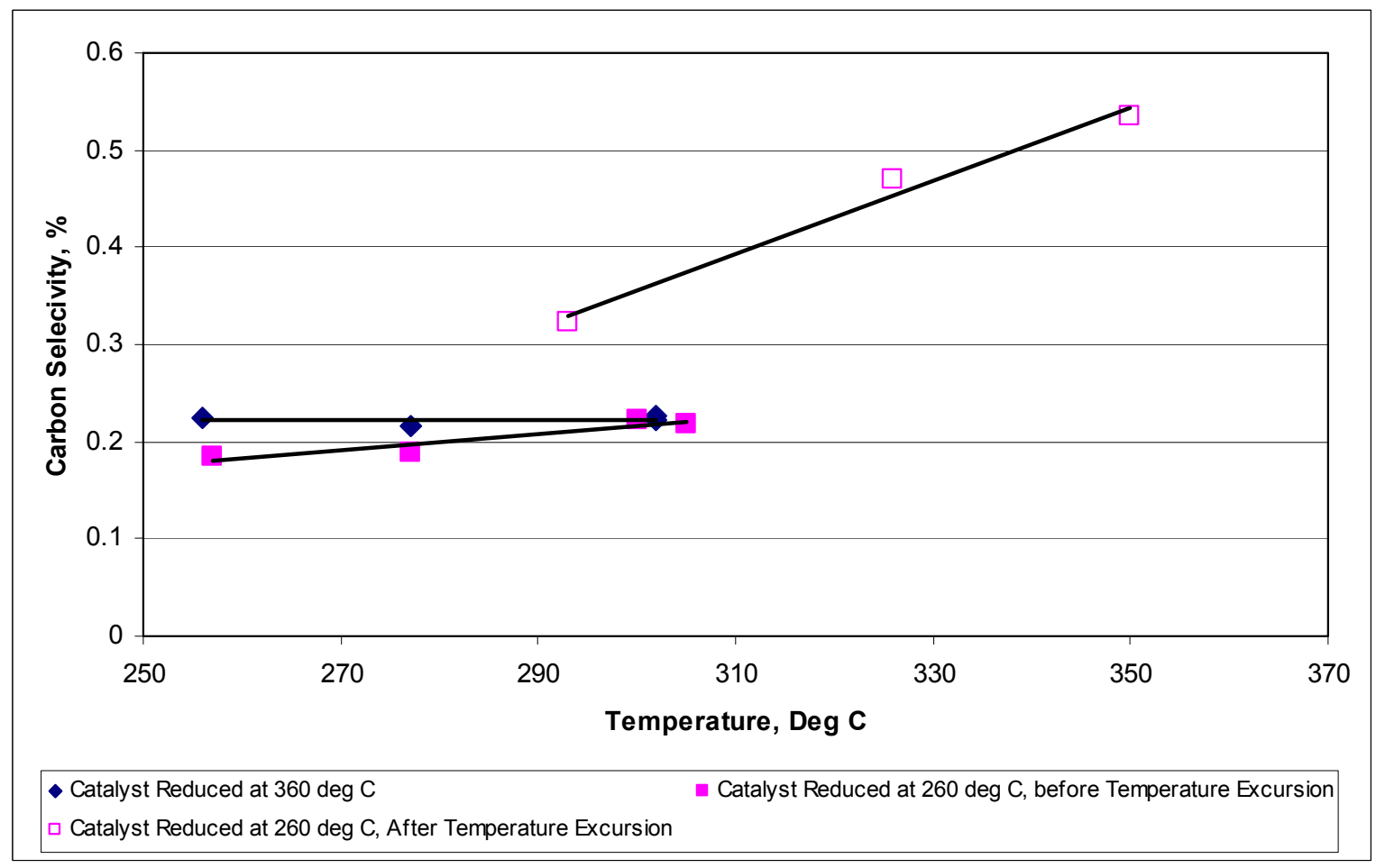

Figure B.12. Carbon Selectivity of All Oxygenates to $\mathrm{C}_{2}+$ Alcohols for Li-Promoted Catalysts 


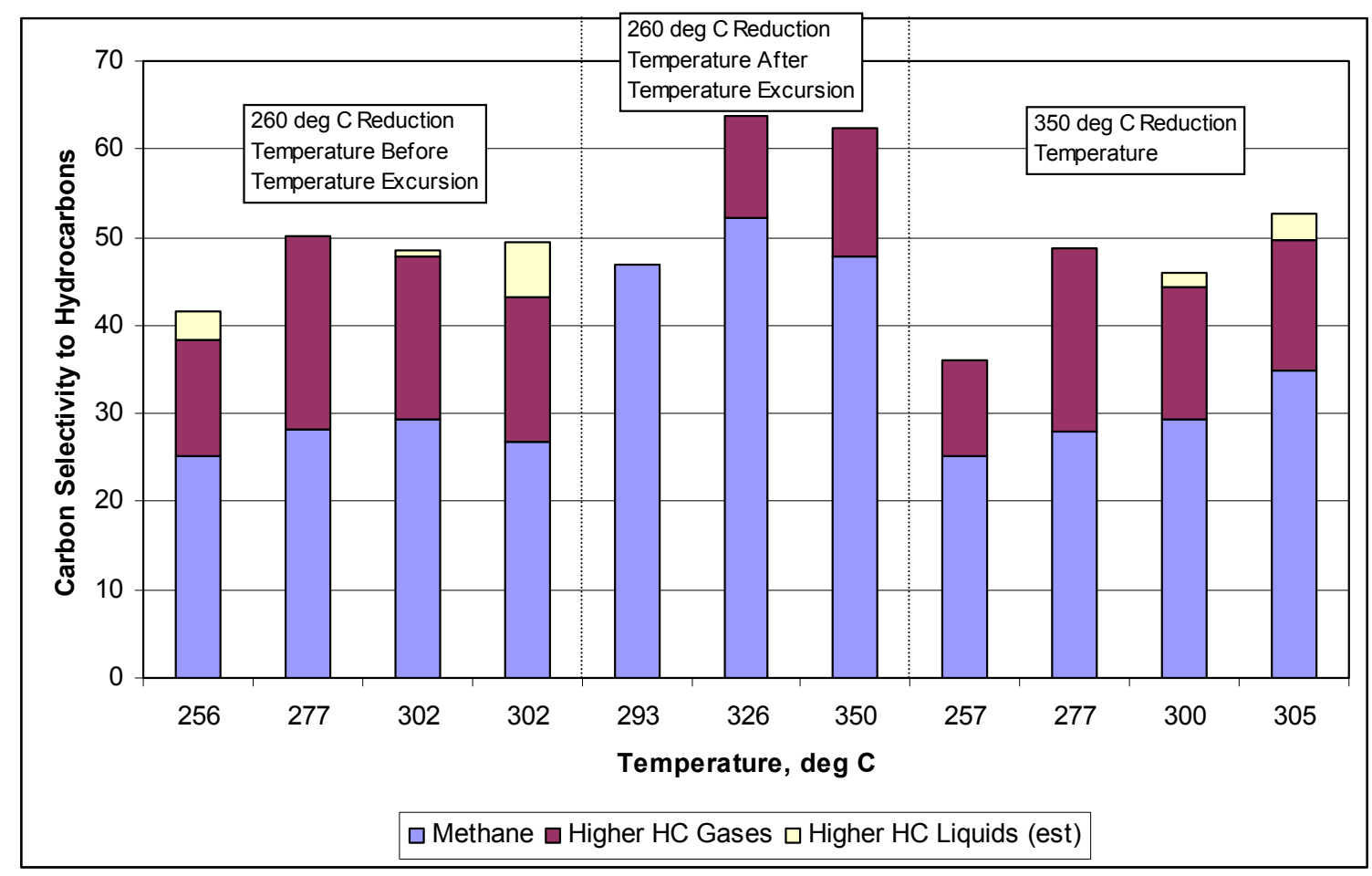

Figure B.13. Comparison of Hydrocarbon Selectivity for Li-Promoted Catalysts

\section{B.4 Ni-Promoted Catalyst}

The Ni-promoted catalyst $\left(\mathrm{RhMnNi} / \mathrm{SiO}_{2}\right)$ was reduced at the maximum temperature of $350^{\circ} \mathrm{C}$ and tested using the furnace to heat the reactor. The testing sequence was 256 and $277^{\circ} \mathrm{C}$ at $7500 \mathrm{~L} / \mathrm{L}_{\text {cat }} / \mathrm{hr}$; 300,305 , and $325^{\circ} \mathrm{C}$ at $11,000 \mathrm{~L} / \mathrm{L}_{\text {cat }} / \mathrm{hr}$; and 312 and $277^{\circ} \mathrm{C}$ at $7500 \mathrm{~L} / \mathrm{L}_{\mathrm{cat}} / \mathrm{hr}$.

It was very difficult to control the catalyst bed temperature at approximately $300^{\circ} \mathrm{C}$ with temperature excursions reaching as high as $350^{\circ} \mathrm{C}$ for a short period of time. The catalyst eventually produced a regular 3-hour cycle between 300 and $335^{\circ} \mathrm{C}$, with a weighted average temperature of approximately $305^{\circ} \mathrm{C}$. Elevating the furnace temperature produced cyclic behavior with a $15^{\circ} \mathrm{C}$ temperature swing between 317 and $332^{\circ} \mathrm{C}$ and a weighted average temperature of approximately $325^{\circ} \mathrm{C}$. Reducing the feed rate by $33 \%$ (same flow rate used for collecting data and samples at approximately 256 and $277^{\circ} \mathrm{C}$ ) while maintaining the same furnace temperature caused the catalyst temperature to decrease to a relatively stable temperature of approximately $312^{\circ} \mathrm{C}$.

Carbon conversion was greater at the lower flow rate as expected as shown in Figure B.14. It also appears that some catalyst deactivation occurred over the testing time as indicated by the decrease in carbon conversion from approximately 17 to $12 \%$ at $277^{\circ} \mathrm{C}$. The catalyst showed similar behavior in regards to the $\mathrm{C}_{2}+$-oxygenate STYs as shown in Figure B.15. This is distinctly different from previously tested catalysts for which the space velocity did not have a significant effect on the $\mathrm{C}_{2}{ }^{+ \text {-oxygenate STYs }}$ for the space velocity/temperature combinations tested.

The effects of space velocity on the carbon selectivity to $\mathrm{C}_{2}+$ oxygenates and to $\mathrm{C}_{2}+$ alcohols are much less pronounced as is shown in figures B.16 and B.27. However, the trends of decreasing carbon selectivity to $\mathrm{C}_{2}+$ oxygenates and increasing carbon selectivity of all oxygenates to $\mathrm{C}_{2}+$ alcohols with 
increasing temperature are consistent with the behavior of the previously tested catalysts. Furthermore, it appears that carbon selectivity of all oxygenates to $\mathrm{C}_{2}+$ alcohols increased during catalyst deactivation at $277^{\circ} \mathrm{C}$ as shown in figure B. 17 , where the carbon selectivity to $\mathrm{C}_{2}+$ alcohols increased from 17 to $26 \%$.

Figure B.18 compares the carbon selectivity to hydrocarbons for the Ni-promoted catalyst. It appears that a regular increase in hydrocarbon selectivity with increasing temperature occurred up to about $300^{\circ} \mathrm{C}$, but then decreased over time while cycling between 300 and $335^{\circ} \mathrm{C}$ during sample acquisition. Subsequent test conditions at 325 and $277^{\circ} \mathrm{C}$ indicate that selectivity to hydrocarbons had decreased because of catalyst deactivation during the temperature excursions. There also appeared to be a shift in carbon selectivity away from higher hydrocarbons to methane during catalyst deactivation.

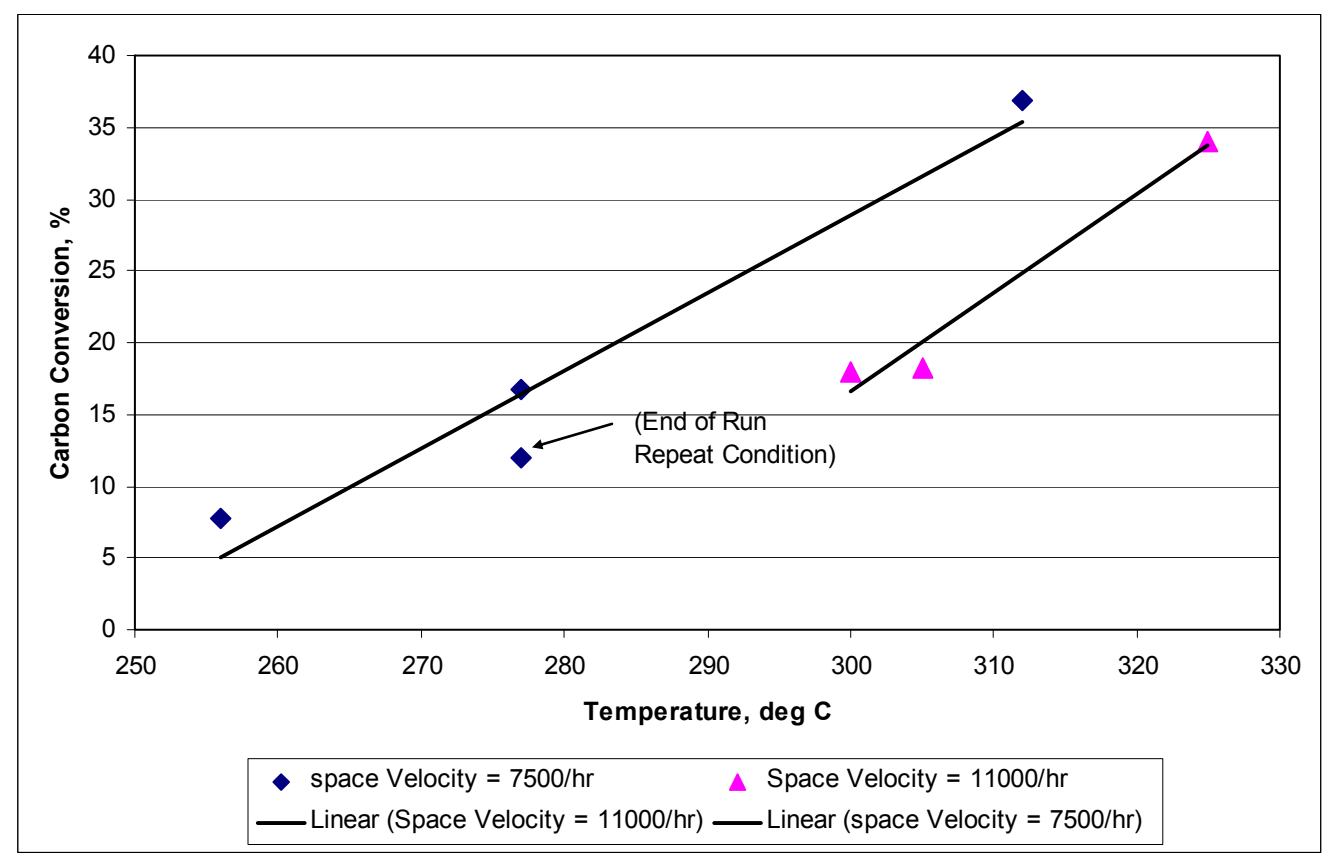

Figure B.14. Carbon Conversion for Ni-Promoted Catalyst 


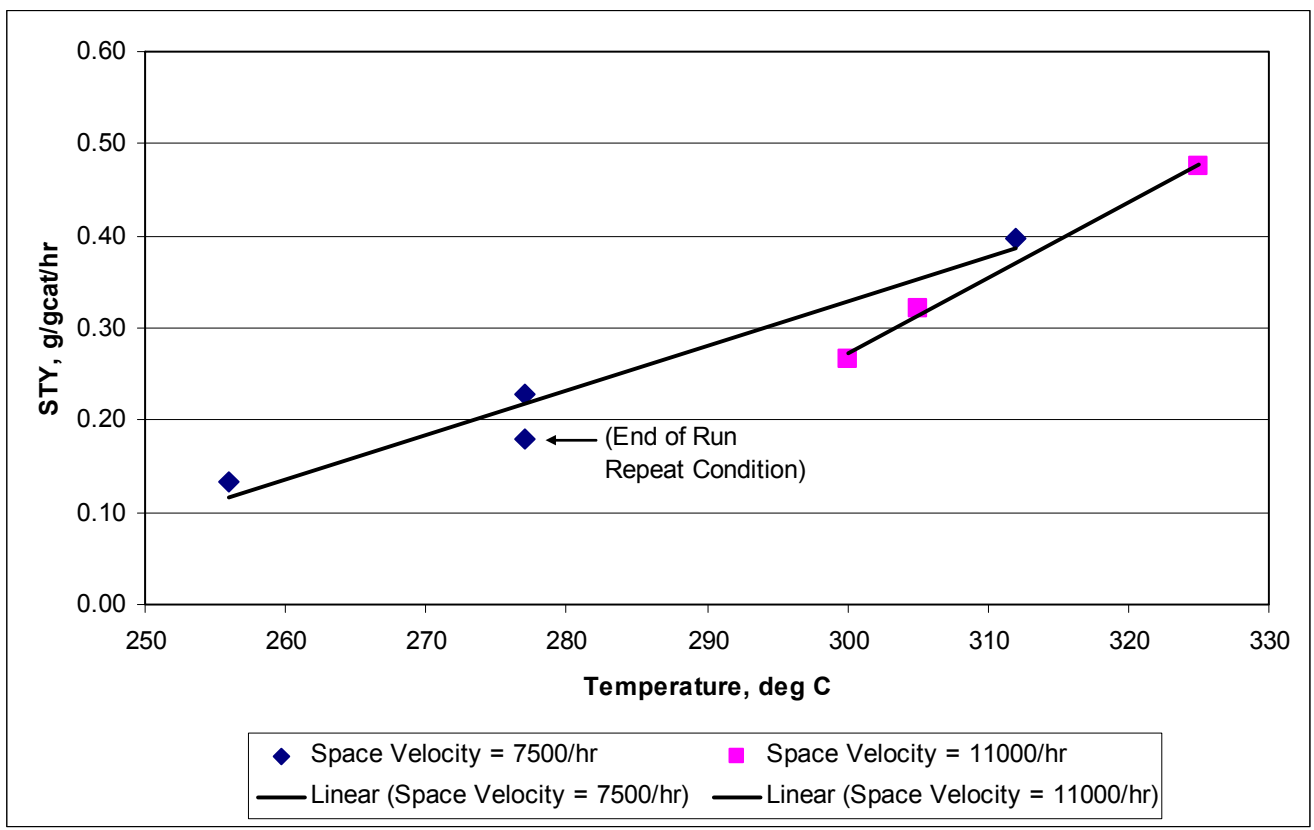

Figure B.15. $\mathrm{C}_{2}+-$ Oxygenate STYs for Ni-Promoted Catalyst

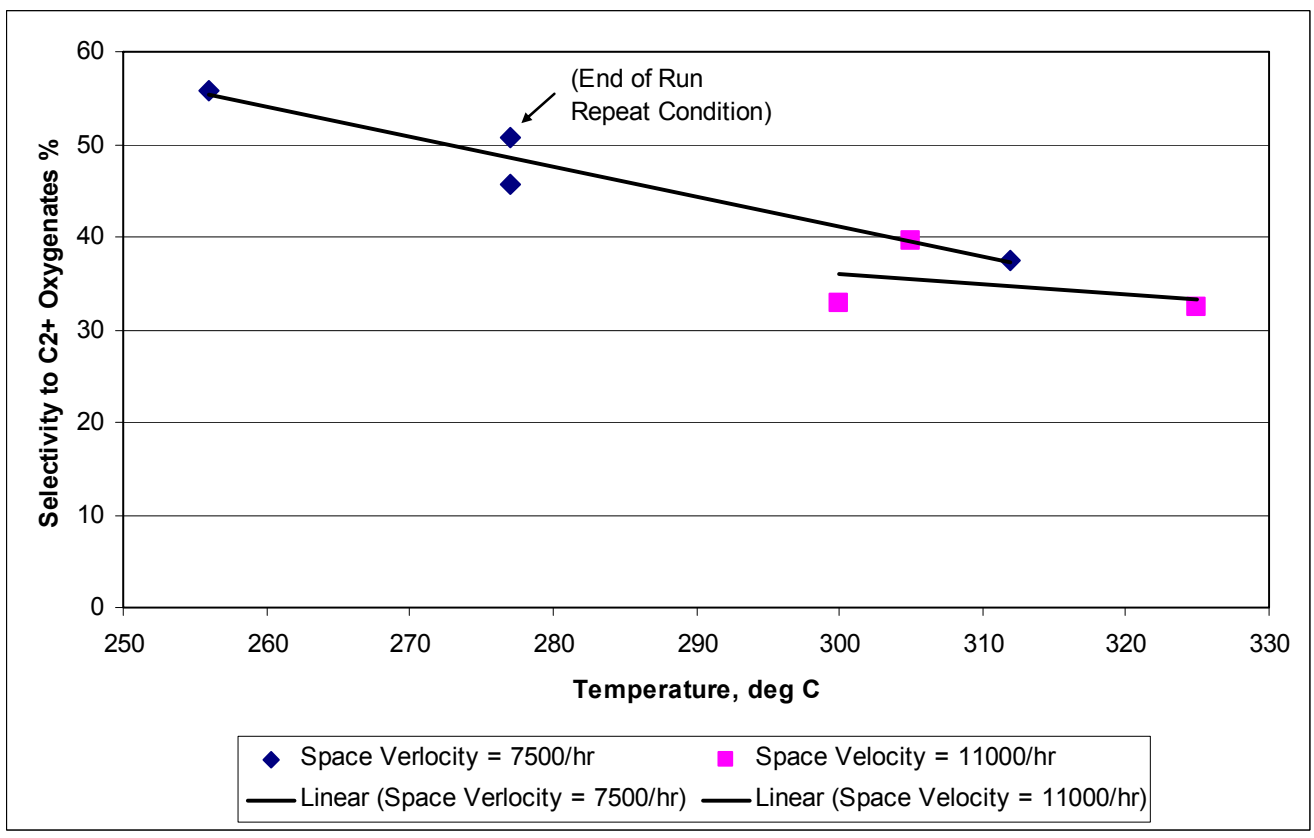

Figure B.16. Converted Carbon Selectivity to $\mathrm{C}_{2}+$ Oxygenates for Ni-Promoted Catalyst 


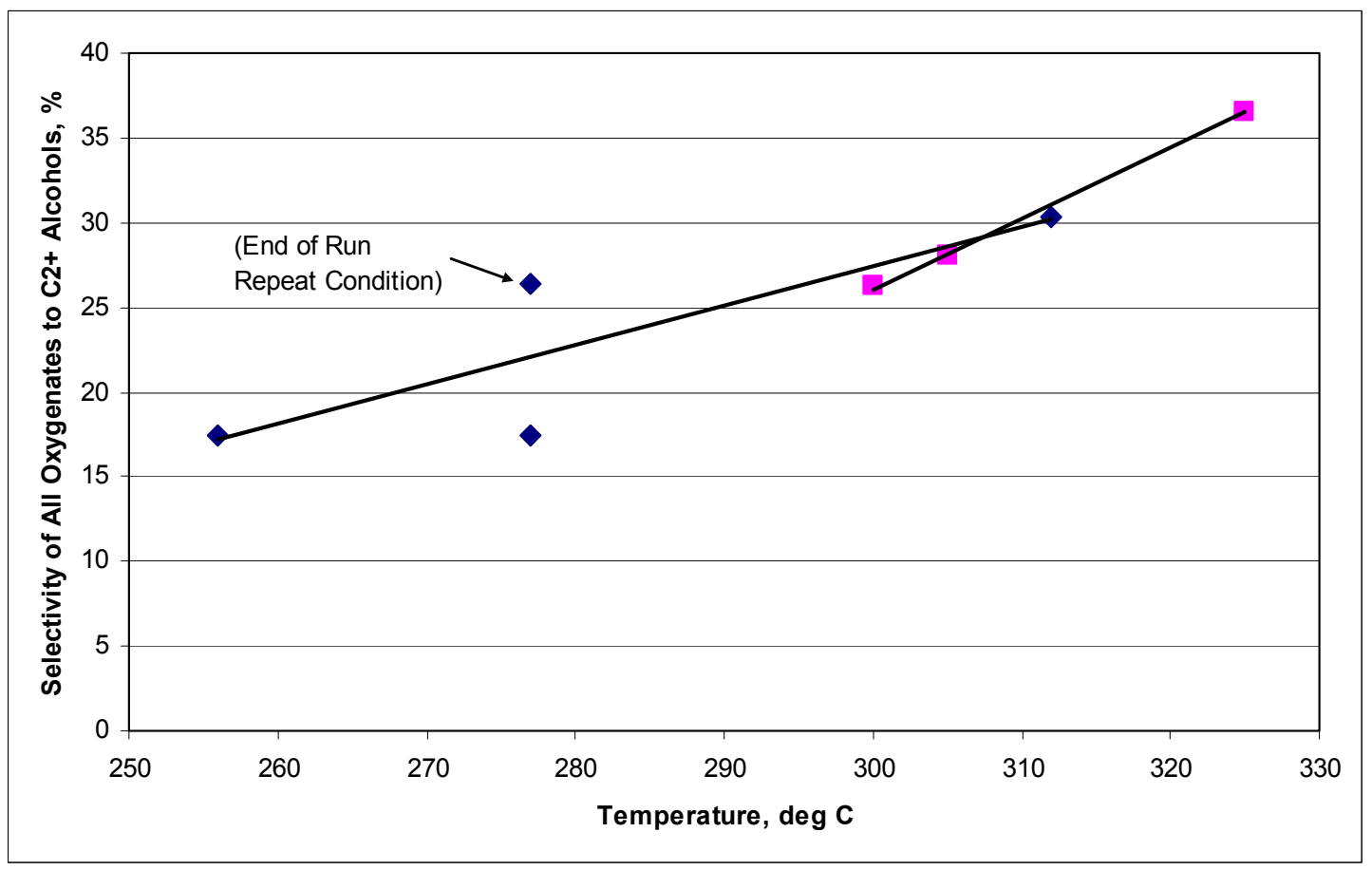

Figure B.17. Carbon Selectivity of All Oxygenates to $\mathrm{C}_{2}+$ Alcohols for Ni-Promoted Catalyst

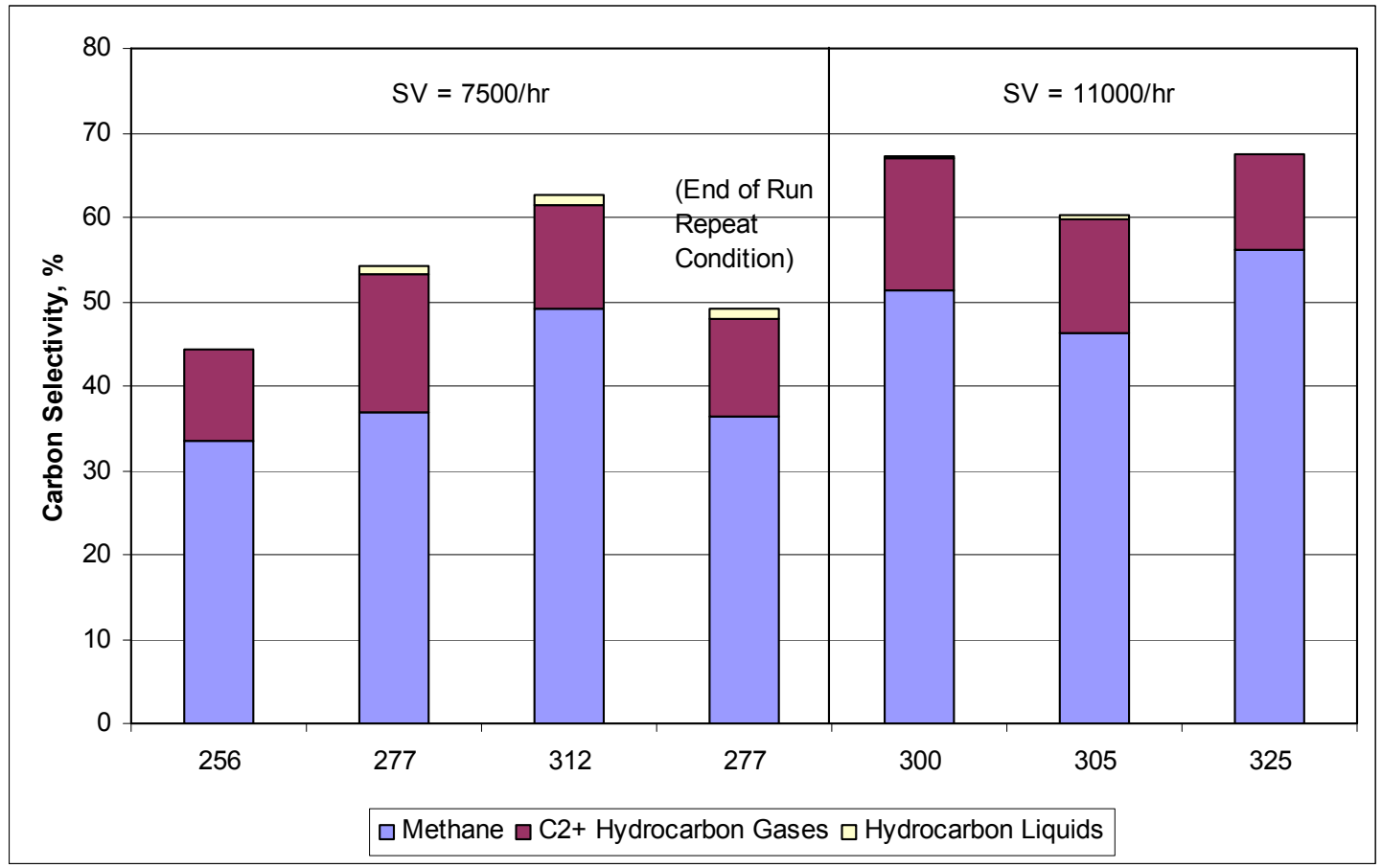

Figure B.18. Comparison of Hydrocarbon Selectivity for Ni-Promoted Catalyst 


\section{B.5 Re-Promoted Catalyst}

The Re-promoted catalyst $\left(\mathrm{RhMnRe} / \mathrm{SiO}_{2}\right)$ was reduced at the maximum temperature of $350^{\circ} \mathrm{C}$ and tested using the furnace to heat the reactor. The testing sequence was 256 and $273^{\circ} \mathrm{C}$ at $7500 \mathrm{~L} / \mathrm{L}_{\text {cat }} / \mathrm{hr}$ and $325^{\circ} \mathrm{C}$ at $11,000 \mathrm{~L} / \mathrm{L}_{\text {cat }} / \mathrm{hr}$.

It was not possible to control the catalyst bed temperature at approximately $300^{\circ} \mathrm{C}$ using the furnace, with temperature excursions reaching as high as $350^{\circ} \mathrm{C}$ for a short period of time on one occasion and ranging between 275 and $325^{\circ} \mathrm{C}$ in response to small changes in the furnace temperature. An attempt was made to set conditions at $325^{\circ} \mathrm{C}$, but again, it was difficult to maintain a stable temperature. Temperature slowly dropped from approximately $333^{\circ} \mathrm{C}$ to approximately $325^{\circ} \mathrm{C}$ over 11 hours before abruptly dropping to $288^{\circ} \mathrm{C}$, and slowly increasing to $290^{\circ} \mathrm{C}$ over about a 4-hour period. The temperature was elevated with the furnace to approximately $325^{\circ} \mathrm{C}$ and held there for about 5 hours, at which time a sample was taken. The weighted average temperature during sample collection period was estimated to be $324^{\circ} \mathrm{C}$. While the conditions experienced by the catalyst while collecting the sample were not very good, the data was collected to provide an indication of catalyst performance.

Carbon conversion at $324^{\circ} \mathrm{C}$, as shown in Figure B.19, appears to be lower than an extrapolated value based on the carbon conversions at 256 and $273^{\circ} \mathrm{C}$, and could be accounted for, at least in part, by the higher space velocity used during the test at $324^{\circ} \mathrm{C}$. The catalyst also appears to show a lower $\mathrm{C}_{2}+-$ oxygenate $\mathrm{STY}$ at $325^{\circ} \mathrm{C}$ than an extrapolated value based on the carbon conversions at 256 and $273^{\circ} \mathrm{C}$ as shown in Figure B.20. There is insufficient data to speculate on the cause. However, even the extrapolated value to $325^{\circ} \mathrm{C}$, based on the performance of the catalyst at the lower temperatures, suggests that the Re-promoted catalyst would not likely achieve a $\mathrm{C}_{2}+$-oxygenate STY greater than about $350 \mathrm{~g} / \mathrm{L}_{\text {cat }} / \mathrm{hr}$.

Carbon selectivity to $\mathrm{C}_{2}+$ oxygenates decreased with increasing temperature, as shown in Figure B.21, which is consistent with the other catalysts. The catalyst also showed an increasing carbon selectivity of $\mathrm{C}_{2}+$ oxygenates to $\mathrm{C}_{2}+$ alcohols with increasing temperature as shown in Figure B.22, reaching a maximum selectivity ratio of 0.60 at $325^{\circ} \mathrm{C}$. Figure B. 23 shows that the carbon selectivity to hydrocarbon liquids and $\mathrm{C}_{2}+$ hydrocarbon gases decreased at temperatures above $273^{\circ} \mathrm{C}$, which is similar to the behavior of the other catalysts. It should be noted that carbon selectivity to hydrocarbons was very high even at the lowest temperatures tested. 


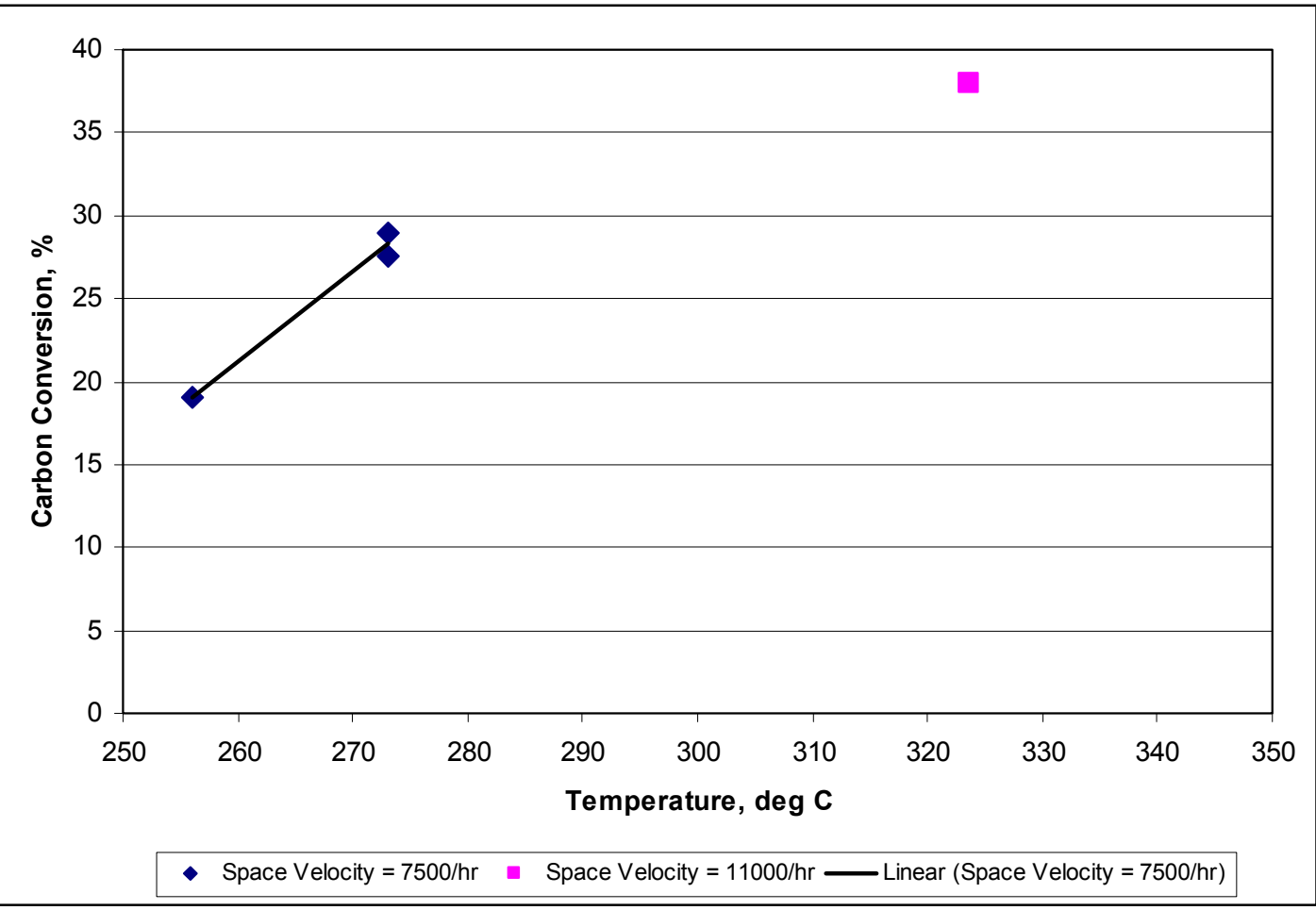

Figure B.19. Carbon Conversion for the Re-Promoted Catalyst

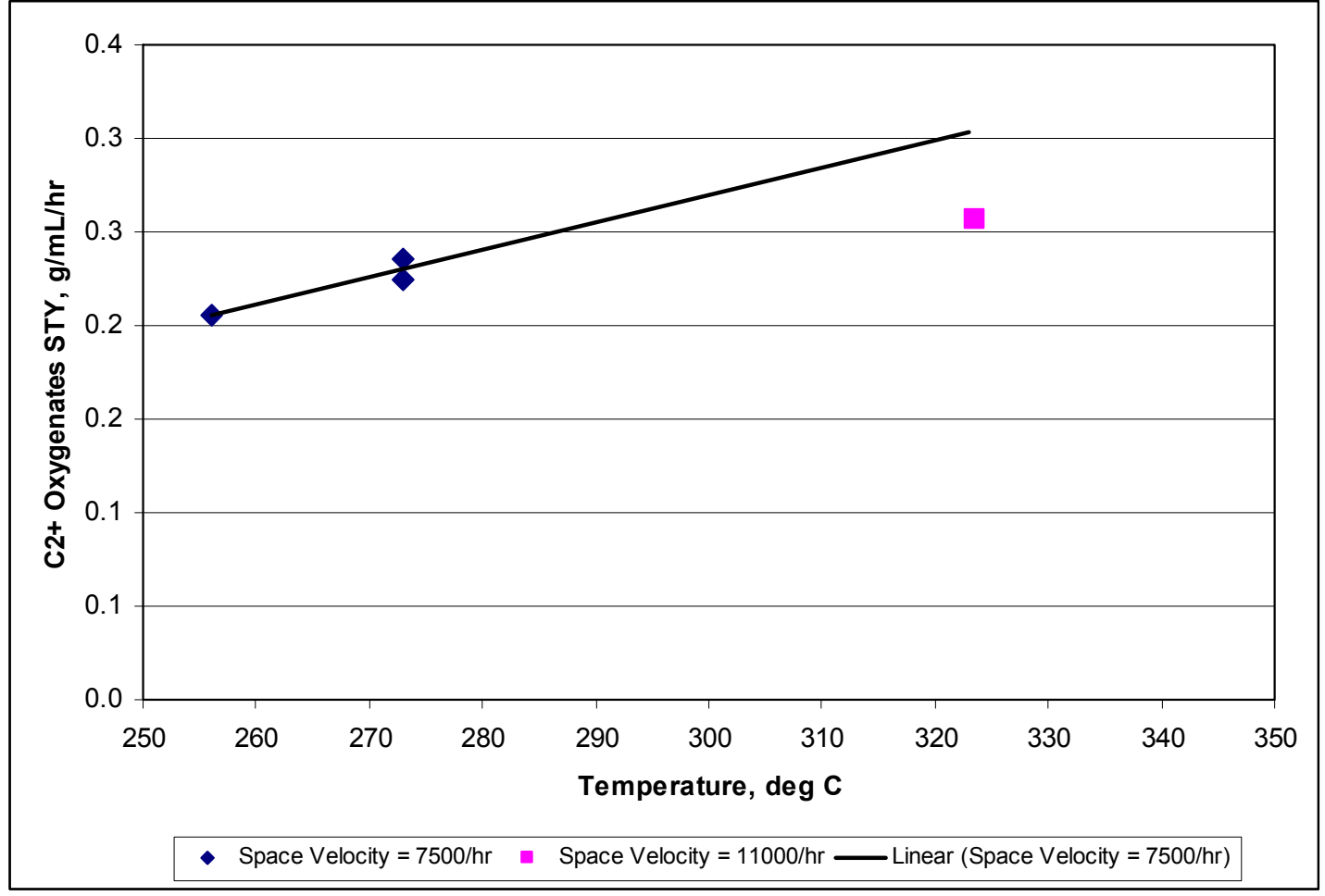

Figure B.20. $\mathrm{C}_{2}+-$ Oxygenate STYs for the Re-Promoted Catalyst 


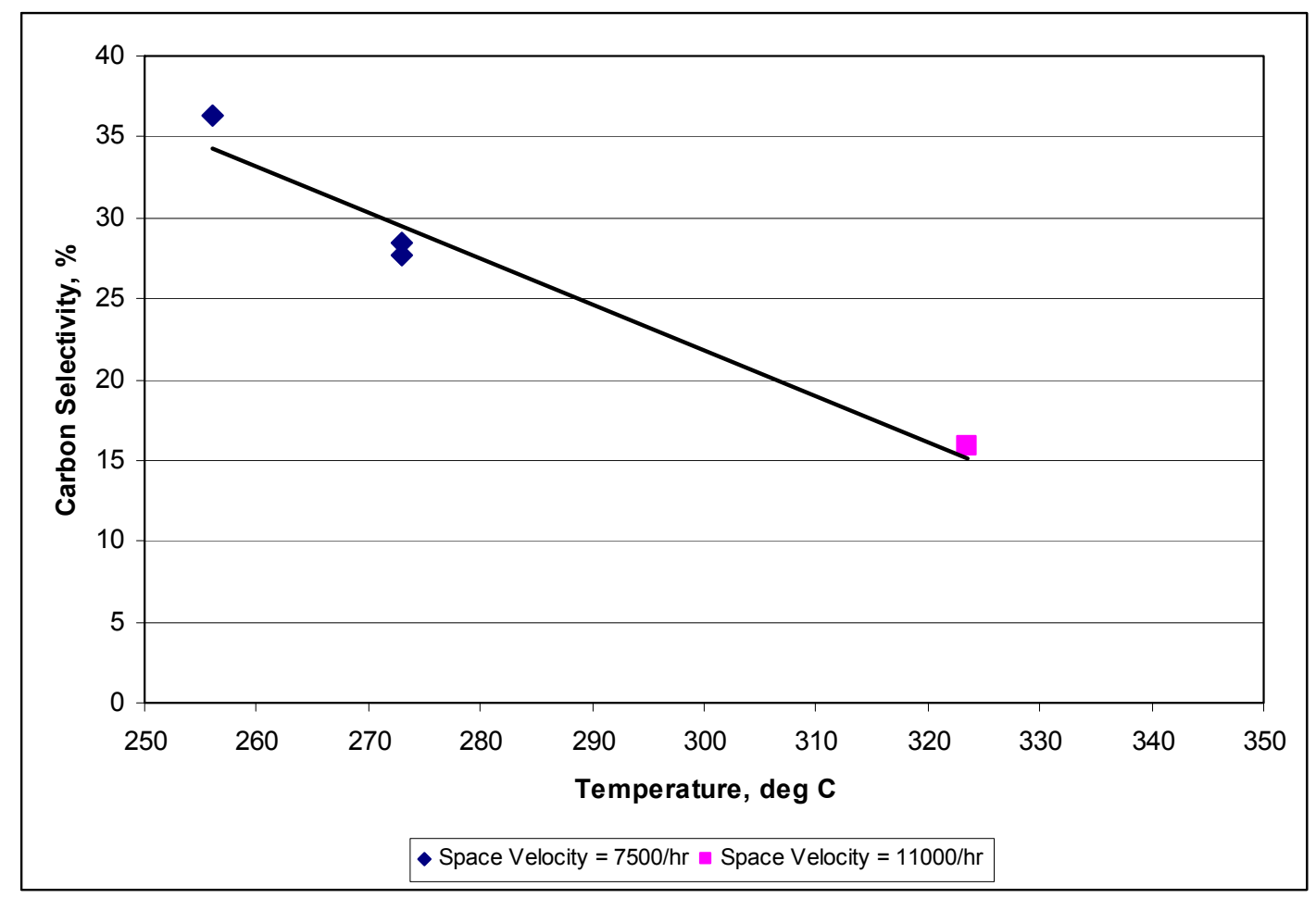

Figure B.21. Converted Carbon Selectivity to $\mathrm{C}_{2}+$ Oxygenates for the Re-Promoted Catalyst

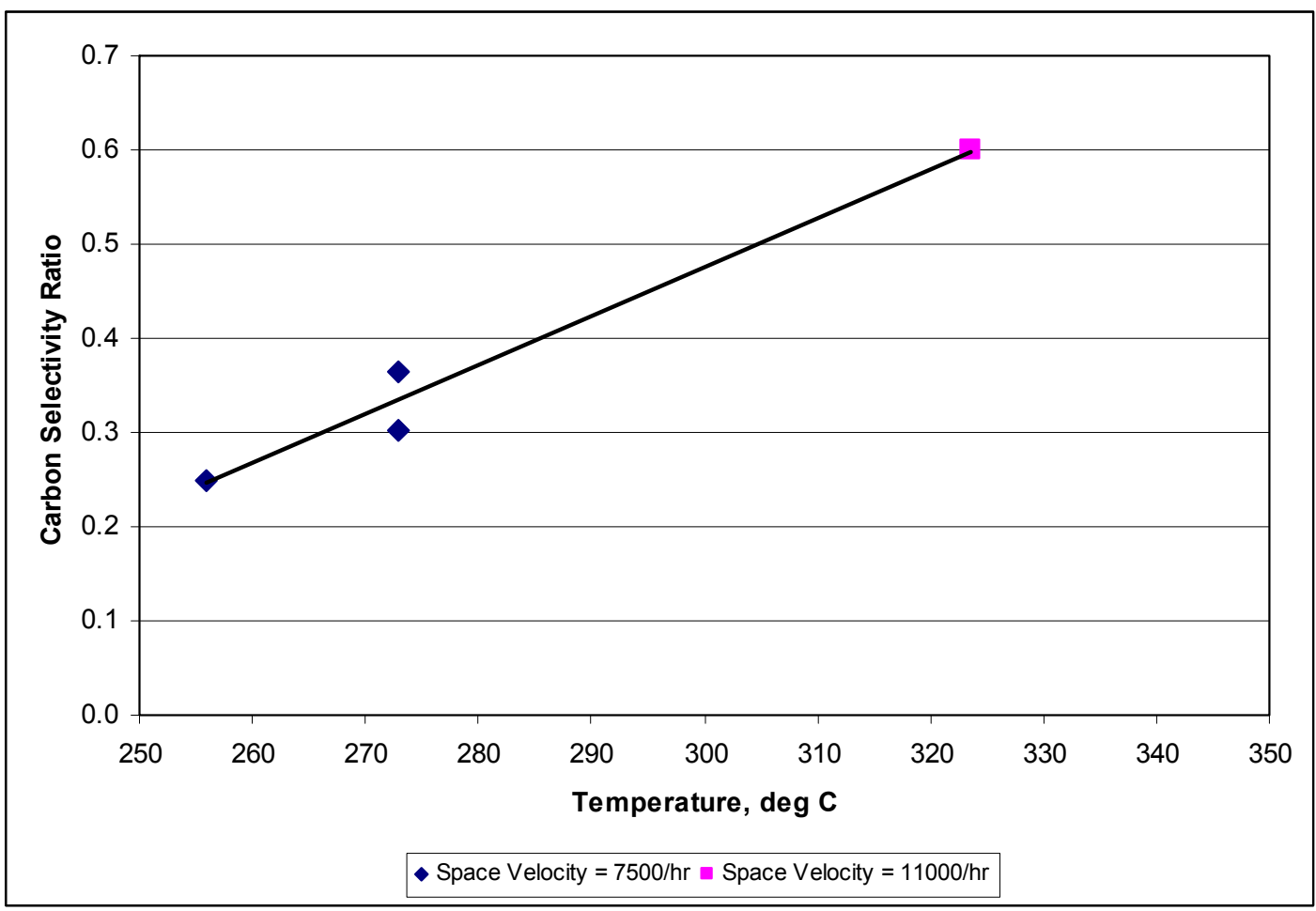

Figure B.22. Carbon Selectivity of All Oxygenates to $\mathrm{C}_{2}+$ Alcohols for the Re-Promoted Catalyst 


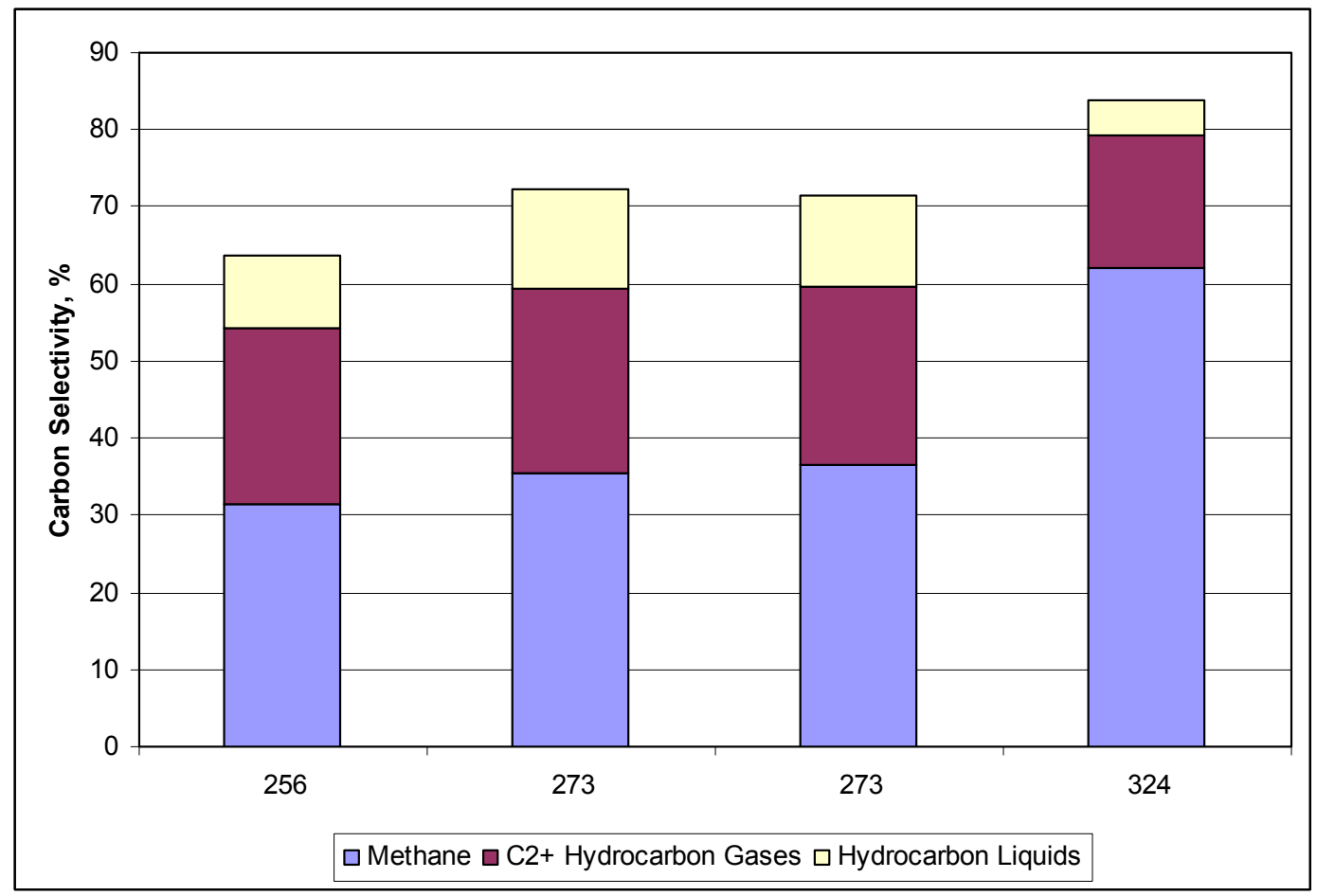

Figure B.23. Comparison of Hydrocarbon Selectivity for the Re-Promoted Catalyst

\section{B.6 Ir-Promoted Catalyst}

Two tests were conducted with the Ir-promoted catalyst ( $\mathrm{RhMnIr} / \mathrm{SiO}{ }_{2}$ and $\mathrm{RhMnIr} / \mathrm{SiO}_{2} \mathrm{~A}$ catalysts) to compare the catalyst performance using both the furnace and the hot oil circulating system to control the catalyst temperature. The testing sequence for the first test was 256 and $267^{\circ} \mathrm{C}$ at $7500 \mathrm{~L} / \mathrm{L}_{\text {cat }} / \mathrm{hr}$, and $328^{\circ} \mathrm{C}$ at $11,000 \mathrm{~L} / \mathrm{L}_{\text {cat }} / \mathrm{hr}$. The testing sequence for the second test was 256 and $276^{\circ} \mathrm{C}$ at $7500 \mathrm{~L} / \mathrm{L}_{\text {cat }} / \mathrm{hr} ; 303,316$, and $323^{\circ} \mathrm{C}$ at $11,000 \mathrm{~L} / \mathrm{L}_{\text {cat }} / \mathrm{hr} ; 325^{\circ} \mathrm{C}$ at $15,000 \mathrm{~L} / \mathrm{L}_{\text {cat }} / \mathrm{hr}$; and 303 and $304^{\circ} \mathrm{C}$ at $11,000 \mathrm{~L} / \mathrm{L}_{\text {cat }} / \mathrm{hr}$.

During the first test using the furnace, it was very difficult to obtain a stable temperature at approximately $275^{\circ} \mathrm{C}$, so data a samples were collected over a period during which the temperature fluctuated between 262 and $278^{\circ} \mathrm{C}$ for a weighted average temperature of $267^{\circ} \mathrm{C}$. The catalyst temperature during the next sample collection period ranged from 300 to $334^{\circ} \mathrm{C}$ with a weighted average temperature of $328^{\circ} \mathrm{C}$.

During the second test, catalyst temperature control was very improved, with catalyst temperatures generally staying within $\pm 2{ }^{\circ} \mathrm{C}$ (with the exception of the $303^{\circ} \mathrm{C}$ condition, which varied from 293 to $308^{\circ} \mathrm{C}$ for about $10 \%$ of the sample collection time, 298 to $305^{\circ} \mathrm{C}$ for about a third of the time, and between 302 and $305^{\circ} \mathrm{C}$ for the remainder of the time as the power output of the oil heater was reduced to reduce the cyclic temperature range of the heater).

Figure B.24 shows the carbon conversion for the various conditions of the two tests. It can be seen that the carbon conversion decreases with increasing space velocity as might be expected. It also appears 
that the carbon conversions for the test using the hot oil circulating system were slightly lower for the same catalyst temperatures. Furthermore it appears from the repeated conditions that the catalyst deactivated slightly during the test, possibly because of temperatures that exceeded $300^{\circ} \mathrm{C}$.

Figure B.25 shows the $\mathrm{C}_{2}+$-oxygenate STYs for the two tests. It appears that the $\mathrm{C}_{2}+$-oxygenate STYs peaked between $316^{\circ} \mathrm{C}$ (approximately $690 \mathrm{~g} / \mathrm{L}_{\text {cat }} / \mathrm{hr}$ ) and $323^{\circ} \mathrm{C}$ (approximately $600 \mathrm{~g} / \mathrm{L}_{\text {cat }} / \mathrm{hr}$ ) for a space velocity of $11,000 \mathrm{~L} / \mathrm{L}_{\text {cat }} / \mathrm{hr}$ in the second test using the hot oil circulating system. Furthermore, the $\mathrm{C}_{2}+\mathrm{STY}$ for the highest temperature in the first test, using the furnace, continued the downward STY trend at a higher catalyst temperature (approximately $500 \mathrm{~g} / \mathrm{L}_{\text {cat }} / \mathrm{hr}$ at $328^{\circ} \mathrm{C}$ ). Increasing the space velocity to $15,000 \mathrm{~L} / \mathrm{L}_{\text {cat }} / \mathrm{hr}$ in the second test resulted in $\mathrm{C}_{2}+\mathrm{STY}$ of approximately $810 \mathrm{~g} / \mathrm{L}_{\text {cat }} / \mathrm{hr}$ at $325^{\circ} \mathrm{C}$. It also appears that the $\mathrm{C}_{2}+\mathrm{STY}$ decreased because of high temperature deactivation as indicated by the STYs for the repeated tests.

Carbon selectivity to $\mathrm{C}_{2}+$ oxygenates decreased with increasing temperature, as shown in Figure B.26, which is consistent with the other catalysts. The catalyst also showed an increasing carbon selectivity of $\mathrm{C}_{2}+$ oxygenates to $\mathrm{C}_{2}+$ alcohols with increasing temperature as shown in Figure B.27. There also appeared to be more scatter in the data with respect to these trends for the first test using the furnace for heating, probably in part because of the difficulty in maintaining the catalyst temperature in these tests.

Figure B.28 compares the carbon selectivity to the hydrocarbons during both tests. It can be seen that the selectivity to the hydrocarbon liquids decreased with increasing temperature once the catalyst temperature exceeded $267^{\circ} \mathrm{C}$. The repeat conditions of the second test at approximately $303^{\circ} \mathrm{C}$ also suggest that there was a decrease in the selectivity to the $\mathrm{C}_{2}+$ hydrocarbon gases that can be attributed to catalyst deactivation at higher temperatures. 


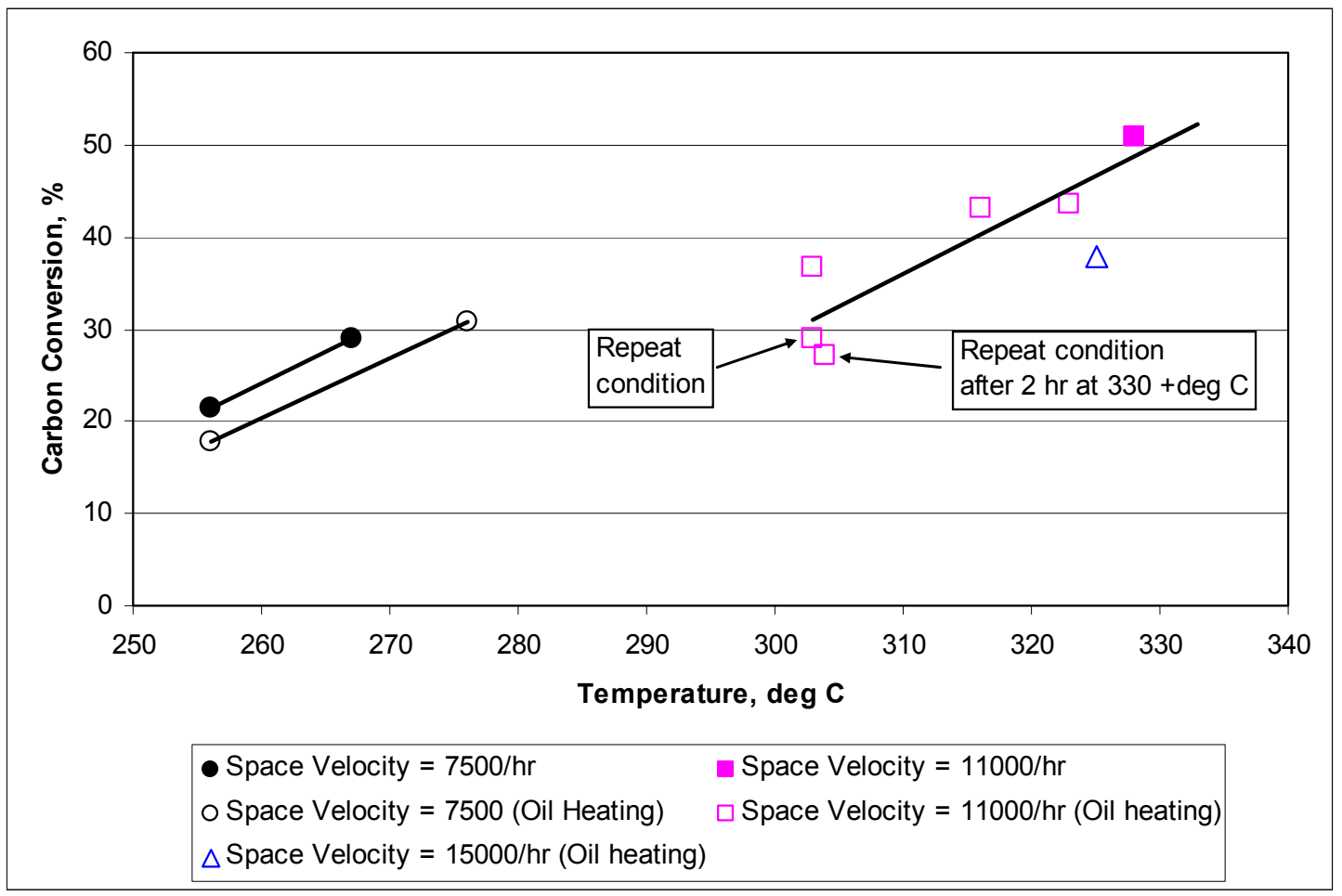

Figure B.24. Carbon Conversion for the Ir-Promoted Catalyst

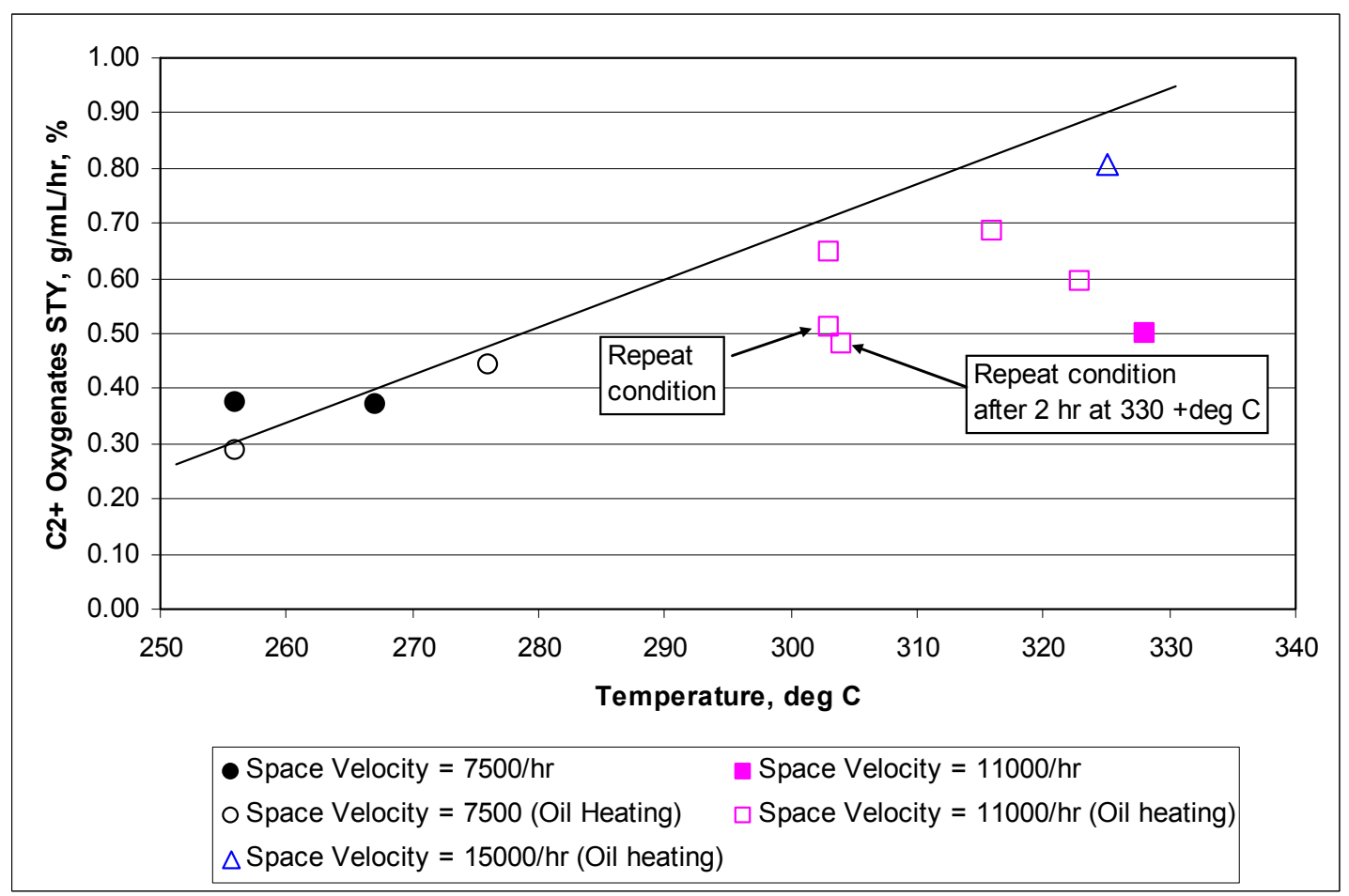

Figure B.25. $\mathrm{C}_{2}+-$ Oxygenate STYs for the Ir-Promoted Catalyst 


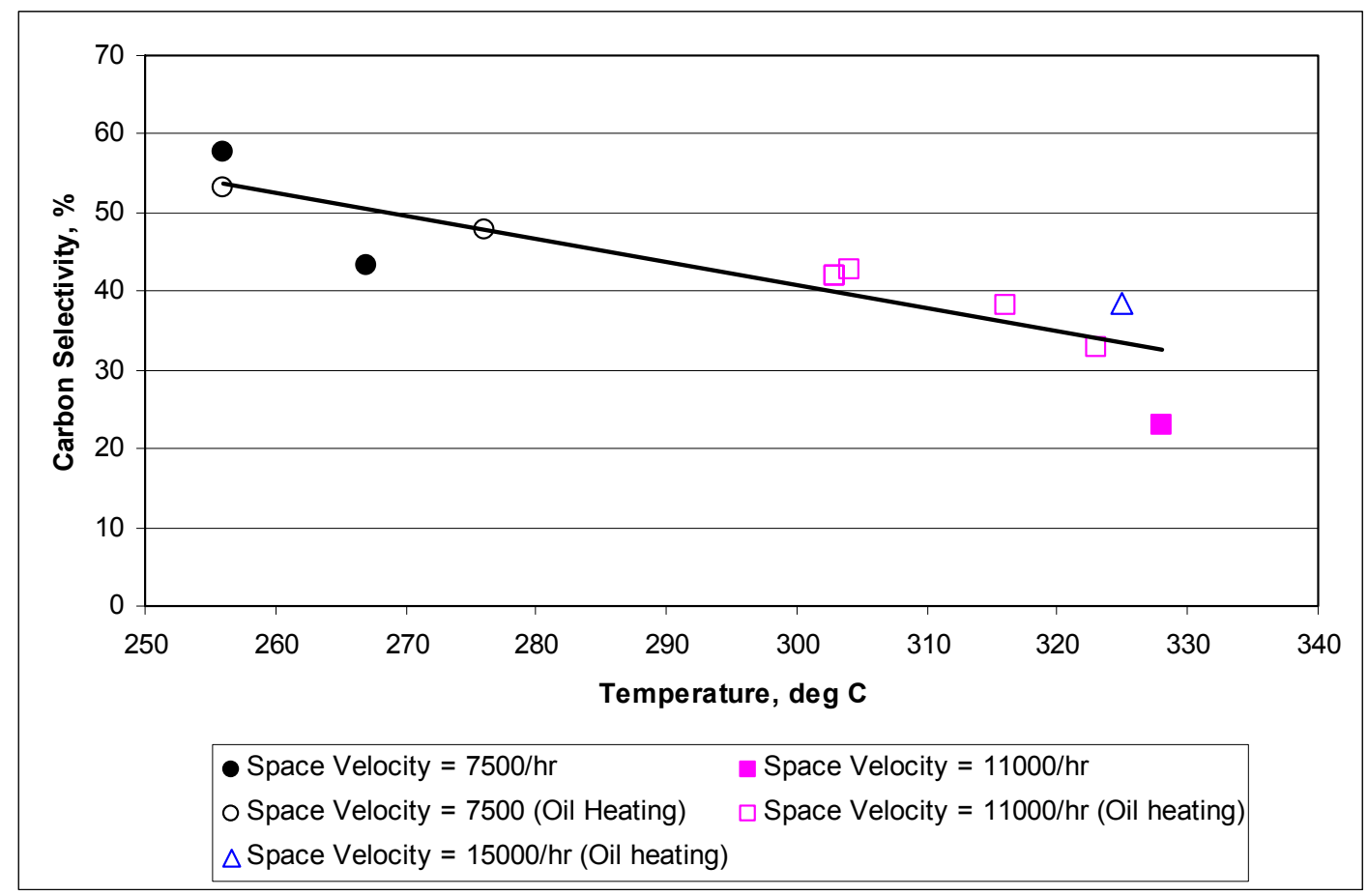

Figure B.26. Converted Carbon Selectivity to $\mathrm{C}_{2}+$ Oxygenates for the Ir-Promoted Catalyst

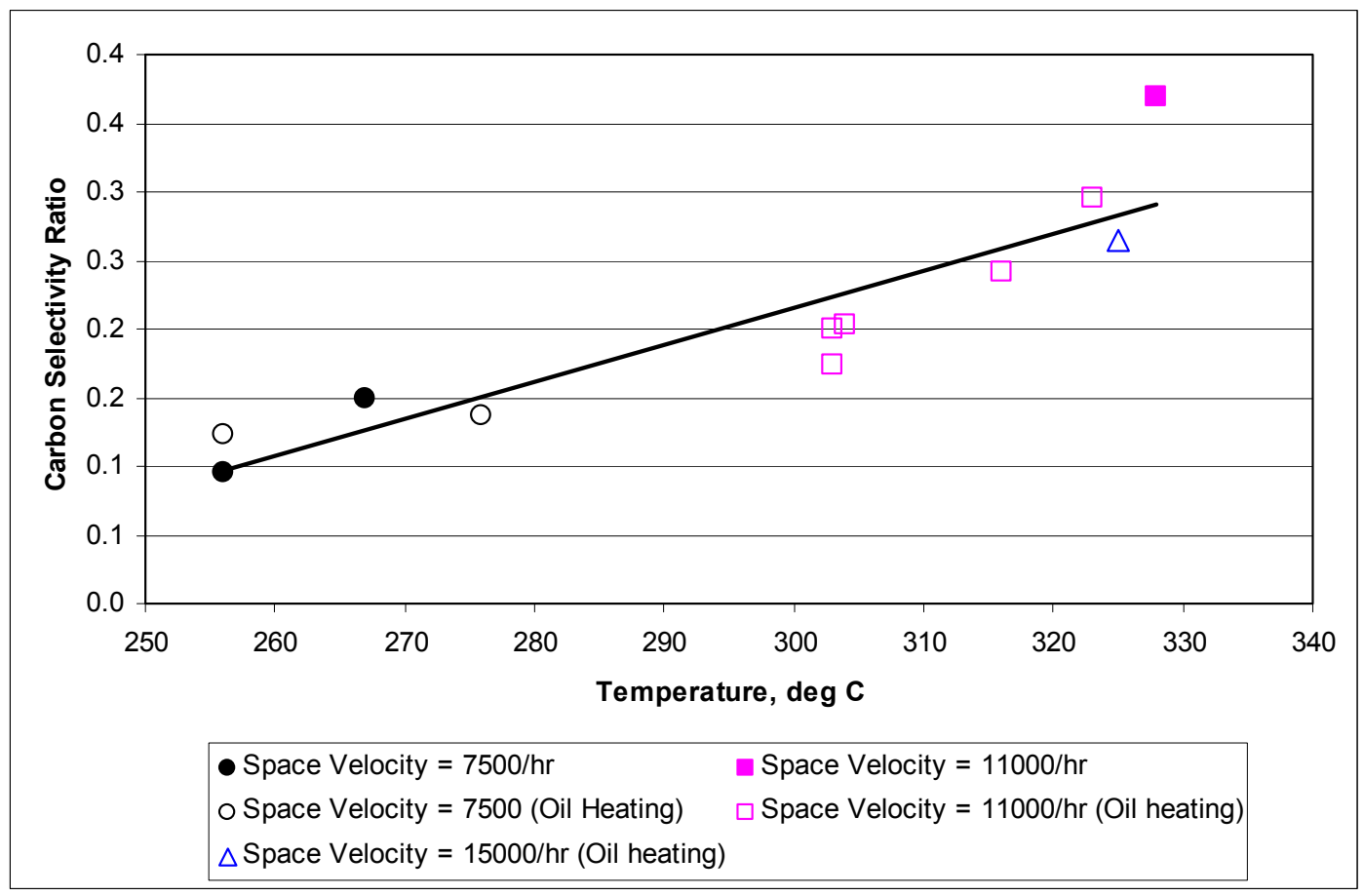

Figure B.27. Carbon Selectivity of All Oxygenates to $\mathrm{C}_{2}+$ Alcohols for the Ir-Promoted Catalyst 


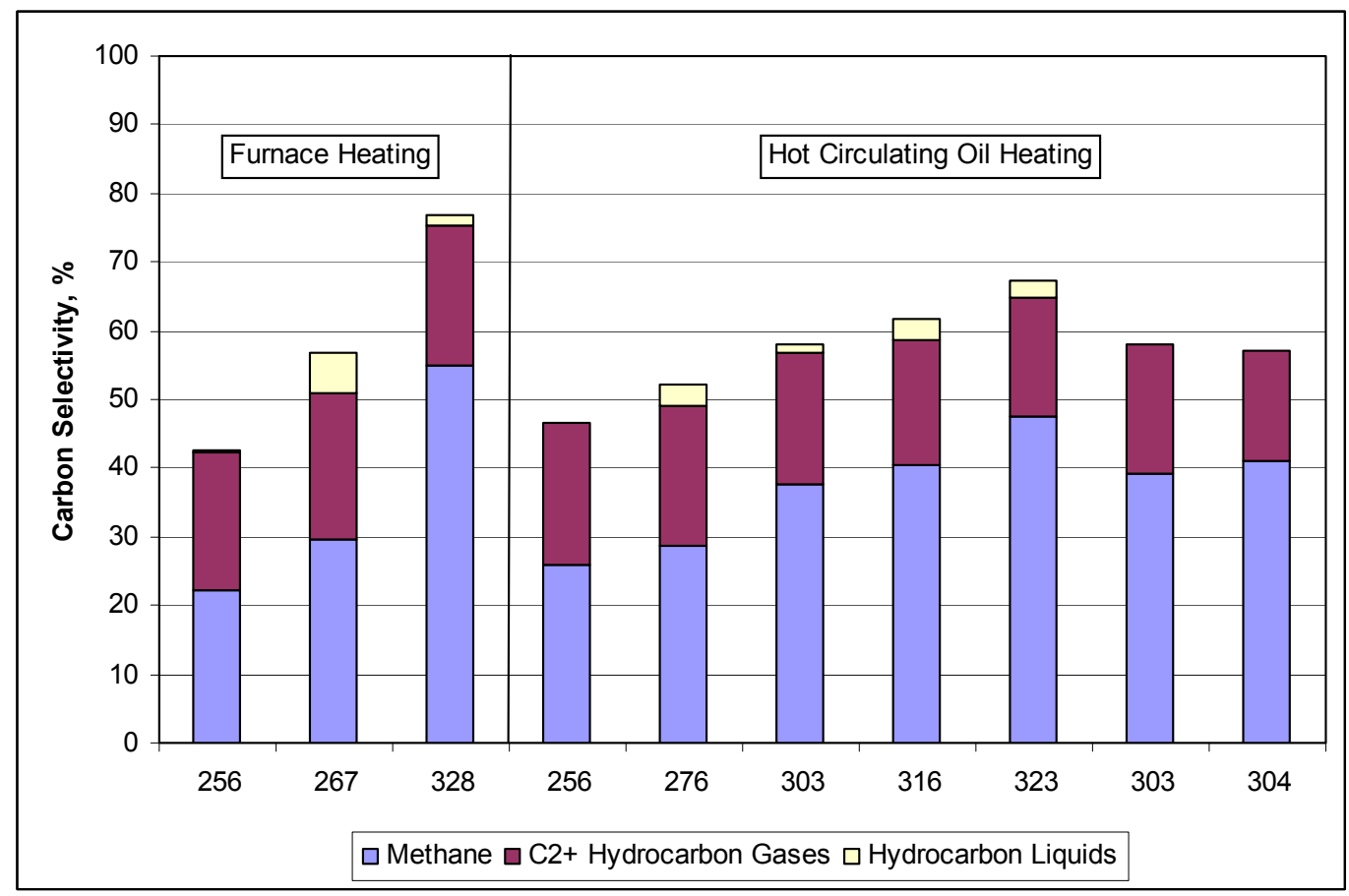

Figure B.28. Comparison of Hydrocarbon Selectivities for the Ir-Promoted Catalyst

\section{B.7 Cu-Promoted Catalyst}

The Cu-promoted catalyst $\left(\mathrm{RhMnCu} / \mathrm{SiO}_{2}\right)$ was reduced at the maximum temperature of $350^{\circ} \mathrm{C}$ and tested using the hot oil circulating system to heat the reactor. The testing sequence was 256 and $276^{\circ} \mathrm{C}$ at $7500 \mathrm{~L} / \mathrm{L}_{\text {cat }} / \mathrm{hr}$, and $300,315,324$, and $337^{\circ} \mathrm{C}$ at $11,000 \mathrm{~L} / \mathrm{L}_{\text {cat }} / \mathrm{hr}$. Temperature control was very good during this test at all conditions, with the catalyst temperature varying $\pm 2{ }^{\circ} \mathrm{C}$ about the reported temperature for each test condition.

This catalyst was not very reactive as indicated by the low carbon conversions shown in Figure B.29. There was also very little effect of the space velocity on the carbon conversions probably because they were so low. The $\mathrm{C}_{2}+$-oxygenate STYs were also low, as expected, reaching a maximum value of $150 \mathrm{~g} / \mathrm{L}_{\text {cat }} / \mathrm{hr}$ at $315^{\circ} \mathrm{C}$ as shown in Figure B.30. Higher temperatures produced significantly lower STYs, suggesting a possible change in the catalyst performance at higher temperatures. Carbon selectivity to $\mathrm{C}_{2}+$ oxygenates decreased with increasing temperature, as shown in Figure B.31, which is consistent with the other catalysts. The catalyst also showed an increasing carbon selectivity of $\mathrm{C}_{2}+$ oxygenates to $\mathrm{C}_{2}+$ alcohols with increasing temperature as shown in Figure B.32, reaching a maximum carbon selectivity ratio of 0.56 at $337^{\circ} \mathrm{C}$. The space velocity did not appear to have any significant effect on the carbon selectivity to $\mathrm{C}_{2}+$ oxygenates or the $\mathrm{C}_{2}+$ oxygenate: $\mathrm{C}_{2}+$ alcohol carbon selectivity ratio.

Figure B.33 shows the carbon selectivity to the various hydrocarbon fractions. The high carbon selectivity at $256^{\circ} \mathrm{C}$ is attributed to no liquid product being recovered at this condition. It appears that the selectivity to hydrocarbon liquids decreased to zero above $276^{\circ} \mathrm{C}$. The selectivity to $\mathrm{C}_{2}+$ hydrocarbon gases also decreased. 


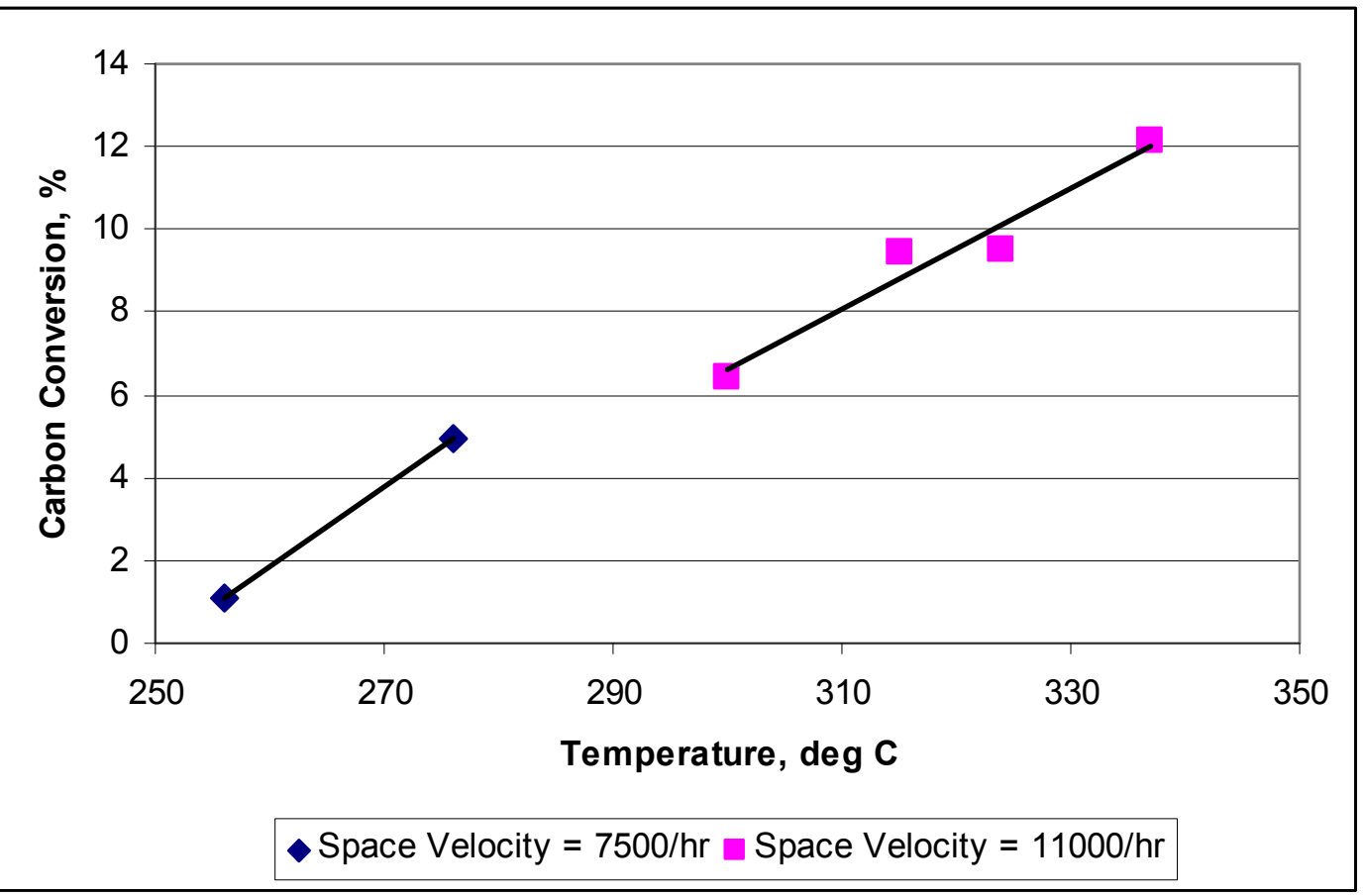

Figure B.29. Carbon Conversion for the Cu-Promoted Catalyst

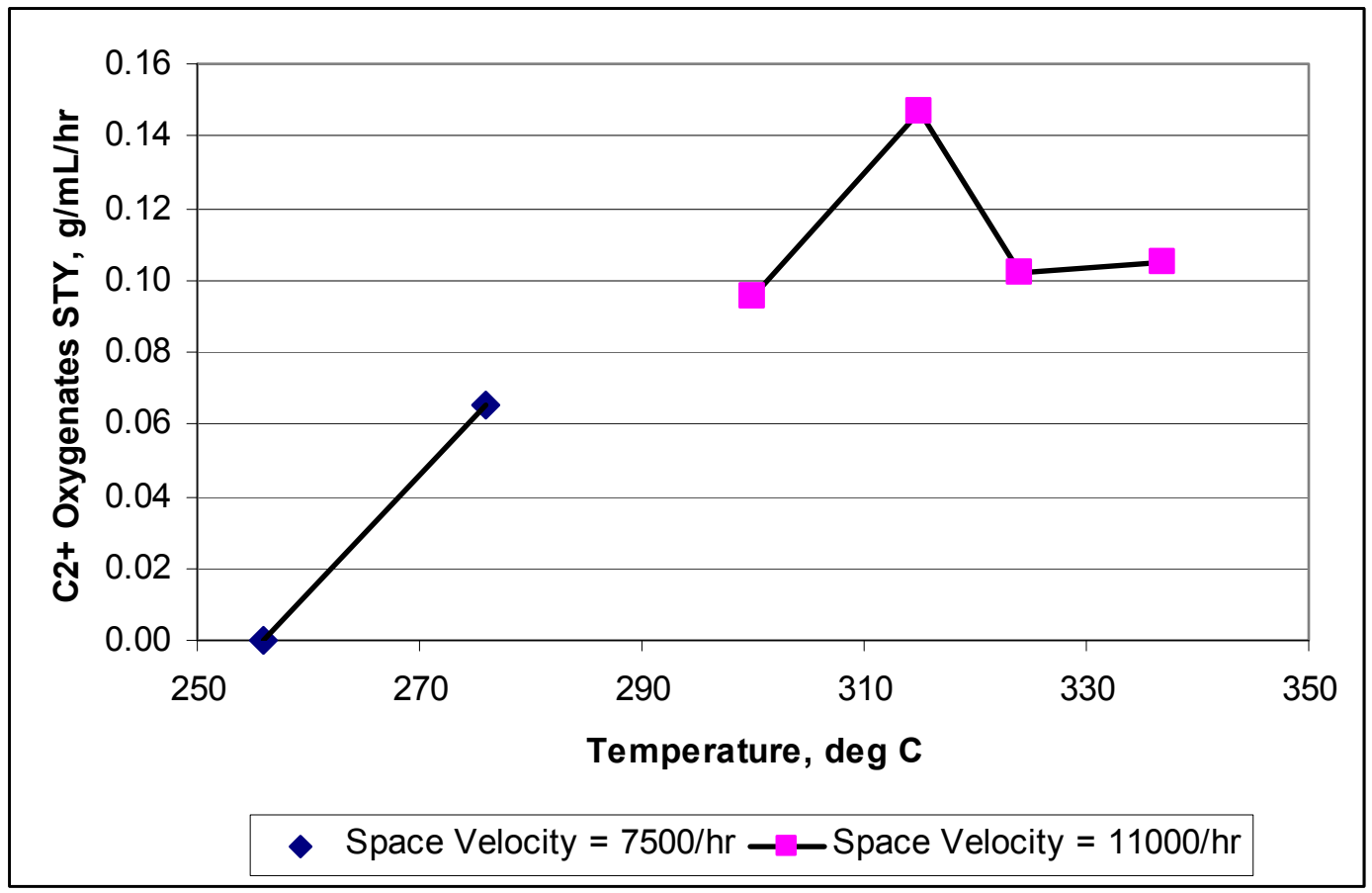

Figure B.30. $\mathrm{C}_{2}+-$ Oxygenate $\mathrm{STY}$ s for the $\mathrm{Cu}$-Promoted Catalyst 


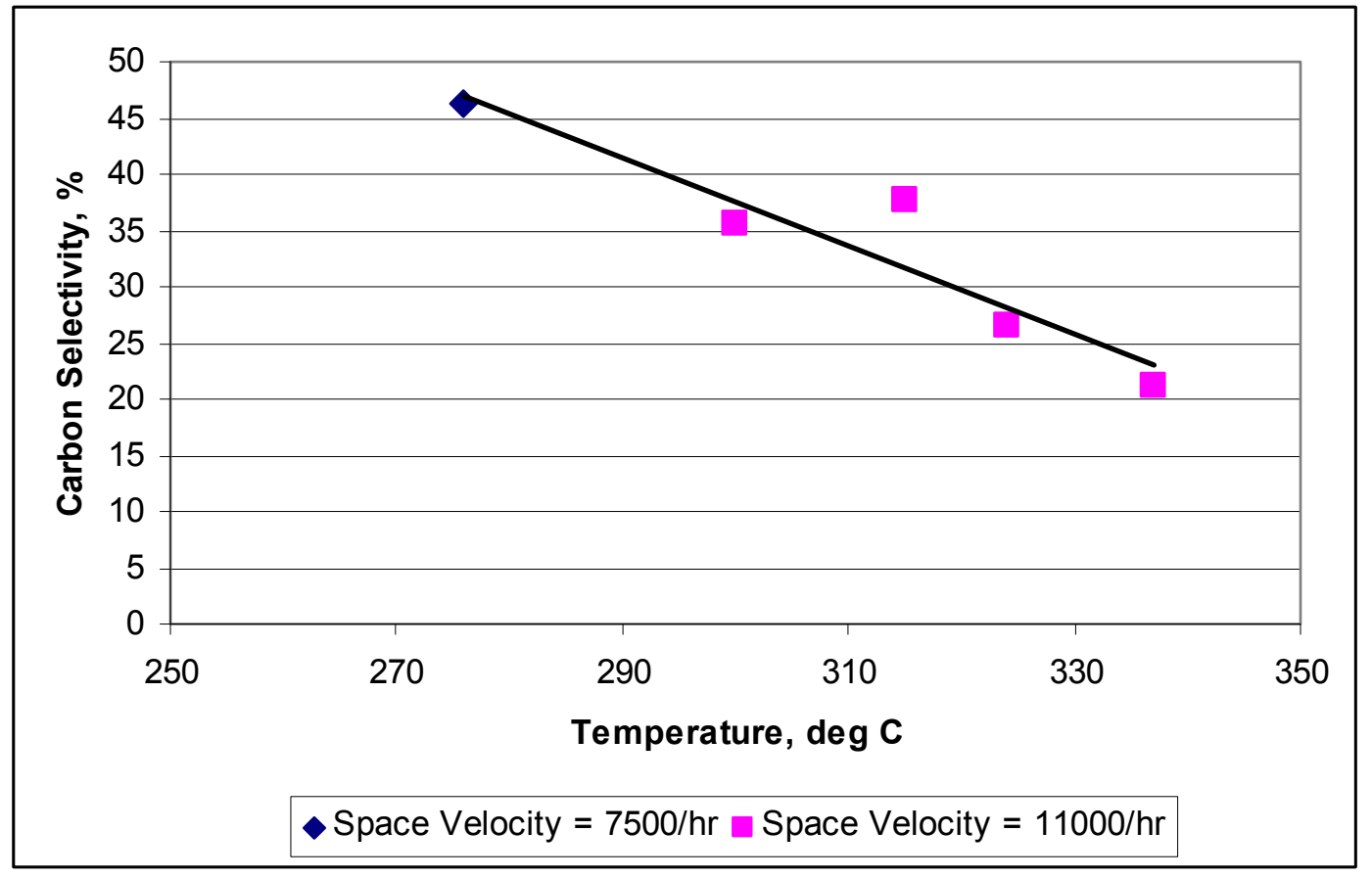

Figure B.31. Converted Carbon Selectivity to $\mathrm{C}_{2}+$ Oxygenates for the $\mathrm{Cu}$-Promoted Catalyst

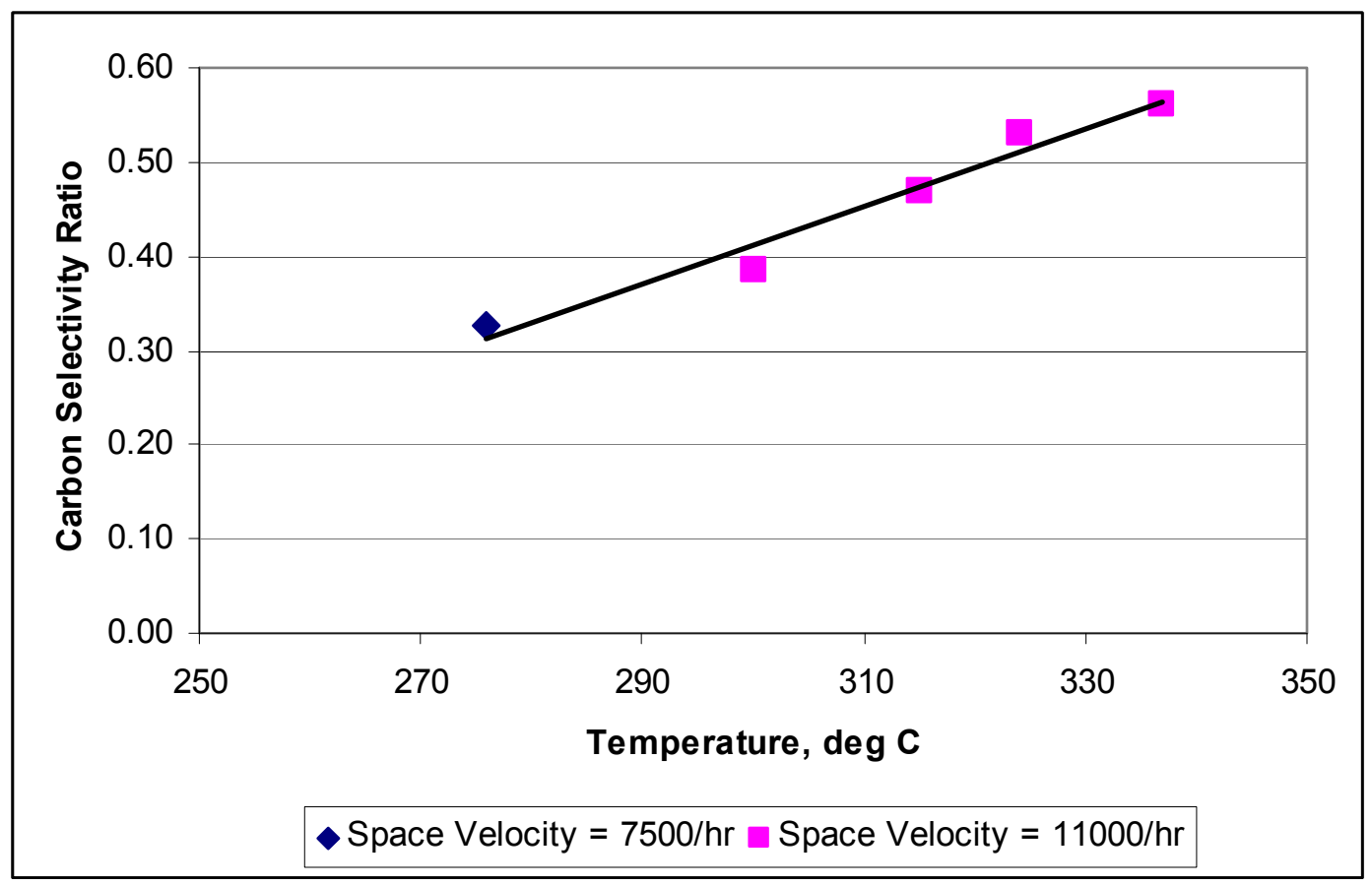

Figure B.32. Carbon Selectivity of All Oxygenates to $\mathrm{C}_{2}+$ Alcohols for the Cu-Promoted Catalyst 


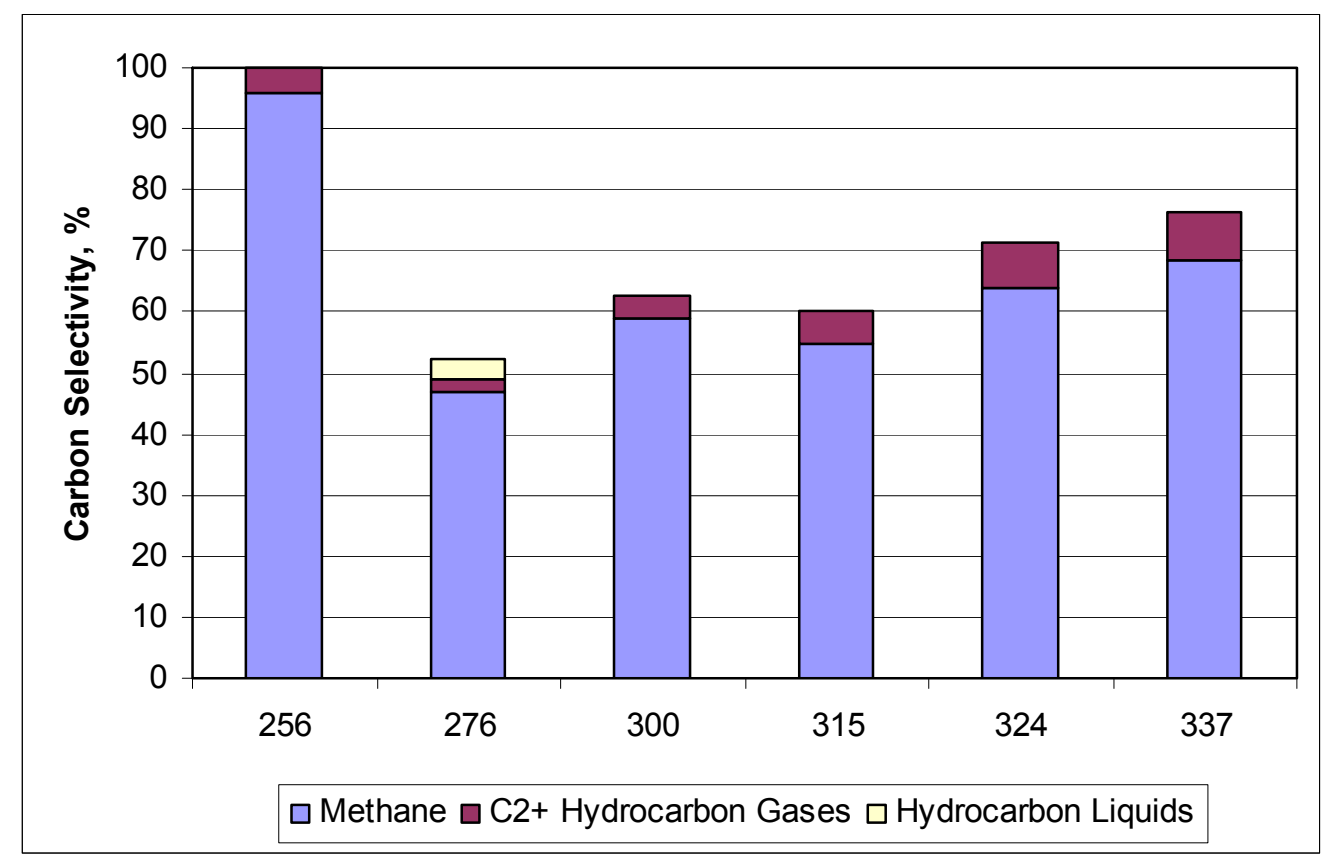

Figure B.33. Comparison of Hydrocarbon Selectivities for the Cu-Promoted Catalyst

\section{B.8 Co-Promoted Catalyst}

The Co-promoted catalyst $\left(\mathrm{RhMnCo} / \mathrm{SiO}_{2}\right)$ was reduced at a maximum temperature of $350^{\circ} \mathrm{C}$ and tested using the hot oil circulating system to heat the reactor. The testing sequence was 256, 256, and $276^{\circ} \mathrm{C}$ at $7500 \mathrm{~L} / \mathrm{L}_{\text {cat }} / \mathrm{hr}$, and 299,323 , and $307^{\circ} \mathrm{C}$ at $11,000 \mathrm{~L} / \mathrm{L}_{\text {cat }} / \mathrm{hr}$. Temperature control was very good during testing with the catalyst temperature varying $\pm 2{ }^{\circ} \mathrm{C}$ about the reported temperature for each test condition.

Carbon conversion increased with increasing temperature as shown in Figure B.34, but did not appear to be significantly affected by the space velocity over the range of conditions examined. Figure B.35 shows that the $\mathrm{C}_{2}+$-oxygenate STY peaked between 300 and $307^{\circ} \mathrm{C}$ at approximately $350 \mathrm{~g} / \mathrm{L}_{\text {cat }} / \mathrm{hr}$. Carbon selectivity to $\mathrm{C}_{2}+$ oxygenates decreased with increasing temperature as shown in Figure B.36, and also did not appear to be affected by the space velocity. Figure B.37 shows that the carbon selectivity of $\mathrm{C}_{2}+$ oxygenates to $\mathrm{C}_{2}+$ alcohols not only increased with increasing temperature, but also became more temperature sensitive with increasing temperature, unlike previously tested catalysts.

Examination of Figures B.34 through B.37 indicates that the catalyst did not show significant evidence of deactivation after testing at $323^{\circ} \mathrm{C}$, with respect to carbon conversion, $\mathrm{C}_{2}+$-oxygenate STY, carbon selectivity to $\mathrm{C}_{2}+$ oxygenates or the selectivity of the oxygenates to the alcohols. However, Figure B.38 suggests that the catalyst was still undergoing changes because of the significant decrease in carbon selectivity to hydrocarbon liquids best illustrated by comparing the hydrocarbon selectivity at $299^{\circ} \mathrm{C}$ (the third condition tested) and $307^{\circ} \mathrm{C}$ (the last condition tested). Both conditions had similar carbon selectivity to all hydrocarbons, but the $307^{\circ} \mathrm{C}$ condition had almost no hydrocarbon liquids while the hydrocarbon liquids accounted for more than $10 \%$ of the carbon selectivity to all hydrocarbons for the $299^{\circ} \mathrm{C}$ condition. 


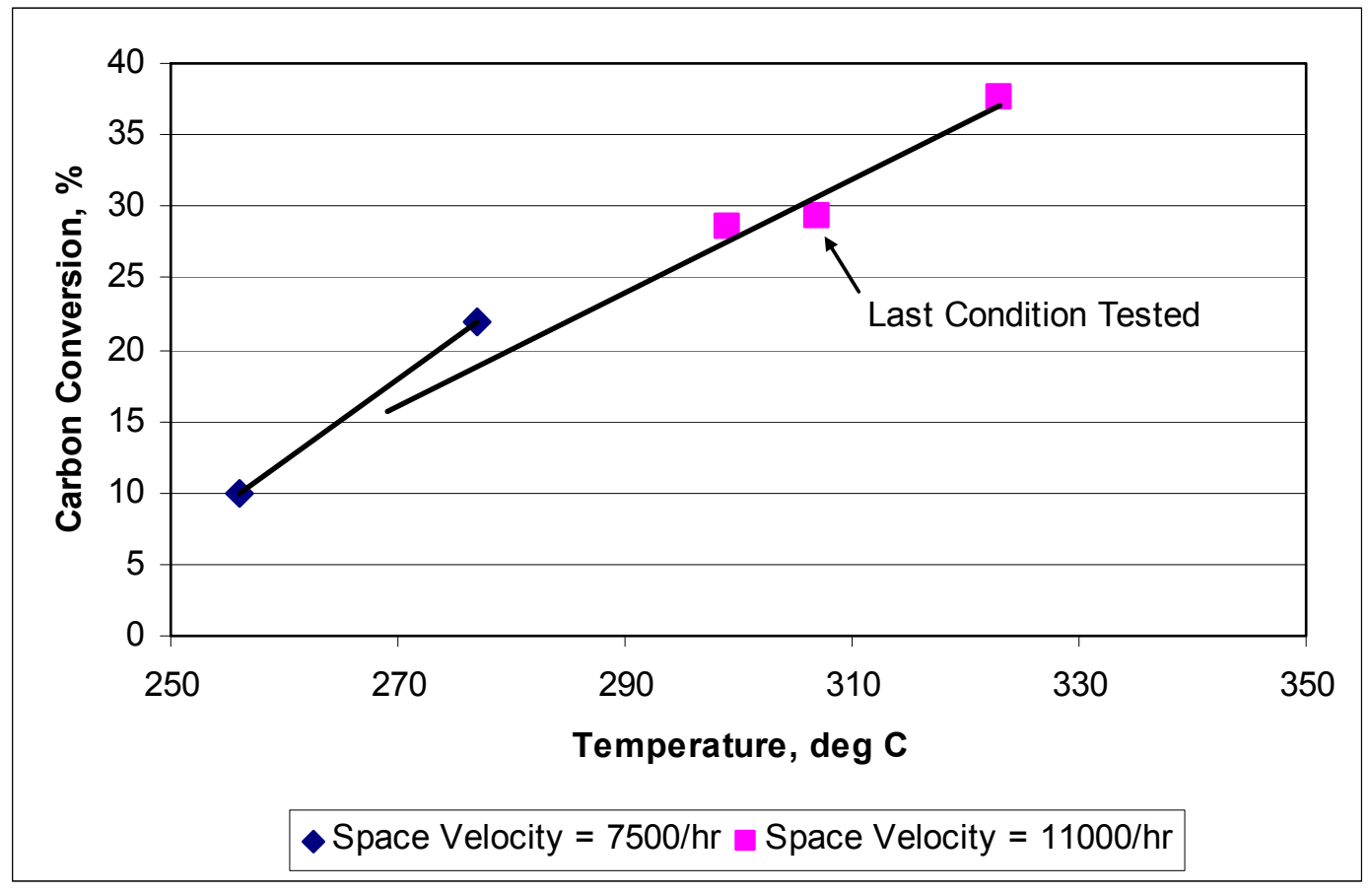

Figure B.34. Carbon Conversion for the Co-Promoted Catalyst

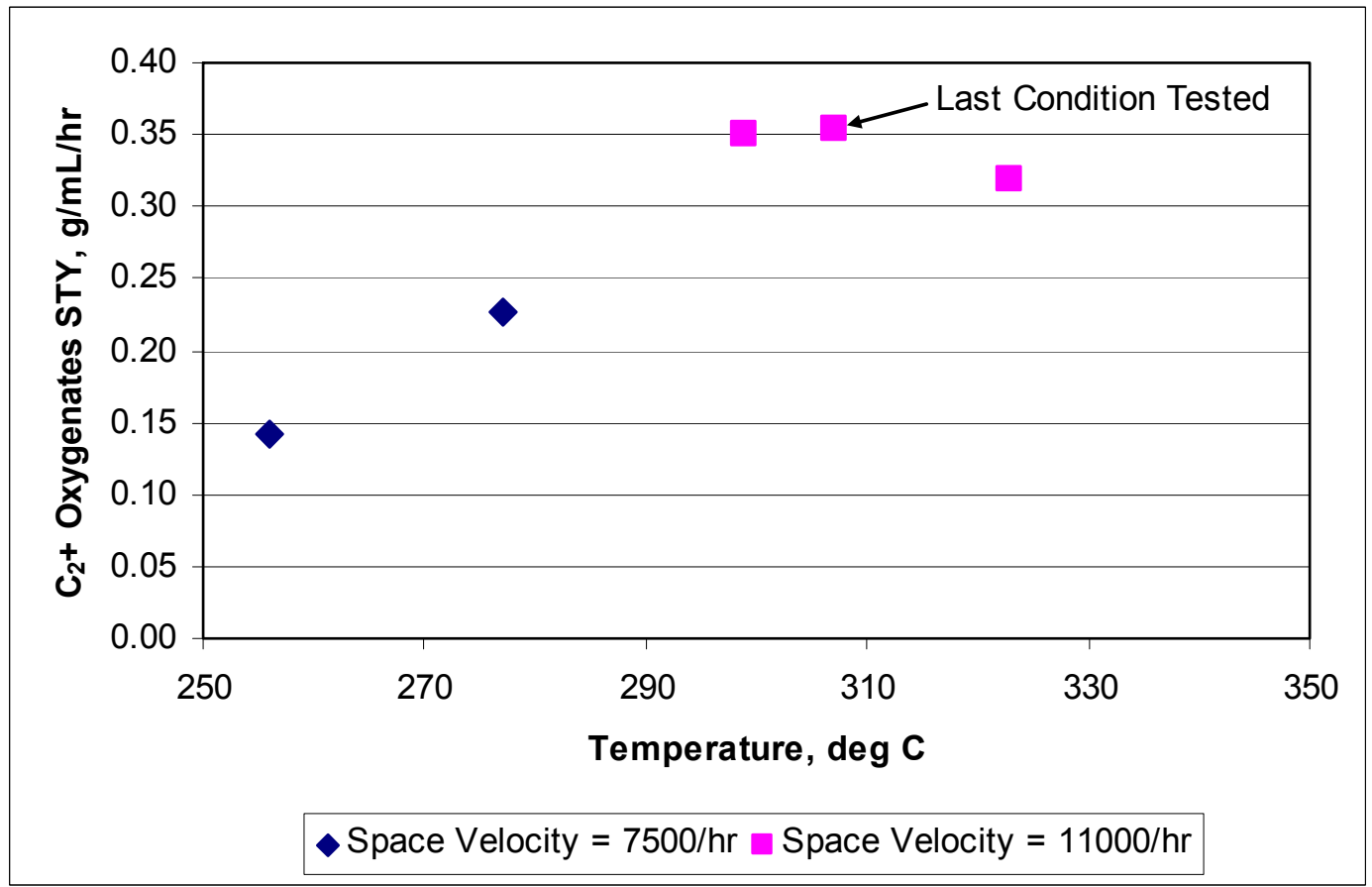

Figure B.35. $\mathrm{C}_{2}+-$ Oxygenate STYs for the Co-Promoted Catalyst 


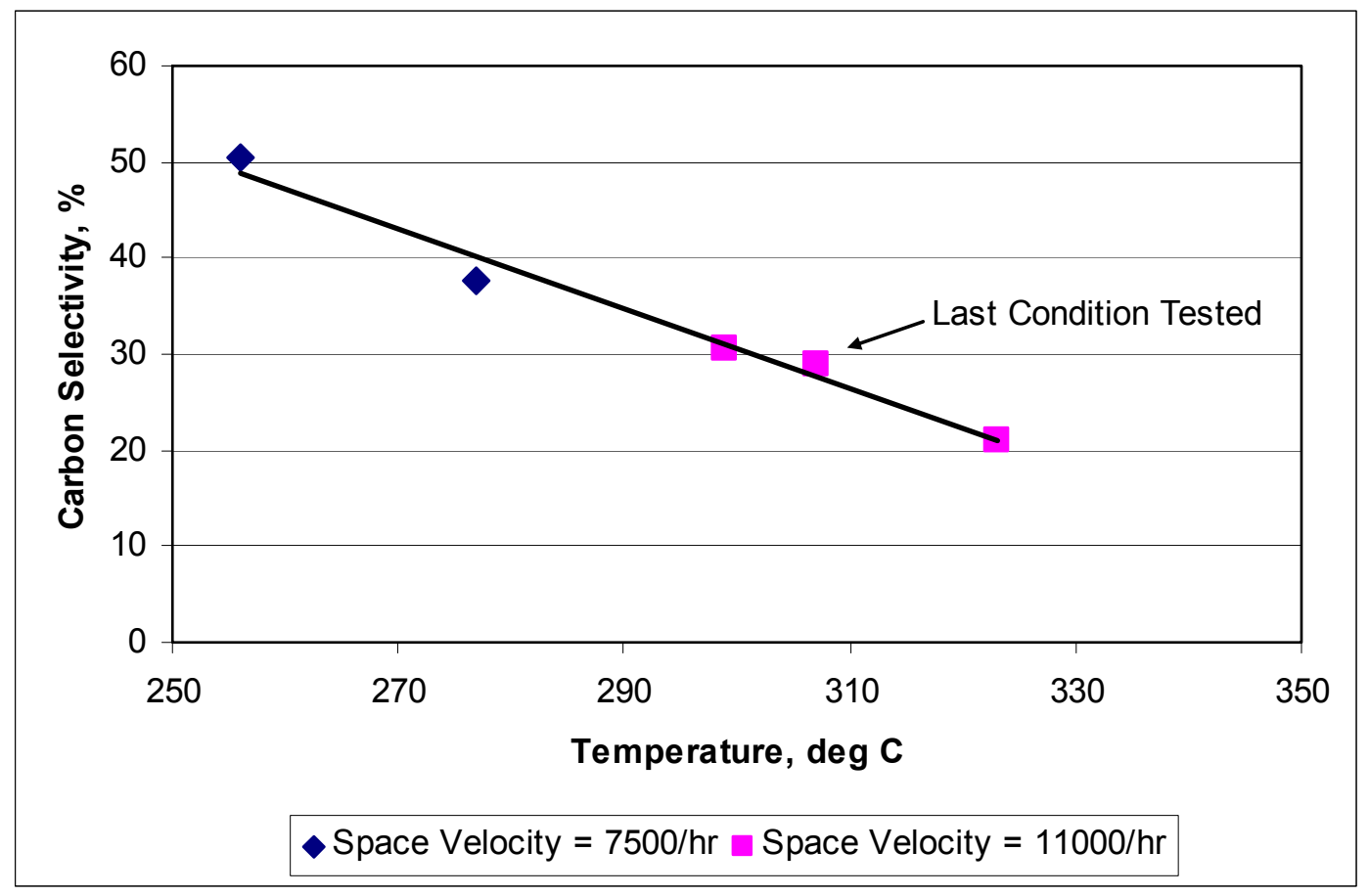

Figure B.36. Converted Carbon Selectivity to $\mathrm{C}_{2}+$ Oxygenates for the Co-Promoted Catalyst

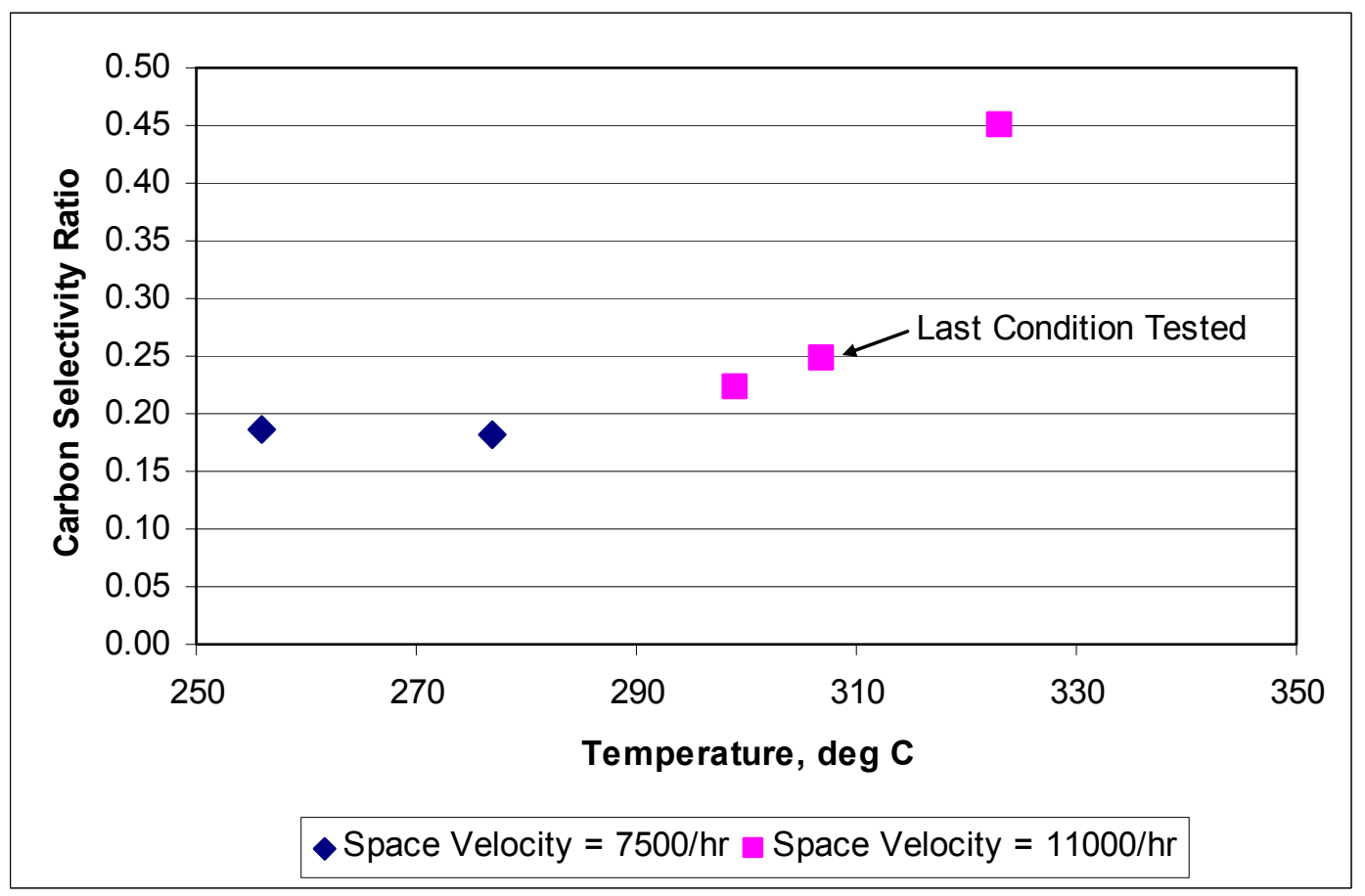

Figure B.37. Carbon Selectivity of All Oxygenates to $\mathrm{C}_{2}+$ Alcohols for the Co-Promoted Catalyst 


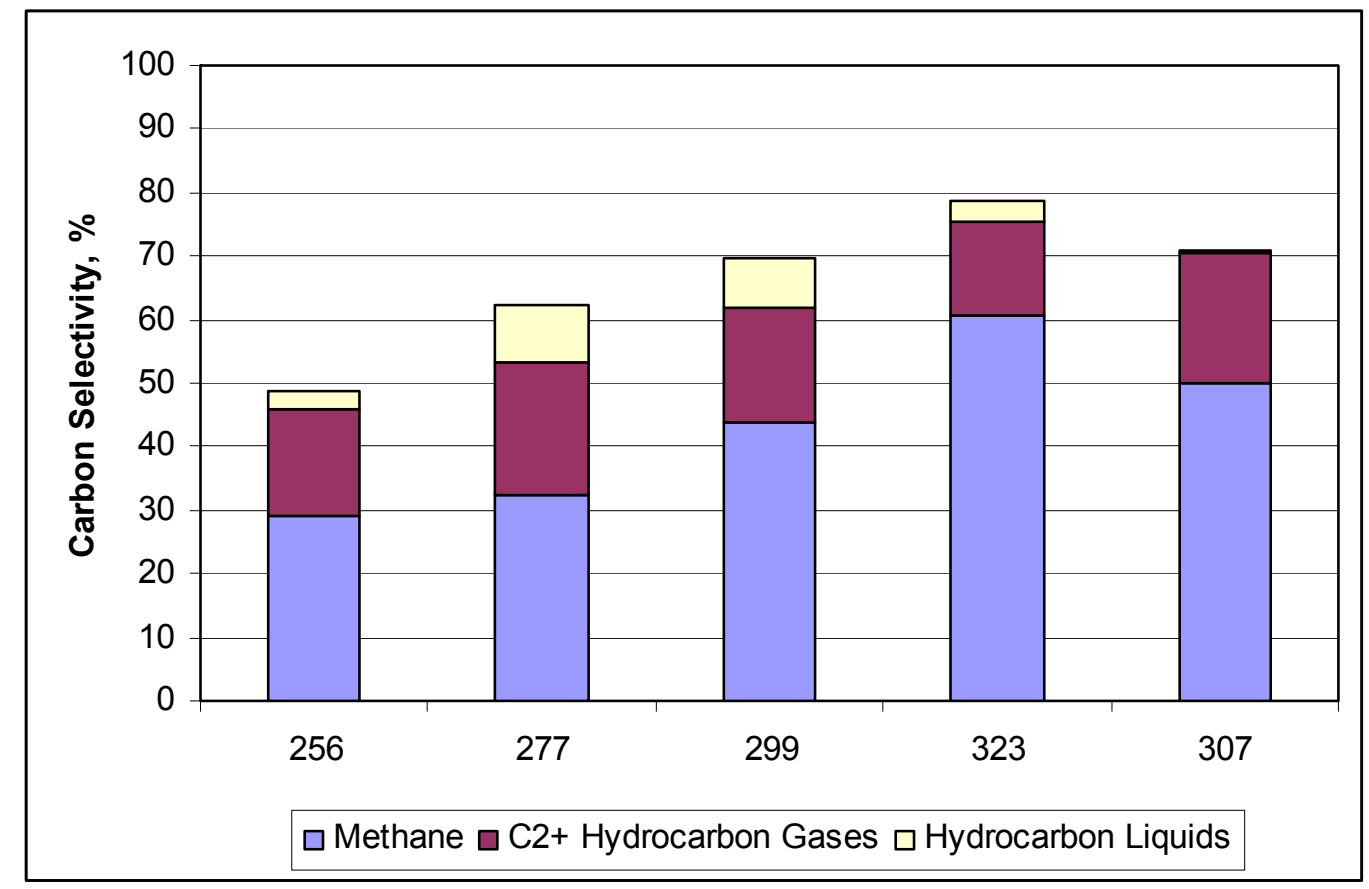

Figure B.38. Comparison of Hydrocarbon Selectivity for the Co-Promoted Catalyst

\section{B.9 Mo-Promoted Catalyst}

The Mo-promoted catalyst $\left(\mathrm{RhMnMo} / \mathrm{SiO}_{2}\right)$ was reduced at the maximum temperature of $350^{\circ} \mathrm{C}$ and tested using the hot oil circulating system to heat the reactor. The testing sequence was 256 and $275^{\circ} \mathrm{C}$ at $7500 \mathrm{~L} / \mathrm{L}_{\text {cat }} / \mathrm{hr} ; 298,314$, and $324^{\circ} \mathrm{C}$ at $11,000 \mathrm{~L} / \mathrm{L}_{\text {cat }} / \mathrm{hr}$; and 324 and $316^{\circ} \mathrm{C}$ at $15,000 \mathrm{~L} / \mathrm{L}_{\text {cat }} / \mathrm{hr}$.

This catalyst behaved very differently from previously tested catalysts. There were no problems in sustaining catalyst temperature for the test conditions at 256 and $275^{\circ} \mathrm{C}$; however, when the temperature of the circulating oil was increased to elevate the catalyst temperature to approximately $300^{\circ} \mathrm{C}$, the temperature rapidly oscillated between approximately 280 and $330^{\circ} \mathrm{C}$ for about 7 hours before abruptly dropping to $275^{\circ} \mathrm{C}$ and then slowly rising to approximately $290^{\circ} \mathrm{C}$ over a 3-hour period. Sporadic clusters of temperature oscillations continued for more than 24 hours. Liquid sample and data collection took place after the catalyst stabilized at $298^{\circ} \mathrm{C}$. When the oil temperature was increased to establish steady conditions at approximately $314^{\circ} \mathrm{C}$, sporadic periods of temperature oscillations between 315 and $330^{\circ} \mathrm{C}$ initially occurred, but these oscillations eventually subsided before liquid sample and data collection took place at this condition. Subsequently, no problems with the test conditions were encountered were experienced, although a slow deactivation of the catalyst was indicated by gradual temperature decreases of a few degrees during sample collection.

Figures B.39 through B.43 show catalyst behaviors very different from that observed with previously tested catalysts. Carbon conversion (Figure B.39) appears to be very sensitive to the space velocity, which is unlike most of the other catalysts. Furthermore, the shallowness of the slope of the curve for carbon conversion at $11,000 \mathrm{~L} / \mathrm{L}_{\text {cat }} / \mathrm{hr}$ space velocity relative to that for carbon conversion at $15,000 \mathrm{~L} / \mathrm{L}_{\text {cat }} / \mathrm{hr}$ space velocity suggests that deactivation of the catalyst at progressively higher 
temperatures $\left(11,000 \mathrm{~L} / \mathrm{L}_{\text {cat }} / \mathrm{hr}\right.$ space velocity) was off-setting the expected response to increasing temperature, while progressively lower temperatures (at $15,000 \mathrm{~L} / \mathrm{L}_{\mathrm{cat}} / \mathrm{hr}$ space velocity) were augmenting the expected response to decreasing temperature. Similar indications of deactivation are present in Figure B.40 for the $\mathrm{C}_{2}+$-oxygenate STYs for conditions at $15,000 \mathrm{~L} / \mathrm{L}_{\text {cat }} / \mathrm{hr}$ space velocity, where the STY at $316^{\circ} \mathrm{C}$ (the last condition tested) was lower than the STY at $324^{\circ} \mathrm{C}$, as well as lower than the STY at $314^{\circ} \mathrm{C}$ and $11,000 \mathrm{~L} / \mathrm{L}_{\text {cat }} / \mathrm{hr}$ space velocity. Figure B.41, which shows temperature and space velocity effects on carbon selectivity to $\mathrm{C}_{2}+$ oxygenates, suggests a very significant apparent effect of space velocity on carbon selectivity, at least between 7500 and $11,000 \mathrm{~L} / \mathrm{L}_{\text {cat }} / \mathrm{hr}$ space velocities. The selectivity of all oxygenates to $\mathrm{C}_{2}+$ alcohols also was irregular, as shown in Figure B.42, with the selectivity occurring at approximately $325^{\circ} \mathrm{C}$ and a $11,000 \mathrm{~L} / \mathrm{L}_{\text {cat }} / \mathrm{hr}$ space velocity lower than at lower temperatures for the same space velocity and at higher space velocities at the same temperatures. This is opposite to the expected behavior of higher temperatures and lower space velocities favoring hydrogenation of non-alcoholic oxygenates to alcohols. Figure B.43 showed a decrease in the production of hydrocarbon liquids at $300^{\circ} \mathrm{C}$ or higher, suggesting deactivation of the catalyst towards chain growth.

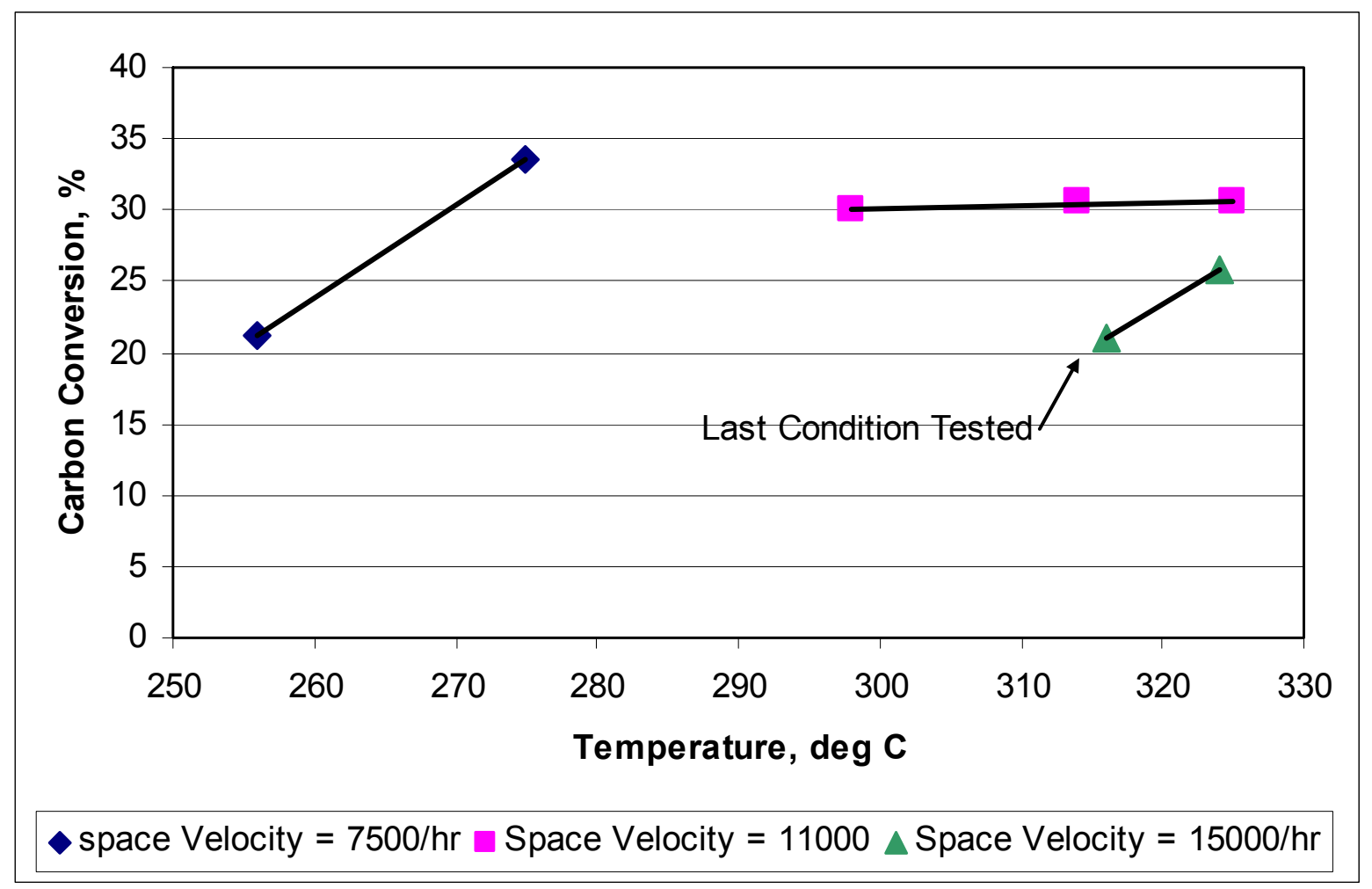

Figure B.39. Carbon Conversion for the Mo-Promoted Catalyst. 


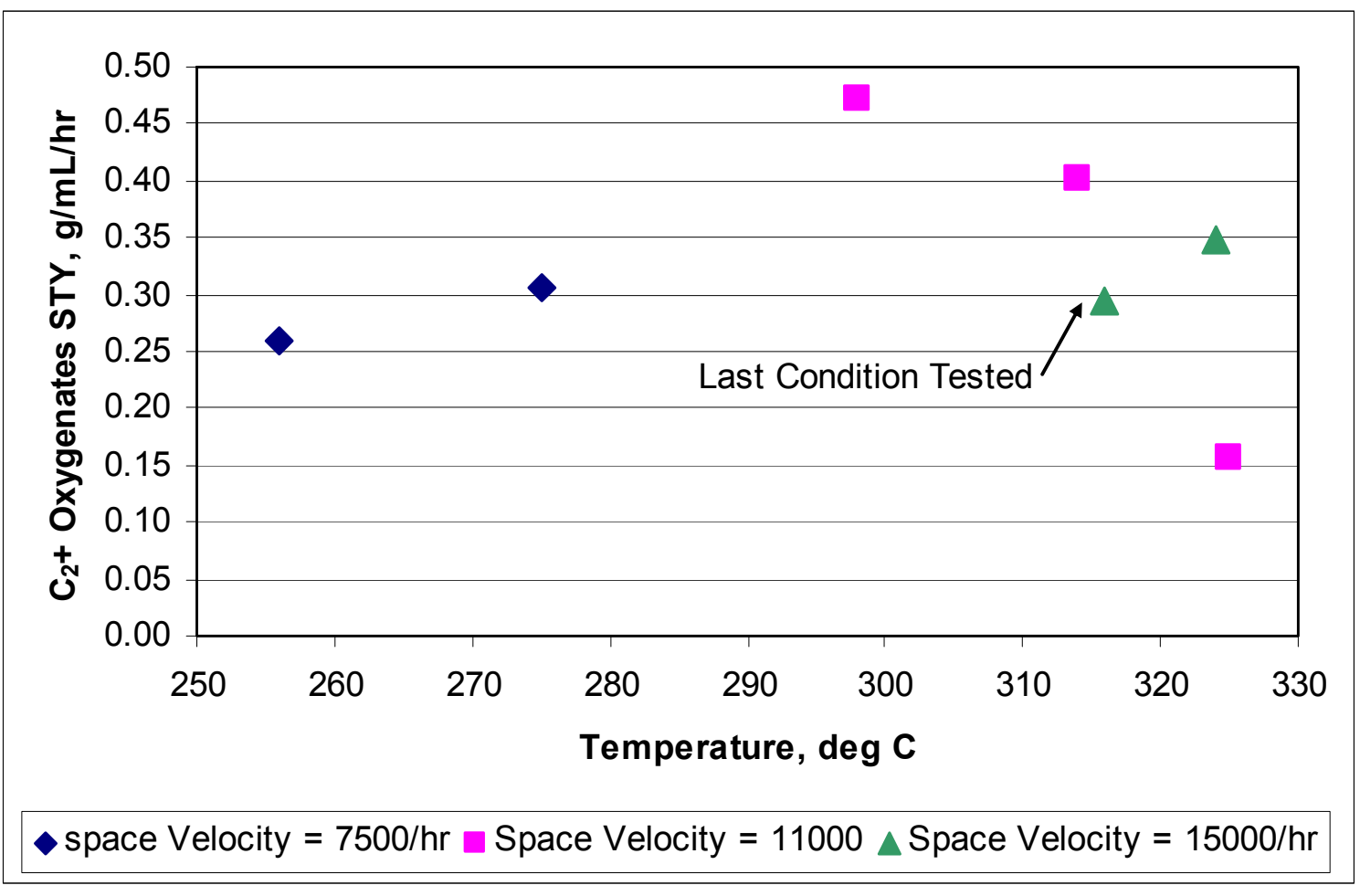

Figure B.40. $\mathrm{C}_{2}+$-Oxygenate STYs for the Mo-Promoted Catalyst

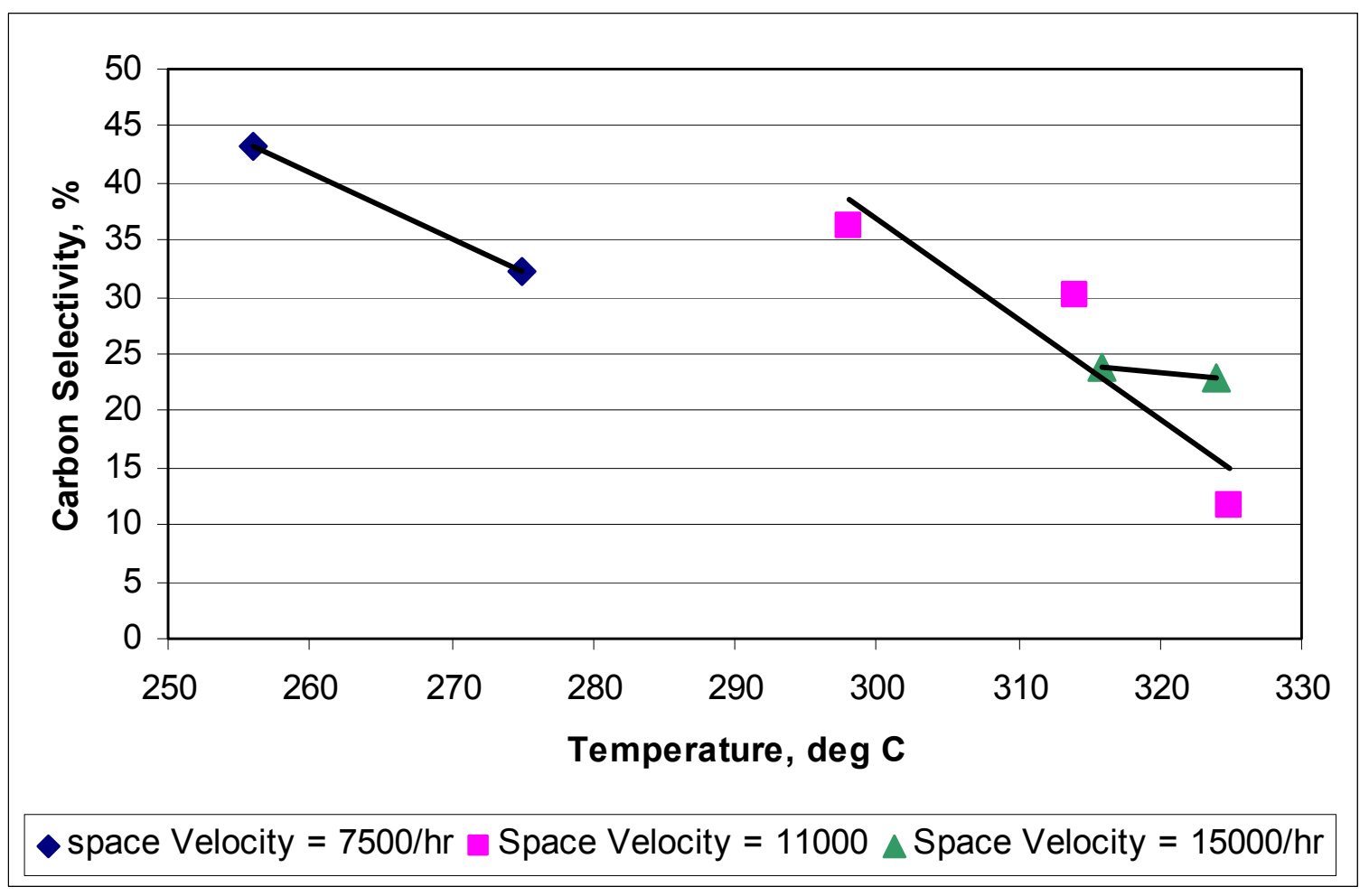

Figure B.41. Converted Carbon Selectivity to $\mathrm{C}_{2}+$ Oxygenates for the Mo-Promoted Catalyst 


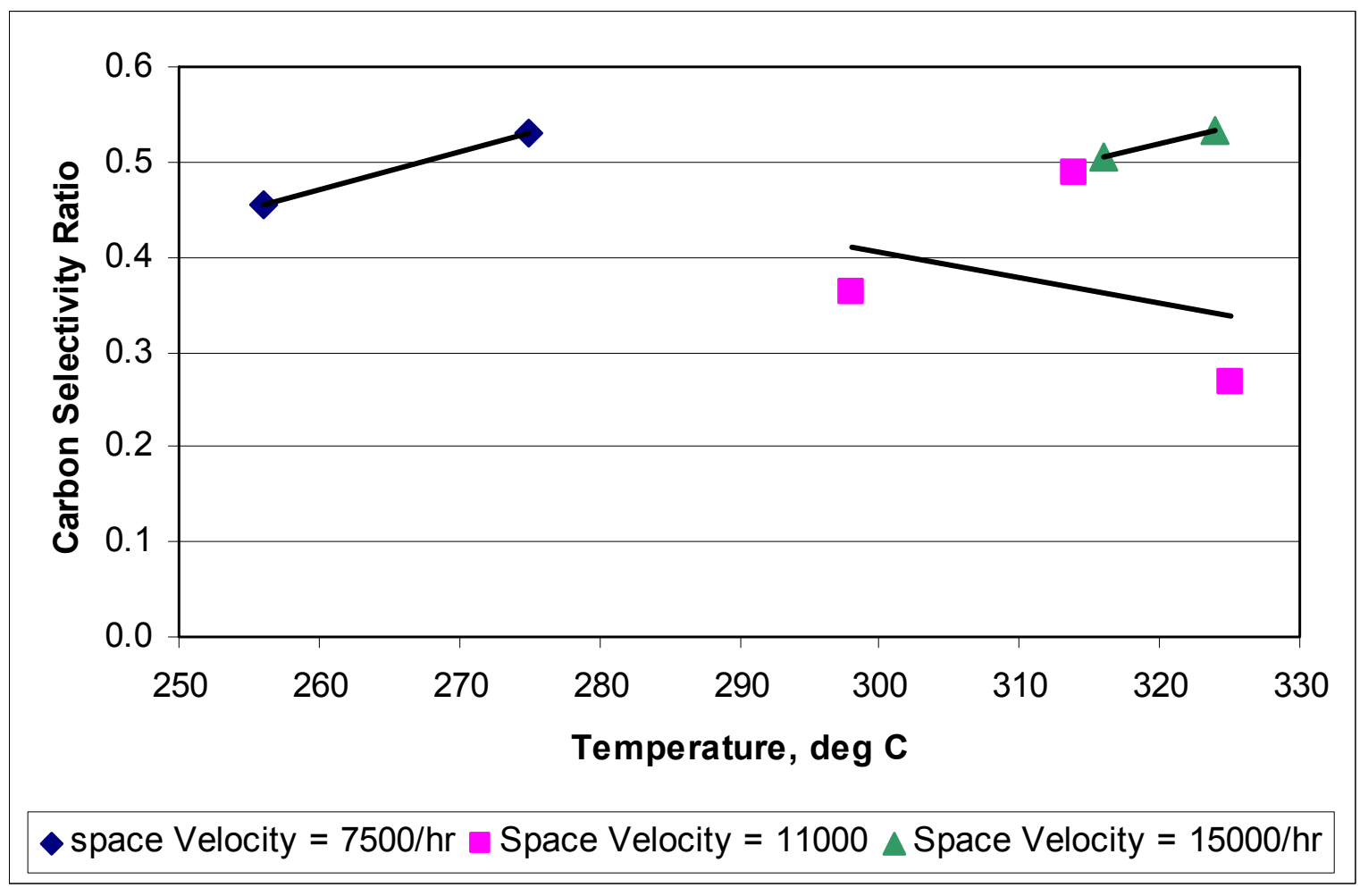

Figure B.42. Carbon Selectivity of All Oxygenates to $\mathrm{C}_{2}+$ Alcohols for the Mo-Promoted Catalyst

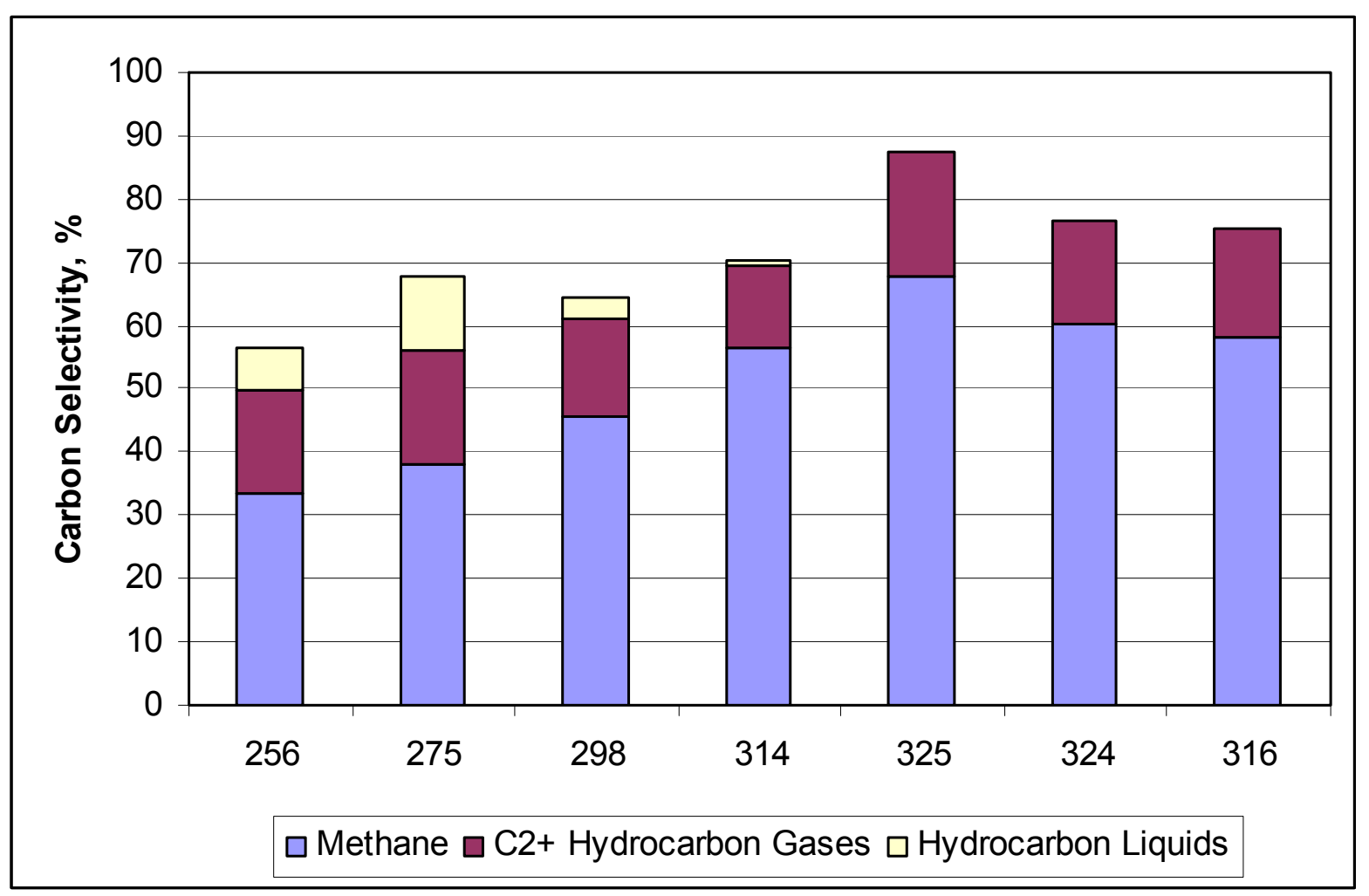

Figure B.43. Comparison of Hydrocarbon Selectivity for the Mo-Promoted Catalyst 


\section{B.10 Pd-Promoted Catalyst}

The Pd-promoted catalyst $\left(\mathrm{RhMnPd} / \mathrm{SiO}_{2}\right)$ catalyst was reduced at the maximum temperature of $350^{\circ} \mathrm{C}$ and tested using the hot oil circulating system to heat the reactor. The catalyst testing sequence was 256 and $276^{\circ} \mathrm{C}$ at $7500 \mathrm{~L} / \mathrm{L}_{\text {cat }} / \mathrm{hr}$, and $300,323,314$, and $302^{\circ} \mathrm{C}$ at $11,000 \mathrm{~L} / \mathrm{L}_{\text {cat }} / \mathrm{hr}$. The temperature control was fairly good with the catalyst, varying $\pm 3^{\circ} \mathrm{C}$ degrees for each test condition.

This catalyst was only moderately reactive as indicated by the carbon conversions of between 10 and $22 \%$ for temperatures at or above approximately $300^{\circ} \mathrm{C}$ and an $11,000 \mathrm{~L} / \mathrm{L}_{\mathrm{cat}} / \mathrm{hr}$ space velocity, as shown in Figure B.44. There also appeared to be a decrease in activity when the temperature was raised to $323^{\circ} \mathrm{C}$, which may have stabilized, at subsequent testing conditions. This behavior also is reflected in the decrease in the $\mathrm{C}_{2}+$-oxygenate STYs after the catalyst temperature was raised to $323^{\circ} \mathrm{C}$ as shown in Figure B.45. Carbon selectivity to $\mathrm{C}_{2}+$ oxygenates followed a general trend of lower selectivity with higher reaction temperatures as shown in Figure B.47. The repeat condition at $302^{\circ} \mathrm{C}$ also indicates that selectivity to oxygenates was reduced after the catalyst was tested at $323^{\circ} \mathrm{C}$. Figure B. 47 indicates that the selectivity of all oxygenates to alcohols followed a regular trend of increasing selectivity with reaction temperature. The effect of an apparent deactivation of the catalyst upon reaching $323^{\circ} \mathrm{C}$ produced a small decrease the selectivity of oxygenates to alcohols as evidenced by the selectivity ratio of the repeat condition at $302^{\circ} \mathrm{C}$. According to Figure B.48, liquid hydrocarbons were only produced at $275^{\circ} \mathrm{C}$ and a $7500 \mathrm{~L} / \mathrm{L}_{\text {cat }} / \mathrm{hr}$ space velocity. Increasing the temperature to $300^{\circ} \mathrm{C}$ and the space velocity to $11,000 \mathrm{~L} / \mathrm{L}_{\text {cat }} / \mathrm{hr}$ did not significantly increase selectivity to hydrocarbons, and hydrocarbon liquids did not form. This could have resulted from the higher space velocity, but when significantly higher selectivity to hydrocarbons occurred when the temperature was further raised to $223^{\circ} \mathrm{C}$, no liquid hydrocarbons were formed and the selectivity to higher hydrocarbon gases remained unchanged. The higher selectivity to hydrocarbons in general and to higher hydrocarbon gases, as the temperature was subsequently dropped, suggests that the catalyst was still changing resulting in a shift from producing oxygenates to hydrocarbons over time and that the catalyst in fact may not have stabilized as was previously hypothesized. 


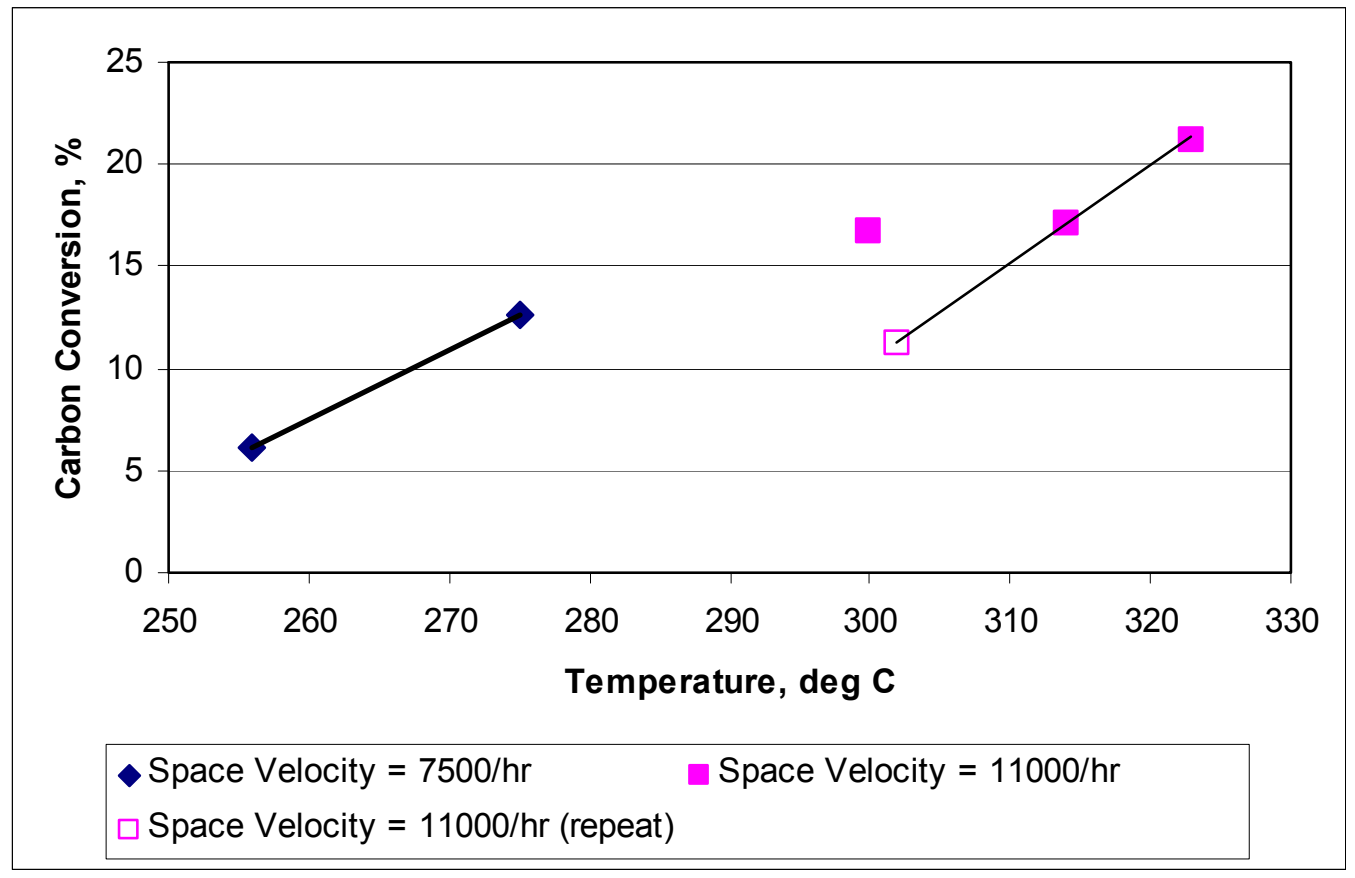

Figure B.44. Carbon Conversion for the Pd-Promoted Catalyst

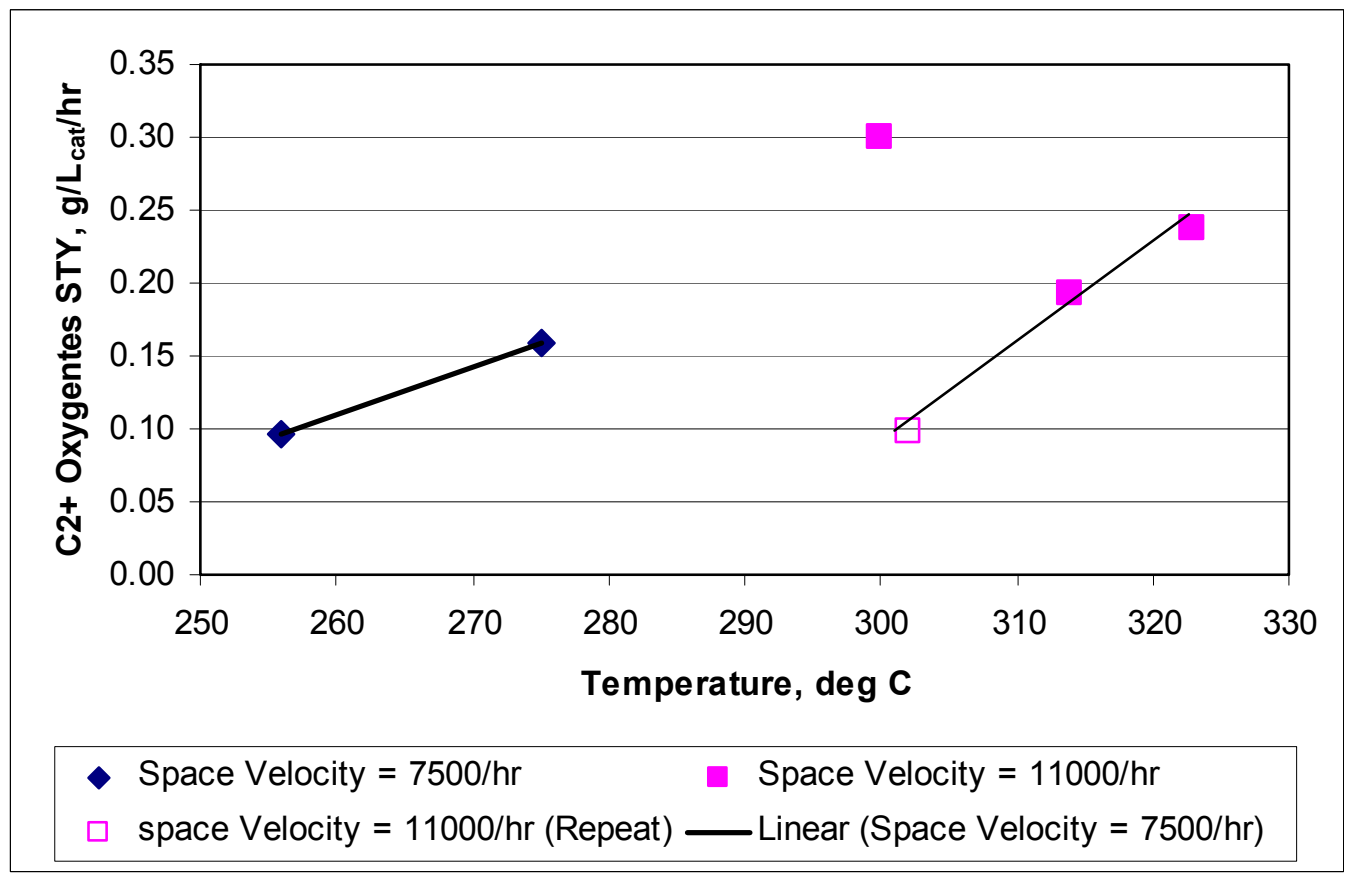

Figure B.45. $\mathrm{C}_{2}+-$ Oxygenate STYs for the Pd-Promoted Catalyst 


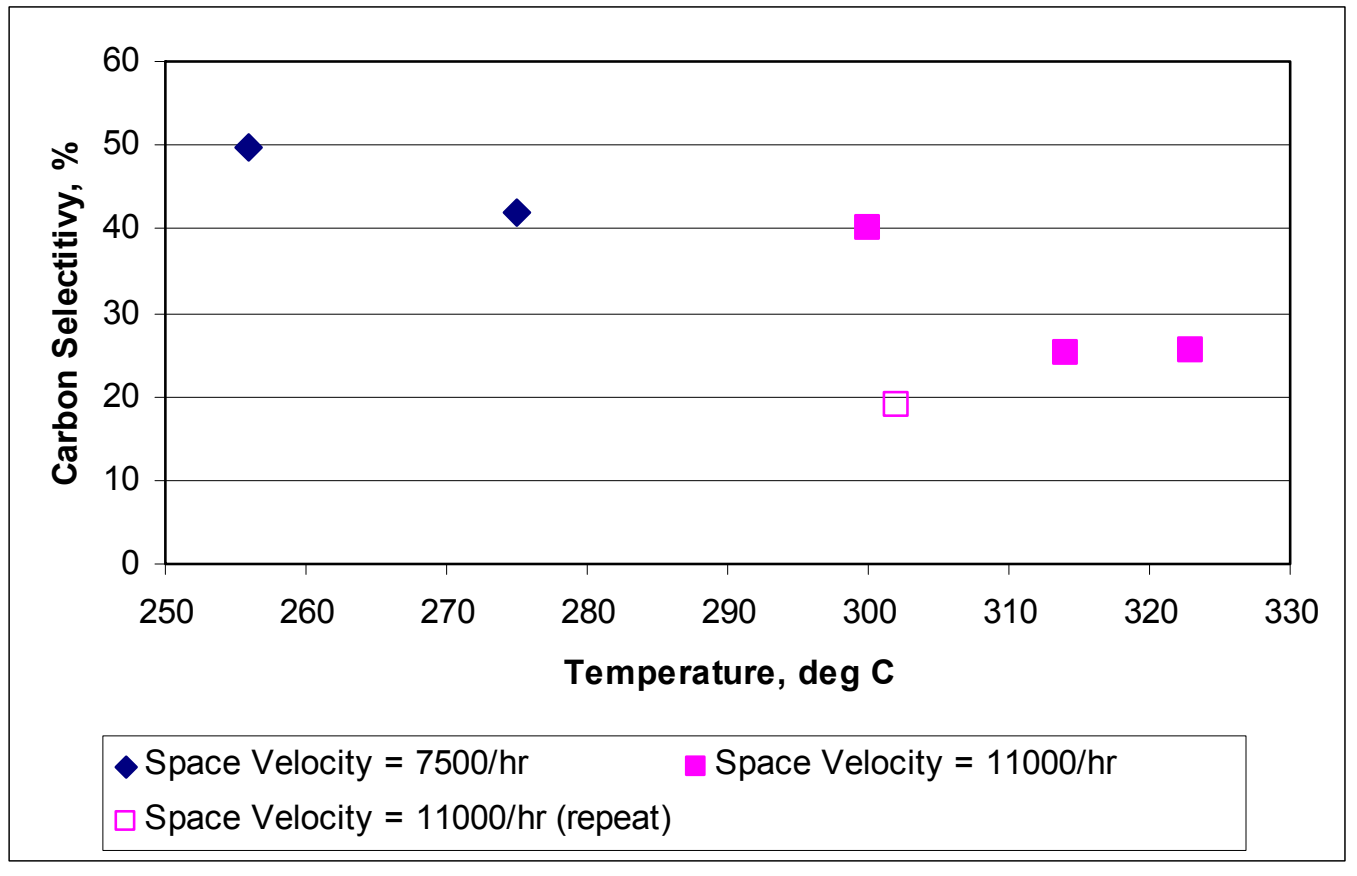

Figure B.46. Converted Carbon Selectivity to $\mathrm{C}_{2}+$ Oxygenates for the Pd-Promoted Catalyst

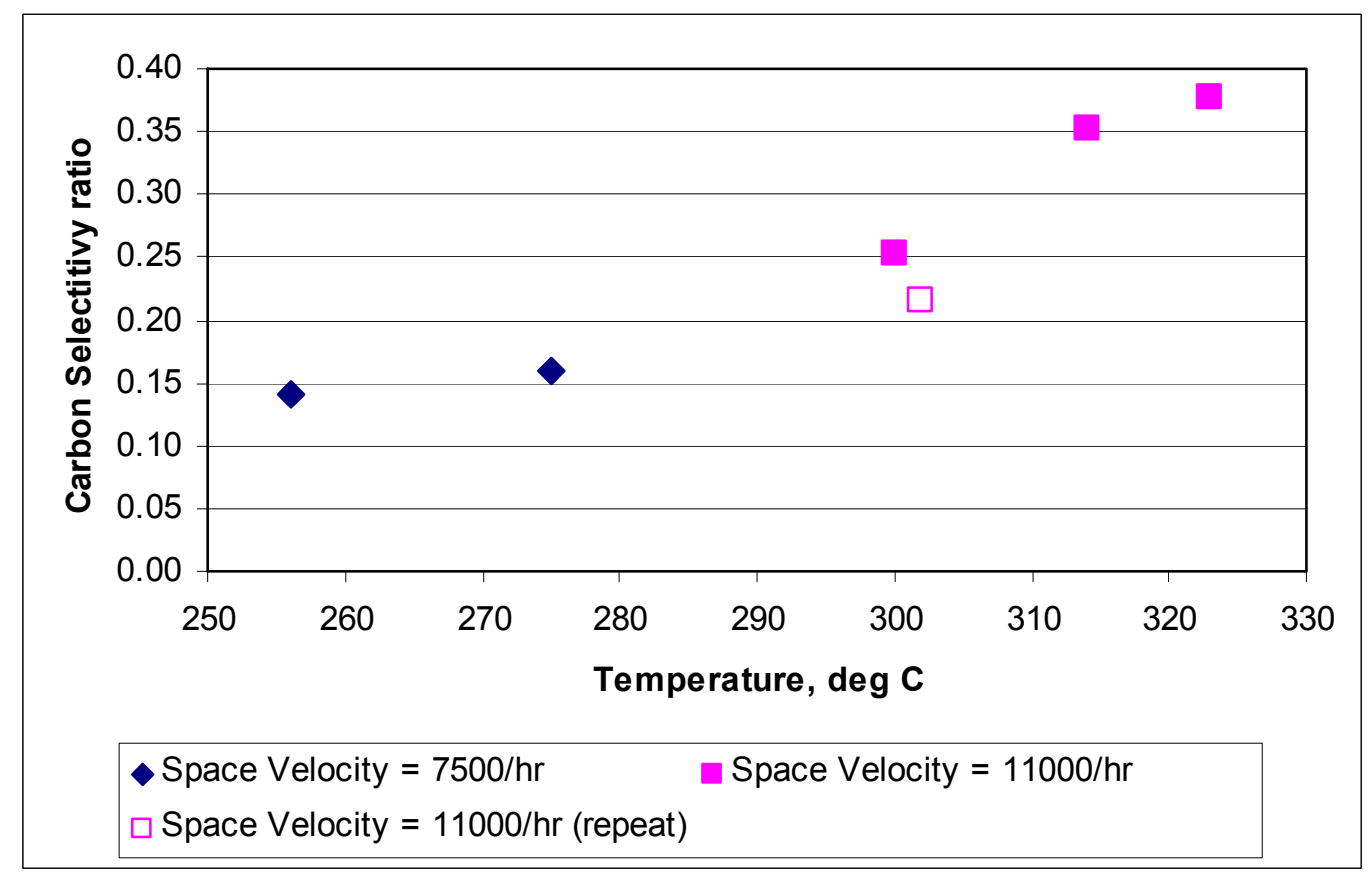

Figure B.47. Carbon Selectivity of All Oxygenates to $\mathrm{C}_{2}+$ Alcohols for the Pd-Promoted Catalyst 


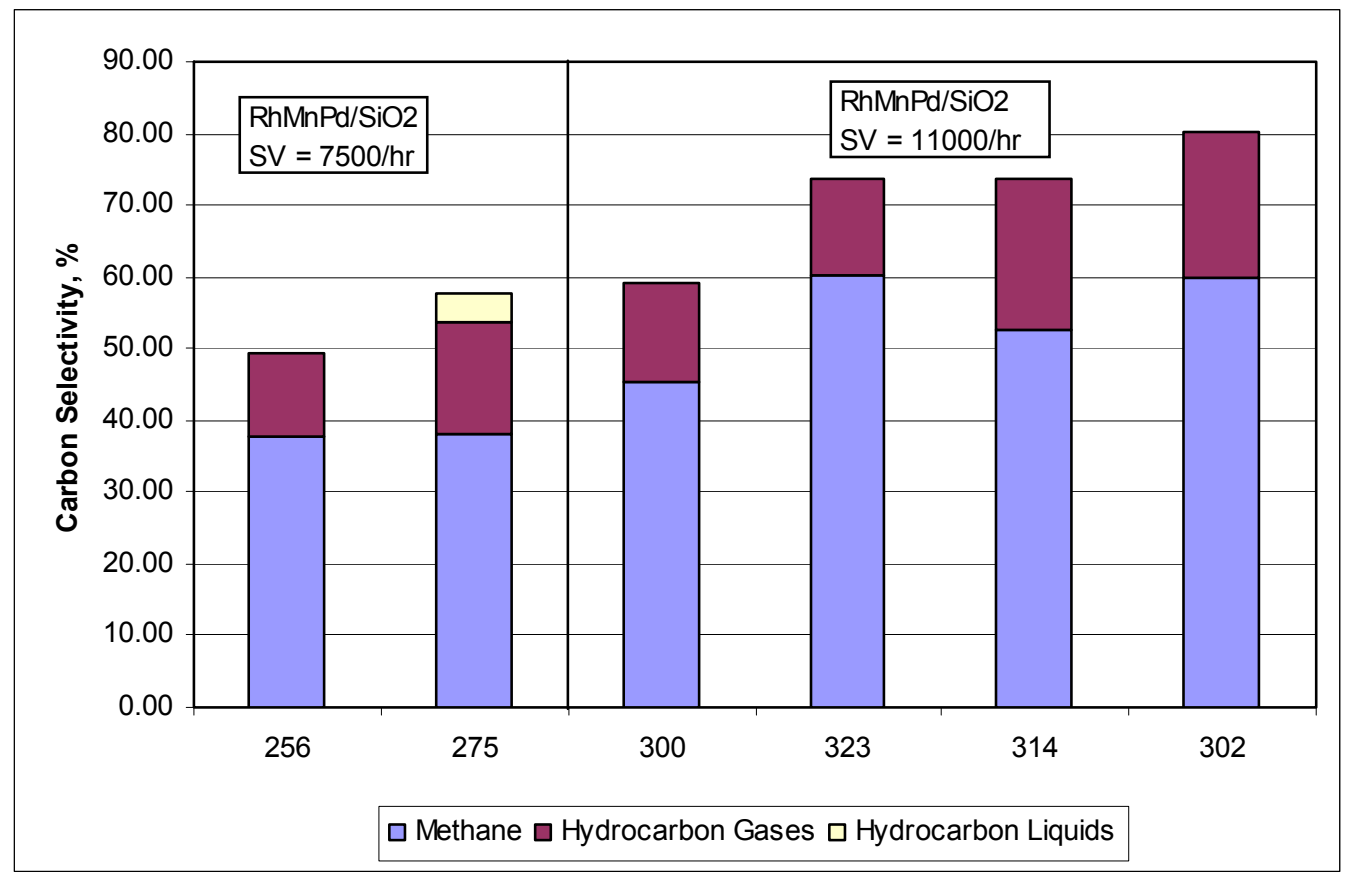

Figure B.48. Comparison of Hydrocarbon Selectivities for the Pd-Promoted Catalyst

\section{B.11 Platinum Promoted Catalyst}

The Pt-promoted catalyst $\left(\mathrm{RhMnPt} / \mathrm{SiO}_{2}\right)$ catalyst was reduced at the maximum temperature of $350^{\circ} \mathrm{C}$ and tested using the hot oil circulating system to heat the reactor. The testing sequence was 255 and $275^{\circ} \mathrm{C}$ at $7500 \mathrm{~L} / \mathrm{L}_{\text {cat }} / \mathrm{hr}$, and $306,300,313$, and $307^{\circ} \mathrm{C}$ at $11,000 \mathrm{~L} / \mathrm{L}_{\text {cat }} / \mathrm{hr}$, and 315 at $15,000 \mathrm{~L} / \mathrm{L}_{\text {cat }} / \mathrm{hr}$.

The catalyst was very reactive above $275^{\circ} \mathrm{C}$ and temperature control was difficult to attain when the catalyst temperature was first increased to approximately $300^{\circ} \mathrm{C}$ at a space velocity of $11,000 \mathrm{~L} / \mathrm{L}_{\text {cat }} / \mathrm{hr}$. The catalyst temperature initially reached an apparent steady-state value at approximately $311^{\circ} \mathrm{C}$ slowly cooling overnight to approximately $301^{\circ} \mathrm{C}$ for an average temperature of approximately $306^{\circ} \mathrm{C}$. However, there were several periods overnight when the catalyst experienced temperature spikes to approximately $330^{\circ} \mathrm{C}$ for periods of about 2 minutes, which may have caused some deactivation of the catalyst. The subsequent test condition of $300^{\circ} \mathrm{C}$ had very good temperature control $\left( \pm 1.5^{\circ} \mathrm{C}\right.$ about the average temperature). When the catalyst temperature was increased to the next condition, it was very difficult to control temperature again, and several temperature spikes to as high as $338^{\circ} \mathrm{C}$ were observed while slowly increasing the circulating oil temperature. After the catalyst temperature was stabilized at approximately $315^{\circ} \mathrm{C}$, the catalyst temperature was maintained within $\pm 2^{\circ} \mathrm{C}$ of the reported temperature. It was not possible to obtain a stable temperature at $325^{\circ} \mathrm{C}$ at a space velocity of $15,000 \mathrm{~L} / \mathrm{L}_{\text {cat }} / \mathrm{hr}$, with the catalyst temperature rapidly increasing to as high as $360^{\circ} \mathrm{C}$ while trying to attain a stable temperature. Decreasing the space velocity to $14,250 \mathrm{~L} / \mathrm{L}_{\text {cat }} / \mathrm{hr}$ produced an oscillation between 336 and $443^{\circ} \mathrm{C}$. Further decreasing the space velocity to $1350 \mathrm{~L} / \mathrm{L}_{\text {cat }} / \mathrm{hr}$ resulted in a temperature oscillation between 317 and $328^{\circ} \mathrm{C}$ over 2.5 hours. Finally the space velocity was returned to $11,000 \mathrm{~L} / \mathrm{L}_{\text {cat }} / \mathrm{hr}$, and the oil temperature was adjusted in an attempt to attain a stable temperature at $300^{\circ} \mathrm{C}$. Instead, the temperature slowly increased from approximately 298 to $313^{\circ} \mathrm{C}$ over a 14 -hour period where it remained at $313^{\circ} \mathrm{C}$ for 
the remainder of the sampling period. The average temperature for the entire sampling period was $307^{\circ} \mathrm{C}$ with a $+6^{\circ} \mathrm{C}$ to $-8^{\circ} \mathrm{C}$ variation about the reported temperature. The last test condition was obtained by increasing the space velocity to $15,000 \mathrm{~L} / \mathrm{L}_{\text {cat }} / \mathrm{hr}$. while maintaining the oil temperature. The catalyst responded by increasing its temperature to approximately $313 \pm 1^{\circ} \mathrm{C}$ where it remained throughout the sampling period.

Figure B.49 shows the carbon conversion for the various conditions during the test. It can be seen that carbon conversion increases with temperature and decreases with space velocity as expected. It also appears that the temperature excursion that took place while attempting to raise the catalyst temperature to $325^{\circ} \mathrm{C}$ caused some deactivation of the catalyst as evidenced by the decrease in carbon conversion for the repeat condition at $307^{\circ} \mathrm{C}$. The reduced $\mathrm{C}_{2}+$-oxygenate STY for the repeat condition as well as the subsequent condition at approximately $315^{\circ} \mathrm{C}$ and a $15,000 \mathrm{~L} / \mathrm{L}_{\text {cat }} / \mathrm{hr}$ space velocity also suggests some deactivation of the catalyst as shown in Figure B.50. In the latter case, increasing the space velocity to lower carbon conversion from $40 \%$ to a lower value was expected to result in a higher STY than that obtained previously at $315^{\circ} \mathrm{C}$ and $11,000 \mathrm{~L} / \mathrm{L}_{\text {cat }} / \mathrm{hr}$. The highest $\mathrm{C}_{2}+$-oxygenate STY obtained at $315^{\circ} \mathrm{C}$ and $11,000 \mathrm{~L} / \mathrm{L}_{\text {cat }} / \mathrm{hr}$ was $664 \mathrm{~g} / \mathrm{L}_{\text {cat }} / \mathrm{hr}$.

Figure B.51 shows that the carbon selectivity to oxygenates gradually decreased with increasing temperature and did not appear to be sensitive to the space velocity. However, they may be evidence of an increase in selectivity to oxygenates at temperatures above $310^{\circ} \mathrm{C}$ although the effect is relatively minor with selectivity still below $40 \%$ under these conditions. There is no evidence that deactivation of the catalyst effected the selectivity to $\mathrm{C}_{2}+$ oxygenates. Figure B.52 shows an increase in the ratio of $\mathrm{C}_{2}+$ alcohols to all oxygenates with increasing temperature that also does not appear to be sensitive to the space velocity. Deactivation of the catalyst may have resulted in a minor improvement in the selectivity of the oxygenates to $\mathrm{C}_{2}+$ alcohols as evidenced by the selectivity for the repeat conditions. Figure B.53 shows that deactivation of the catalyst above $300^{\circ} \mathrm{C}$ resulted in reduced selectivity of the hydrocarbon liquids although they were not eliminated as was the case for many of the other catalysts. 


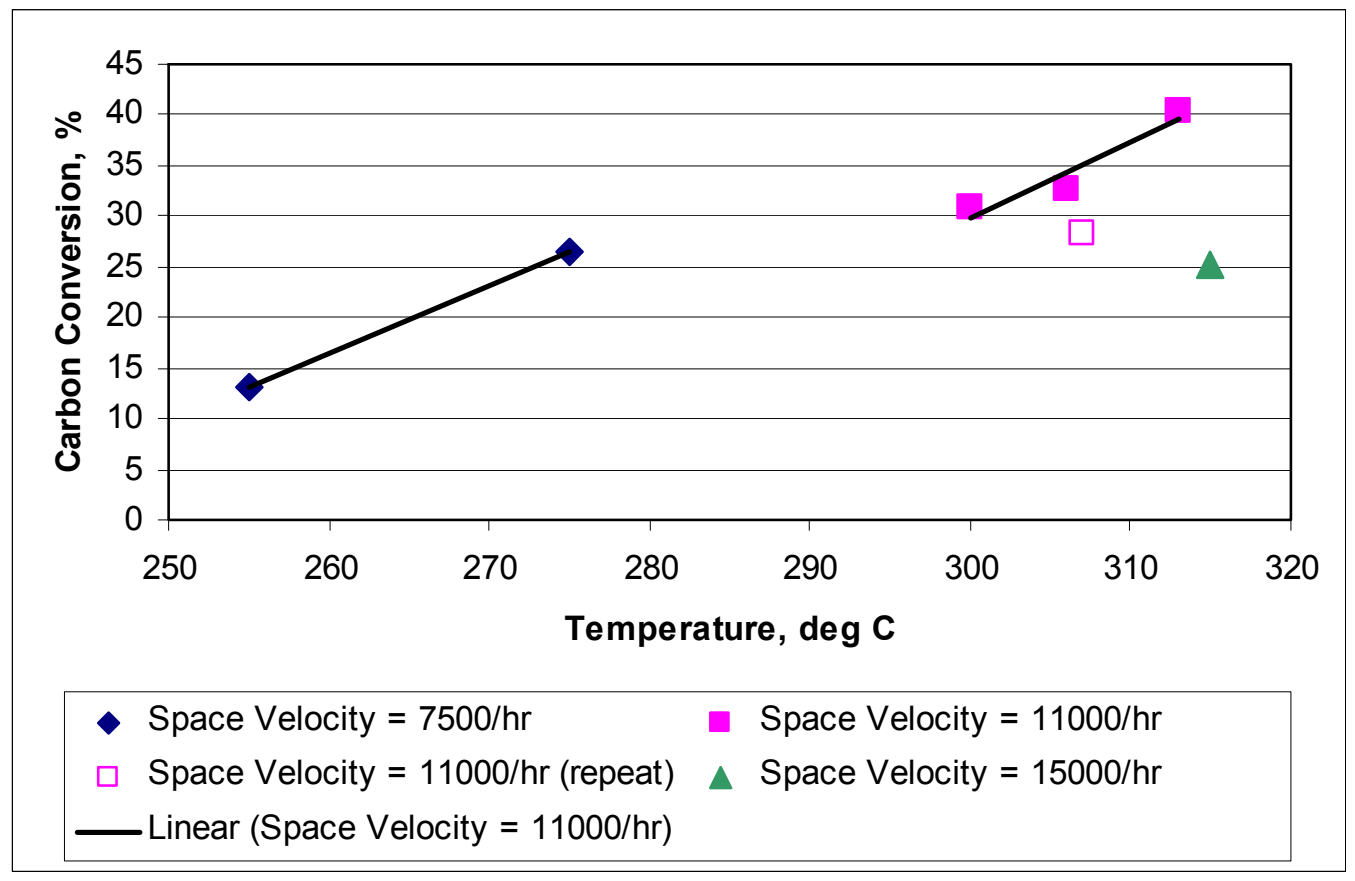

Figure B.49. Carbon Conversion for the Pt-Promoted Catalyst

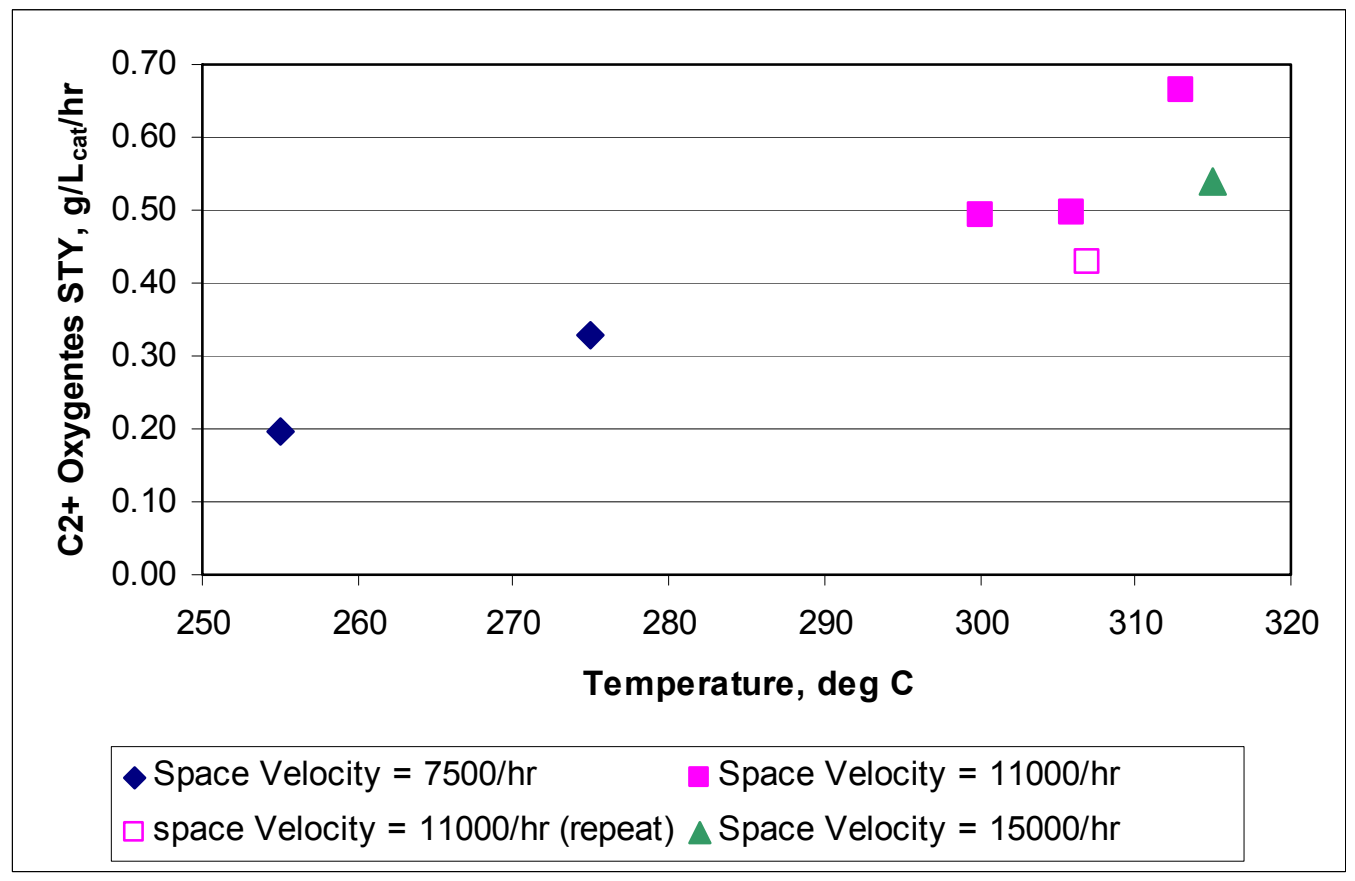

Figure B.50. $\mathrm{C}_{2}+-$-Oxygenate STYs for the Pt-Promoted Catalyst 


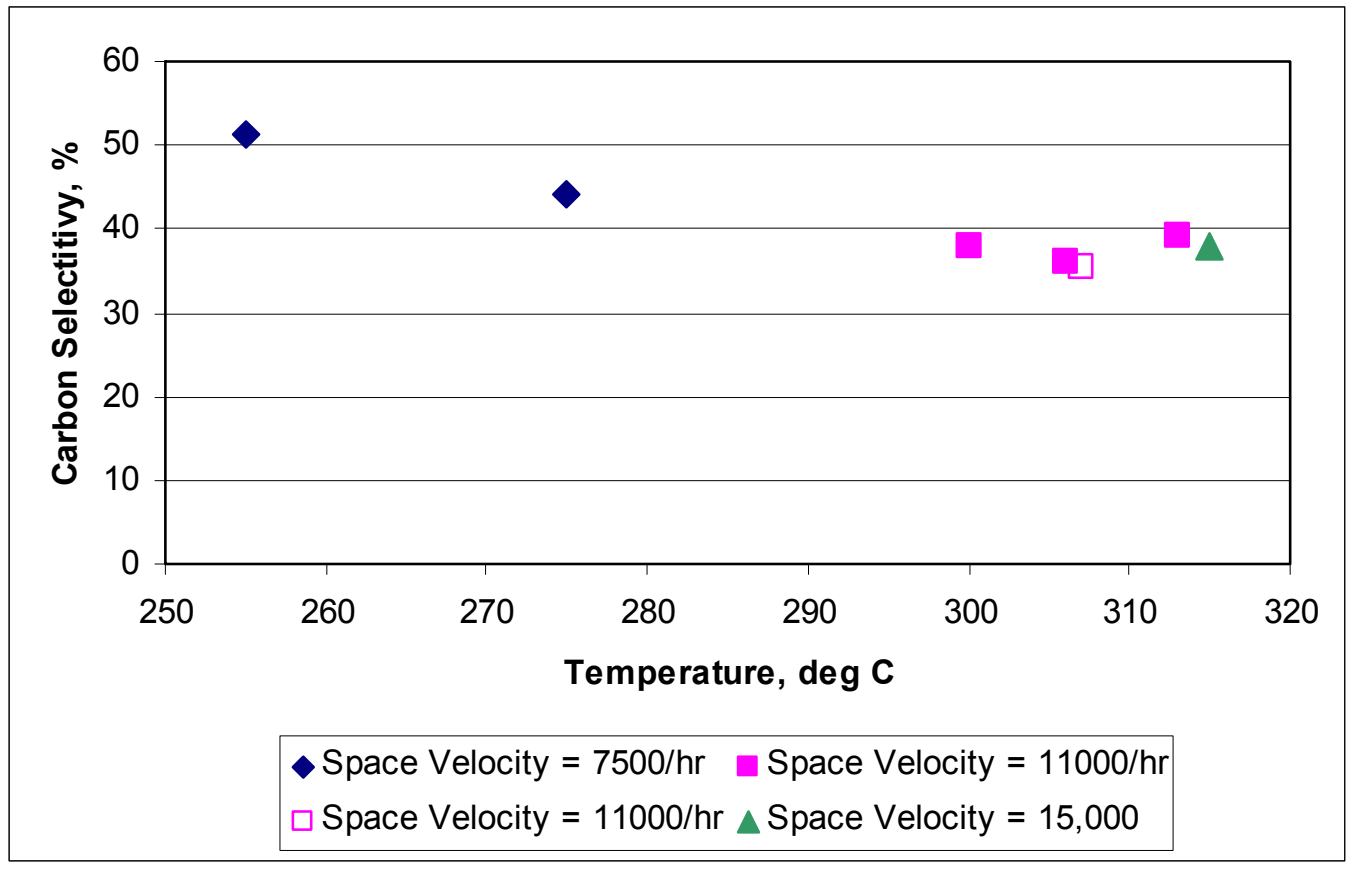

Figure B.51. Converted Carbon Selectivity to $\mathrm{C}_{2}+$ Oxygenates for the Pt-Promoted Catalyst

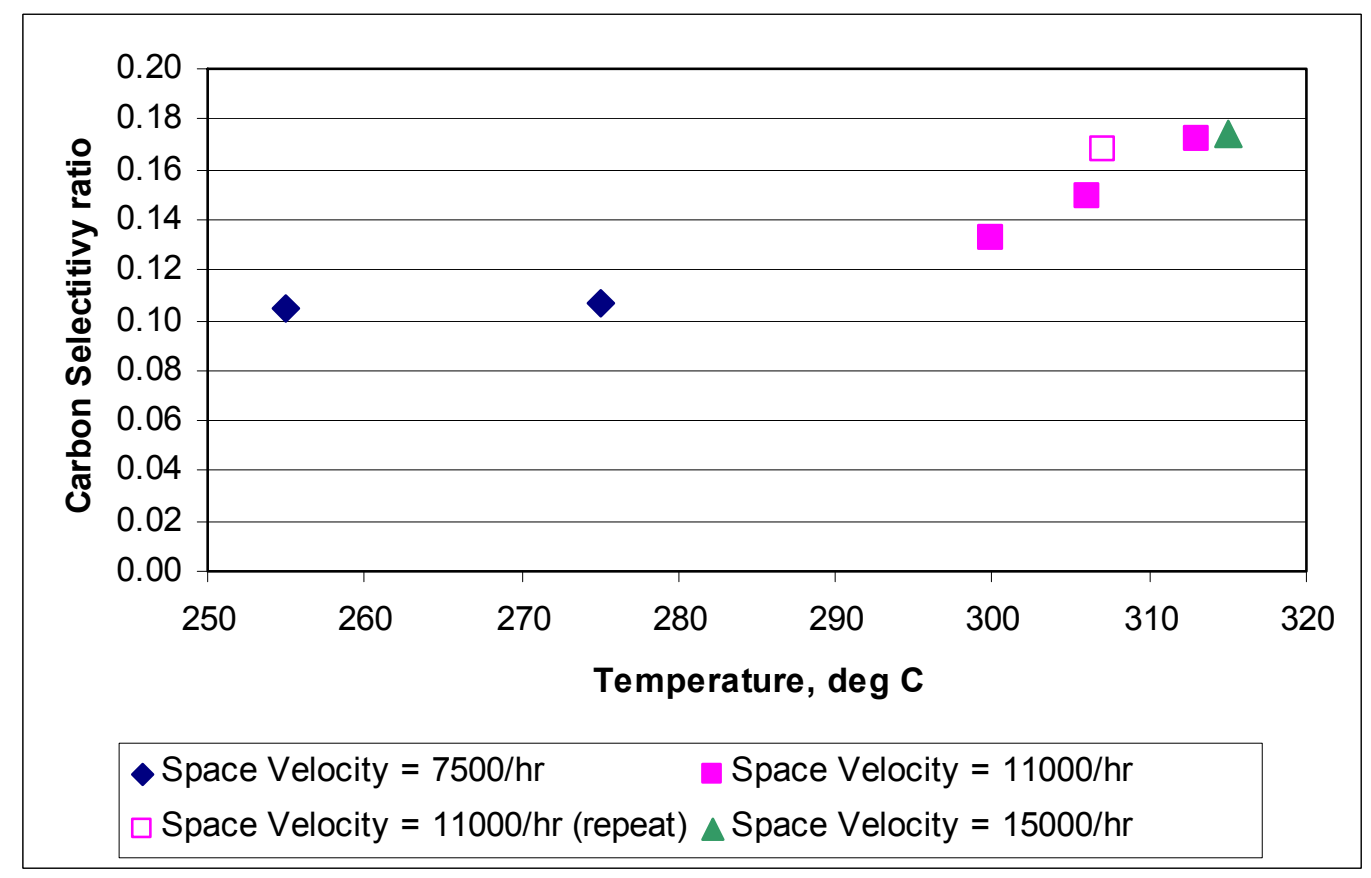

Figure B.52. Carbon Selectivity of All Oxygenates to $\mathrm{C}_{2}+$ Alcohols for the Pt-Promoted Catalyst 


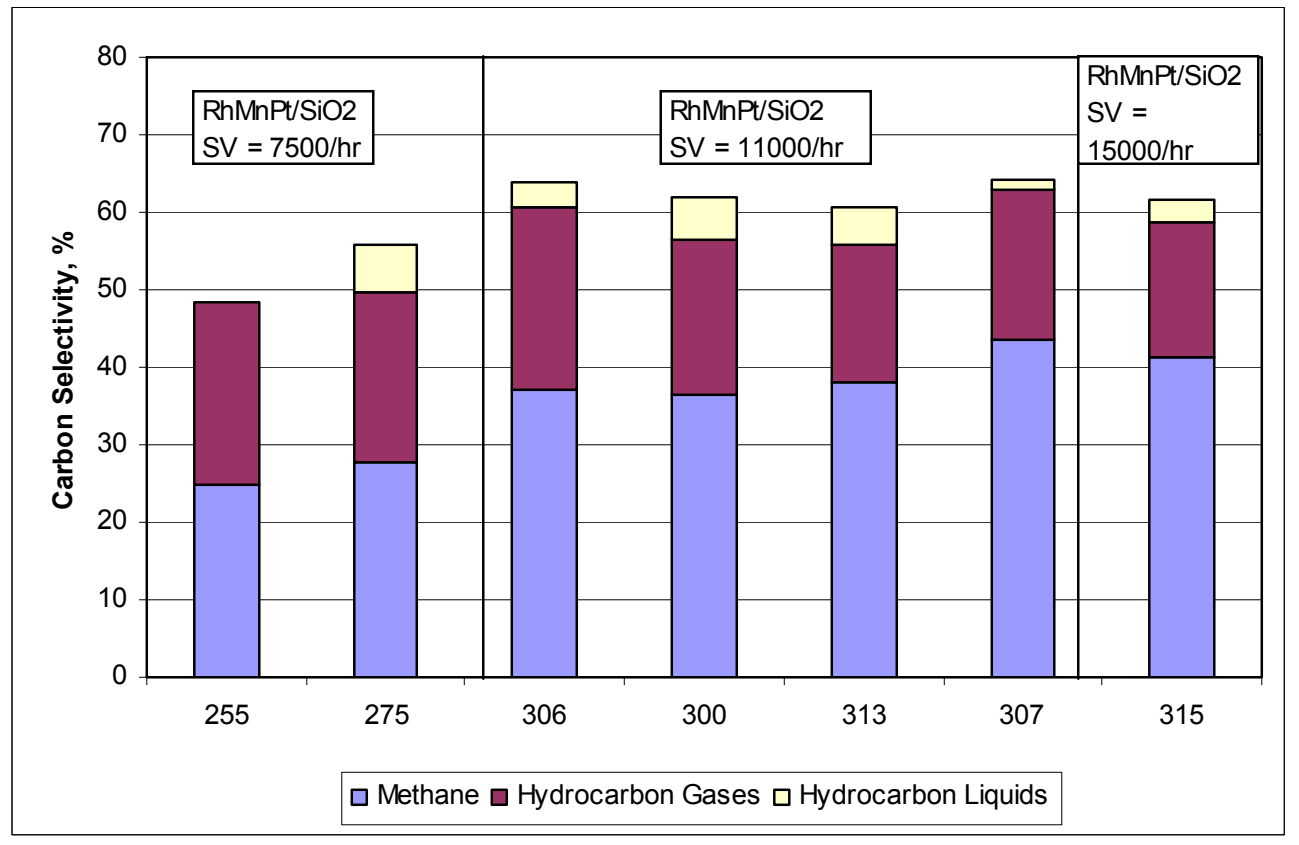

Figure B.53. Comparison of Hydrocarbon Selectivity for the Pt-Promoted Catalyst

\section{B.12 Ru-Promoted Catalyst}

The Ru-promoted catalyst $\left(\mathrm{RhMnRu} / \mathrm{SiO}_{2}\right)$ was reduced at the maximum temperature of $350^{\circ} \mathrm{C}$ and tested using the hot oil circulating system to heat the reactor. The catalyst testing sequence was 256 and $274^{\circ} \mathrm{C}$ at 7500 at L/L $/ \mathrm{L}_{\text {cat }} / \mathrm{hr} ; 300,313$, and $323^{\circ} \mathrm{C}$ at $11,000 \mathrm{~L} / \mathrm{L}_{\text {cat }} / \mathrm{hr} ; 325$ and $302^{\circ} \mathrm{C}$ at $15,000 \mathrm{~L} / \mathrm{L}_{\text {cat }} / \mathrm{hr}$; and finally a repeat condition of $303^{\circ} \mathrm{C}$ at $11,000 \mathrm{~L} / \mathrm{L}_{\text {cat }} / \mathrm{hr}$. The temperature control was generally good with the catalyst varying $\pm 1.5^{\circ} \mathrm{C}$ for most test conditions. However, the temperature decreased much more over the sampling time for the test conditions at 300 and $313^{\circ} \mathrm{C}$ at $11,000 \mathrm{~L} / \mathrm{L}_{\mathrm{cat}} / \mathrm{hr}$, with temperatures ranging \pm approximately 3.4 and $2.5^{\circ} \mathrm{C}$, respectively, about the reported temperatures.

It appears that the catalyst underwent significant deactivation at $300^{\circ} \mathrm{C}$ or higher, as indicated by the decreasing carbon conversion with temperature for the catalyst at 300,313 , and $323^{\circ} \mathrm{C}$ at $11,000 \mathrm{~L} / \mathrm{L}_{\text {cat }} / \mathrm{hr}$, and the repeat condition at $303^{\circ} \mathrm{C}$ at $11,000 \mathrm{~L} / \mathrm{L}_{\text {cat }} / \mathrm{hr}$ as shown in Figure B.54. This deactivation behavior was also evidenced in the STY's for the catalyst over the same set of temperatures and space velocities as shown in Figure B.55. So, while a maximum $\mathrm{C}_{2}+$-oxygenate $\mathrm{STY}$ of $440 \mathrm{~g} / \mathrm{L}_{\text {cat }} / \mathrm{hr}$ was achieved at $300^{\circ} \mathrm{C}$ and $11,000 \mathrm{~L} / \mathrm{L}_{\text {cat }} / \mathrm{hr}$ space velocity, it could not be sustained regardless of attempts to do so by increasing the temperature and space velocity. Rather, the STY decreased by nearly $45 \%$ as indicated by the STY for the repeat conditions of $303^{\circ} \mathrm{C}$ and $11,000 \mathrm{~L} / \mathrm{L}_{\text {cat }} / \mathrm{hr}$ space velocity. Figure B.56 shows that the carbon selectivity to $\mathrm{C}_{2}+$ oxygenates was relatively low with a maximum of approximately $36 \%$ at $256^{\circ} \mathrm{C}$ and decreasing to approximately $20 \%$ at $325^{\circ} \mathrm{C}$. Similarly, the selectivity of all oxygenates to $\mathrm{C}_{2}+$ alcohols was very low, reaching a selectivity ratio of approximately $35 \%$ at $325^{\circ} \mathrm{C}$ as shown in Figure B.57. Figure B.58 shows that early in the test, the catalyst produced mainly hydrocarbons, with a mixture of methane, higher hydrocarbon gases, and hydrocarbon liquids, and with the products shifting to mostly methane and lesser amounts of higher hydrocarbon gases as the catalyst deactivated. 


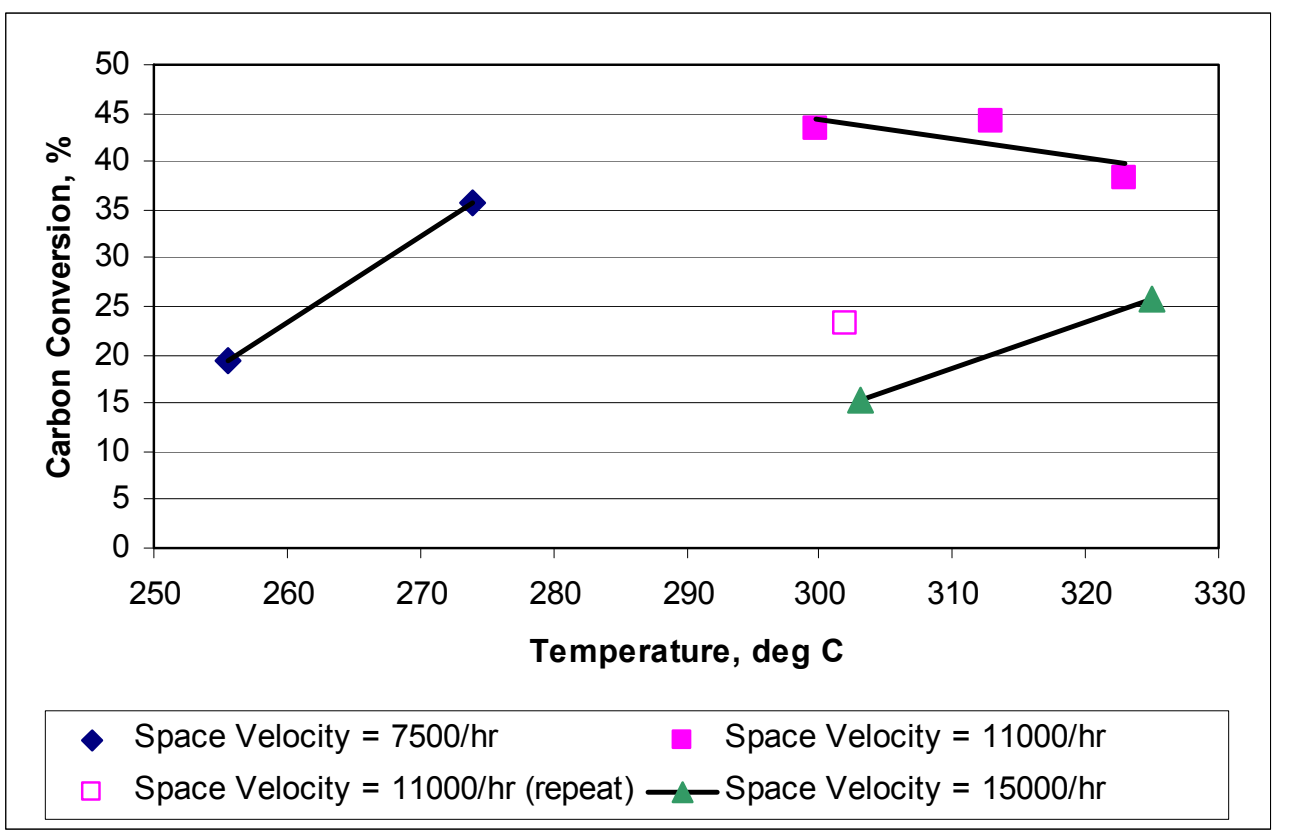

Figure B.54. Carbon Conversion for the Ru-Promoted Catalyst

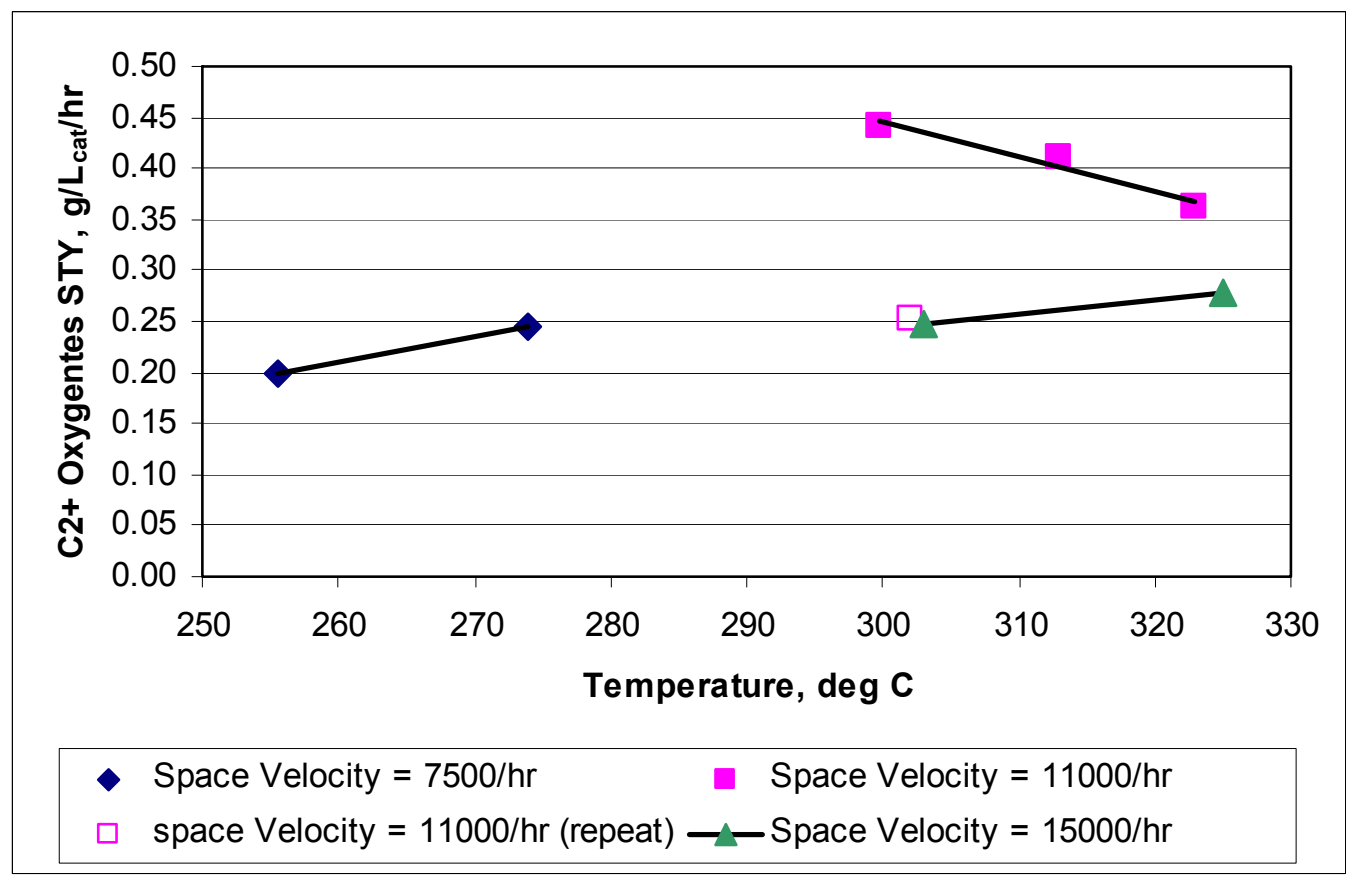

Figure B.55. $\mathrm{C}_{2}+-$ Oxygenate STYs for the Ru-Promoted Catalyst 


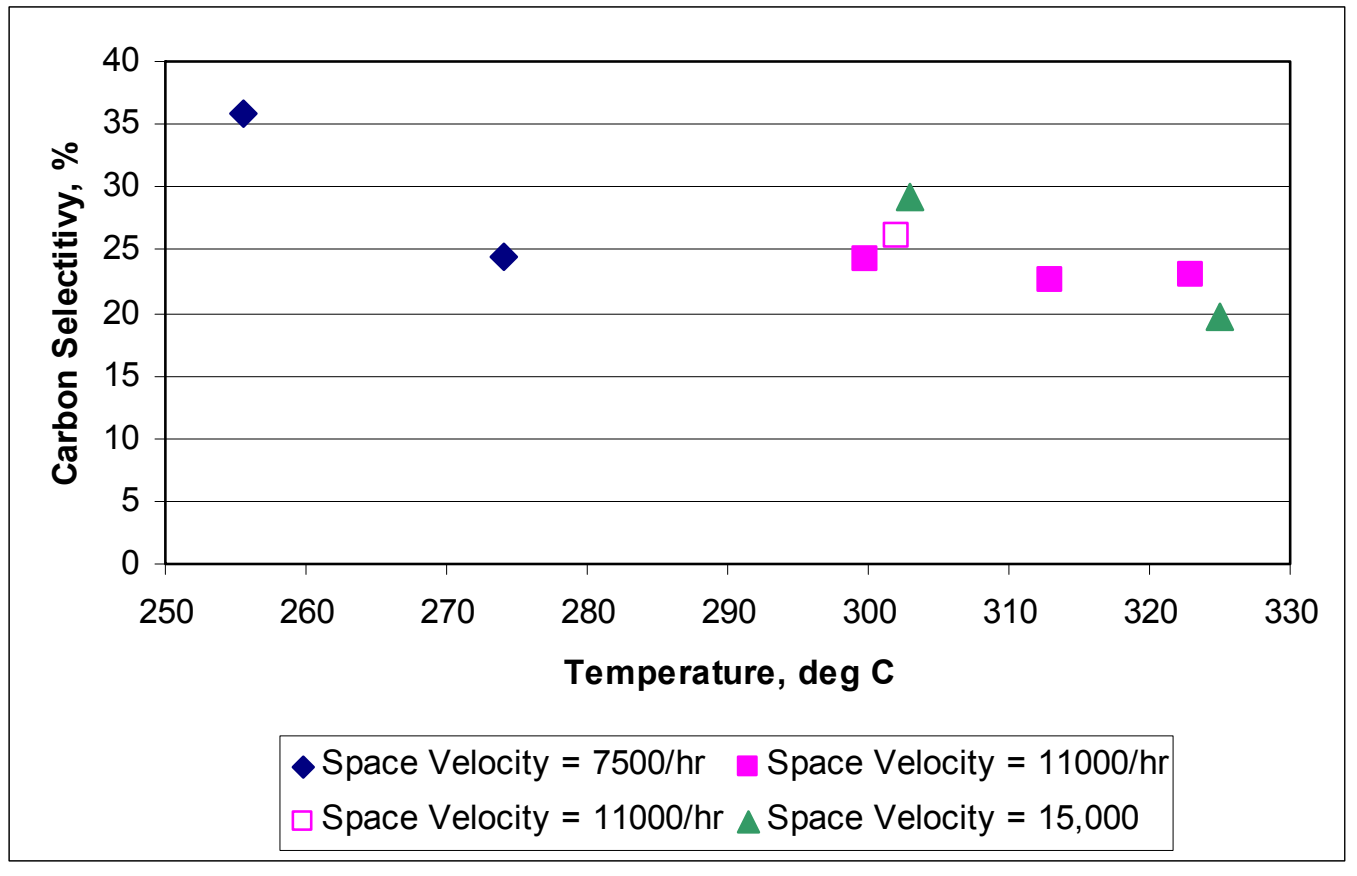

Figure B.56. Converted Carbon Selectivity to $\mathrm{C}_{2}+$ Oxygenates for the Ru-Promoted Catalyst

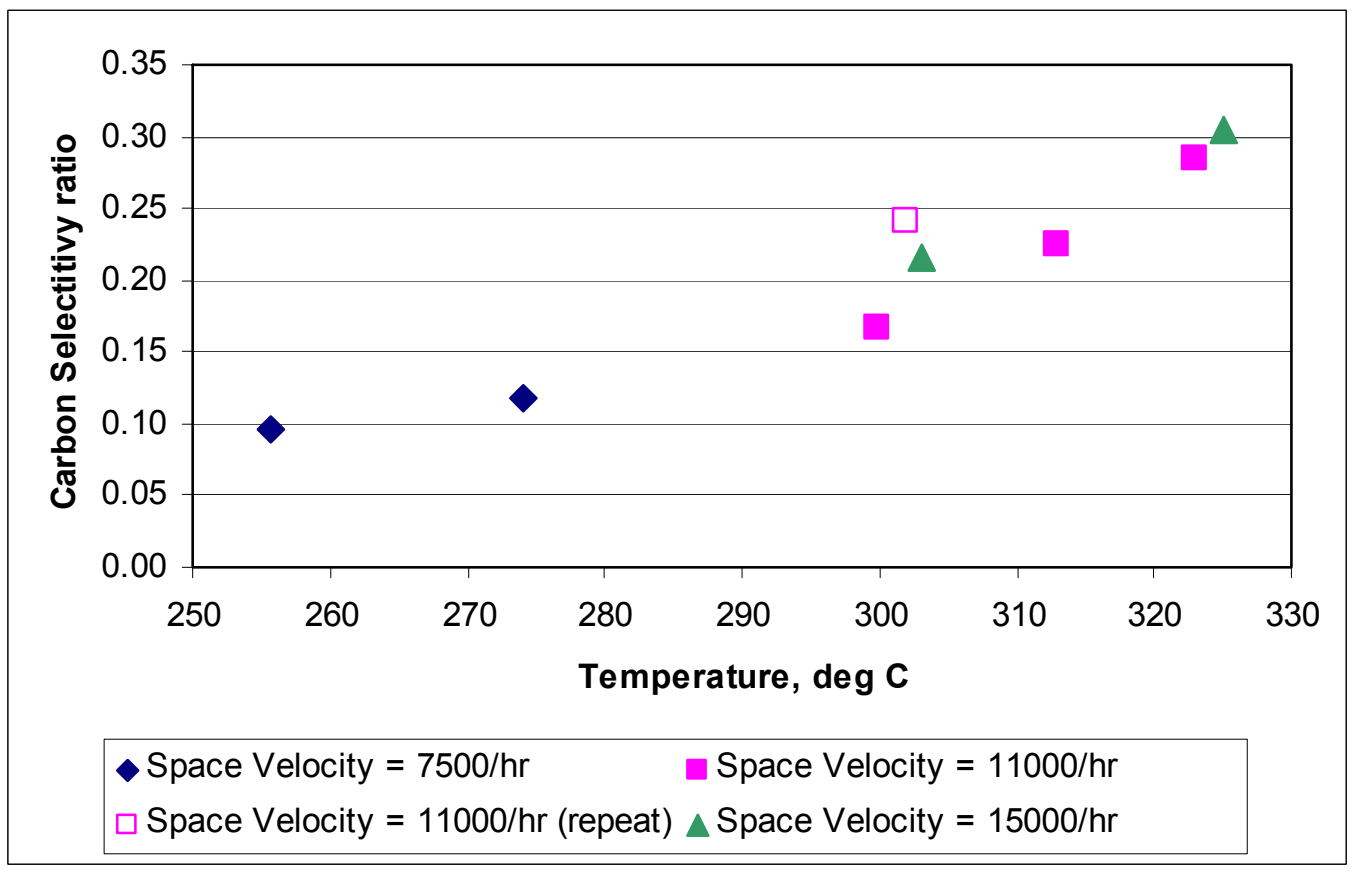

Figure B.57. Carbon Selectivity of All Oxygenates to $\mathrm{C}_{2}+$ Alcohols for the Ru-Promoted Catalyst 


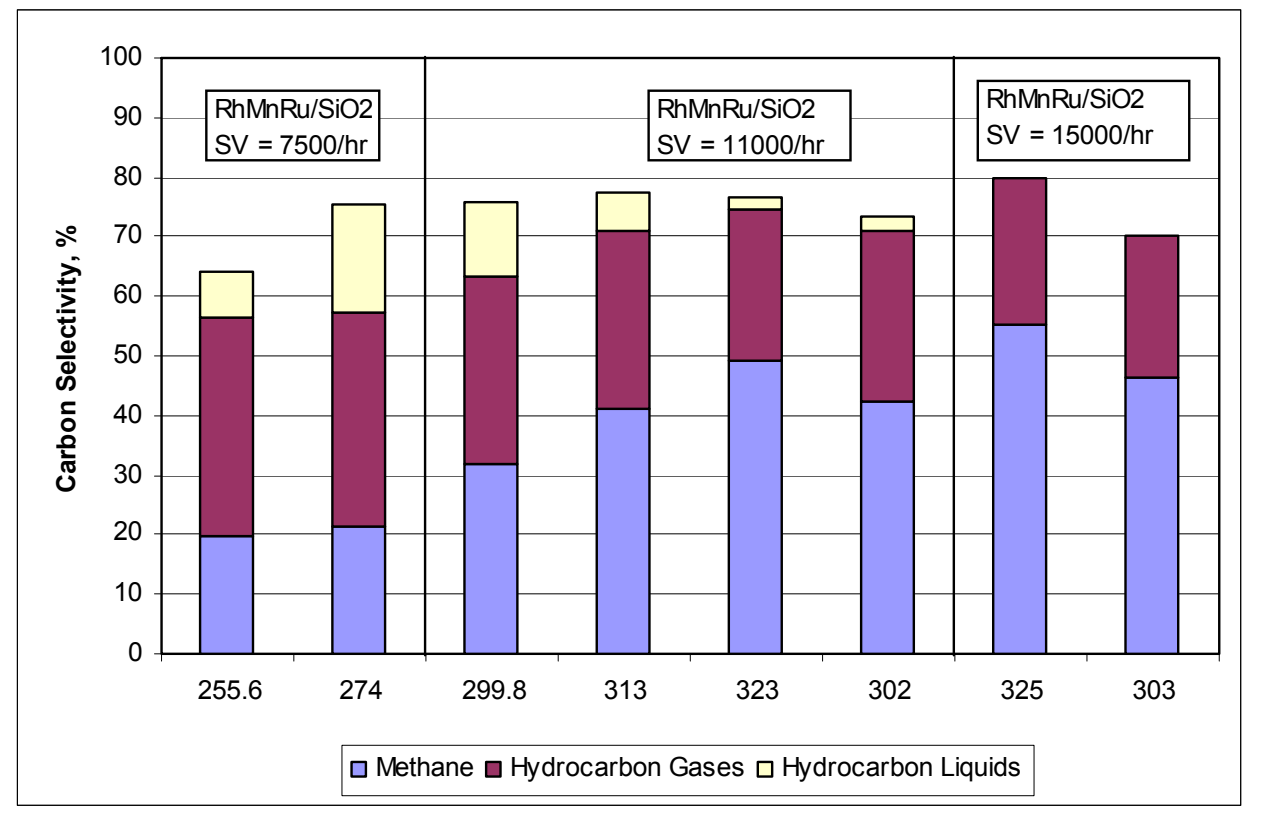

Figure B.58. Comparison of Hydrocarbon Selectivity for the Ru-Promoted Catalyst

\section{B.13 Ga-Promoted Catalyst}

The Ga-promoted catalyst $(\mathrm{RhMnGa} / \mathrm{SiO} 2)$ was reduced at the maximum temperature of $350^{\circ} \mathrm{C}$ and tested using the hot oil circulating system to heat the reactor. The catalyst testing sequence was 255 and $275^{\circ} \mathrm{C}$ at 7500 at $\mathrm{L} / \mathrm{L}_{\text {cat }} / \mathrm{hr}$, and 300,324 , and $345^{\circ} \mathrm{C}$ at $11,000 \mathrm{~L} / \mathrm{L}_{\text {cat }} / \mathrm{hr}$. The temperature control was very good with the catalyst varying $\pm 1.0^{\circ} \mathrm{C}$ for all test conditions.

The Ga-promoted catalysts had a relatively low activity, achieving a maximum carbon conversion of only $18 \%$ at a catalyst temperature of $345^{\circ} \mathrm{C}$ at a space velocity of $11,000 \mathrm{~L} / \mathrm{L}_{\text {cat }} / \mathrm{hr}$, as shown in Figure B.59. This can be compared to the carbon conversion of approximately $25 \%$ at $314^{\circ} \mathrm{C}$ for the $\mathrm{RhMn} / \mathrm{SiO}_{2}$ * catalyst that was prepared using the same (Fisher Scientific) source of $\mathrm{SiO}_{2}$ (Figure B.1). Interestingly, it appears that the catalyst activity improved at temperatures above approximately $300^{\circ} \mathrm{C}$. As might be expected, the $\mathrm{C}_{2}+$-oxygenate STYs also were relatively low, reaching a maximum of approximately $190 \mathrm{~g} / \mathrm{L}_{\text {cat }} / \mathrm{hr}$ at $345^{\circ} \mathrm{C}$ and a space velocity of $11,000 \mathrm{~L} / \mathrm{L}_{\text {cat }} / \mathrm{hr}$ as shown in Figure B.60, and it was much lower than that achieved using the unpromoted catalyst prepared from the same source of $\mathrm{SiO}_{2}$ (Figure B.2). Although no repeat conditions were evaluated, the Ga-promoted catalyst did not show any clear evidence of deactivation over the course of the experiment. Figure B.61 shows that the carbon selectivity to $\mathrm{C}_{2}+$ oxygenates for the Ga-promoted catalyst decreased with increasing temperature as expected, but there was clearly an improvement in the selectivity for reaction temperatures at or above $300^{\circ} \mathrm{C}$. The selectivity of all oxygenates to $\mathrm{C}_{2}+$ alcohols was very high compared to other catalysts, and remained relatively constant at 65 to $70 \%$ over the course of the experiment as shown in Figure B.62. Furthermore, selectivity of the oxygenates to all $\left(\mathrm{C}_{1}+\right)$ alcohols was constant at 86 to $88 \%$ over the course of the experiment. This may be attributed, at least in part to the source of $\mathrm{SiO}_{2}$ used to prepare this catalyst, which also resulted in significant methanol production for the unpromoted $\mathrm{RhMn} / \mathrm{SiO}_{2}$ * catalyst (see Figure B.5). Figure B.63 shows that the Ga-promoted catalyst did not produce liquid hydrocarbons, even at $345^{\circ} \mathrm{C}$. Furthermore, the fraction of higher hydrocarbon gases decreased between 225 and $345^{\circ} \mathrm{C}$, suggesting some deactivation of the catalyst with respect to hydrocarbon chain growth. 


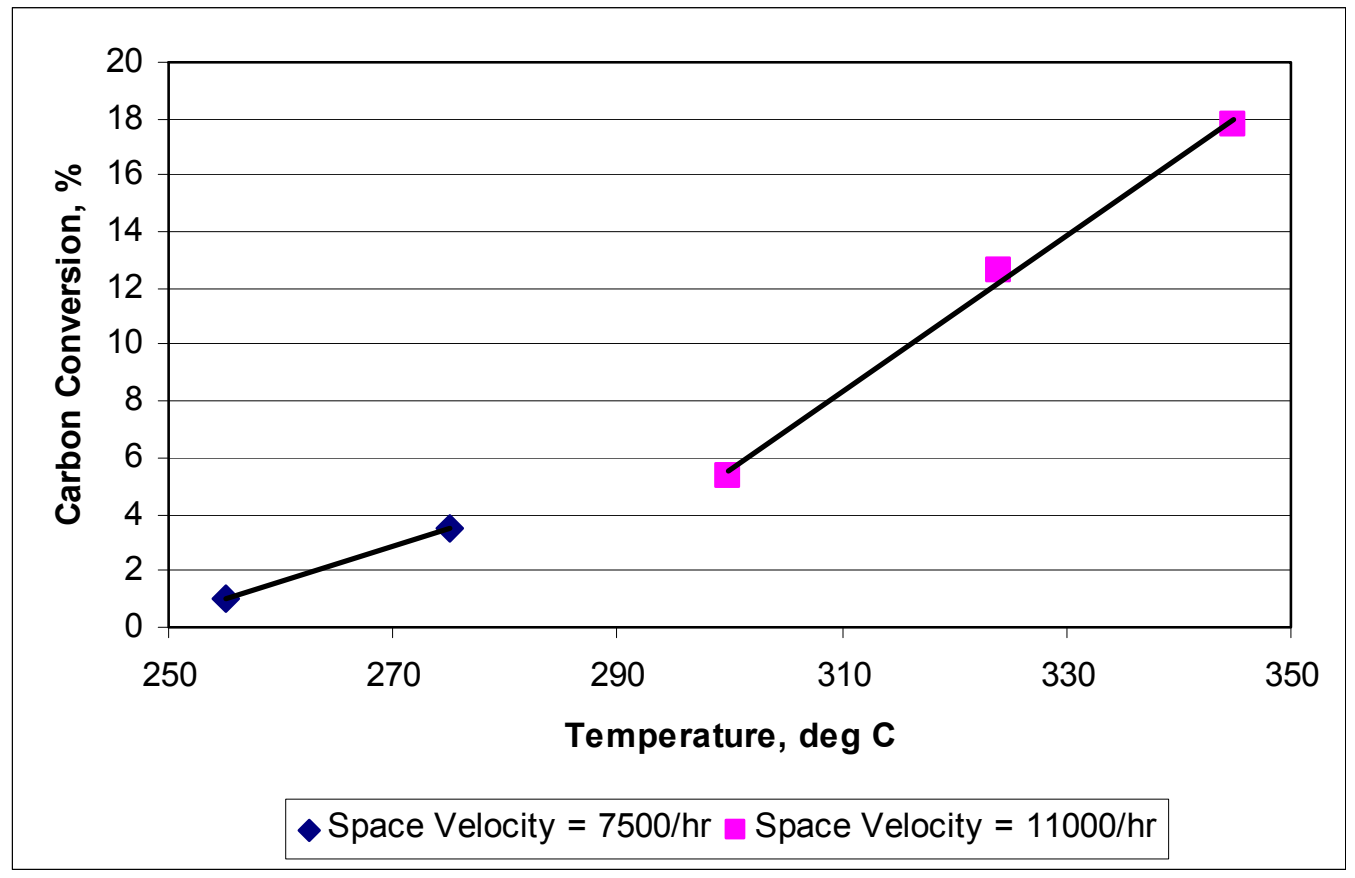

Figure B.59. Carbon Conversion for the Ga Promoted Catalyst

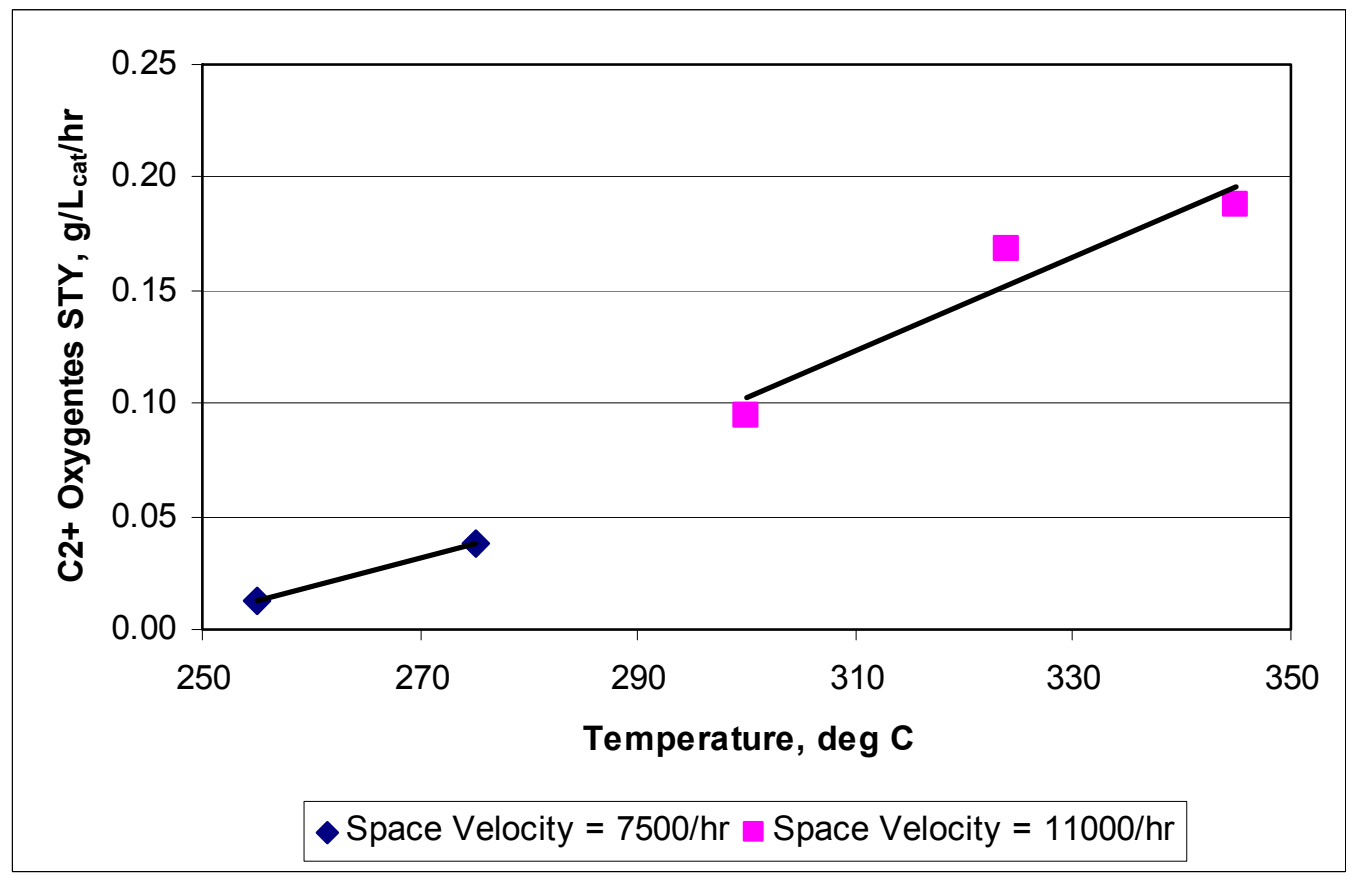

Figure B.60. $\mathrm{C}_{2}+-$ Oxygenate STYs for the Ga-Promoted Catalyst 


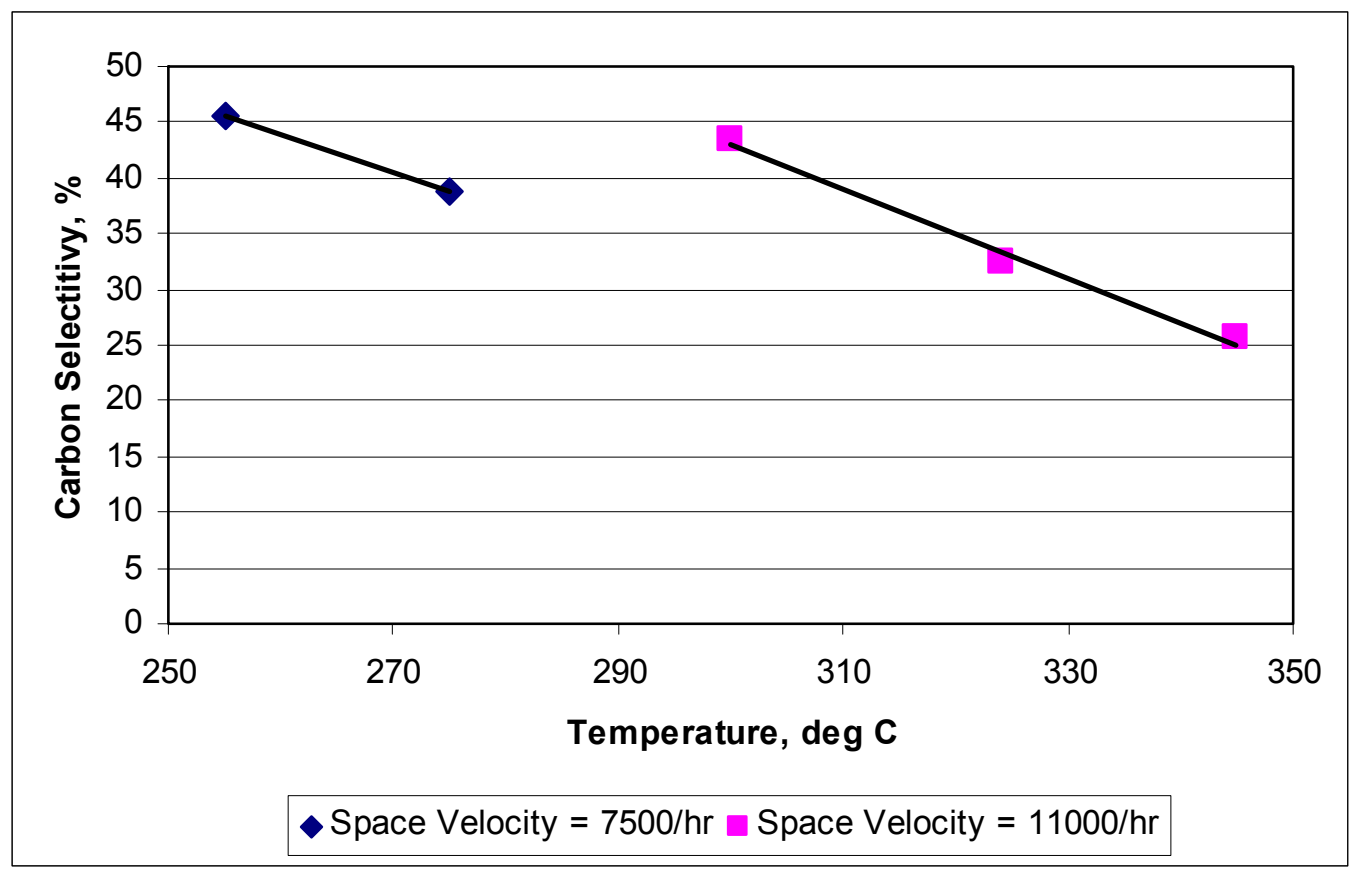

Figure B.61. Converted Carbon Selectivity to $\mathrm{C}_{2}+$ Oxygenates for the Ga-Promoted Catalyst

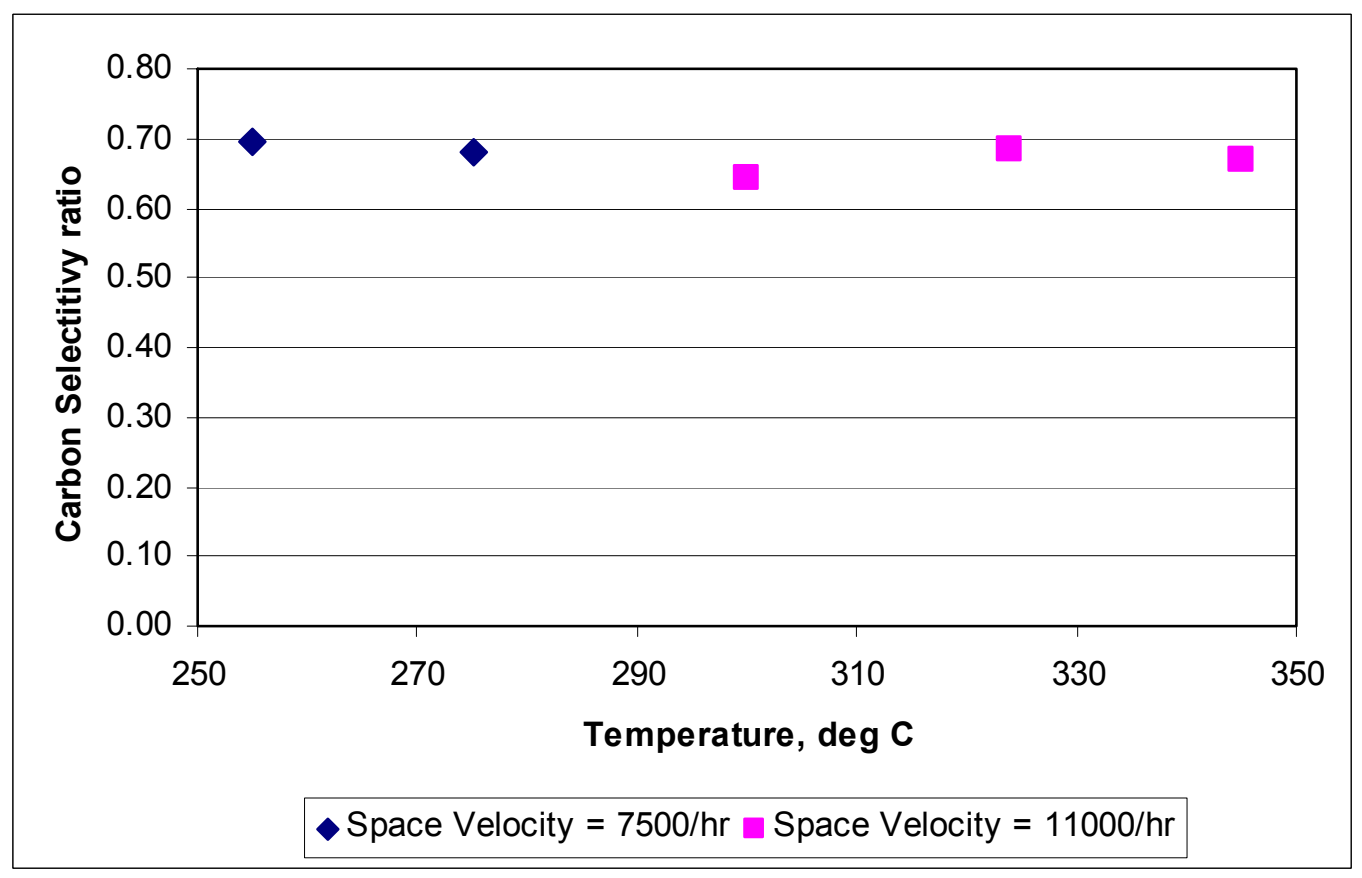

Figure B.62. Carbon Selectivity of All Oxygenates to $\mathrm{C}_{2}+$ Alcohols for the Ga-Promoted Catalyst 


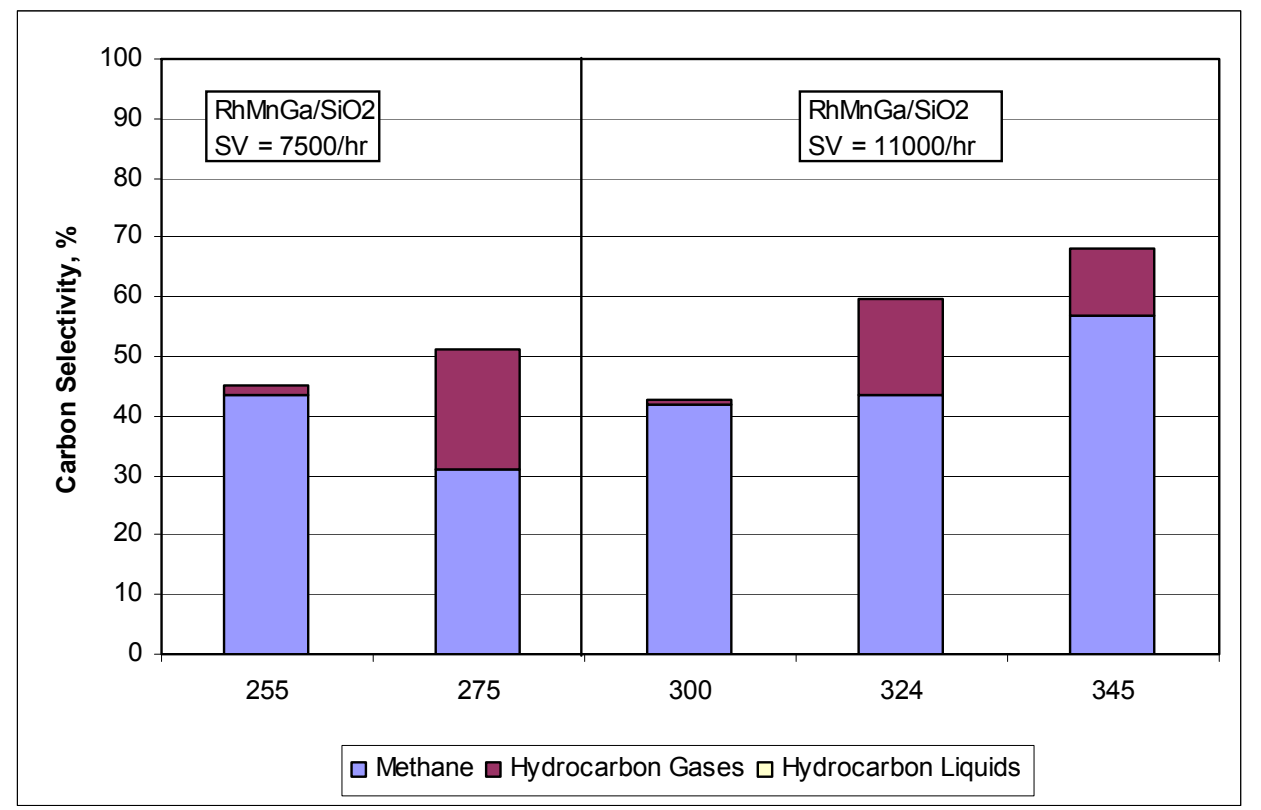

Figure B.63. Comparison of Hydrocarbon Selectivity for the Ga-Promoted Catalyst

\section{B.14 In-Promoted Catalyst}

The In-promoted catalyst $\left(\mathrm{RhMnIn} / \mathrm{SiO}_{2}\right)$ catalyst was reduced at the maximum temperature of $350^{\circ} \mathrm{C}$ and tested using the hot oil circulating system to heat the reactor. The catalyst testing sequence was 256 and $276^{\circ} \mathrm{C}$ at 7500 at $\mathrm{L} / \mathrm{L}_{\text {cat }} / \mathrm{hr}$, and 300,325 , and $345^{\circ} \mathrm{C}$ at $11,000 \mathrm{~L} / \mathrm{L}_{\text {cat }} / \mathrm{hr}$. The temperature control was very good with the catalyst varying $\pm 1.0^{\circ} \mathrm{C}$ for all test conditions.

The In-promoted catalyst behaved similarly to the Ga-promoted catalyst, except that it was less reactive.

The In-promoted catalyst achieved maximum carbon conversion of only $9 \%$ at a catalyst temperature of $345^{\circ} \mathrm{C}$ at a space velocity of $11,000 \mathrm{~L} / \mathrm{L}_{\mathrm{cat}} / \mathrm{hr}$, as shown in Figure B.64, which was about one-half of that achieved with the Ga-promoted catalyst. The In-promoted catalyst activity also improved above approximately $300^{\circ} \mathrm{C}$. Figure B. 65 shows that the $\mathrm{C}_{2}+$-oxygenate STY reached a maximum of approximately $140 \mathrm{~g} / \mathrm{L}_{\text {cat }} / \mathrm{hr}$ at $345^{\circ} \mathrm{C}$ and a space velocity of $11,000 \mathrm{~L} / \mathrm{L}_{\text {cat }} / \mathrm{hr}$. Although no repeat conditions were evaluated, the In-promoted catalyst did not show any clear evidence of deactivation over the course of the experiment. Figure B.66 shows that the carbon selectivity to $\mathrm{C}_{2}+$ oxygenates for the In-promoted catalyst initially decreased from approximately $65 \%$ at $256^{\circ} \mathrm{C}$ to $40 \%$ at $276^{\circ} \mathrm{C}$, but then remained relatively constant between 33 and $42 \%$ from 300 to $345^{\circ} \mathrm{C}$. The selectivity of all oxygenates to $\mathrm{C}_{2}+$ oxygenates was very high, like the Ga-promoted catalyst, increasing from $45 \%$ at $256^{\circ} \mathrm{C}$ to a relatively constant range between 67 and $73 \%$ over remainder of the experiment as shown in Figure B.67. Selectivity of the oxygenates to all $\left(\mathrm{C}_{1}+\right)$ alcohols increased from $56 \%$ at $256^{\circ} \mathrm{C}$ to a relatively constant value 90 to $93 \%$ over the remainder of the experiment. Again this may be attributed, at least in part to the source of $\mathrm{SiO}_{2}$ used to prepare this catalyst, which also resulted in significant methanol production for the unpromoted $\mathrm{RhMn} / \mathrm{SiO}_{2}{ }^{*}$ catalyst (see Figure B.5). Figure B.68 shows that, like the Ga-promoted catalyst, the In-promoted catalyst did not produce liquid hydrocarbons, even at $345^{\circ} \mathrm{C}$. Furthermore the fraction of higher hydrocarbon gases decreased between 276 and $300^{\circ} \mathrm{C}$ and between 325 and $345^{\circ} \mathrm{C}$, suggesting some deactivation with respect to hydrocarbon chain growth. 


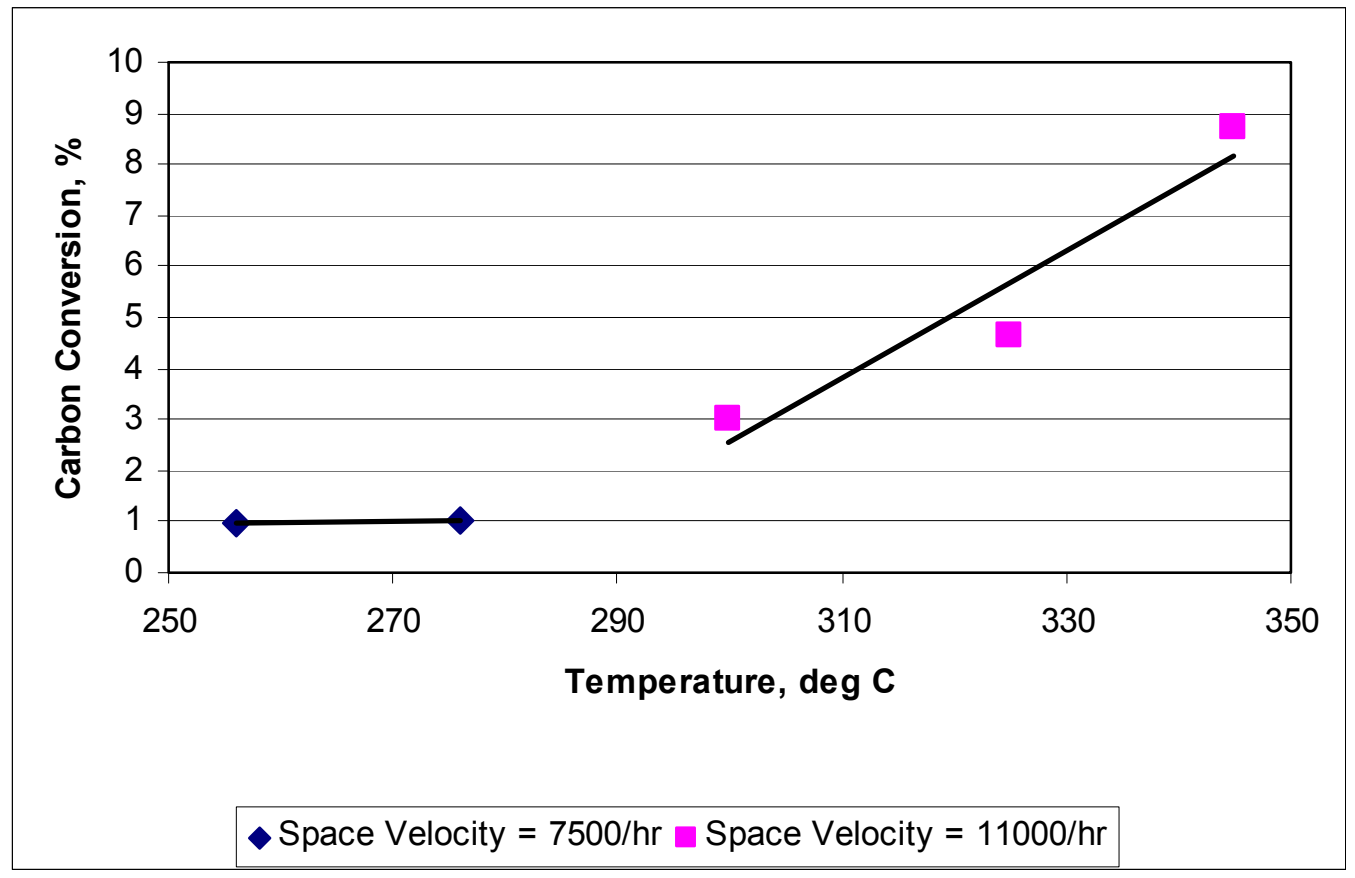

Figure B.64. Carbon Conversion for the In-Promoted Catalyst

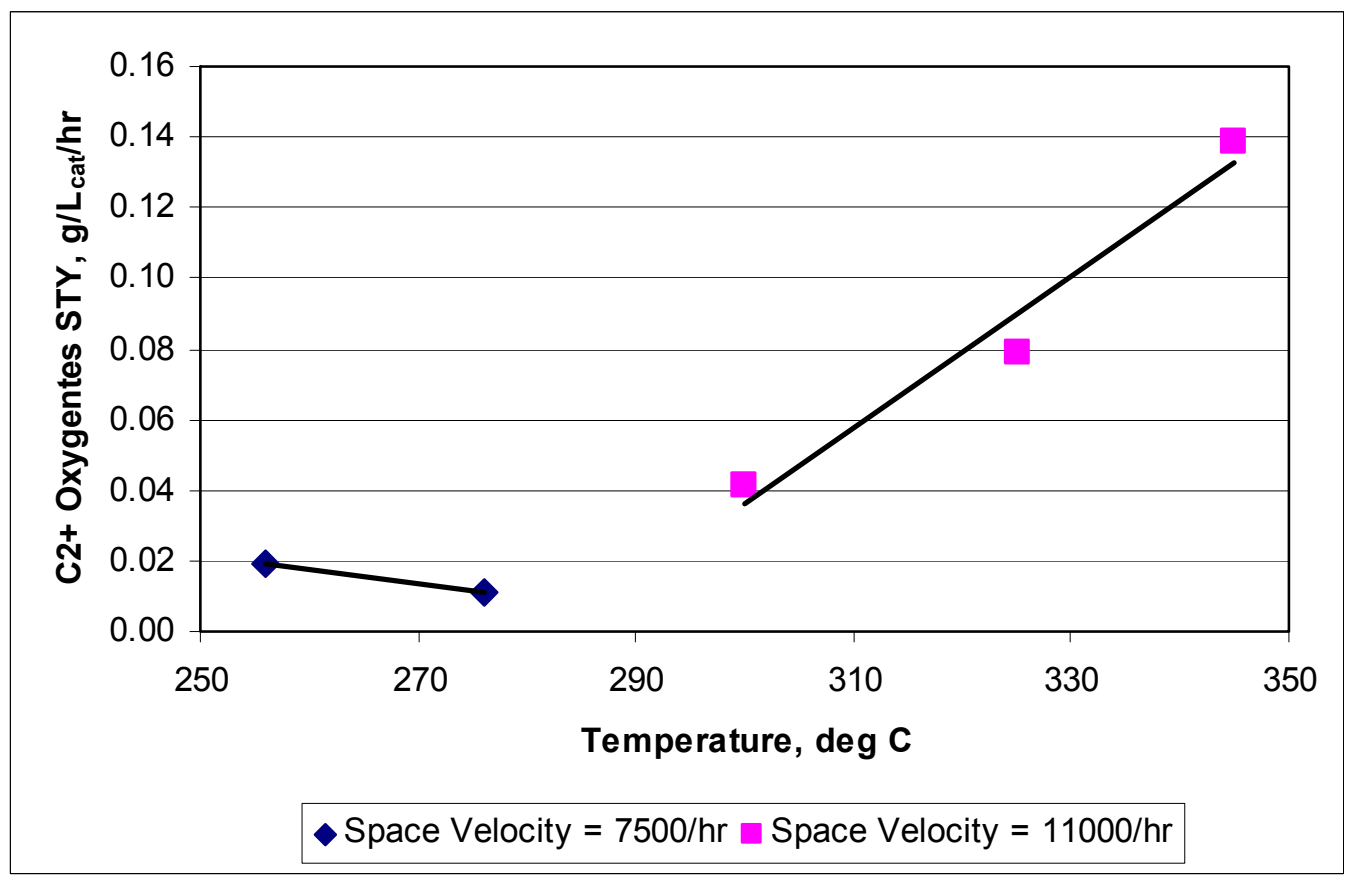

Figure B.65. $\mathrm{C}_{2}+-$-Oxygenate STYs for the In-Promoted Catalyst 


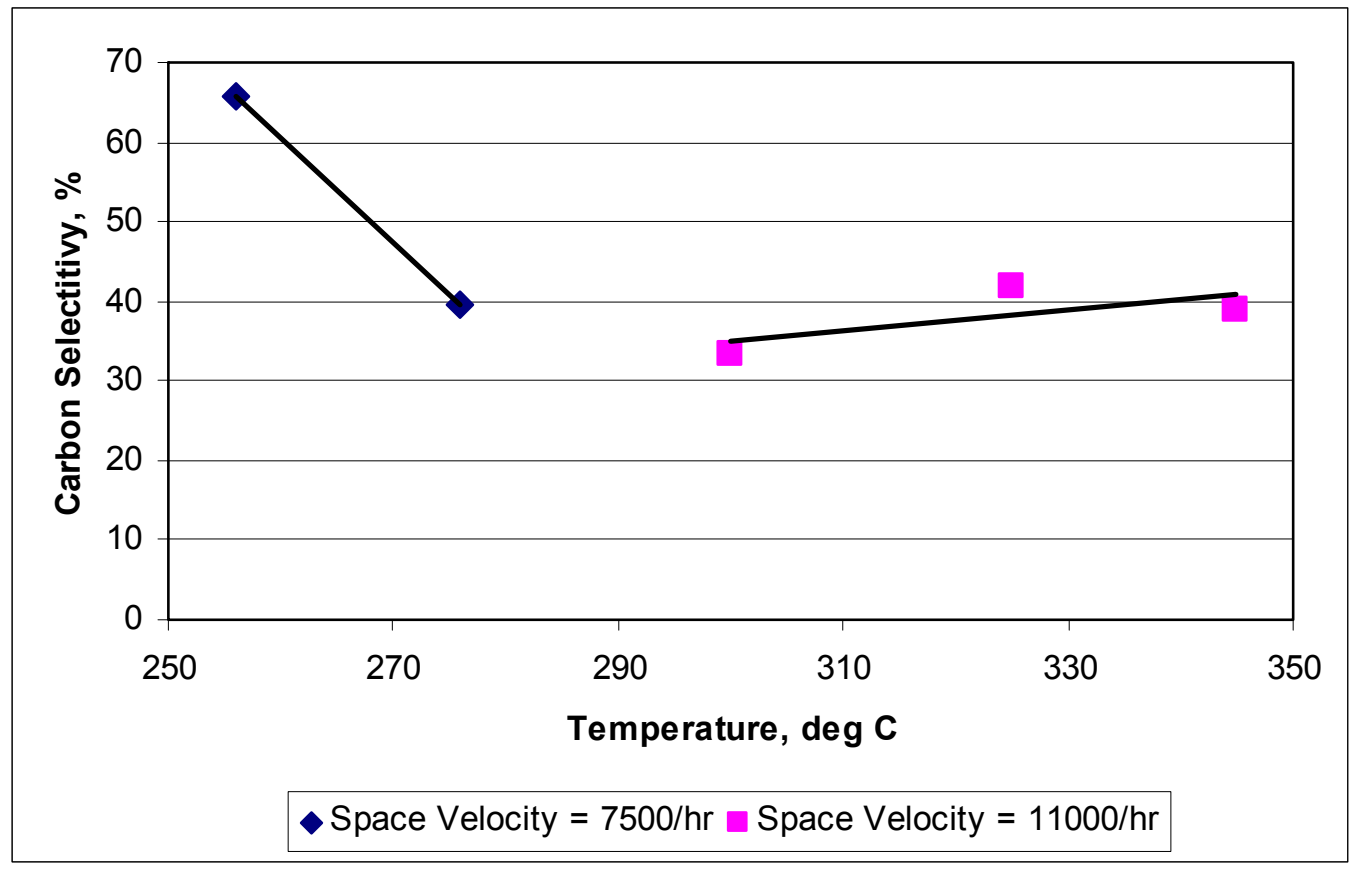

Figure B.66. Converted Carbon Selectivity to $\mathrm{C}_{2}+$ Oxygenates for the In-Promoted Catalyst

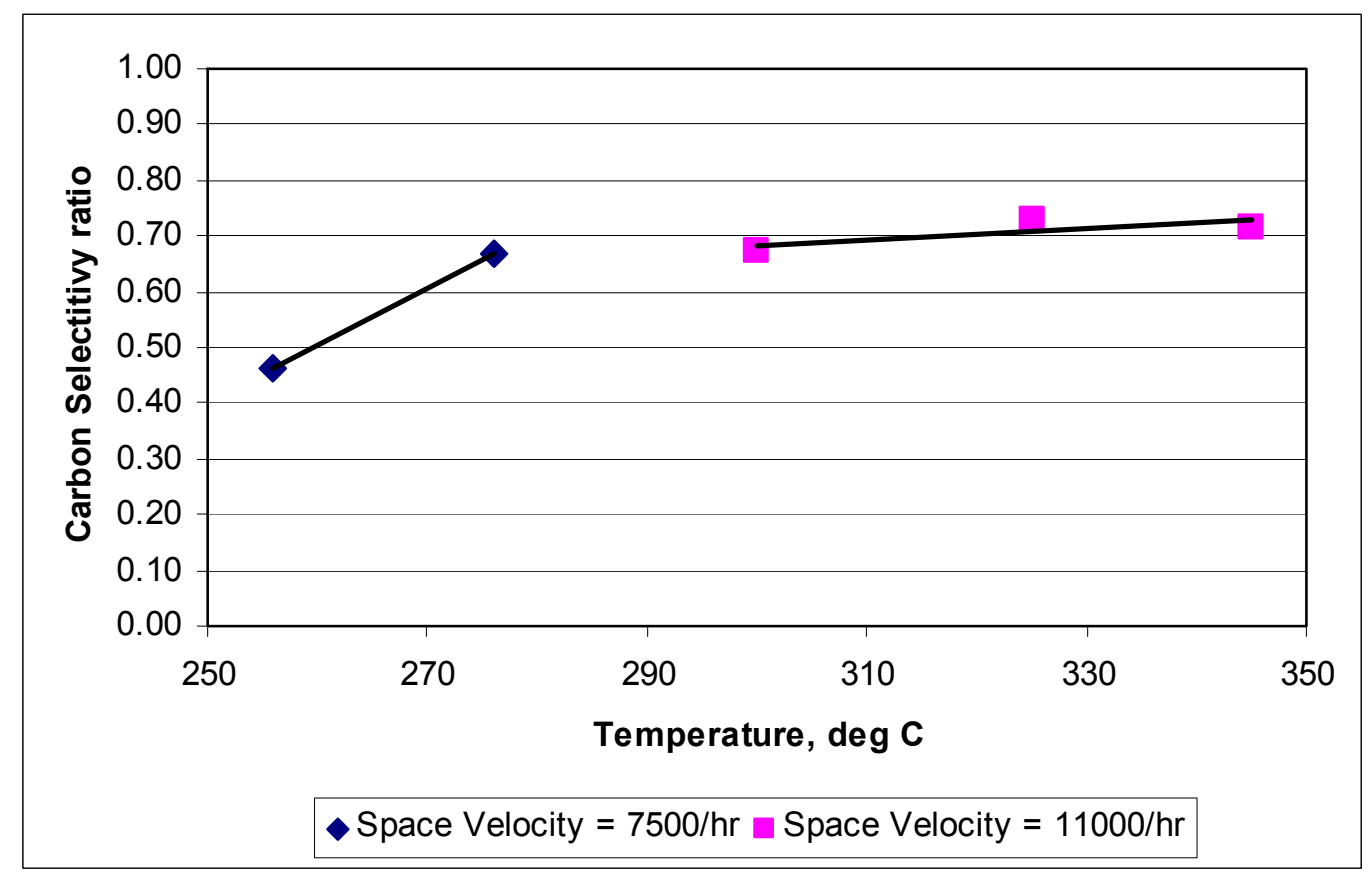

Figure B.67. Carbon Selectivity of All Oxygenates to $\mathrm{C}_{2}+$ Alcohols for the In-Promoted Catalyst 


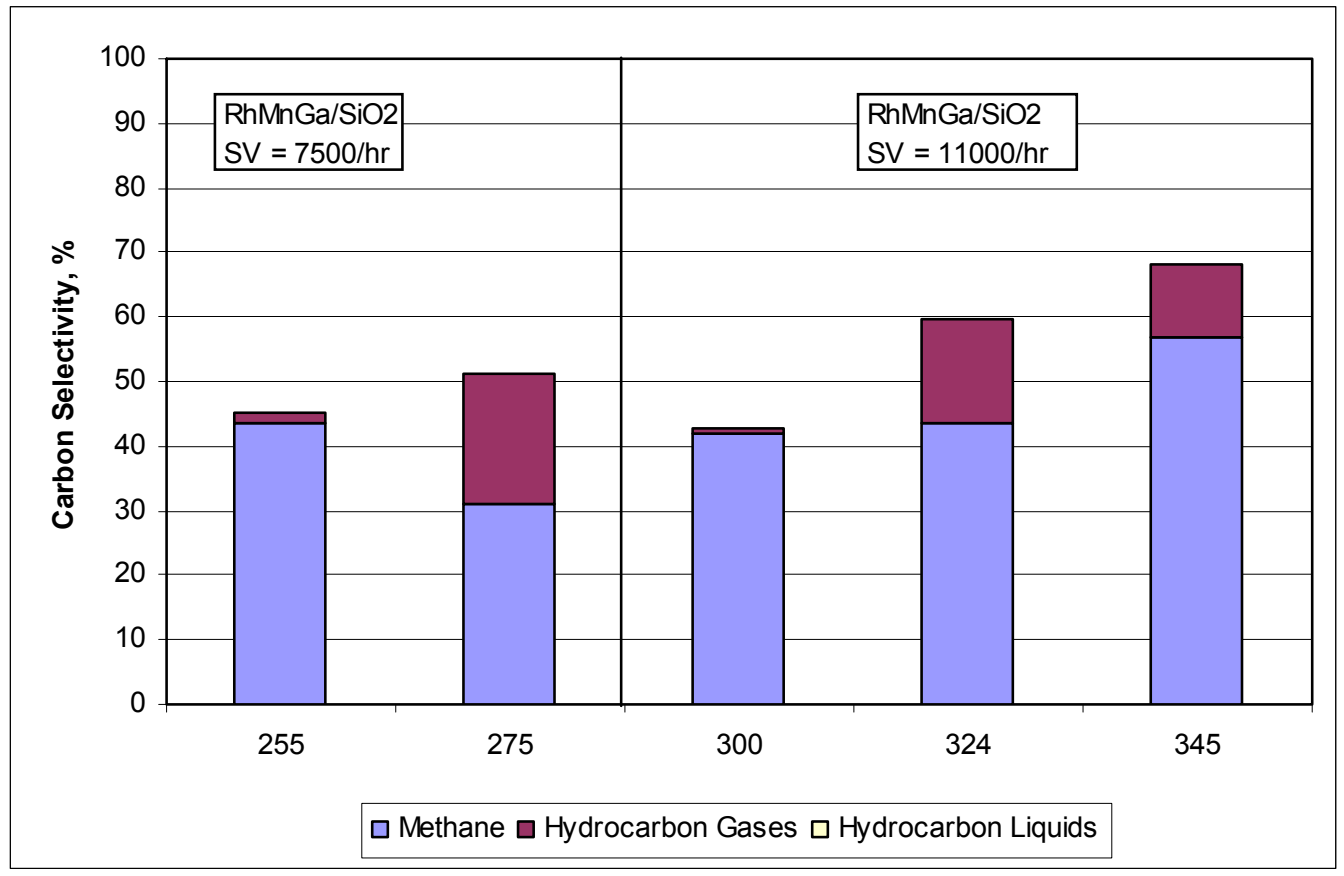

Figure B.68. Comparison of Hydrocarbon Selectivity for the In-Promoted Catalyst

\section{B.14.1 Sn-Promoted Catalyst}

The Sn-promoted catalyst $\left(\mathrm{RhMnSn} / \mathrm{SiO}_{2}\right)$ catalyst was reduced at the maximum temperature of $350^{\circ} \mathrm{C}$ and tested using the hot oil circulating system to heat the reactor. The catalyst testing sequence was 256 and $276^{\circ} \mathrm{C}$ at $7500 \mathrm{~L} / \mathrm{L}_{\text {cat }} / \mathrm{hr} ; 299,314$, and $325^{\circ} \mathrm{C}$ at $11,000 \mathrm{~L} / \mathrm{L}_{\text {cat }} / \mathrm{hr} ; 325^{\circ} \mathrm{C}$ at $15,000 \mathrm{~L} / \mathrm{L}_{\text {cat }} / \mathrm{hr}$; and $345^{\circ} \mathrm{C}$ at $11,000 \mathrm{~L} / \mathrm{L}_{\mathrm{cat}} / \mathrm{hr}$. The temperature control was very good for the first five test conditions with the catalyst varying $\pm 1.5^{\circ} \mathrm{C}$ for all test conditions. Two $7.5^{\circ} \mathrm{C}$ temperature excursions that lasted 15 minutes occurred during sample collection for the test condition at $325^{\circ} \mathrm{C}$ and $11,000 \mathrm{~L} / \mathrm{L}_{\mathrm{cat}} / \mathrm{hr}$, and five temperature excursions of approximately $20^{\circ} \mathrm{C}$ lasting approximately 30 minutes each were spaced between 6 and 7 hours apart during sample collection for the test condition at $342^{\circ} \mathrm{C}$ and $11,000 \mathrm{~L} / \mathrm{L}_{\text {cat }} / \mathrm{hr}$.

Figure B.69 shows that carbon conversion increased with increasing temperature as expected, but the effect of the space velocity was less clear. It appears that carbon conversion was unaffected when the space velocity was increased from 7500 to $11,000 \mathrm{~L} / \mathrm{L}_{\text {cat }} / \mathrm{hr}$, but was decreased when the space velocity was further increased to $15,000 \mathrm{~L} / \mathrm{L}_{\text {cat }} / \mathrm{hr}$. There was a significant increase in the $\mathrm{C}_{2}+$-oxygenate STYs between 276 and $299^{\circ} \mathrm{C}$ as shown in Figure B.70. This increase is attributed to a corresponding increase in the carbon selectivity to $\mathrm{C}_{2}+$ oxygenates between these temperatures as shown in Figure B.71. The maximum $\mathrm{C}_{2}+$-oxygenate STY of $355 \mathrm{~g} / \mathrm{L}_{\text {cat }} / \mathrm{hr}$ occurred at $325^{\circ} \mathrm{C}$ and at a $11,000 \mathrm{~L} / \mathrm{L}_{\text {cat }} / \mathrm{hr}$ space velocity. According to Figure B.72, the selectivity of all oxygenates to $\mathrm{C}_{2}+$ alcohols increased with increasing temperature to a maximum carbon selectivity of $53 \%$ at $345^{\circ} \mathrm{C}$. The Sn-promoted catalyst produced a significant amount of hydrocarbon liquids at all but the first test condition, as shown in Figure B.73, although it appears that there was a significant reduction in the amount produced between 276 and $299^{\circ} \mathrm{C}$. At higher temperatures, the selectivity towards hydrocarbon liquids and higher hydrocarbon gases remained relatively constant, suggesting very little further deactivation of the hydrocarbon chain-growth rate. 


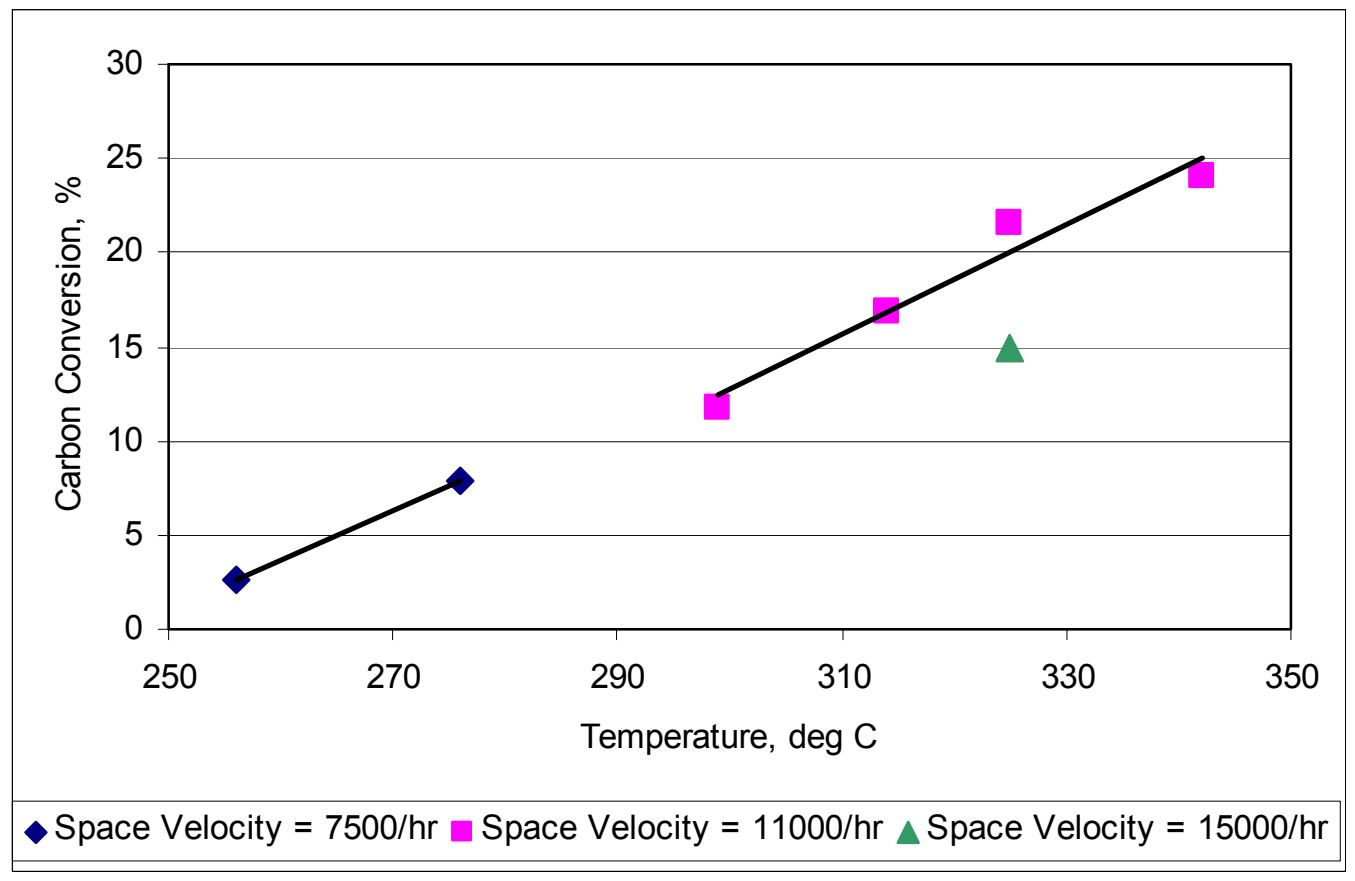

Figure B.69. Carbon Conversion for the Sn-Promoted Catalyst

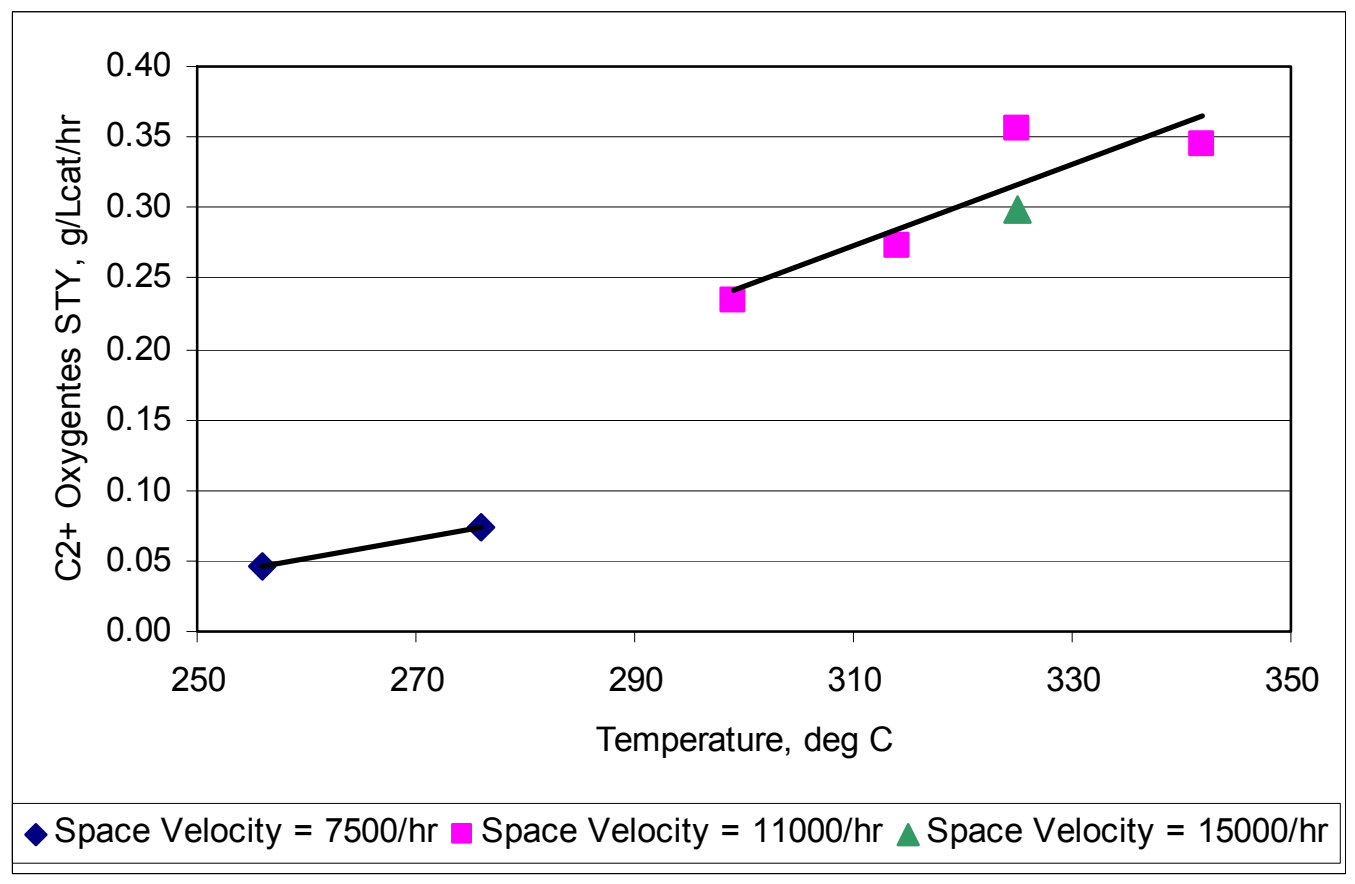

Figure B.70. $\mathrm{C}_{2}+-$ Oxygenate STYs for the Sn-Promoted Catalyst 


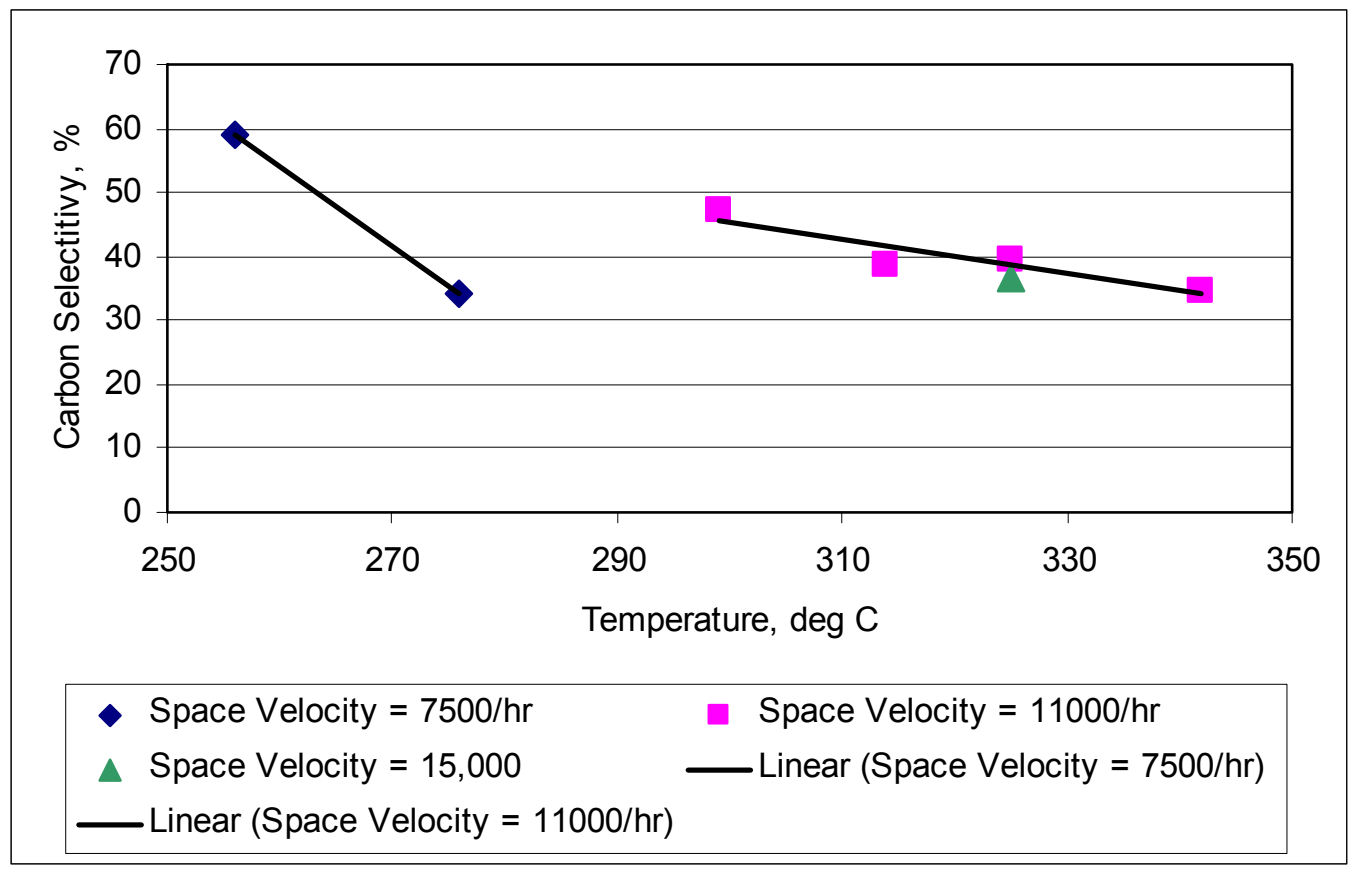

Figure B.71. Converted Carbon Selectivity to $\mathrm{C}_{2}+$ Oxygenates for the Sn-Promoted Catalyst

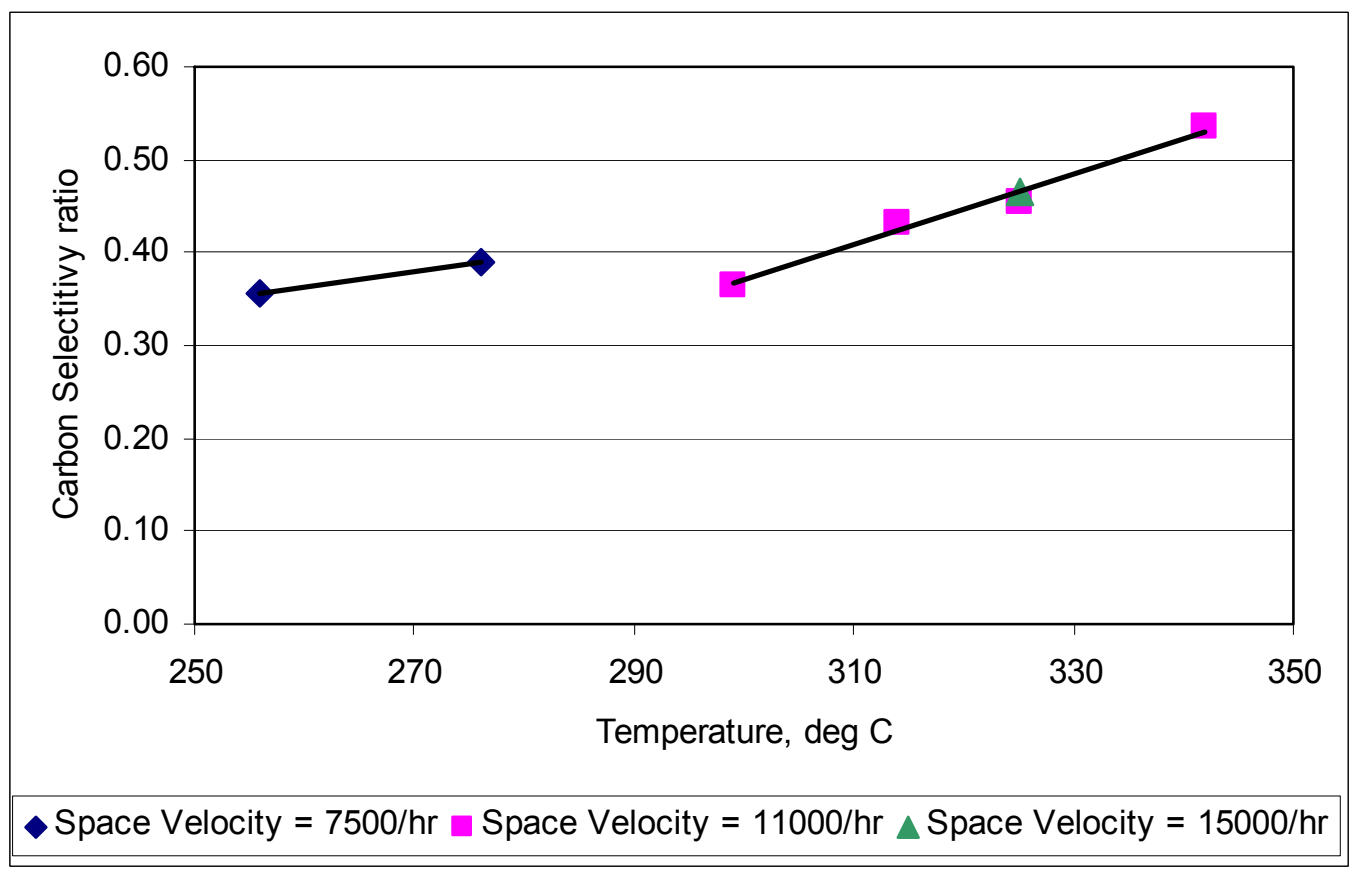

Figure B.72. Carbon Selectivity of All Oxygenates to $\mathrm{C}_{2}+$ Alcohols for the Sn-Promoted Catalyst 


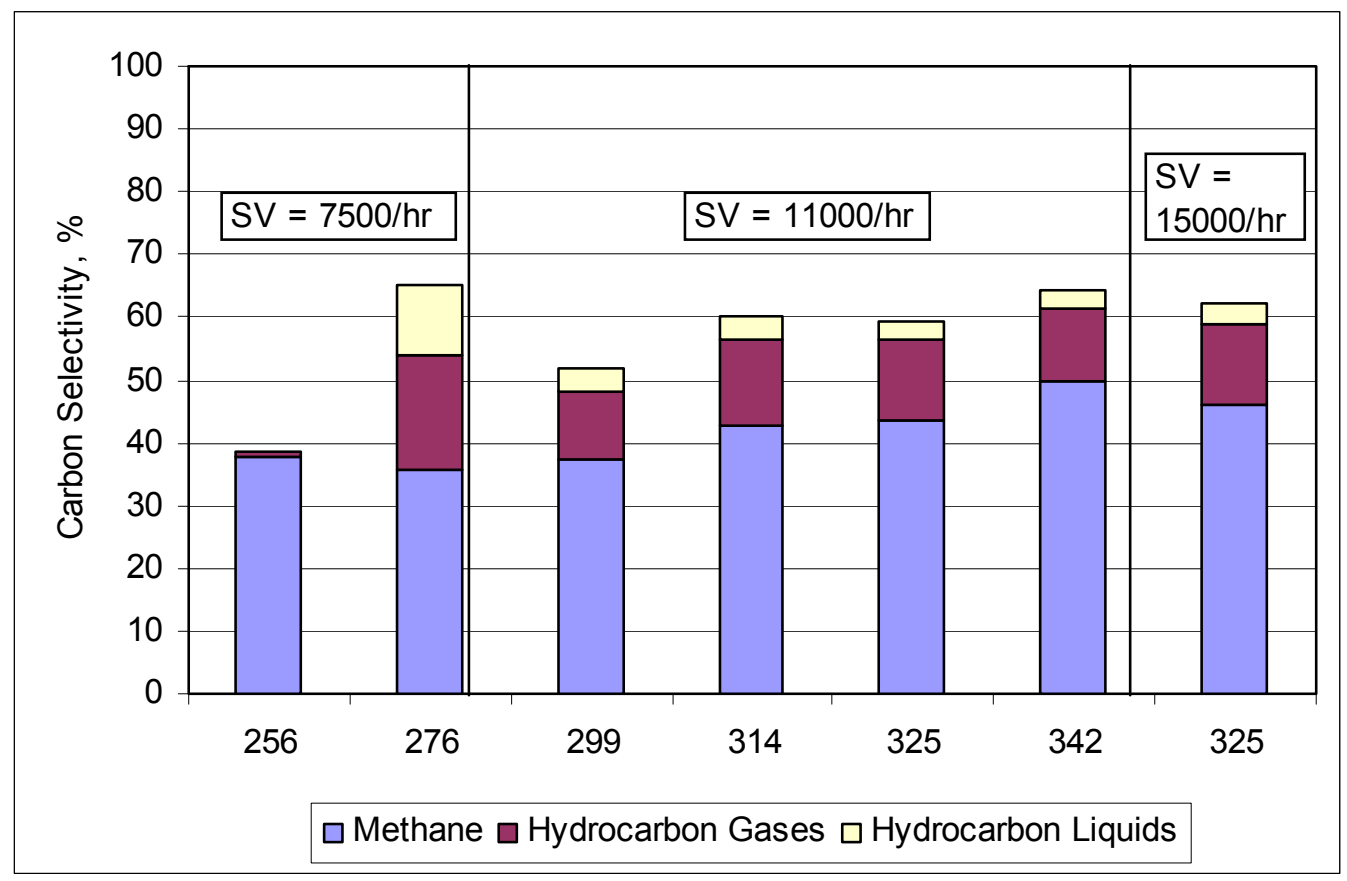

Figure B.73. Comparison of Hydrocarbon Selectivity for the Sn-Promoted Catalyst

\section{B.15 Au-Promoted Catalyst}

The Au-promoted catalyst $\left(\mathrm{RhMnAu} / \mathrm{SiO}_{2}\right)$ catalyst was reduced at the maximum temperature of $350^{\circ} \mathrm{C}$ and tested using the hot oil circulating system to heat the reactor. The catalyst testing sequence was 257 and $276^{\circ} \mathrm{C}$ at 7500 at L/L $/ \mathrm{L}_{\text {cat }} / \mathrm{hr} ; 300$ and $316^{\circ} \mathrm{C}$ at $11,000 \mathrm{~L} / \mathrm{L}_{\text {cat }} / \mathrm{hr} ; 314^{\circ} \mathrm{C}$ at $15,000 \mathrm{~L} / \mathrm{L}_{\text {cat }} / \mathrm{hr}$; $276^{\circ} \mathrm{C}$ at $7500 \mathrm{~L} / \mathrm{L}_{\text {cat }} / \mathrm{hr} ; 327^{\circ} \mathrm{C}$ at $15,000 \mathrm{~L} / \mathrm{L}_{\text {cat }} / \mathrm{hr}$; and $325^{\circ} \mathrm{C}$ at $19,000 \mathrm{~L} / \mathrm{L}_{\text {cat }} / \mathrm{hr}$. The temperature control was very good for the all test conditions, except the third test condition, with the catalyst varying $\pm 1.0^{\circ} \mathrm{C}$. However, when the catalyst temperature was increased to $318^{\circ} \mathrm{C}$ at a $11,000 \mathrm{~L} / \mathrm{L}_{\text {cat }} / \mathrm{hr}$ space velocity, for the third test condition, it maintained that temperature for approximately 10 hours and then underwent a rapid $18^{\circ} \mathrm{C}$ temperature decrease followed by a $15^{\circ} \mathrm{C}$ temperature rise to $315^{\circ} \mathrm{C}$ where it remained for the remaining 11 hours of the liquid sample collection period. The average temperature for the run was reported as $316^{\circ} \mathrm{C}$.

Figure B.74 shows the carbon conversion for the various conditions using the Au-promoted catalyst. There is a general trend of increasing carbon conversion with temperature and decreasing carbon conversion with space velocity. However, above $310^{\circ} \mathrm{C}$, there also appears to be a deactivation of the catalyst, which is evidenced by the decreasing carbon conversion with temperature for the two conditions at $15,000 \mathrm{~L} / \mathrm{L}_{\text {cat }} /$ hr space velocity. Figure B.75, which shows the $\mathrm{C}_{2}+$-oxygenate $\mathrm{STY}$ s for the various conditions, also indicates a loss of activity for conditions above $310^{\circ} \mathrm{C}$. Increasing the temperature from $300^{\circ} \mathrm{C}$ to $316^{\circ} \mathrm{C}$ resulted in a decrease in the STY. However, this could have been attributed to a relatively high carbon conversion of approximately $33 \%$ at $316^{\circ} \mathrm{C}$. Increasing the space velocity to $15,000 \mathrm{~L} / \mathrm{L}_{\text {cat }} / \mathrm{hr}$ at the same temperature did improve the STY, while reducing carbon conversion to approximately $24 \%$, but subsequent increase in the temperature to $327^{\circ} \mathrm{C}$ at the higher space velocity resulted in a significant decrease in the STY, while carbon conversion remained about the same. 
Increasing the space velocity again to $19,000 \mathrm{~L} / \mathrm{L}_{\text {cat }} / \mathrm{hr}$ at about the same temperature $\left(325^{\circ} \mathrm{C}\right)$ improved the $\mathrm{C}_{2}+$-oxygenate $\mathrm{STY}$, even though the carbon conversion was relatively low (approximately 20\%). The repeat of conditions at $276^{\circ} \mathrm{C}$ and $11,000 \mathrm{~L} / \mathrm{L}_{\text {cat }} / \mathrm{hr}$ produced a lower carbon conversion and $\mathrm{C}_{2}+$-oxygenate STY, confirming some degree of catalyst deactivation.

According to Figure B.76 the carbon selectivity to $\mathrm{C}_{2}+$ oxygenates showed a consistent improvement with increasing space velocity, with the effect most pronounced between $7500 \mathrm{~L} / \mathrm{L}_{\text {cat }} / \mathrm{hr}$ and $11,000 \mathrm{~L} / \mathrm{L}_{\text {cat }} / \mathrm{hr}$. The effect for the higher space velocities was much smaller. Carbon selectivity to $\mathrm{C}_{2}+$ oxygenates decreased with increasing temperature for a specified space velocity, as expected. In general the selectivity of all oxygenates to $\mathrm{C}_{2}+$ alcohols increased with increasing temperature as expected for all test conditions except the repeat condition as shown in Figure B.77. The latter condition is an anomaly that is not easily explained at this time. The repeat condition also had a $14 \%$ selectivity of all oxygenates to methanol, compared to the next highest selectivity to methanol of $2.5 \%$ observed for the last condition tested $\left(325^{\circ} \mathrm{C}\right.$ at $\left.19,000 \mathrm{~L} / \mathrm{L}_{\text {cat }} / \mathrm{hr}\right)$. Examination of Figure B.78 also shows that hydrocarbon liquids were not produced for the last three conditions tested including the repeat condition. The beneficial effect of improved $\mathrm{C}_{2}+$-oxygenate selectivity with higher space velocity when the carbon conversion was below $30 \%$ had not been previously observed, and may suggest a different mechanism relating hydrocarbon and oxygenate selectivity.

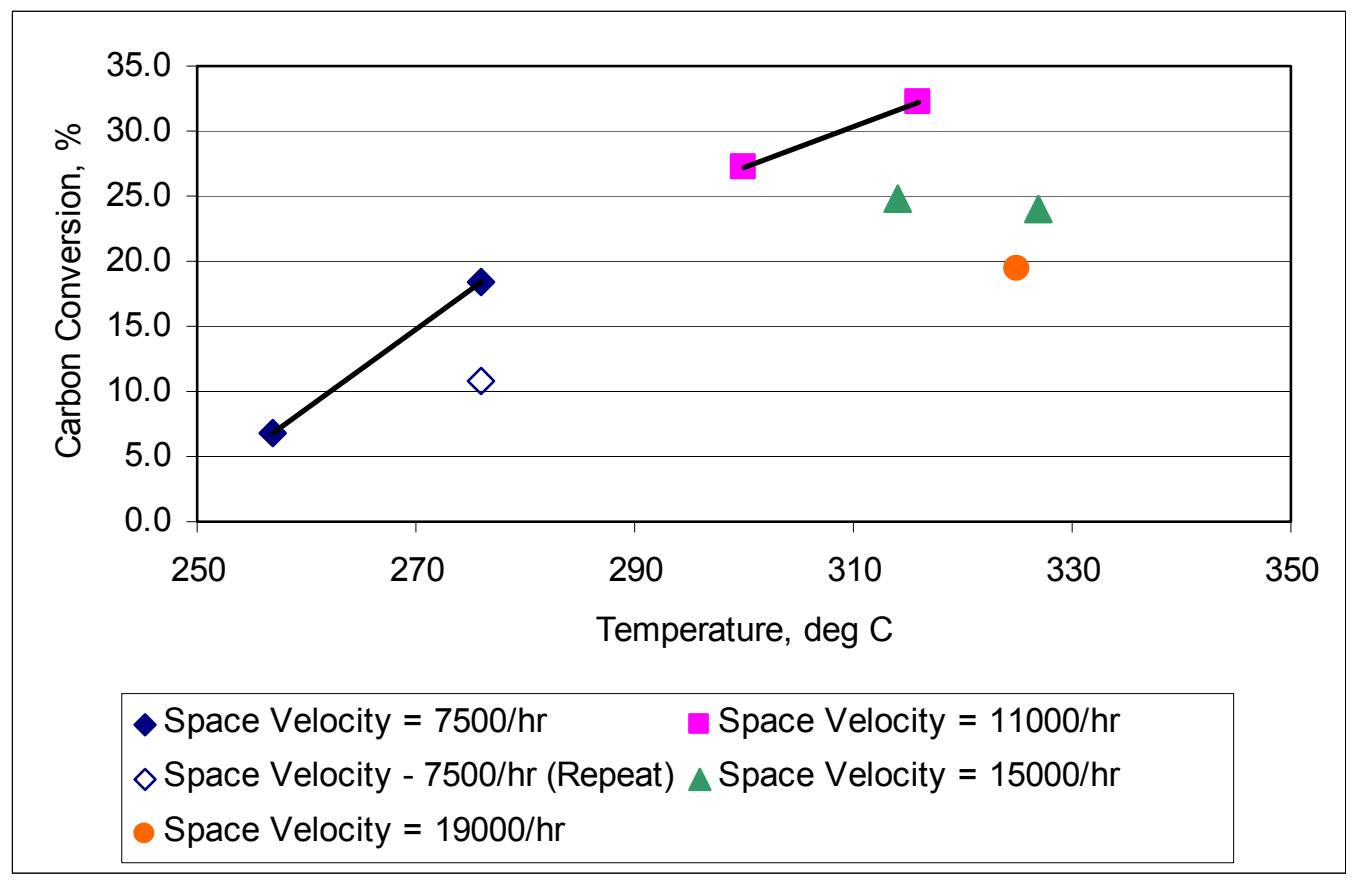

Figure B.74. Carbon Conversion for the Au-Promoted Catalyst 


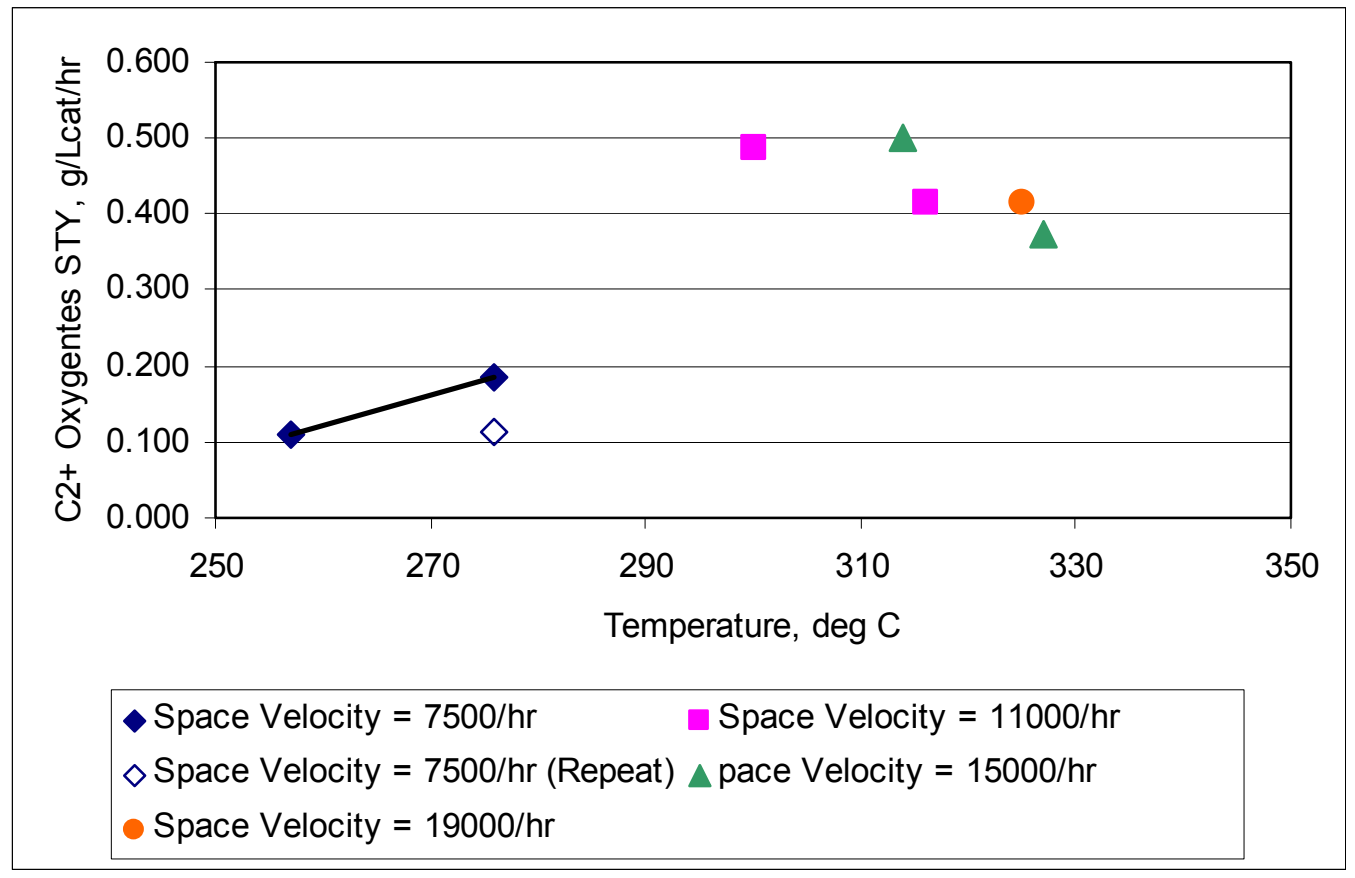

Figure B.75. $\mathrm{C}_{2}+-$ Oxygenate STYs for the Au-Promoted Catalyst

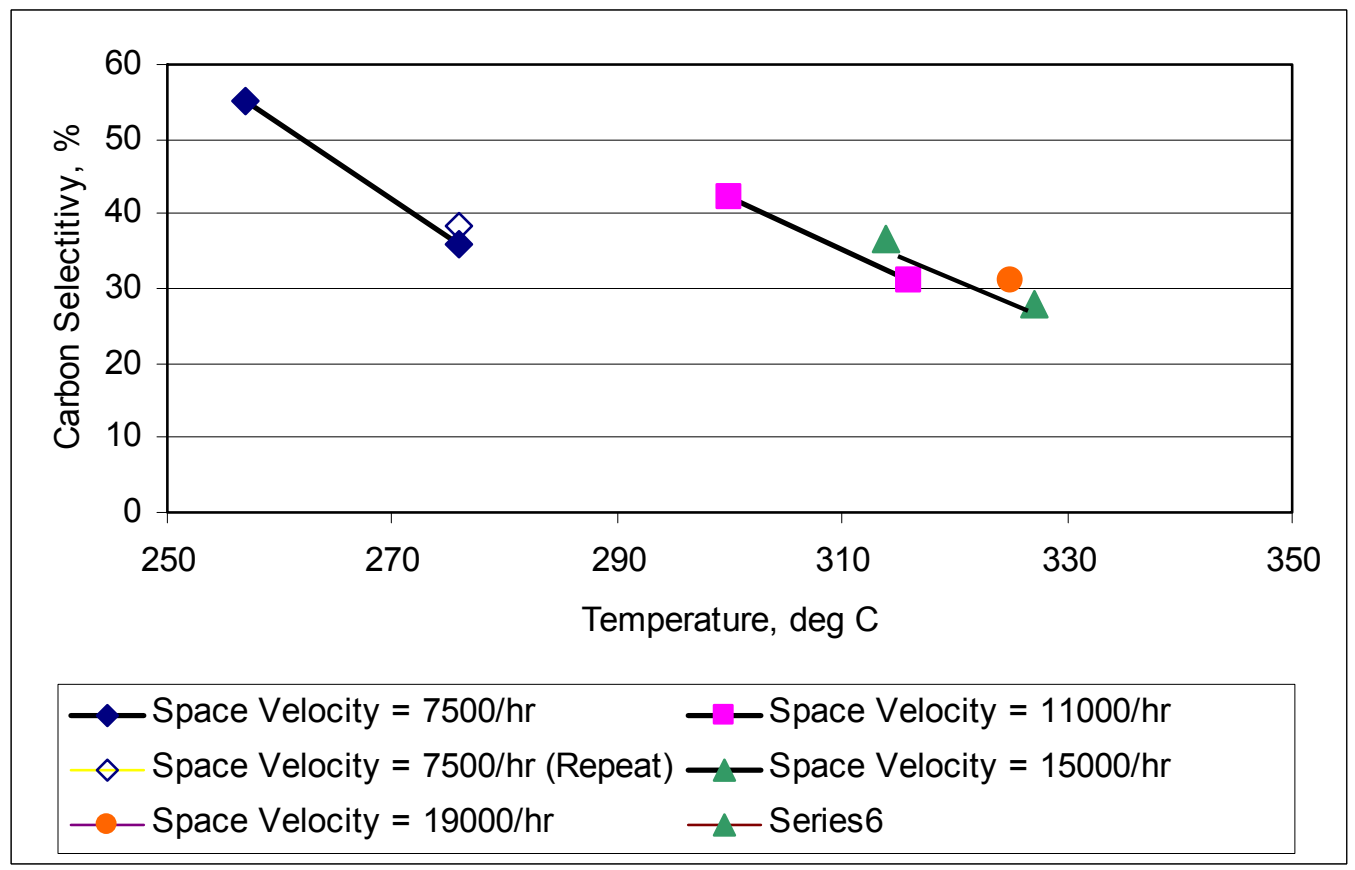

Figure B.76. Converted Carbon Selectivity to $\mathrm{C}_{2}+$ Oxygenates for the Au-Promoted Catalyst 


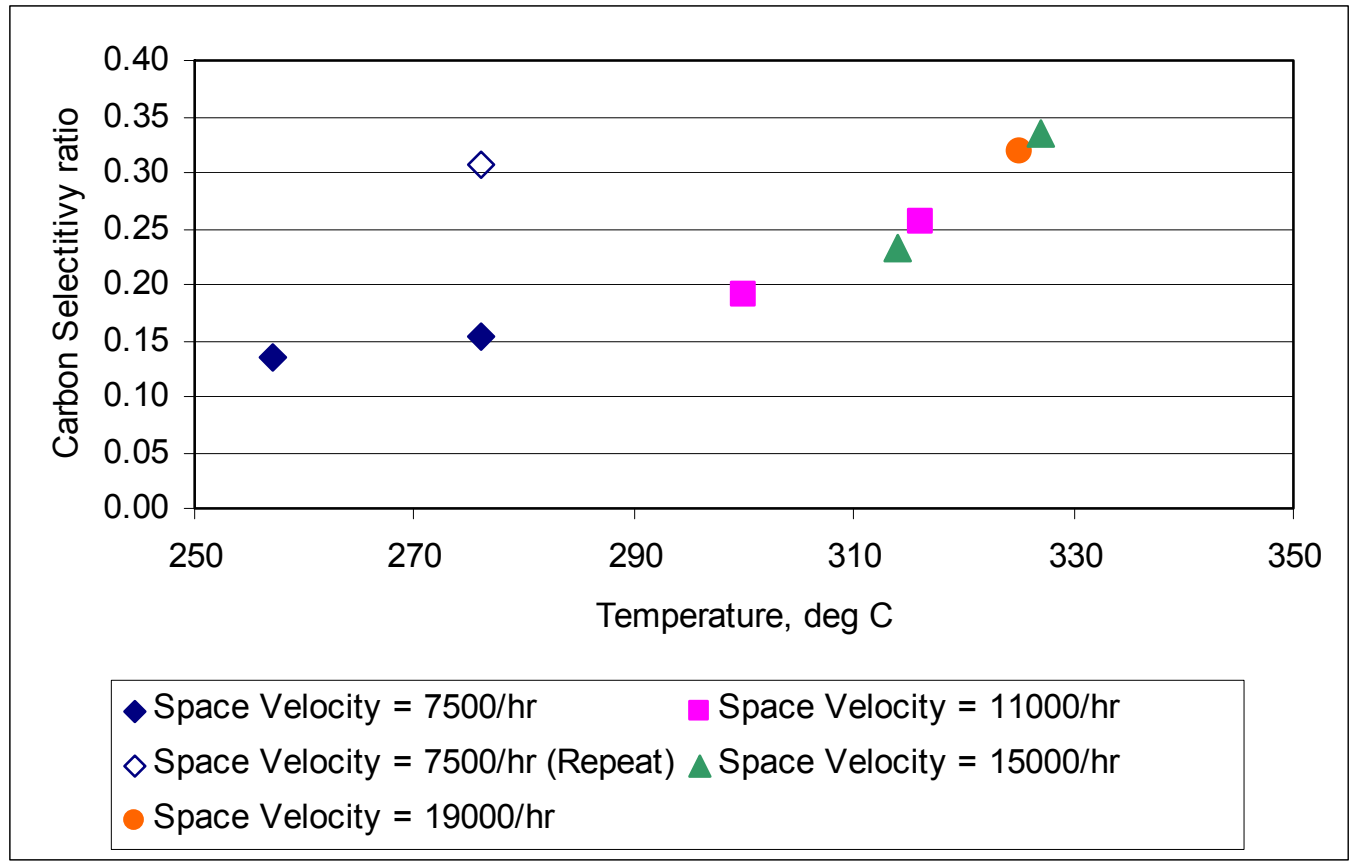

Figure B.77. Carbon Selectivity of All Oxygenates to $\mathrm{C}_{2}+$ Alcohols for the Au-Promoted Catalyst

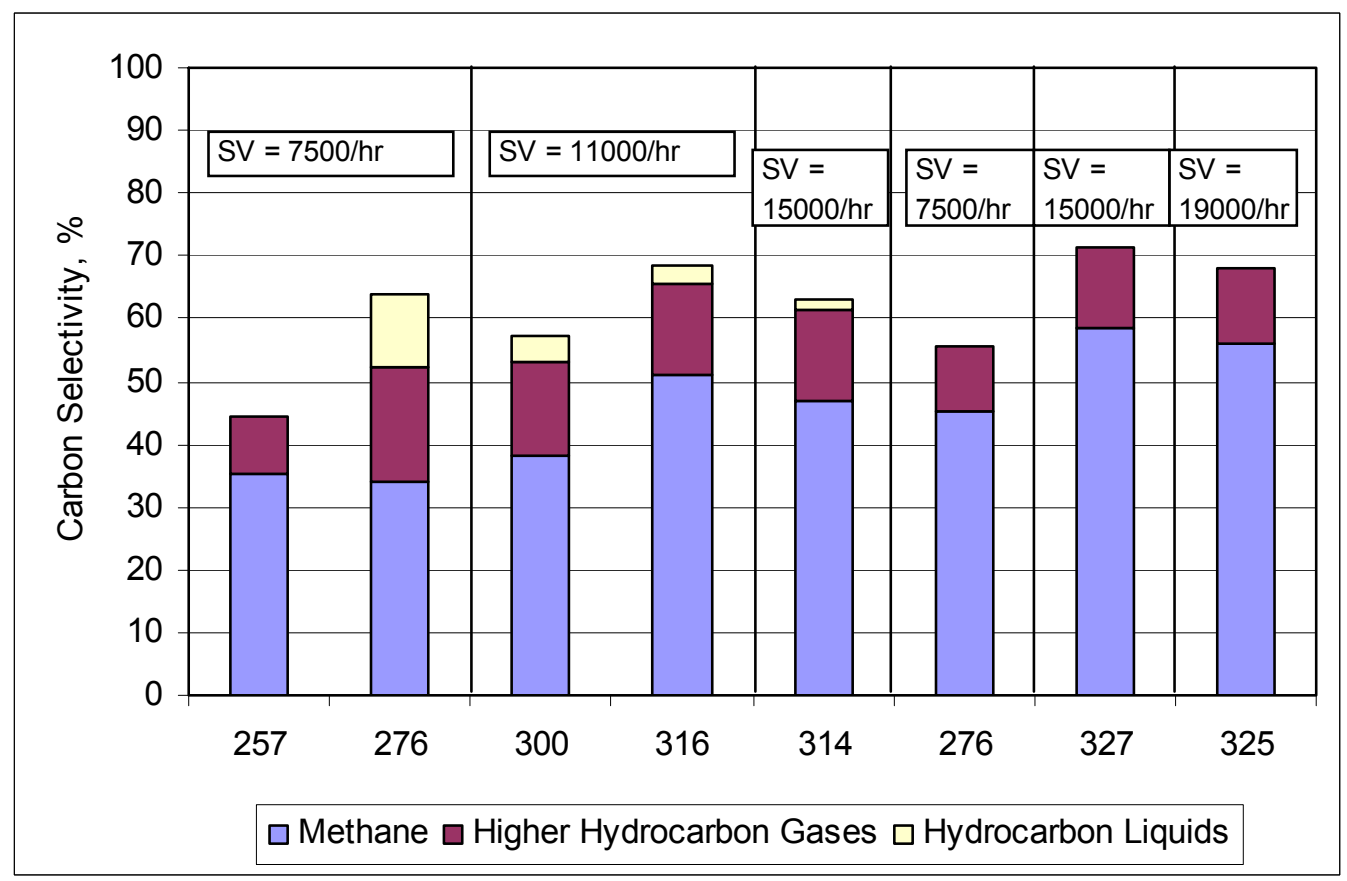

Figure B.78. Comparison of Hydrocarbon Selectivity for the Au-Promoted Catalyst 


\section{B.16 Ag-Promoted Catalyst}

The Ag-promoted catalyst $(\mathrm{RhMnAg} / \mathrm{SiO} 2)$ catalyst was reduced at the maximum temperature of $350^{\circ} \mathrm{C}$ and tested using the hot oil circulating system to heat the reactor. The catalyst testing sequence was 256 and $276^{\circ} \mathrm{C}$ at 7500 at $\mathrm{L} / \mathrm{L}_{\mathrm{cat}} / \mathrm{hr}$, and at 300 and $325^{\circ} \mathrm{C}$ at $11,000 \mathrm{~L} / \mathrm{L}_{\mathrm{cat}} / \mathrm{hr}$. The temperature control was very good for all test conditions with the catalyst temperatures varying $\pm 1.0^{\circ} \mathrm{C}$ about the reported values.

Carbon conversion for the Ag-promoted catalyst was relatively low ranging from $2 \%$ at $256^{\circ} \mathrm{C}$ and $7500 \mathrm{~g} / \mathrm{L}_{\text {cat }} / \mathrm{hr}$ to $12 \%$ at $300^{\circ} \mathrm{C}$ at $11,000 \mathrm{~g} / \mathrm{L}_{\text {cat }} / \mathrm{hr}$, as shown in Figure B.79. The $\mathrm{C}_{2}+$-oxygenate STYs were also relatively low, reaching a maximum of $106 \mathrm{~g} / \mathrm{L}_{\text {cat }} / \mathrm{hr}$ as shown in Figure B.80. Figure B.81 shows that the carbon selectivity decreased with increasing temperature in a regular fashion that appeared to be independent of the space velocity, reaching a low of approximately $21 \%$ at the maximum temperature tested. The carbon selectivity of all oxygenates to $\mathrm{C}_{2}+$ alcohols increased with increasing temperature as might be expected, with a maximum selectivity of $51 \%$ at $325^{\circ} \mathrm{C}$. The Ag-promoted catalyst also produced significant quantities of methanol, with the selectivity of all oxygenates to methanol ranging from 17 to $22 \%$. Figure B.83 shows that the catalyst did not produce hydrocarbon liquids at any of the conditions tested and only produced a small quantity of higher hydrocarbon gases at $325^{\circ} \mathrm{C}$.

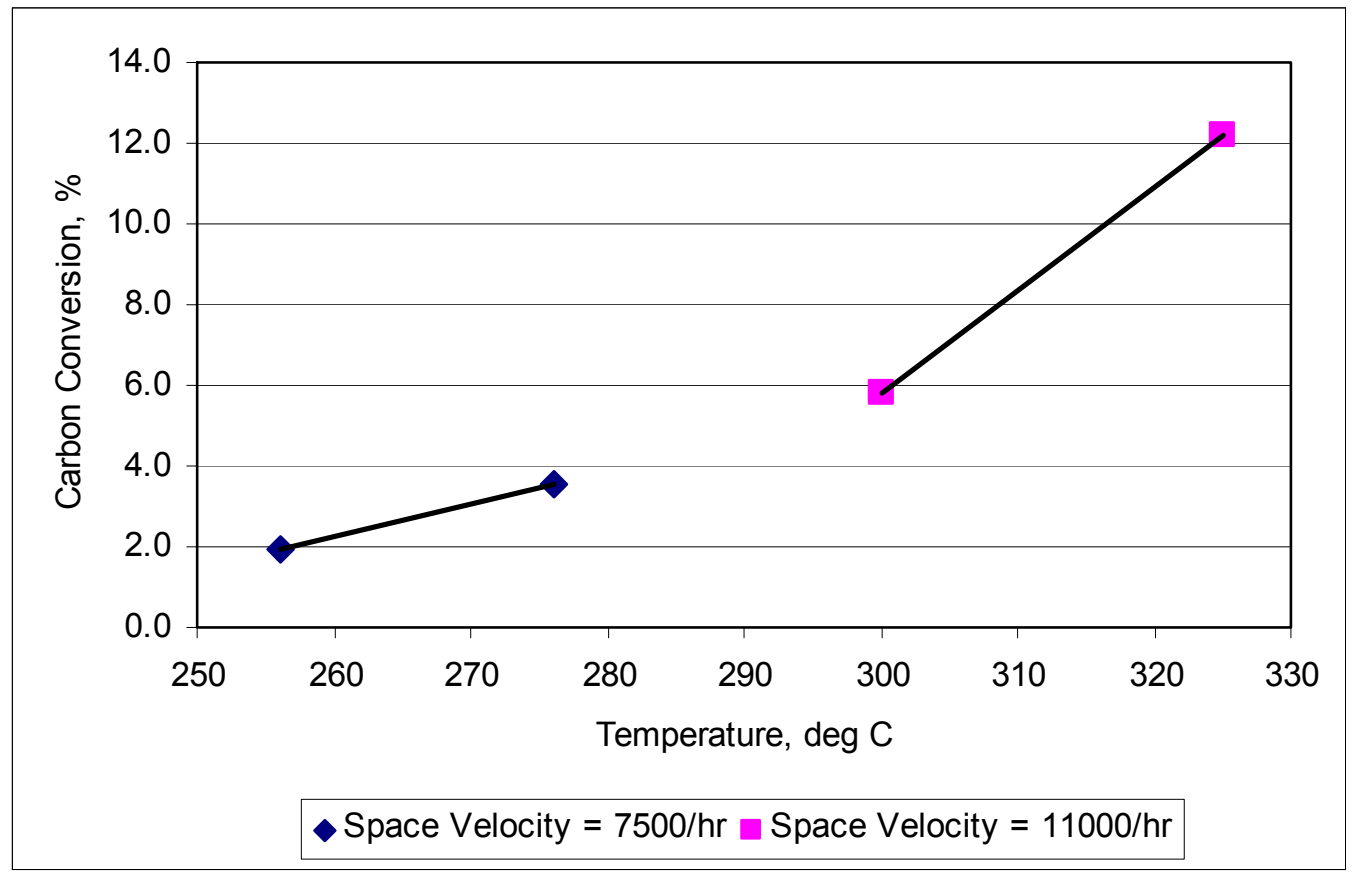

Figure B.79. Carbon Conversion for the Ag-Promoted Catalyst 


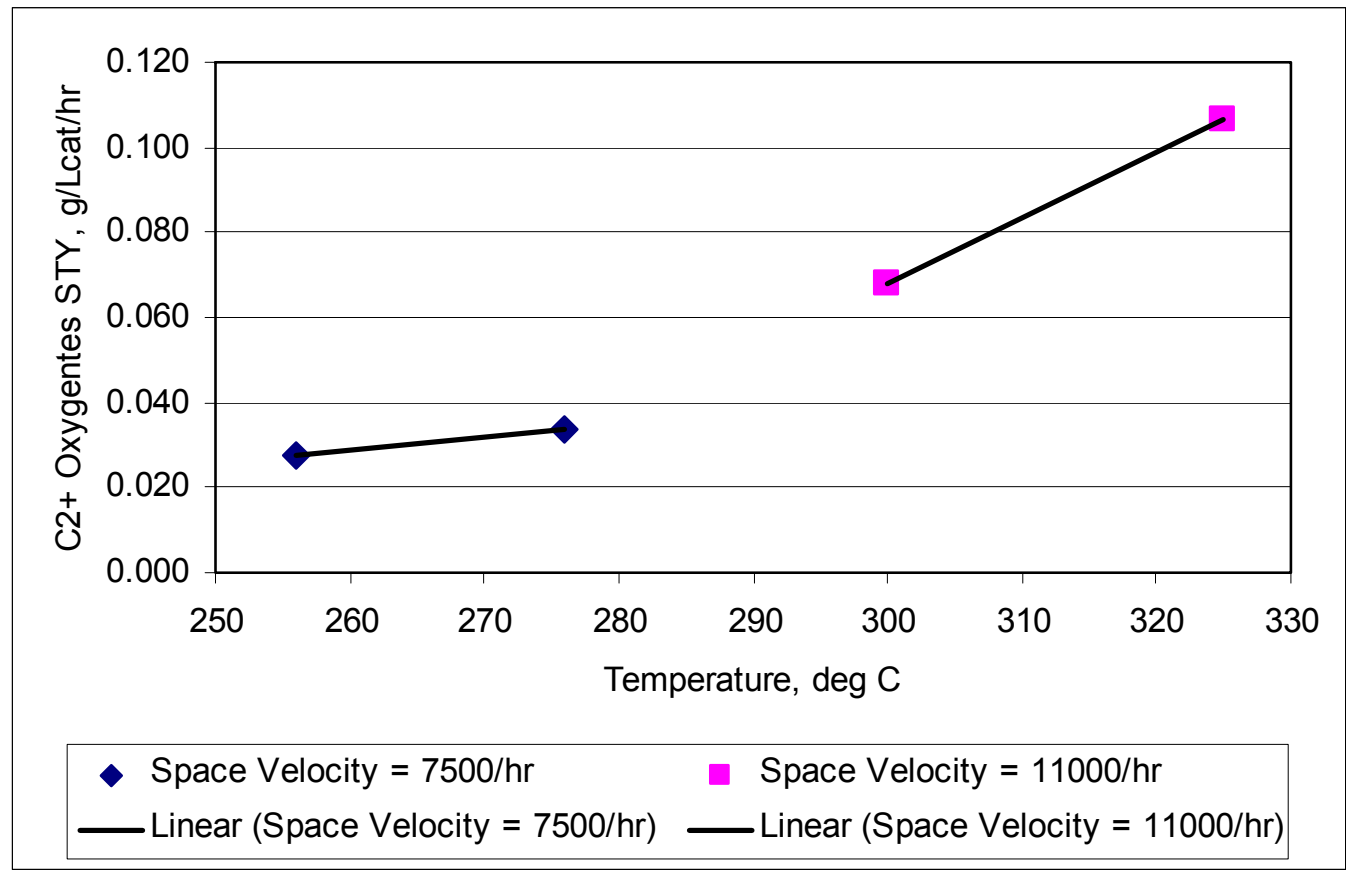

Figure B.80. $\mathrm{C}_{2}+-$ Oxygenate STYs for the Ag-Promoted Catalyst

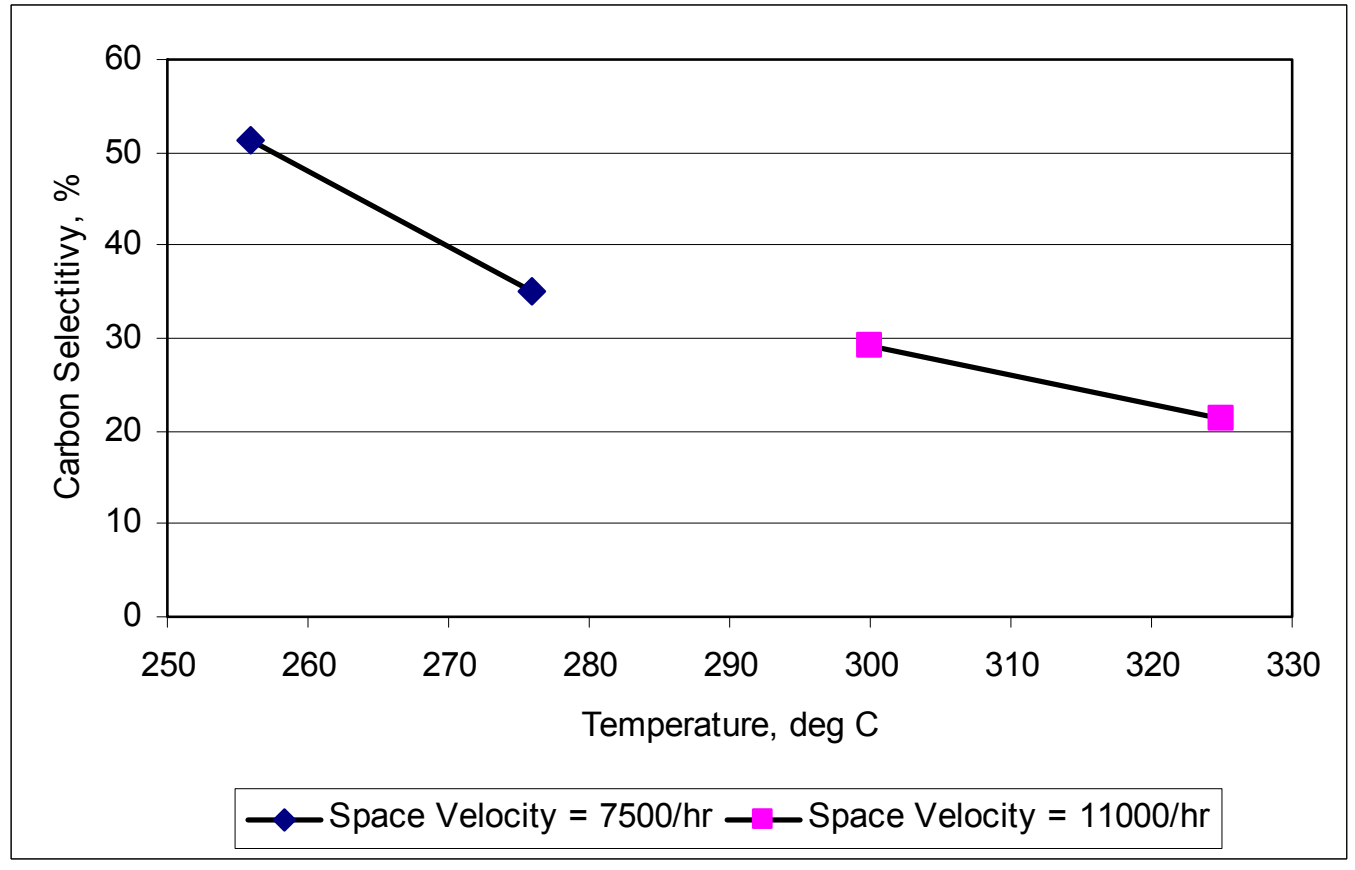

Figure B.81. Converted Carbon Selectivity to $\mathrm{C}_{2}+$ Oxygenates for the Ag-Promoted Catalyst 


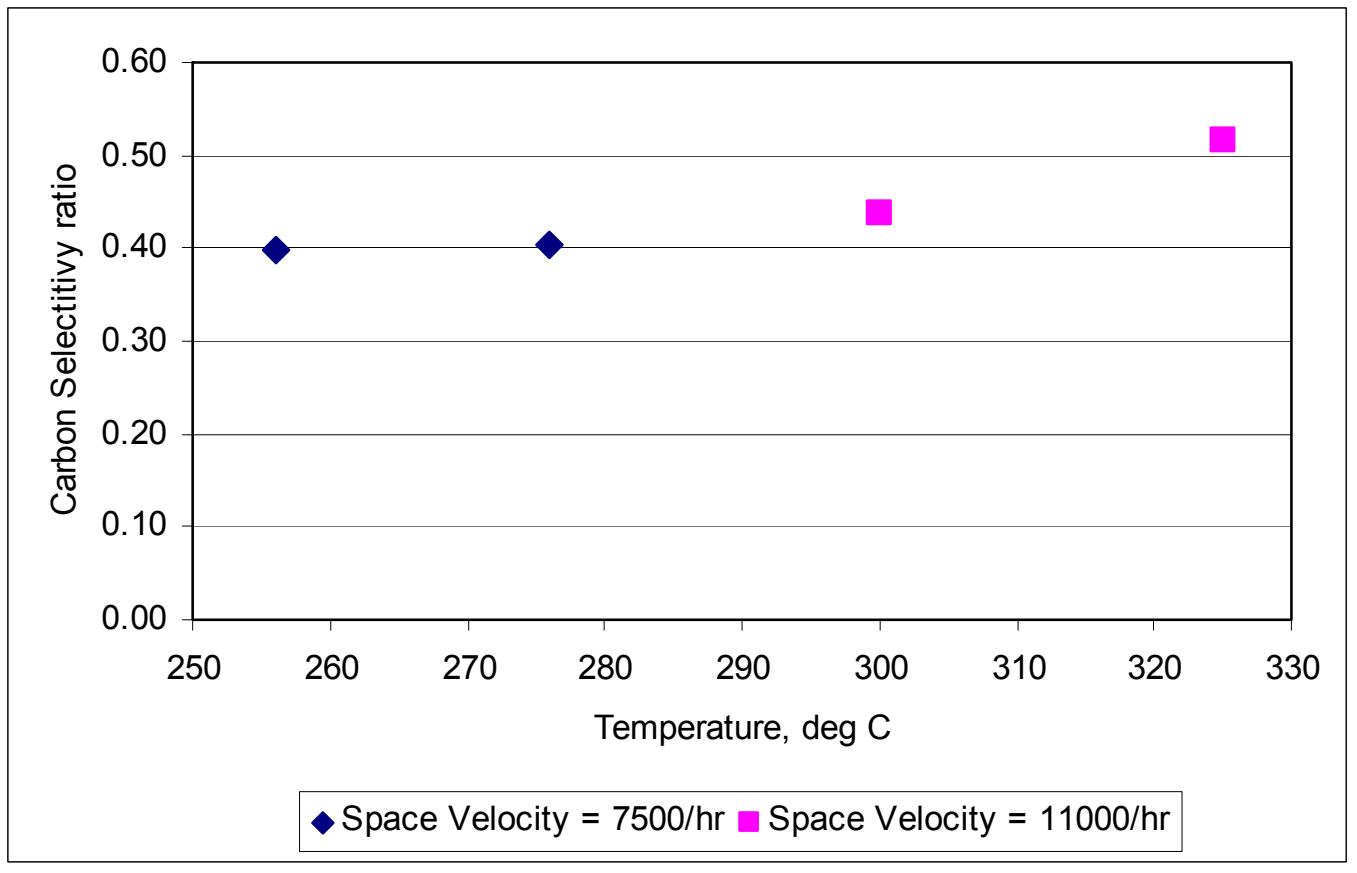

Figure B.82. Carbon Selectivity of All Oxygenates to $\mathrm{C}_{2}+$ Alcohols for the Ag-Promoted Catalyst

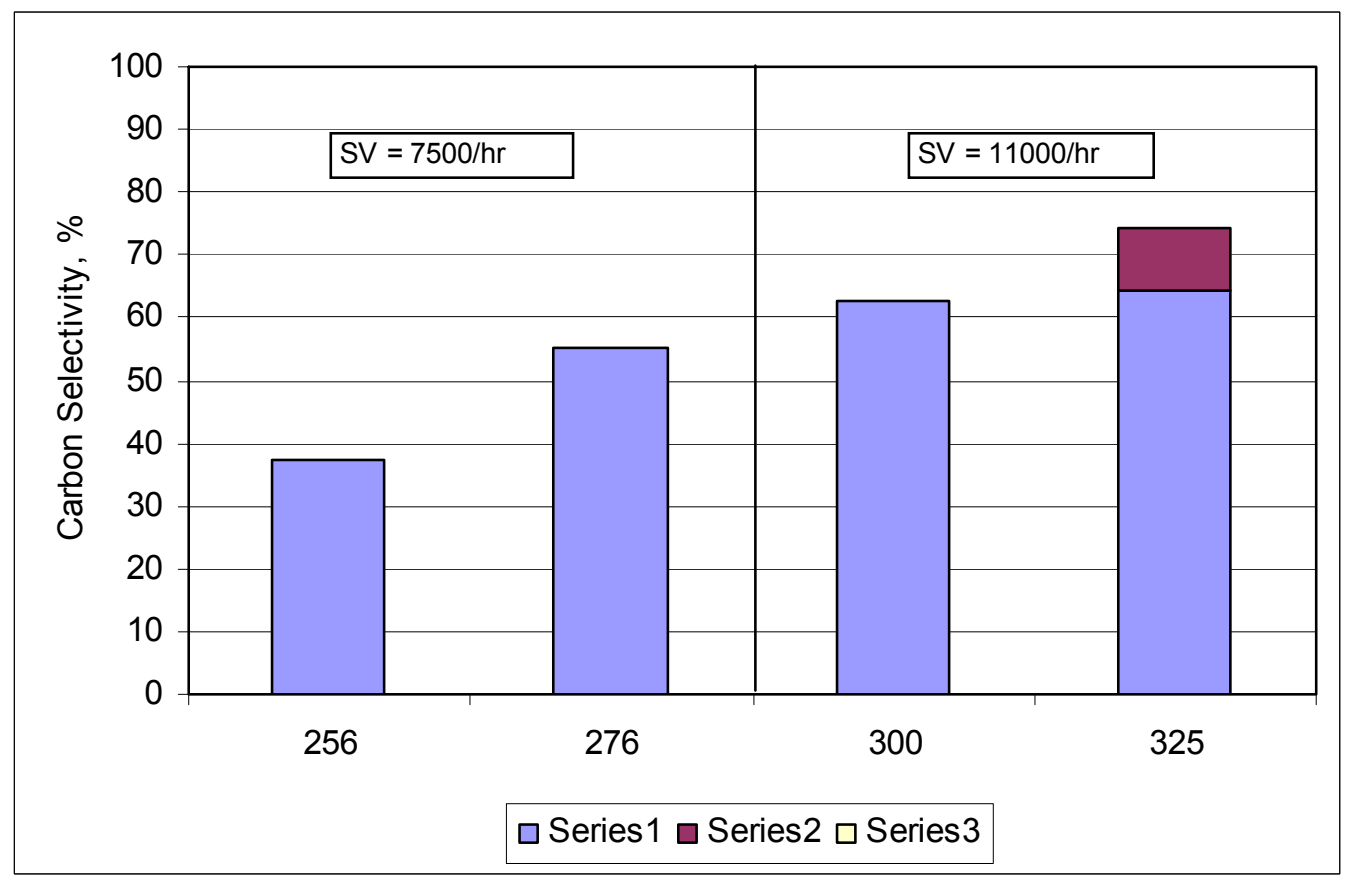

Figure B.83. Comparison of Hydrocarbon Selectivity for the Ag-Promoted Catalyst 


\section{B.17 V-Promoted Catalyst}

The V-promoted catalyst $\left(\mathrm{RhMnV} / \mathrm{SiO}_{2}\right)$ catalyst was reduced at a maximum temperature of $350^{\circ} \mathrm{C}$ and tested using the hot oil circulating system to heat the reactor. The catalyst testing sequence was 255 and $275^{\circ} \mathrm{C}$ at 7500 at $\mathrm{L} / \mathrm{L}_{\text {cat }} / \mathrm{hr}, 299$ and $314^{\circ} \mathrm{C}$ at $11,000 \mathrm{~L} / \mathrm{L}_{\text {cat }} / \mathrm{hr}, 315^{\circ} \mathrm{C}$ at $15,000 \mathrm{~L} / \mathrm{L}_{\text {cat }} / \mathrm{hr}$, and $275^{\circ} \mathrm{C}$ at $7500 \mathrm{~L} / \mathrm{L}_{\text {cat }} / \mathrm{hr}$. The temperature control was very good for all test conditions, except for the fourth test condition, with the catalyst varying $\pm 1.0^{\circ} \mathrm{C}$ for these test conditions. The temperature slowly decreased about $4^{\circ} \mathrm{C}$ during the sample collection period for the fourth condition for a reported average temperature of $314^{\circ} \mathrm{C} \pm 2^{\circ} \mathrm{C}$.

The V-promoted catalysts showed evidence of significant deactivation above $300^{\circ} \mathrm{C}$ as indicated in Figure B.84 by the decrease in carbon conversion between 299 and $314^{\circ} \mathrm{C}$ at $11,000 \mathrm{~L} / \mathrm{L}_{\mathrm{cat}} / \mathrm{hr}$, and with the repeat condition at $275^{\circ} \mathrm{C}$ and $7500 \mathrm{~L} / \mathrm{L}_{\text {cat }} / \mathrm{hr}$. Similar behavior was observed in the space time yield shown in Figure B.85, with the maximum $\mathrm{C}_{2}+$-oxygenate STY of $370 \mathrm{~g} / \mathrm{L}_{\mathrm{cat}} / \mathrm{hr}$ occurring at $300^{\circ} \mathrm{C}$ and $11,000 \mathrm{~L} / \mathrm{L}_{\text {cat }} / \mathrm{hr}$, before deactivation occurred. Carbon selectivity to $\mathrm{C}_{2}+$ oxygenates decreased with increasing temperature, and did not appear to be affected by the space velocity as shown in Figure B.86. However, the repeat condition for the test suggests some increase in the selectivity to $\mathrm{C}_{2}+$ oxygenates following deactivation. The opposite behavior occurred with respect to the selectivity of all oxygenates to $\mathrm{C}_{2}+$ alcohols, as shown in Figure B.87 where selectivity increased with increasing temperature, as expected, but the repeat condition had lower selectivity. The V-promoted catalyst also produced methanol, but the trends were not clear. Carbon selectivities oxygenates to methanol were approximately $2,3,5,13$, and $20 \%$, for the first five conditions tested, and approximately $1 \%$ for the repeat condition at $275^{\circ} \mathrm{C}$ and $750 \mathrm{~L} / \mathrm{L}_{\text {cat }} / \mathrm{hr}$ space velocity. Figure B.88 shows that the production of hydrocarbon liquids did not occur at temperatures above $300^{\circ} \mathrm{C}$ and that the production of higher hydrocarbon gases and liquids were much lower for the repeat condition at $275^{\circ} \mathrm{C}$.

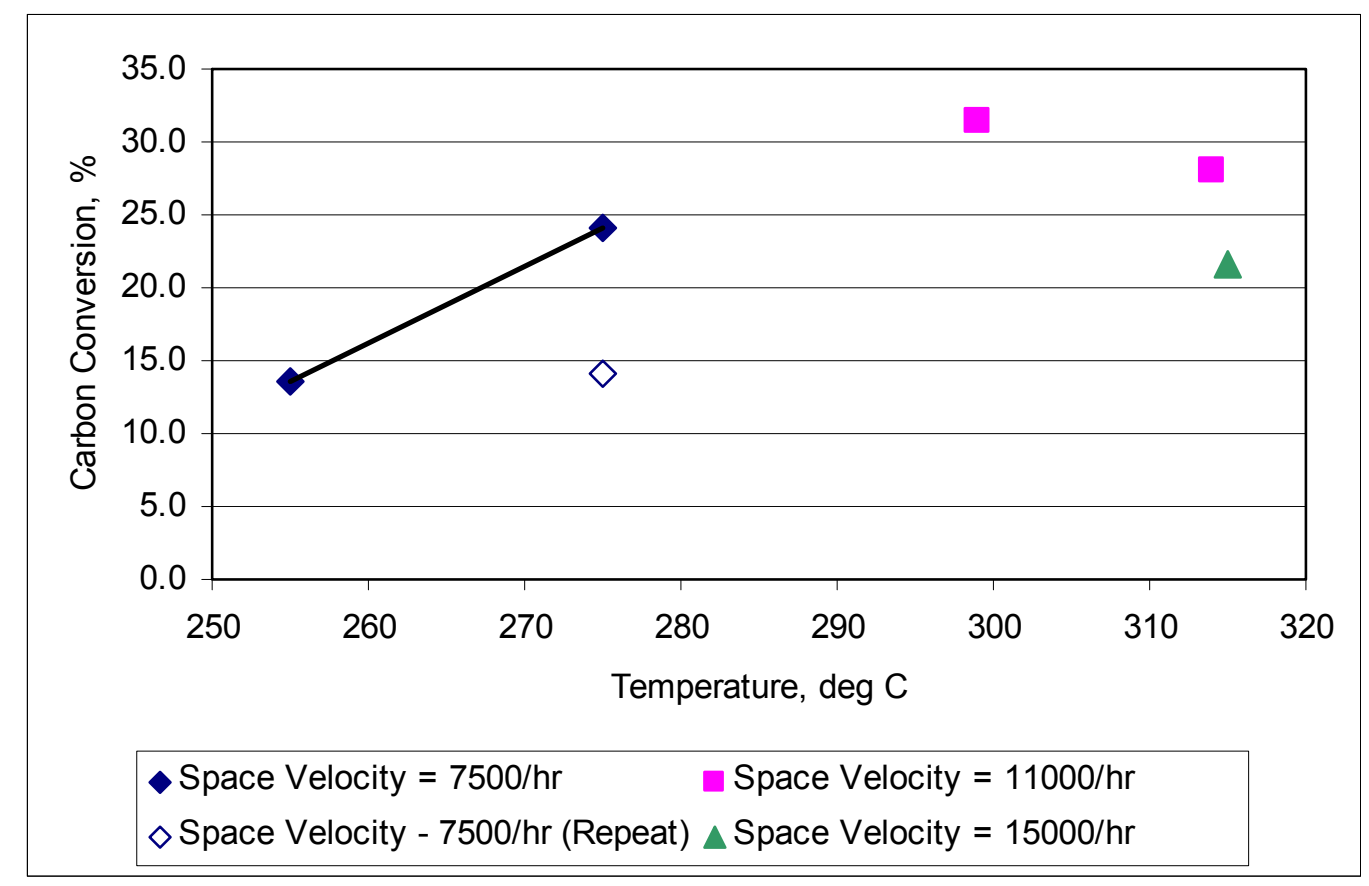

Figure B.84. Carbon Conversion for the V-Promoted Catalyst 


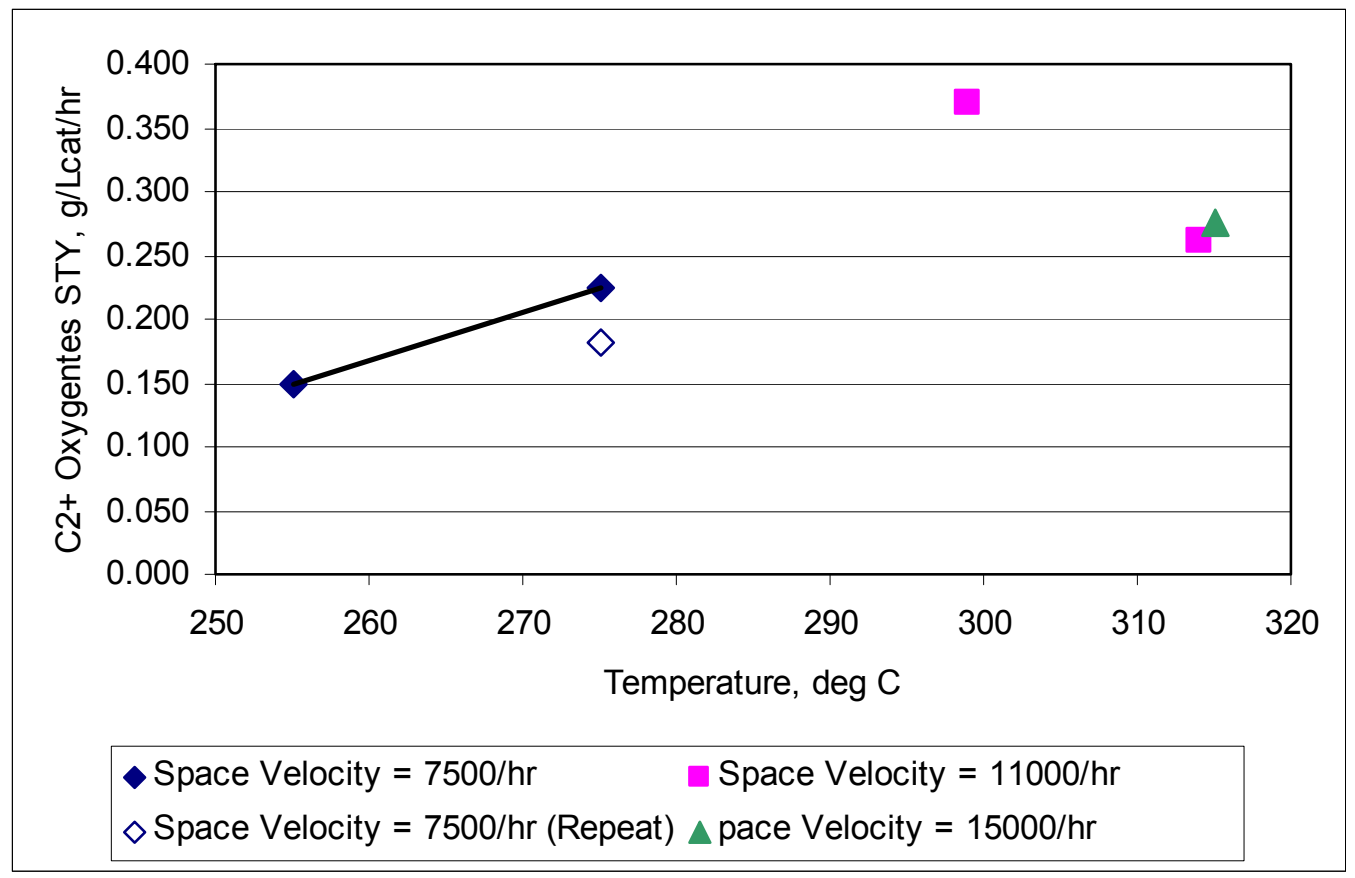

Figure B.85. $\mathrm{C}_{2}+-$ Oxygenate STYs for the V-Promoted Catalyst

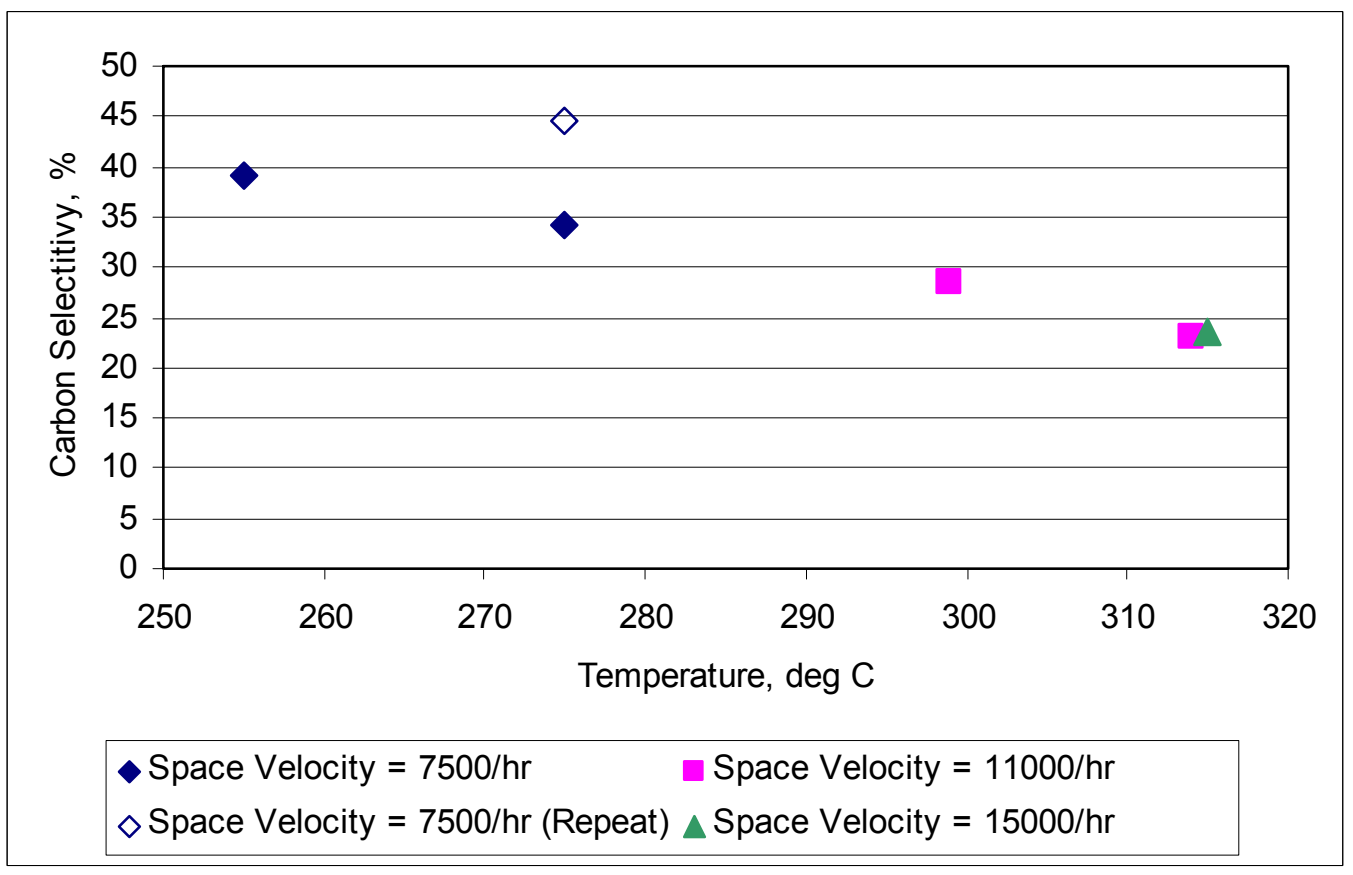

Figure B.86. Converted Carbon Selectivity to $\mathrm{C}_{2}+$ Oxygenates for the V-Promoted Catalyst 


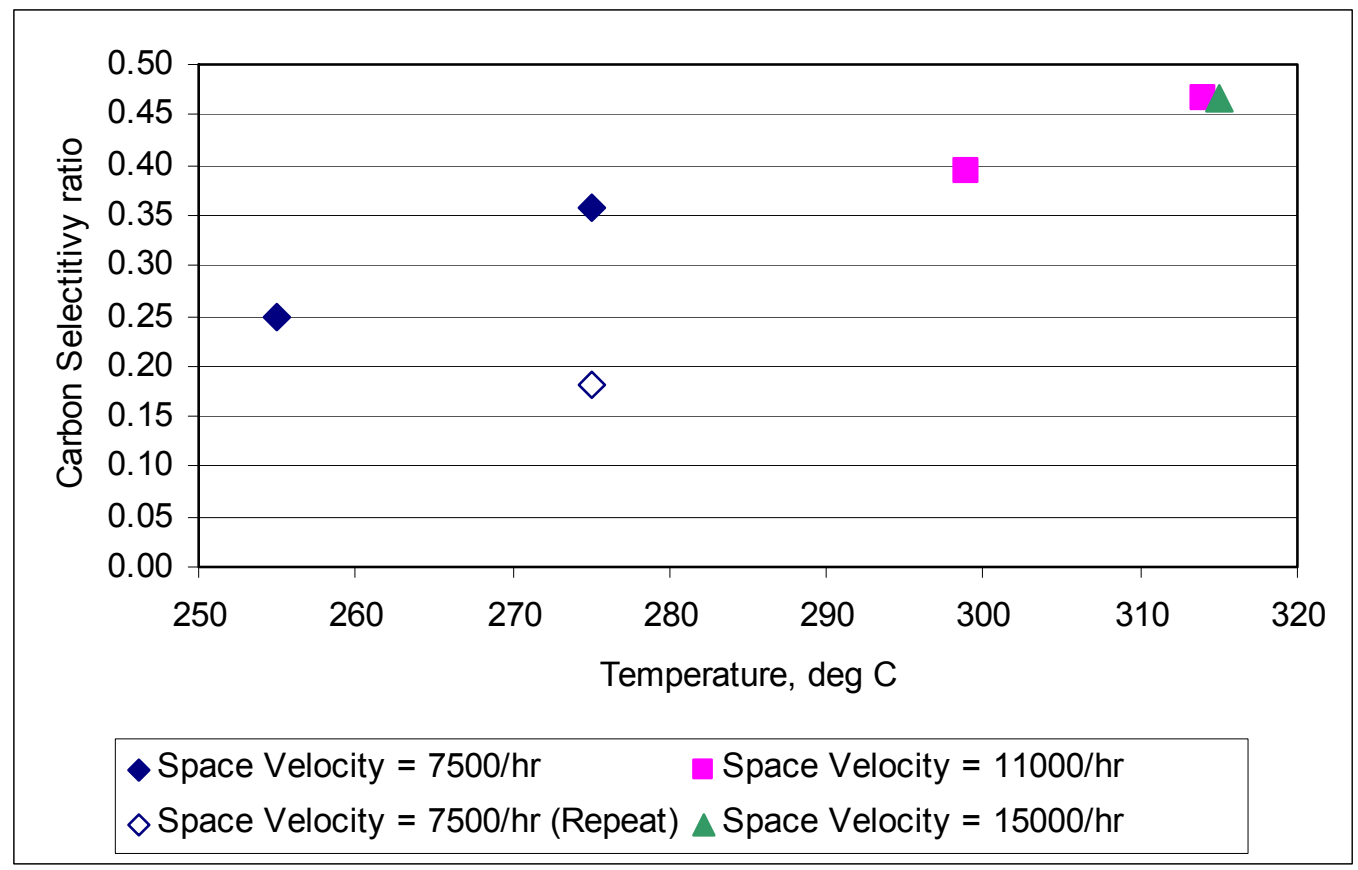

Figure B.87. Carbon Selectivity of All Oxygenates to $\mathrm{C}_{2}+$ Alcohols for the V-Promoted Catalyst

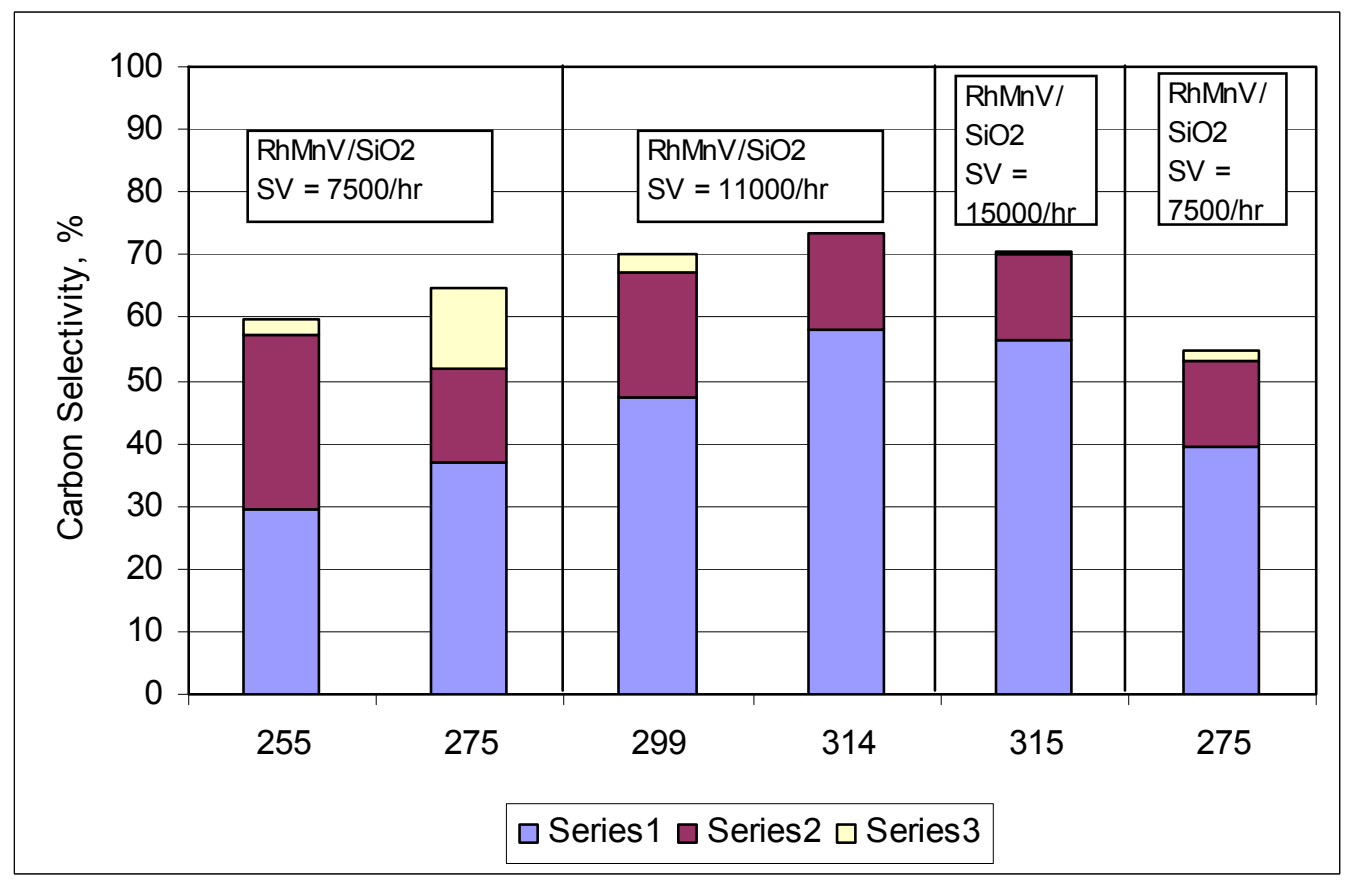

Figure B.88. Comparison of Hydrocarbon Selectivity for the V-Promoted Catalyst 


\section{B.18 Cs-Promoted Catalyst}

The Cs-promoted catalyst $\left(\mathrm{RhMnCs} / \mathrm{SiO}_{2}\right)$ catalyst was reduced at the maximum temperature of $350^{\circ} \mathrm{C}$ and tested using the hot oil circulating system to heat the reactor. The catalyst testing sequence was 257 and $276^{\circ} \mathrm{C}$ at $7500 \mathrm{~L} / \mathrm{L}_{\text {cat }} / \mathrm{hr}$, and 301 and $325^{\circ} \mathrm{C}$ at $11,000 \mathrm{~L} / \mathrm{L}_{\text {cat }} / \mathrm{hr}$. The temperature control was very good for all test conditions, with the catalyst temperature varying $\pm 1.0^{\circ} \mathrm{C}$ for the test conditions.

Figure B.89 shows that the carbon conversion was relatively low for the Cs-promoted catalyst reaching a maximum carbon conversion of $11 \%$ at $325^{\circ} \mathrm{C}$ and $11,000 \mathrm{~L} / \mathrm{L}_{\text {cat }} / \mathrm{hr}$. Carbon conversion did not appear to be affected by the space velocity at $301^{\circ} \mathrm{C}$ but appeared to experience a significant improvement at $325^{\circ} \mathrm{C}$, suggesting that the catalyst was more active than expected at the higher temperature. The $\mathrm{C}_{2}+$-oxygenate STYs were also relatively modest reaching a maximum of $116 \mathrm{~g} / \mathrm{L}_{\text {cat }} / \mathrm{hr}$ at $325^{\circ} \mathrm{C}$ as shown in Figure B.90. Carbon selectivity to $\mathrm{C}_{2}+$ oxygenates initially increased between 257 and $276^{\circ} \mathrm{C}$ as shown in Figure B.91, but then decreased at the higher temperatures. According to Figure B.92, carbon selectivity of all oxygenates to $\mathrm{C}_{2}+$ alcohols was low, with a maximum selectivity of approximately $24 \%$ at $257^{\circ} \mathrm{C}$ and then decreasing to between approximately 10 and $11 \%$ at the higher temperatures. Figure B.93 shows that hydrocarbon liquids were not produced by the Cs-promoted catalyst and that the carbon selectivity to higher hydrocarbon gases initially decreased from $33 \%$ at $257^{\circ} \mathrm{C}$ to a relatively constant 18 to $22 \%$ at the higher temperatures.

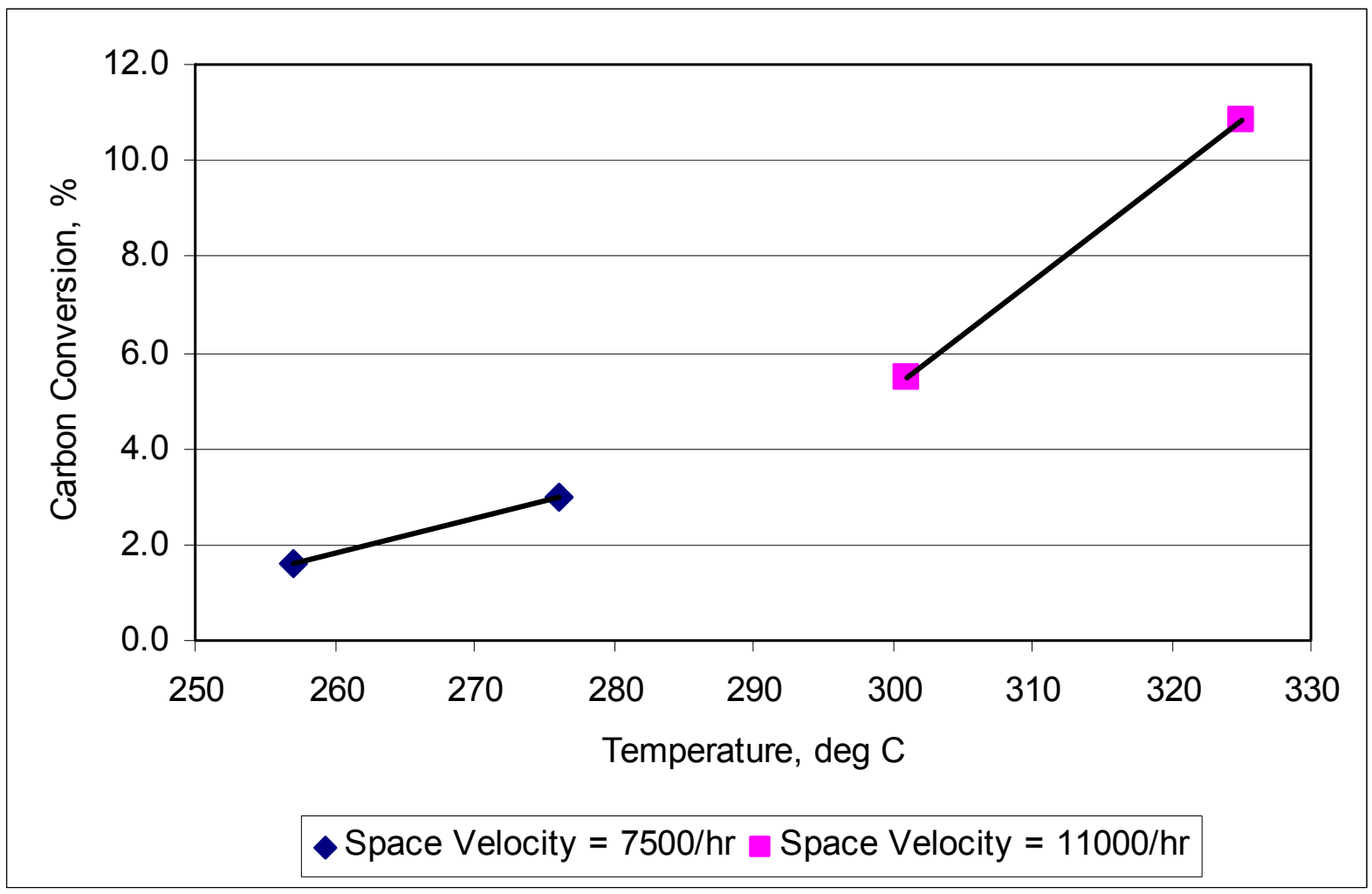

Figure B.89. Carbon Conversion for the Cs-Promoted Catalyst 


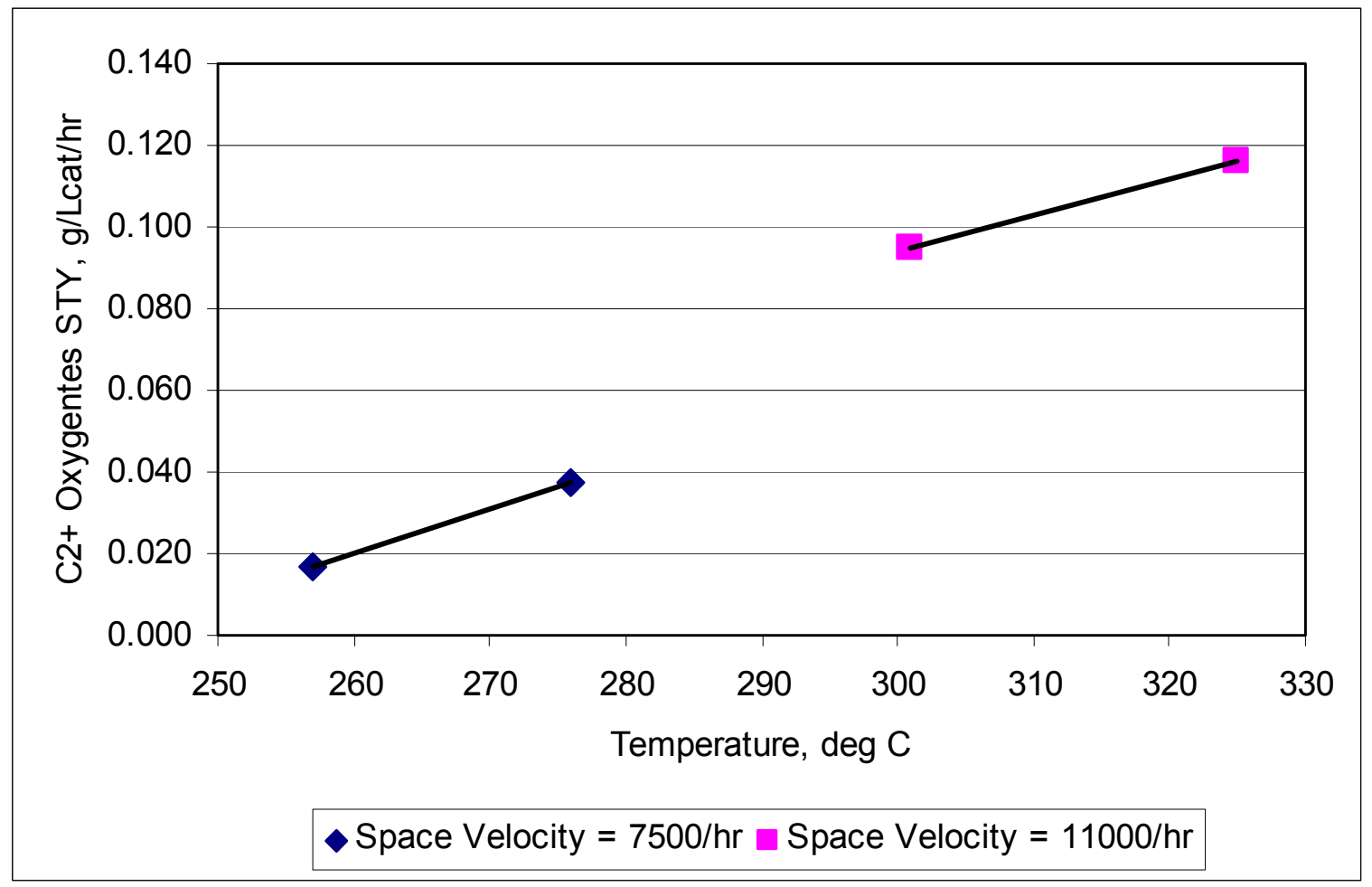

Figure B.90. $\mathrm{C}_{2}+-$ Oxygenate STYs for the Cs-Promoted Catalyst

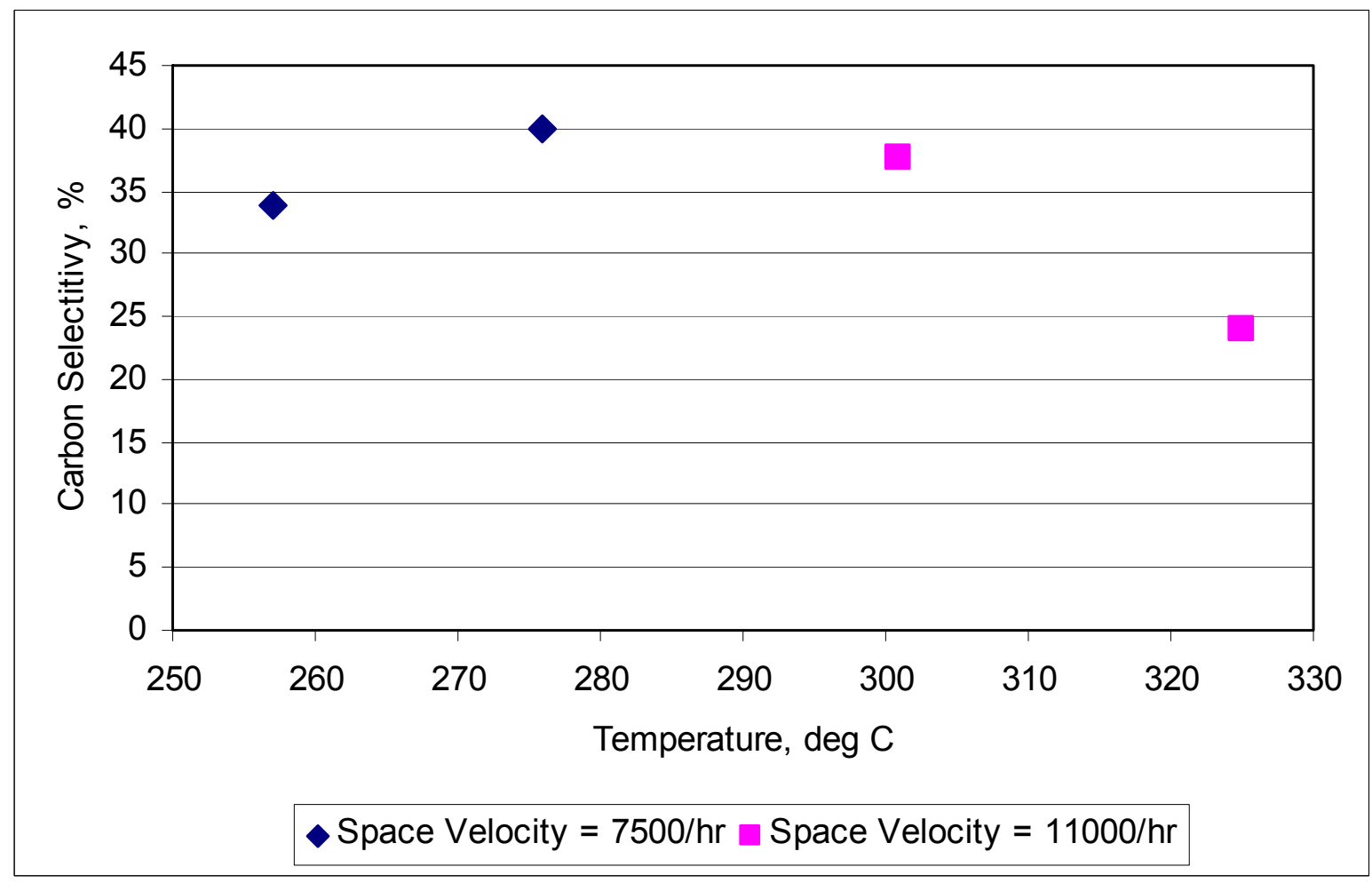

Figure B.91. Converted Carbon Selectivity to $\mathrm{C}_{2}+$ Oxygenates for the Cs-Promoted Catalyst 


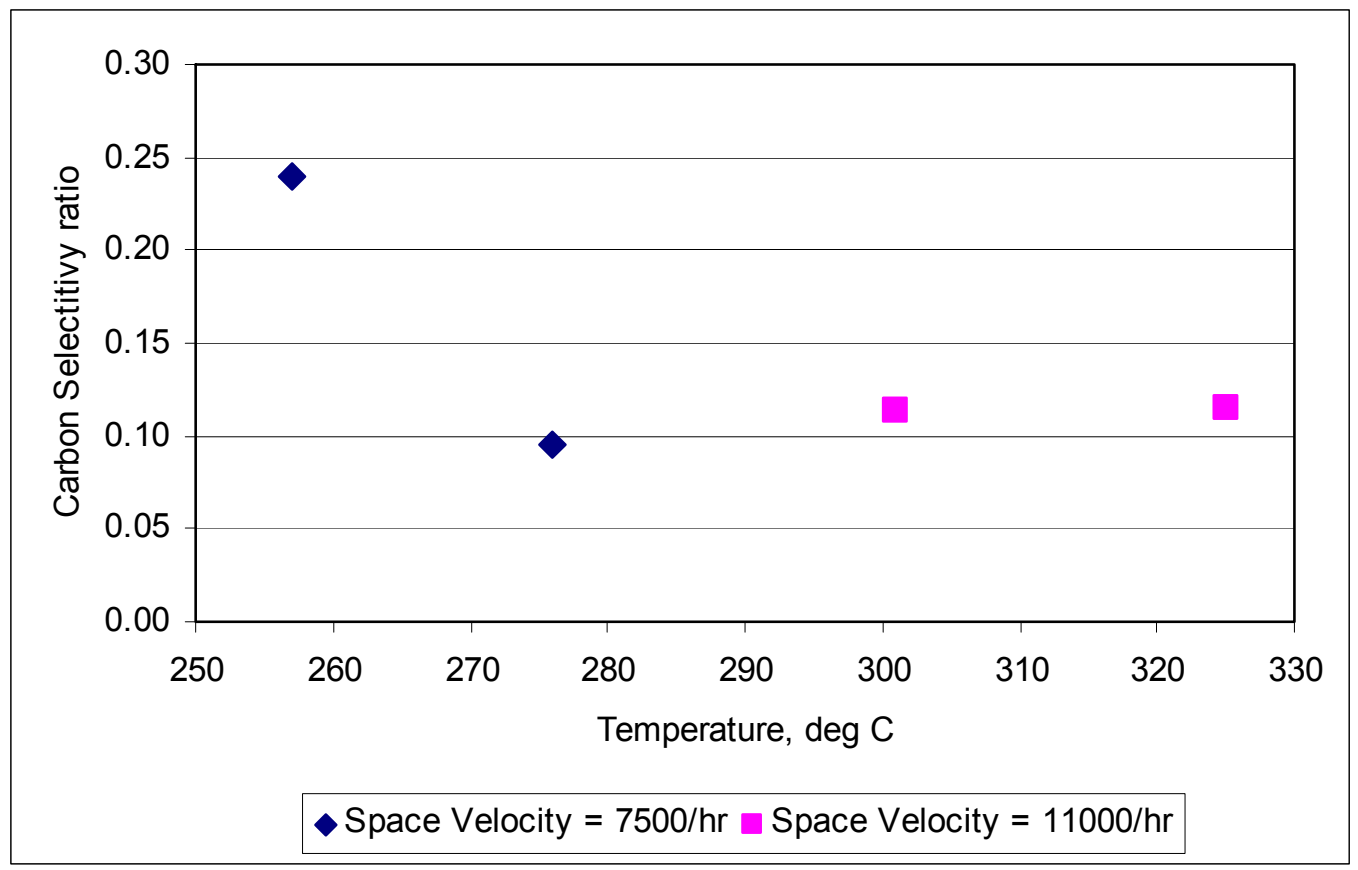

Figure B.92. Carbon Selectivity of All Oxygenates to $\mathrm{C}_{2}+$ Alcohols for the Cs-Promoted Catalyst

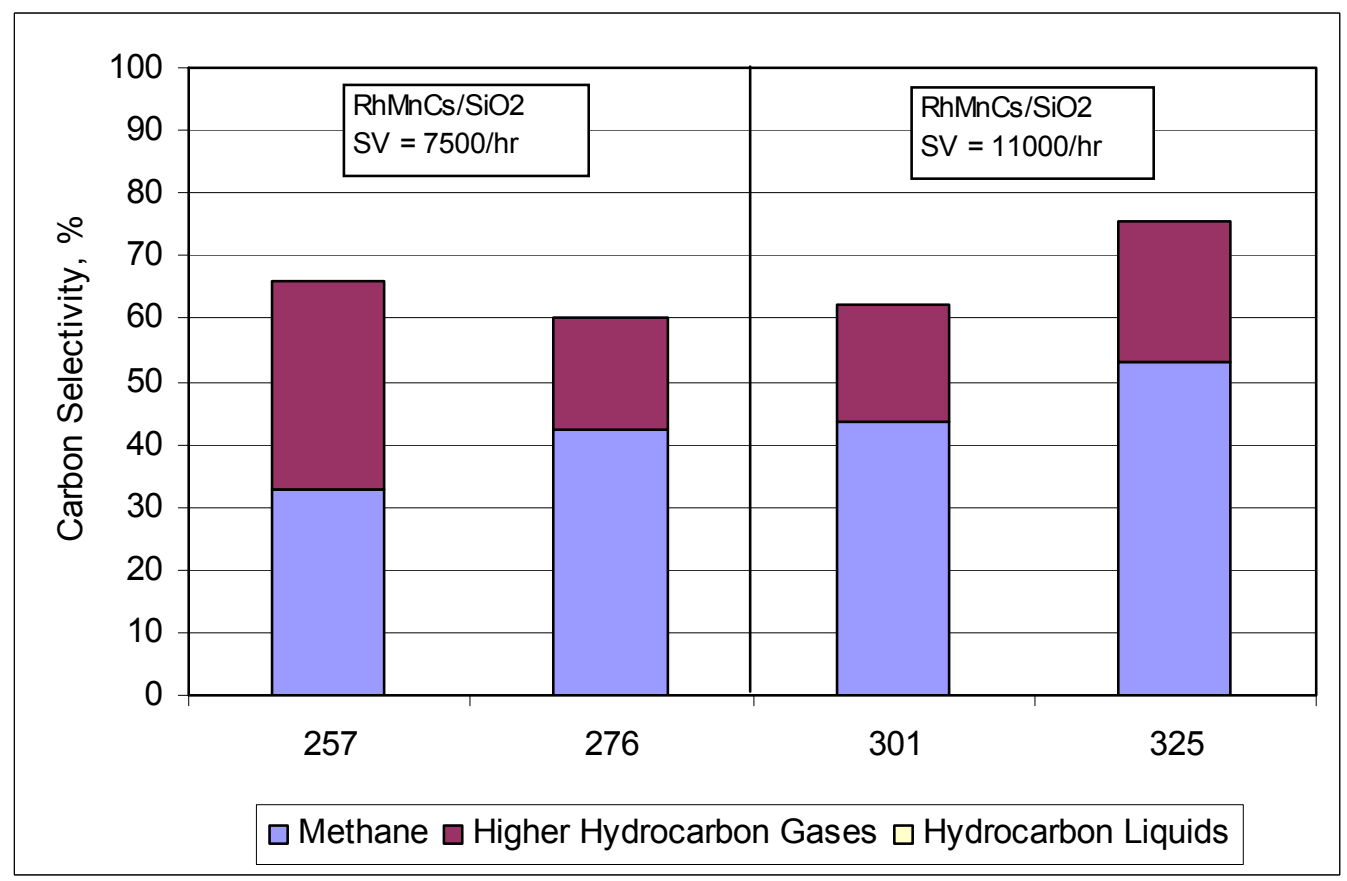

Figure B.93. Comparison of Hydrocarbon Selectivity for the Cs-Promoted Catalyst 


\section{B.19 Catalysts Promoted With $\mathrm{Ge}, \mathrm{Te}, \mathrm{Pb}$, or $\mathrm{Bi}$}

$\mathrm{RhMn} / \mathrm{SiO}_{2}$ catalysts promoted with $\mathrm{Ge}, \mathrm{Te}, \mathrm{Pb}$, or $\mathrm{Bi}$ were reduced at the maximum temperature of $350^{\circ} \mathrm{C}$ and tested using the hot oil circulating system to heat the reactor. The catalyst testing sequence was 257 and $276^{\circ} \mathrm{C}$ at 7500 at $\mathrm{L} / \mathrm{L}_{\text {cat }} / \mathrm{hr}$, and 301 and $325^{\circ} \mathrm{C}$ at $11,000 \mathrm{~L} / \mathrm{L}_{\text {cat }} / \mathrm{hr}$ for each catalyst. The Bi-promoted catalyst also was tested at $345^{\circ} \mathrm{C}$ and $11,000 \mathrm{~L} / \mathrm{L}_{\text {cat }} / \mathrm{hr}$. All of the catalysts were inactive, achieving no more that $1.5 \%$ carbon conversion at the highest temperature tested. The Te-promoted catalyst showed no activity at all, while the Ge-promoted catalyst produced very small quantities of liquid product at all temperatures, of which $95 \%$ of the organics in the liquid were $\mathrm{C}_{1}+$ alcohols. The $\mathrm{Pb}$ - and Bi-promoted catalysts produced very small quantities of liquids at the two highest temperatures tested, of which approximately $70 \%$ were $\mathrm{C}_{1}+$ alcohols. Methane was the only hydrocarbon produced by the $\mathrm{Ge}-, \mathrm{Pb}-$, and Bi-promoted catalysts. 




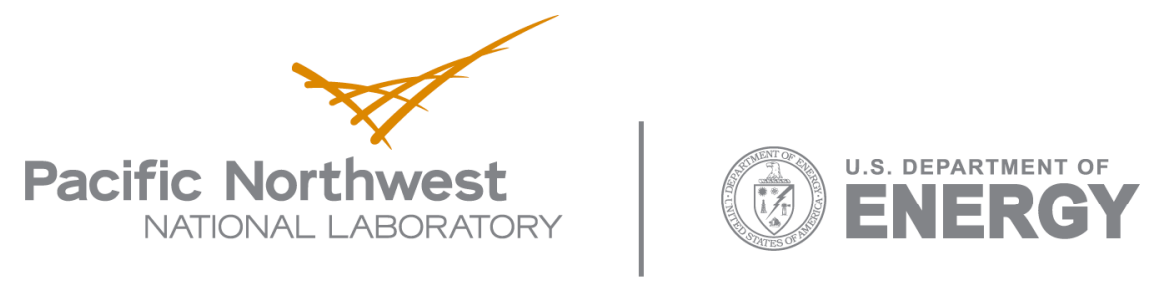

902 Battelle Boulevard

P.O. Box 999

Richland, WA 99352

1-888-375-PNNL (7665)

www.pnl.gov 